Florida International University FIU Digital Commons

4-12-2010

\title{
Development of distributed generation infrastructure for microgrid connectivity to operational power systems
}

Nayeem Mohammad Abdullah

Florida International University

DOI: $10.25148 /$ etd.FI13101507

Follow this and additional works at: https://digitalcommons.fiu.edu/etd

Part of the Electrical and Computer Engineering Commons

\section{Recommended Citation}

Abdullah, Nayeem Mohammad, "Development of distributed generation infrastructure for microgrid connectivity to operational power systems" (2010). FIU Electronic Theses and Dissertations. 1099.

https://digitalcommons.fiu.edu/etd/1099 
FLORIDA INTERNATIONAL UNIVERSITY

Miami, Florida

DEVELOPMENT OF DISTRIBUTED GENERATION INFRASTRUCTURE FOR MICROGRID CONNECTIVITY TO OPERATIONAL POWER SYSTEMS

A thesis submitted in partial fulfillment of the

requirements for the degree of

MASTER OF SCIENCE

in

ELECTRICAL ENGINEERING

by

Nayeem Mohammad Abdullah 
To: Dean Amir Mirmiran

College of Engineering and Computing

This thesis, written by Nayeem Mohammad Abdullah, and entitled Development of Distributed Generation Infrastructure for Microgrid Connectivity to Operational Power Systems, having been approved in respect to style and intellectual content, is referred to you for judgment.

We have read this thesis and recommend that it be approved.

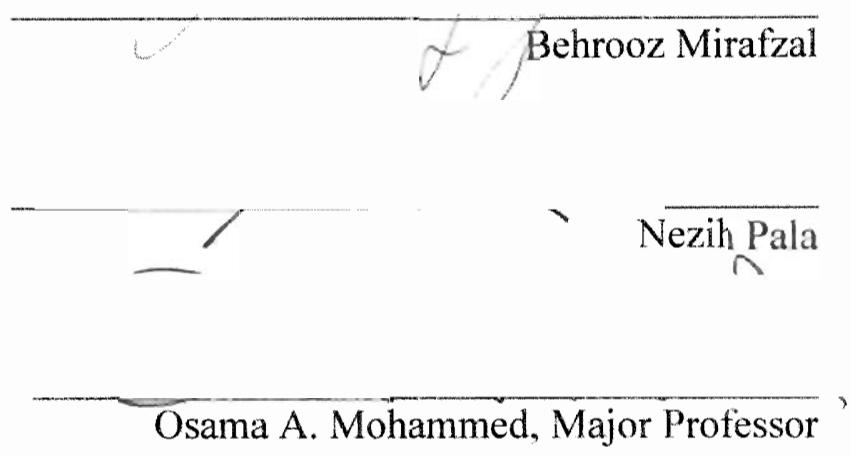

Date of Defense: April 12, 2010

The thesis of Nayeem Mohammad Abdullah is approved.

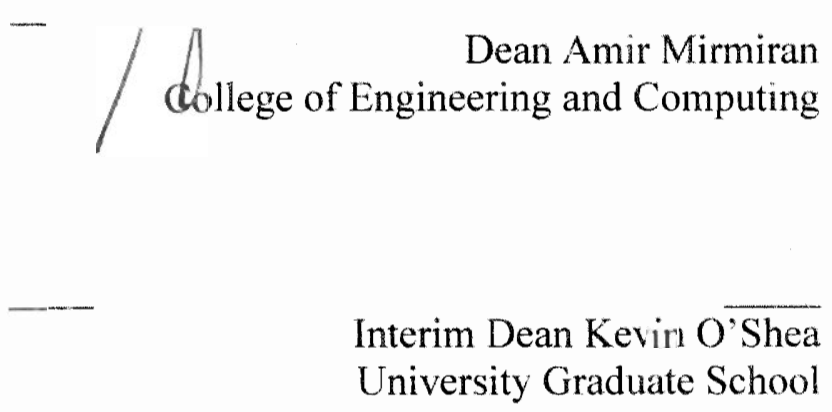

Florida International University. 2010 


\section{DEDICATION}

I dedicate this work to my dear parents Janab Faheem Sahab and Mohtarma Haleema Begum for their love, support, encouragement and everything else they have given me so far in life. Also, I express deepest gratitude and dedication towards my brothers Azeem, Raheem and Waseem for instilling unfettered faith in me, and enduring my responsibilities towards my parents and straining themselves with all the family work during my course of study. I would also like to express my gratitude for unrelenting and unconditional moral support of my friends Aijaz, Sandeep, Siddiq and Ali. 


\section{ACKNOWLEDGMENTS}

I would like to express my deepest gratitude and special thanks to my graduate advisor, Professor Osama A. Mohammed for providing me this opportunity to work on this funded research project.

This work is based on Dr. Mohammed's idea of creating a distributed generation, microgrid interconnection power system test bed that would emulate on shipboard and airplane power systems along with modern electric utility power system. This work represents a portion of this work and I would like to gratefully acknowledge his technical guidance and mentorship which made this thesis possible. I would also like to acknowledge his help and encouragement in writing this thesis. My sincere thanks are also due to my graduate committee members Dr. Behrooz Mirafzal and Dr. Nezih Pala for all the support they have given me during this work.

I would like to acknowledge and thank all my laboratory colleagues and mention Dr. A. Khan, Mr. A. Kashefi and Mr. M. Elshaer for their support. 


\begin{abstract}
OF THE THESIS
DEVELOPMENT OF DISTRIBUTED GENERATION INFRASTRUCTURE FOR MICROGRID CONNECTIVITY TO OPERATIONAL POWER SYSTEMS
\end{abstract}

by

Nayeem Mohammad Abdullah

Florida International University, 2010

Miami, Florida

Professor Osama A. Mohammed, Major Professor

Distributed Generation (DG) from alternate sources and smart grid technologies represent good solutions for the increase in energy demands. Employment of these DG assets requires solutions for the new technical challenges that are accompanied by the integration and interconnection into operational power systems.

A DG infrastructure comprised of alternate energy sources in addition to conventional sources, is developed as a test bed. The test bed is operated by synchronizing, wind, photovoltaic, fuel cell, micro generator and energy storage assets, in addition to standard AC generators. Connectivity of these DG assets is tested for viability and for their operational characteristics. The control and communication layers for dynamic operations are developed to improve the connectivity of alternates to the power system. A real time application for the operation of alternate sources in microgrids is developed. Multi agent approach is utilized to improve stability and sequences of actions for black start are implemented. Experiments for control and stability issues related to dynamic operation under load conditions have been conducted and verified. 


\section{TABLE OF CONTENTS}

1. INTRODUCTION .1

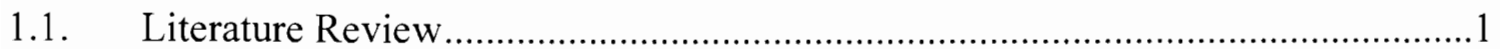

1.2. Grid Integration Challenges with Sustainable Energy Resources .........................4

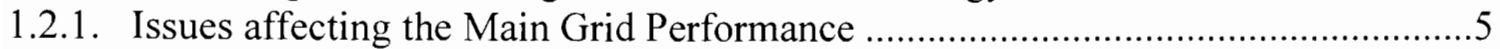

1.2.2. Issues for Microgrid Energy Sharing and Power Quality .....................................

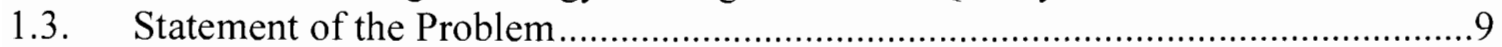

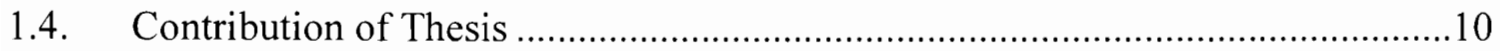

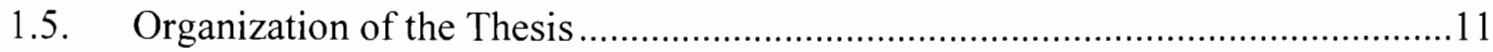

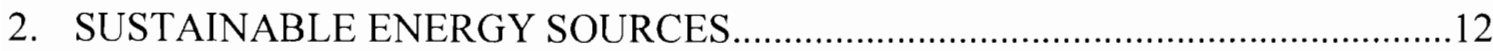

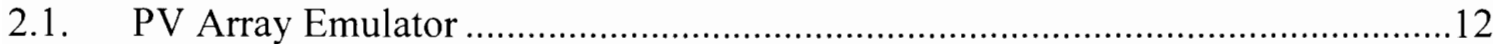

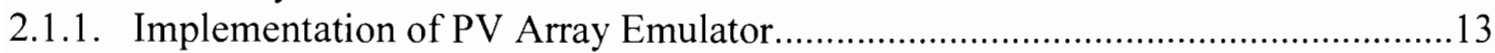

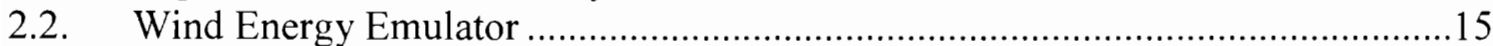

2.2.1. Model for Wind Energy Emulator ..................................................................... 15

2.2.2. Implementation of Wind Emulator on DC Machine and Induction Generator set 17

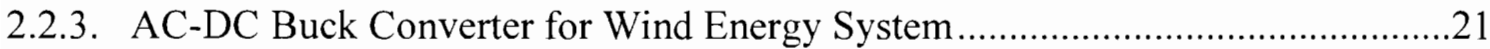

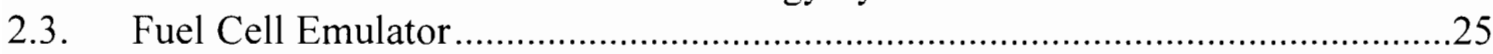

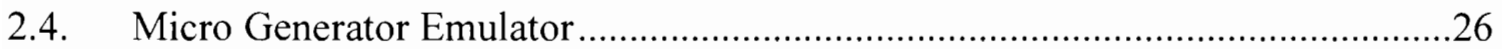

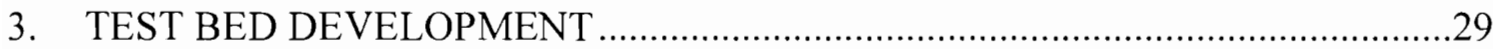

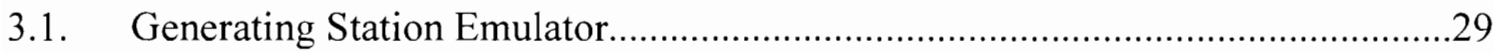

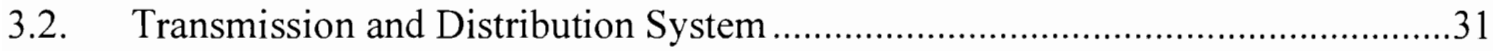

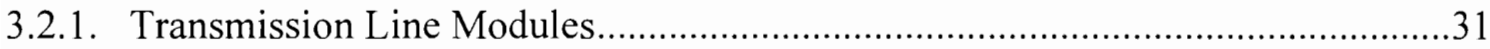

3.2.2. Transformer based High Voltage Transmission Line ...........................................33

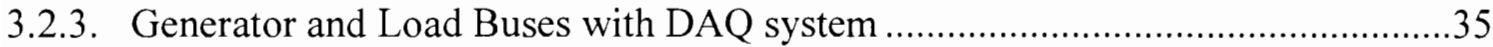

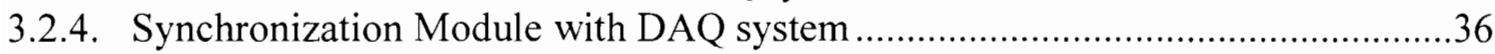

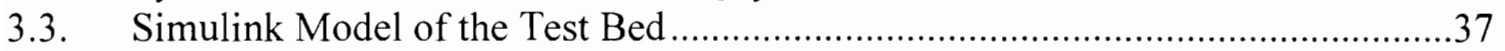

3.4. Block Diagram for Hardware Test Bed Implementation ......................................39

4. IMPLEMENTATION OF SOURCES AND LOADS ON THE TEST BED ............41

4.1. AVR Implementation on the Generating Station...............................................

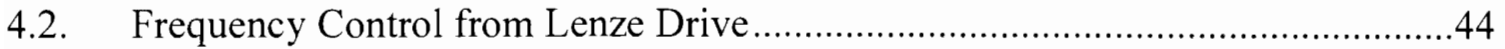

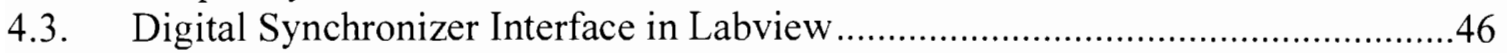

4.4. Load Connection and other System Integration..................................................

4.5. Architectures for Test Bed Configuration...........................................................50

5. IMPLEMENTING A MICROGRID EMULATOR WITH ALTERNTE SOURCE 54

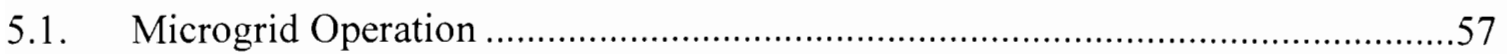

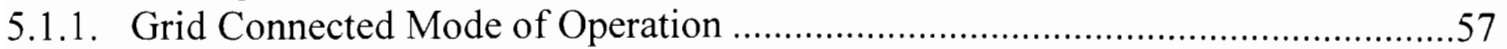

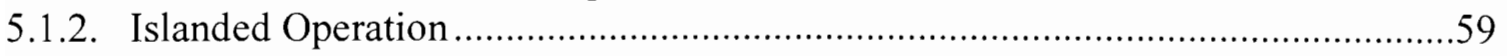




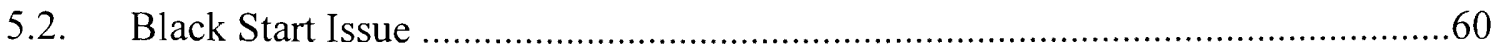

5.3. Microgrid Model Implemented on the Test Bed................................................61

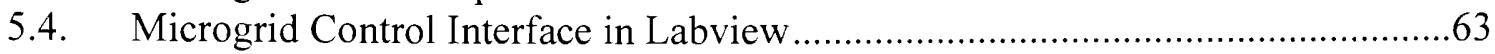

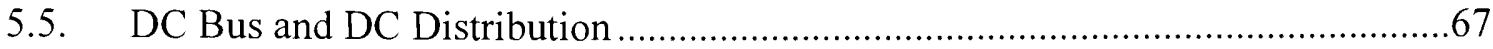

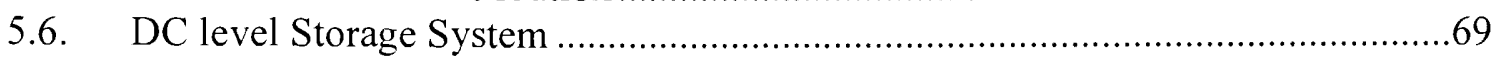

6. CONTROL AND COMMUNICATION INFRASTRUCTURE ..............................71

6.1. Control and Data Acquisition Devices used on the Test Bed ...............................71

6.2. Communication Components and Technologies Implemented on the Test Bed...72

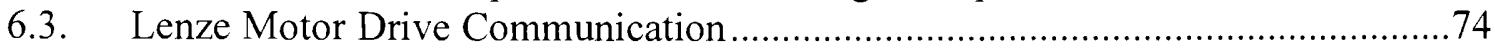

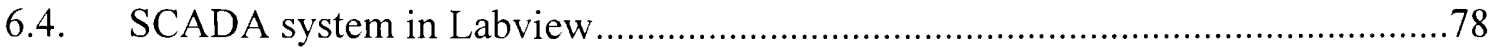

6.5. Simulation of an Agent Framework for Test Bed Application...............................82

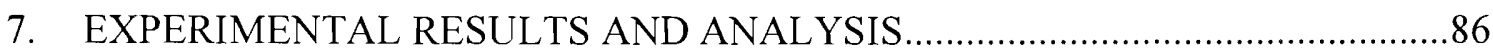

7.1. Experiment 1: Energy Sharing between Test Bed and Microgrid ..........................86

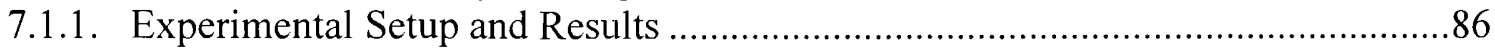

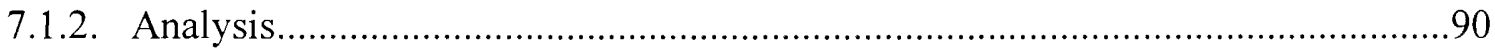

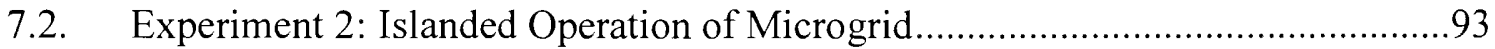

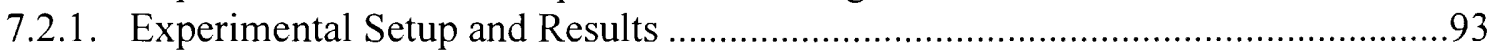

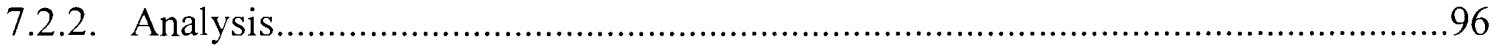

7.3. Experiment 3: Wind Energy Integration to Test Bed .......................................97

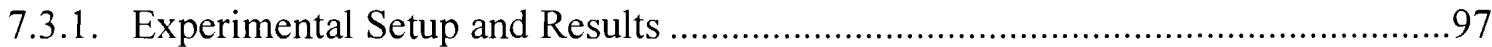

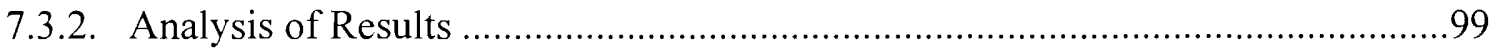

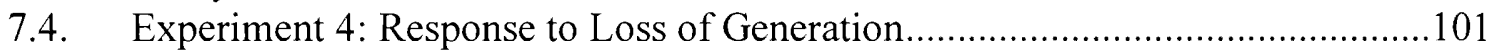

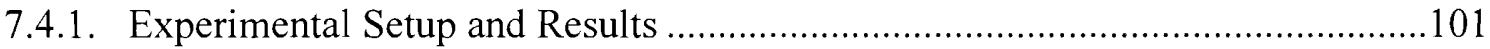

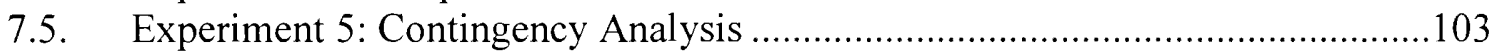

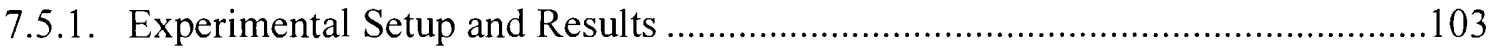

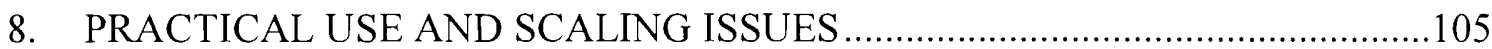

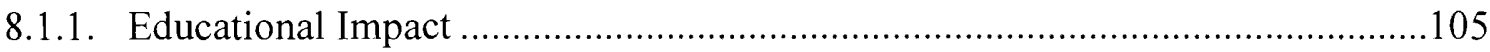

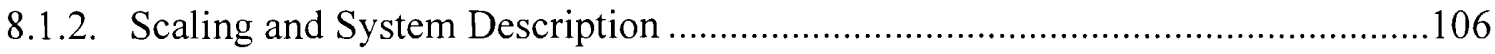

9. DISCUSSION OF OVERALL RESEARCH RESULTS .....................................107

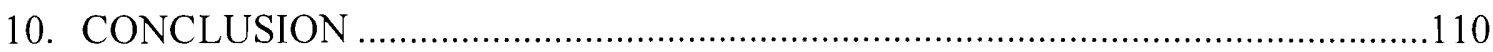

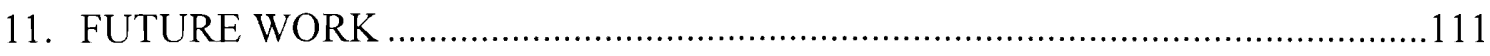

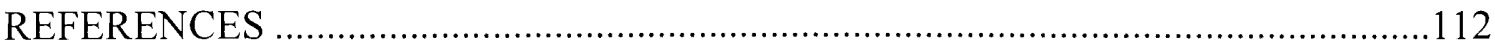

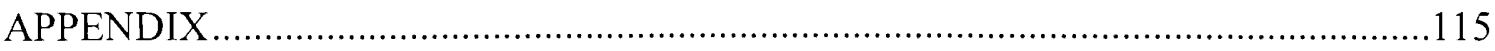




\section{LIST OF FIGURES}

\section{FIGURE}

Figure 2.1-1 Circuit Diagram representing a PV Array equivalent circuit 12

Figure 2.1-2 PV Array block diagram and interface model......................................... 13

Figure 2.1-3 Experimental set up for PV array emulator using Motor Generator set ...... 14

Figure 2.1-4 DC Voltage output of the DC generator and AC output of the GTI ............ 15

Figure 2.2-1 Wind Energy Emulator Block Diagram .................................................... 16

Figure 2.2-2 Real time Simulink model for wind turbine emulator on a DC motor......... 17

Figure 2.2-3 Power electronic drives for Speed variation ........................................... 18

Figure 2.2-4 Capacitor based field excitation module for Induction generator ................ 19

Figure 2.2-5 Hampden Induction Generator ............................................................. 20

Figure 2.2-6 Motor solver Induction Generator......................................................... 20

Figure 2.2-7 Start up characteristics of Induction Generator....................................... 21

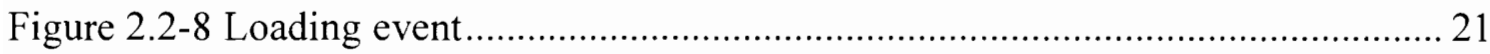

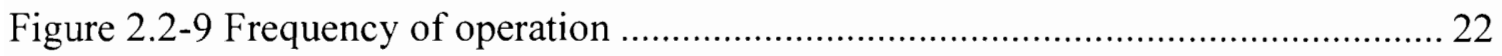

Figure 2.2-10 Rectifier module Simulink model and DC voltage output waveform........ 23

Figure 2.2-11 Rectifier module with L-C filter ........................................................ 24

Figure 2.2-12 dSPACE/Simulink Buck Converter module for Wind Energy Generator . 25

Figure 2.2-13 Semikron AC/DC Buck Converter for Wind Generator ............................ 25

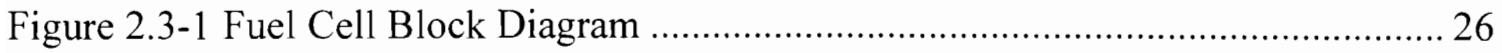

Figure 2.3-2 Fuel Cell Emulator from DC Power Supply ........................................... 26

Figure 2.4-1 Simulink Model for Combustion Generator Emulator Control .................. 28

Figure 2.4-2 Combustion Generator User Interface …............................................ 29 
Figure 2.4-3 Power Electronic Drive Boards for DC Motor and Alternator field control 29

Figure 3.1-2 Generating station front view

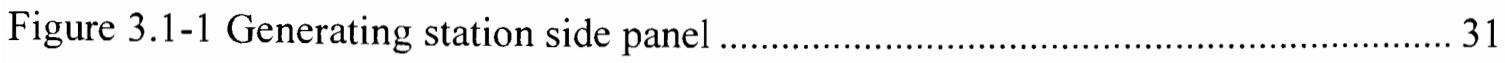

Figure 3.2-1 Simulink model of the partial test bed model with transformers line .......... 35

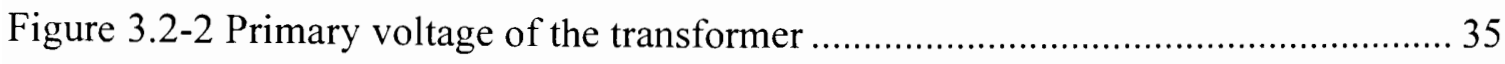

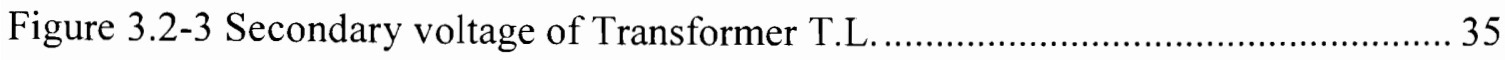

Figure 3.2-4 Hardware Implementation of Transformers based line.............................. 36

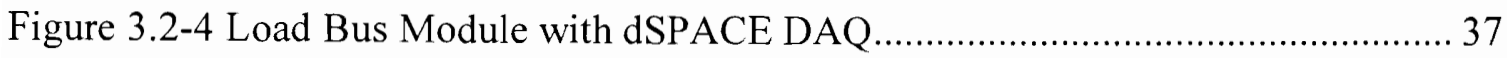

Figure 3.2-5 Synchronizer module with Labview RTU Connector................................ 38

Figure 3.3-1 Simulink model of the power system test bed and microgrid platform ....... 40

Figure 3.3-2 Simulink model for a load center with generation emulating a microgrid .. 41

Figure 3.4-1 Block diagram representation of the power system test and microgrid....... 42

Figure 4.1-1 AVR block diagram with the excitation field and electronic interface........ 44

Figure 4.1-2 AVR installed on the front panel of the alternator ..................................... 44

Figure 4.1-3 Experimental setup for AVR and frequency response of the alternator ...... 45

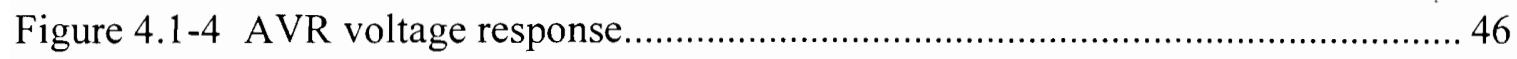

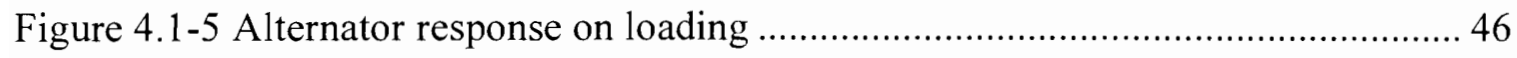

Figure 4.3-1 Synchronizing Module operation ........................................................ 49

Figure 4.3-2 Synchronizing Module, Labview V.I. Block Diagram and Front Panel...... 50

Figure 4.5-1 Two Tier Zonal Architecture connection of the test bed ............................ 53

Figure 4.5-2 Ring Bus Architecture of the test bed .................................................. 53

Figure 4.5-3 Serial Architecture with Three generator Test bed ................................... 54

Figure 4.5-4Zonal Architecture with Distributed Generation...................................... 54 
Figure 4.5-5 Hardware Implementation of Test Bed. 55

Figure 5.1-1 Microgrid Synchronized Power Consumption Mode 60

Figure 5.1-2 Microgrid Synchronized Power Injection Mode. 61

Figure 5.1-3 Microgrid Islanded Mode of Operation 62

Figure 5.3-1 Block Diagram of the Microgrid Model Implemented on the Test Bed 64

Figure 5.3-2 Hardware Implementation of Microgrid with DC Bus 65

Figure 5.4-1 Labview VI program for Microgrid Control and Monitoring 67

Figure 5.5-1 Block Diagram of DC Distribution system within the AC test bed. 70

Figure 5.5-2 DC Distribution system on the Test Bed.................................................. 71

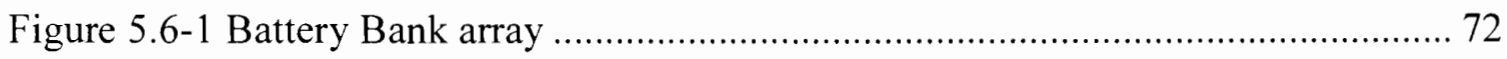

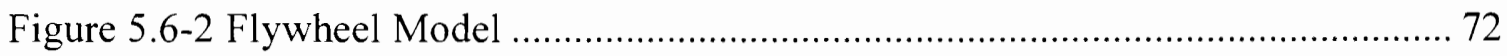

Figure 6.1-1 NI 6259M BNC Test Bed AC system Control Board ................................. 74

Figure 6.2-1 NI 6259USB DAQ system used for Microgrid Interface VI in chapter 5.375

Figure 6.3-1 Drive Terminal Hardware Connection..................................................... 76

Figure 6.3-2 Network Control of Lenze Motor Drive ……........................................... 77

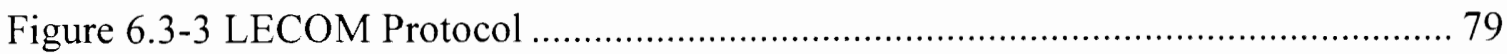

Figure 6.3-4 Block Diagram for Data Acquiring VI for LECOM protocol...................... 80

Figure 6.4-1 User Interface of SCADA system of the Test Bed .................................. 82

Figure 6.4-2 Synchronizer Module VI published on the Internet ................................. 83

Figure 6.5-1 Multi Agent System application in power systems.................................. 85

Figure 6.5-2 Multi Agent System application in power systems .................................... 86

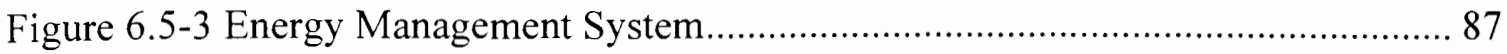

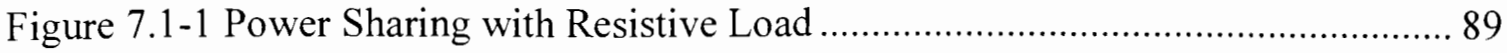


Figure 7.1-2 Generating Station Catering to the R-Load.

Figure 7.1-3 Steady State Response of Gen connected to R-Load

Figure 7.1-4 Gen and GTI sharing R-Load

Figure 7.1-5 GTI and Gen Sharing R-Load

Figure 7.1-6 Load Bus and DC Bus

Figure 7.1-7 Gen V and I RMS (Single Phase)

Figure 7.1-8 GTI V and I RMS (Single Phase)

Figure 7.1-9 Load Bus V and I (Single Phase)

Figure 7.1-10 DC Bus V and I (Three Phase Input to GTI's).

Figure 7.1-11 Power Values at different Buses

Figure 7.2-1 Islanded Operation Experiment

Figure 7.3-1 Setup for Wind Energy injection into test bed

Figure 7.3-2 Wind Gen Voltage.

Figure 7.3-3 Wind Gen Voltage and Current at GTI Load

Figure 7.3-4 Wind Gen V/I and rectified DC Voltage (GTI I/P)

Figure 7.3-5 GTI V/I with Wind Input

Figure 7.3-6 Load Bus Response on Wind Source Input

Figure 7.4-1 Loss of Generation

Figure 7.5-1 Frequency Response for self clearing fault 


\section{LIST OF ACRONYMS}

Acronym

Full Form

AMS Agent Management System

AVR Automatic Voltage Regulator

API Application Programming Interface

DAQ Data Acquisition

DF Directory Facilitator

DG Distributed Generation

DSP Digital Signal Processor

FIPA Foundation for Intelligent Physical Agents

GTI Grid Tie Inverter

GUI Graphic User Interface

IDE Integrated Development Environment

IPP Independent Power Producer

JADE Java Agent Development Framework

JRE Java Runtime Environment

LV Low Voltage

MAS Multi Agent System

MG Micro grid

MPPT Maximum Power Point Tracking

NERC North American Reliability Council

PCC Point of Common Coupling 


\section{LIST OF ACRONYMS}

Acronym

Full Form

AMS

Agent Management System

AVR

Automatic Voltage Regulator

API

Application Programming Interface

DAQ

Data Acquisition

DF

Directory Facilitator

DG

Distributed Generation

DSP

Digital Signal Processor

FIPA

Foundation for Intelligent Physical Agents

GTI

Grid Tie Inverter

GUI

Graphic User Interface

IDE

Integrated Development Environment

IPP

Independent Power Producer

JADE

Java Agent Development Framework

JRE

Java Runtime Environment

LV

Low Voltage

MAS

Multi Agent System

MG

Micro grid

MPPT

Maximum Power Point Tracking

NERC

North American Reliability Council

PCC

Point of Common Coupling 
PV Array Photo Voltaic Array

RTU Remote Terminal Unit

SBPS Ship Board Power System

SCADA Supervisory Control and Data Acquisition System

UART Universal Asynchronous Receiver and Transmitter

UFRO Under Frequency Roll Off point

VI

Virtual Instrument 


\section{INTRODUCTION}

\subsection{Literature Review}

Technological advances have led to a greater to utilization of power frol renewable sources. The recent improvements in the power electronics field and switchin devices are enabling us to control power at greater levels with a faster response.

The increase in energy demands, coupled with constraints on investments i conventional power generation plants and ecological issues have led to increased calls $\mathrm{fc}$ the integration of non-conventional energy sources in the existing power system. $\mathrm{Tr}$ integration of non-conventional sources can be in many forms such as clusters ( generators as micro-grids, collection of microgrids as smart grids, distributed generato: and embedded generating stations. Most of these sources are installed and located at loc centers, with a primary intention to meet the local demand for uninterruptible, clean an cheap energy with increased reliability. These sources are synchronized with the mai grid so as to draw power from it in case of shortage and inject power into it during a excess generation period $[1-3]$.

All these factors promise a great future for the power system and for th consumer, but these technologies can add as many challenges as they may try to solv [4]. The present power system is a complex network of many interconnected systen operating in various geographical locations, virtually presenting a synchronize mechanical system. The non-conventional sources are mainly power electronic devis based systems with a much lower response time, posing serious synchronization issue 
The geographical distribution of these micro sources also presents a lot of co-ordinatior and control issues. The major branches of the power system, generation, transmission distribution, control, optimization and tariff, need to be reassessed in light of distributec and micro, non-conventional sources.

A lot of research efforts are directed towards improving the technology of the non-conventional sources, distributed generation and microgrids to enable smootl integration to the power system. Most of the work is based on simulation and in cases o hardware implementation, only a certain component is verified. It is an important concerr to verify the results in response to a control action of a component or a system as a resul of applying a procedure to connect distributed resource to the power system to verify the effects of integration.

The increase in the demands for energy is pushing the present generation anc transmission systems to their limits. In addition, the cost of increasing the capacity of the conventional power plants is very high and, is concentrated on one company o organization. The ecological problems caused by pollution due to the conventiona sources and pollution from coal and oil based fuel is also forcing the governments o various countries to look for alternative options.

Adding to all this, the losses suffered by power companies during black outs an brown outs are very high and render normal life services difficult where everythin! depends on electricity. This has led to the concept of distribution in generation center and also a call for independent generation. Although deregulation in developed countrie has helped in competition among the power companies benefitting the consumers, ther 
is still ground to be covered to provide continuous and independent energy. This has led the consumer to start utilizing micro sources, which provide a cheap and clean energy option at small cost in the long run.

All these factors are driving the present power systems towards integration of non-conventional micro-sources of energy. These sources will be distributed among the load centers and in wide areas geographically, with comparatively small amount of generation capability. The present centralized control architecture of the power system will have to give way towards distributed and decentralized control architecture, with independent agents making the decisions based on the persisting conditions. This will reduce any manual errors and also the time for decision making. This improvement in control architecture will suffice to provide continuous energy supply for consumers even during faults on the main grid. In addition, it will also reduce the errors which were responsible for black outs. The examples for these are the 2001 and 2003 black outs in New York and 2008 brown out in Miami. It will also play a great role in reducing the losses caused by natural calamities such as lightening strikes and wind storms etc as seer in India and Africa [5-6].

The present sources for harnessing power are based on fossil fuels, hydro anc nuclear sources. Power plants using these sources are located at a distance from the loac center, with distance based on the type of fuel used, availability of areas and othe facilities.

The renewable energy sources are based on solar, wind, hydro, geo-thermal anc tidal forms of energy. These sources are generally tapped by PV arrays, wind generatior farms, fuel cells and special machines to harness the power. As the power utilized by the 
consumers is at a fixed A.C. voltage and frequency, most of these systems depend heavily on power electronics to convert the power from $\mathrm{DC}$ or from varying $\mathrm{AC}$ to fixed frequency and voltage AC.

The future trend focuses on integrating these sources along with the conventional sources at load centers. This presents the general concept of a Microgrid, a cluster of internal generation and loads, functioning in synchronism with the main grid. As these sources are located around the load centers, unlike the conventional generation plants, these are comparably smaller in quantity and are distributed among vast geographical areas, hence, are also called distributed resources. However, there lies a subtle difference between the distributed generation and microgrids. Microgrids are combination of resources, which could be non-conventional and distributed, and load centers with certain logic's of operation, whereas distributed generation refers to all kinds of smaller generating systems such as dedicated wind, PV farms, gas turbines, micro generators, fuel cells and combustion generators irrespective of them making and alliance with the load centers [7-8].

\subsection{Grid Integration Challenges with Sustainable Energy Resources}

Benefits of integrating distributed resources with the power system cannot be achieved without solving the technical issues concerning the operation of these resources. The operational challenges which provide roadblocks for integrating the distributed resources with the power system are presented in this section. 
The microgrid and distributed generation present feasible and practical solution to the increasing energy demand. However utilities are cautious in integrating dispersed generating units to their system [9]. Microgrid integration challenges can be broadly classified into two categories:

a.) Technical Challenges: Issues such as safety, security, islanding, restoration from scheduled and unscheduled shut downs, control, stability, protection coordination, capacity and reserve management, reliability and power quality liability, cost development in the needed interconnection technologies are all among the urgent concerns needing to be addressed [10]

b.) Non-Technical Challenges: Issues pertaining to pricing incentives, decision priority, risk responsibility and insurance for new technologies adaptation, interconnection standards and regulatory control and addressing barriers.

The following sections present the technical and non-technical challenges from the perspective of utility company and Independent Power Producer (IPP) [11].

\subsubsection{Issues affecting the Main Grid Performance}

1.) Control Problem: As discussed earlier the normal operation of the power system depends on the satisfaction of the various network constraints. However, the integration of microgrids and distributed generations increases the complexity. In case of a conventional power system the constraints and variables of operation are controlled by the utility operators. In case of a power system with microgrids and distributed generation not just the power system but also the microgrid should satisfy the constraints of the individual network as well the mutual constraints at the point of integration. This 
poses new challenges as the network constraints such as voltage and frequency are to be maintained not just by the power system utility but also by the microgrid.

2.) Scheduling: In centralized power management, scheduling is an important factor for the reliability of the energy supply. Integrated operation of distributed resources puts a concern on this issue with respect to the utility company as most of the non-conventional sources are non-dispatchable sources such as wind and PV array. In case of these systems a special scheduling and dispatching terminology is required.

3.) Protection: Traditionally electricity distribution allows a unidirectional power flow and the feeders are protected with unidirectional protection gear. MG can be integrated with distribution network in two distinctive modes. In the first mode the MG only draws the difference of local generated power and local loads and during any disturbance isolates itself as an Islanded power system. In this mode there is no injection of power into the main grid. Second mode is grid connected power injection and power drawing mode where the MG feeds the excess generated power into the main grid, or draws from the microgrid during deficiency. In this case the power flow is bidirectional, concerning about requirements of a manipulated distribution system to allow $\mathrm{MG}$ operation. Also in case of interconnection protection and communication infrastructure functionality and reliability have to be addressed by both utility and IPP. Relays are normally used to provide zonal protection and are independently protecting each zone without any cross-communication between the zones. After integration a dynamic communication layer is required or intelligent agents are required to assess the situation for decision making. 
4.) Transmission Congestion: In the present system, the economic dispatch also considers the losses in the transmission lines to balance the load and demand, with the integration of MGs this issue has to be addressed. Also the congestion and loading limits of the transmission lines are to be considered.

5.) DG Penetration: The maximum penetration level of the DG which will let the utility compromise on the stability and reliability has been estimated up to $25 \%$ by many researchers.

6.) Cost Sharing: The commercial viability of the microgrid concept should be mutually beneficial for the IPP and also for the utility company. The profit and loss sharing as well as investment costs need to be assessed in a suitable way. Generally some microgrid models deploying wind generators draw reactive power from the main grid even in case of grid synchronized power injection mode. There needs to be a strategy to properly distribute the profits which requires real time online calculation. Another problem faced is the dispatching and scheduling of power from the MGs. As most of the MG sources are non-dispatchable it creates a problem for the utility company for unit commitment and dispatch. There have been some efforts to address the unit commitment problems in microgrids [12] however they are needed to be tested in a real time environment and are present analysis work is rudimentary.

As solutions are being developed for the above mentioned concerns, there needs to be a platform to verify the solutions and assess their reliability before implementing them in the present power system. 


\subsubsection{Issues for Microgrid Energy Sharing and Power Quality}

1.) Voltage and Frequency Management: In case of grid connected operation, the microgrid considers the frequency and voltage of the main grid for reference. The microgrid in this case should continue to support the grid by following the dynamic variations. However, more important concern is during the islanded operation of the Microgrid. One or more primary or intermediate sources within the microgrid should form a grid and establish the voltage and frequency. Non-dispatchable sources provide a concern here as they do not have any inertia and cannot supply to the dynamic variations in the load. This can generally be overcome by using storage and intermediate resources such as flywheel or combustion generators.

2.) Balance between supply and demand: If the microgrid was exporting or importing power to the grid before islanding, then control action should be implemented to balance generation and demand. If the connected load exceeds the available generation, load management should be implemented.

3.) Power Quality: As the microgrid employs power electronic based generators, the power quality is another constraint. The MG should maintain the harmonics within the limits during the grid connected and islanded mode.

4.) Communication within Microgrid Components: A major concern is the communication within the various microgrid components. If the communication layer is unavailable or unreliable then the control and protection logic will cease to exist in a Microgrid. The communication layer should connect the generation sources, loads, control systems and protection system to alleviate the problems during islanded mode of operation. 


\subsection{Statement of the Problem}

The purpose of this thesis is to develop operational strategies for implementing power system test bed with AC generating stations emulating power plants, transmission and distribution systems through transmission line and bus bar models, constant current, constant impedance and constant power loads models emulating load centers, communication and data acquisition layers and control layer interconnected with sustainable energy resource emulators interconnected to form microgrid integrated with the conventional AC system. This platform is hence comprised of AC generating stations, transmission and distribution system using lines and cable emulators, dynamic and controllable loads, energy control and management systems, and data acquisition and communication systems representing the modern day power system. Along with this, the platform also contains microgrids, with internal generation comprising of renewable or non-conventional sources of power such as wind, PV arrays, fuel cells and combustion generators. The purpose behind the integration of microgrids in this energy system test bed is to determine the effects of the integration of renewable energy sources on the existing AC power system. This will help in determining the efficiency of the power electronic converters, the control system, control algorithms, and will act as a framework for testing various control algorithms, communications layers, power electronic converters and source simulations. The power system test bed overall is to represent the state of the modern day power system with the addition of microgrids and distributed generation. The basis of this work is Dr. Mohammed's idea of power system test bed and microgrid platform [13]. 


\subsection{Contribution of Thesis}

The power system and microgrid platform developed in this thesis will enable the researchers to study the dynamics of the present power system with the integration of microgrids and hence improve the overall system. To study power system operational issues in the presence of distributed renewable energy sources.

There are four main areas of application of this research. The first one helps in determining the security and the stability of the present power system to handle the microgrids, smart grids and distributed generation. Different sources or simulations of sources can be implemented to test the response of the system. This helps the national power grid to evolve from a centralized generation and control architecture towards a distributed and decentralized architecture. The second area of concern is the implementation of control architecture, the work in this thesis acts as a platform to conduct research on different control methodologies and to verify the logic on actual power system. The third area of concern is the optimization of power, the present plat form provides a unique opportunity to develop and implement logic to optimize the power in the system with the integration of non-conventional sources. The present system of economic dispatch and unit commitment is based on a centralized approach and load prediction is determined based on a large scale area. This system will help in implementing prediction and optimization techniques on a hierarchical level. The last area is to implement new techniques for power system protection. Present power system architecture is based on the direction of power flow, voltages and frequency, and is overall one dimensional with consideration on voltage, frequency and power from the 
main grid. With the advent of microgrids, the direction of the power, voltage and frequency during islanded and synchronized operation varies, and hence the protection architecture could be modified to adapt to these constraints. The platform developed in this thesis will help in implementing and studying all the above characteristics and in the development of better techniques to solve the issues related to integration of microgrids.

\subsection{Organization of the Thesis}

The thesis is organized into 11 chapters. Chapter 2 presents the sustainable energy resource emulators developed for integration with the AC power system on the test bed. Chapter 3 presents the development of components for the AC test bed. This includes the generating stations emulated by induction motor driven alternator, transmission line and bus bar modules, synchronization module and block diagram for the desired test bed. Chapter 4 presents the implementation details of the components of the test bed. The various architectures in which the test bed can be configured are also presented in this chapter. Chapter 5 presents the integration of alternate energy resources to emulate a microgrid and their interconnection with the AC power system on the test bed. Chapter 6 describes the communication layer developed for inter component data transfer and monitoring on the test bed for control and data logging. Chapter 7 presents the experiments performed on the test bed with experimental setup and results. A section is also included to analyze the results obtained for the experiments. Chapter 8 presents the practical implementation and educational impact details of the test bed. Chapter 9 presents the discussion of overall research conducted as a part of this work followed by the conclusion in Chapter 10 and future work in chapter 11. 


\section{SUSTAINABLE ENERGY SOURCES}

\subsection{PV Array Emulator}

Photovoltaic arrays are a composition of photovoltaic (PV) cells in a series parallel arrangement. The number of cells and their arrangement determine the current or the voltage that is available. Generally for simulations, the PV arrays are modeled using an equivalent one diode model to represent a solar cell, assuming the energy input source as a voltage controlled current source.

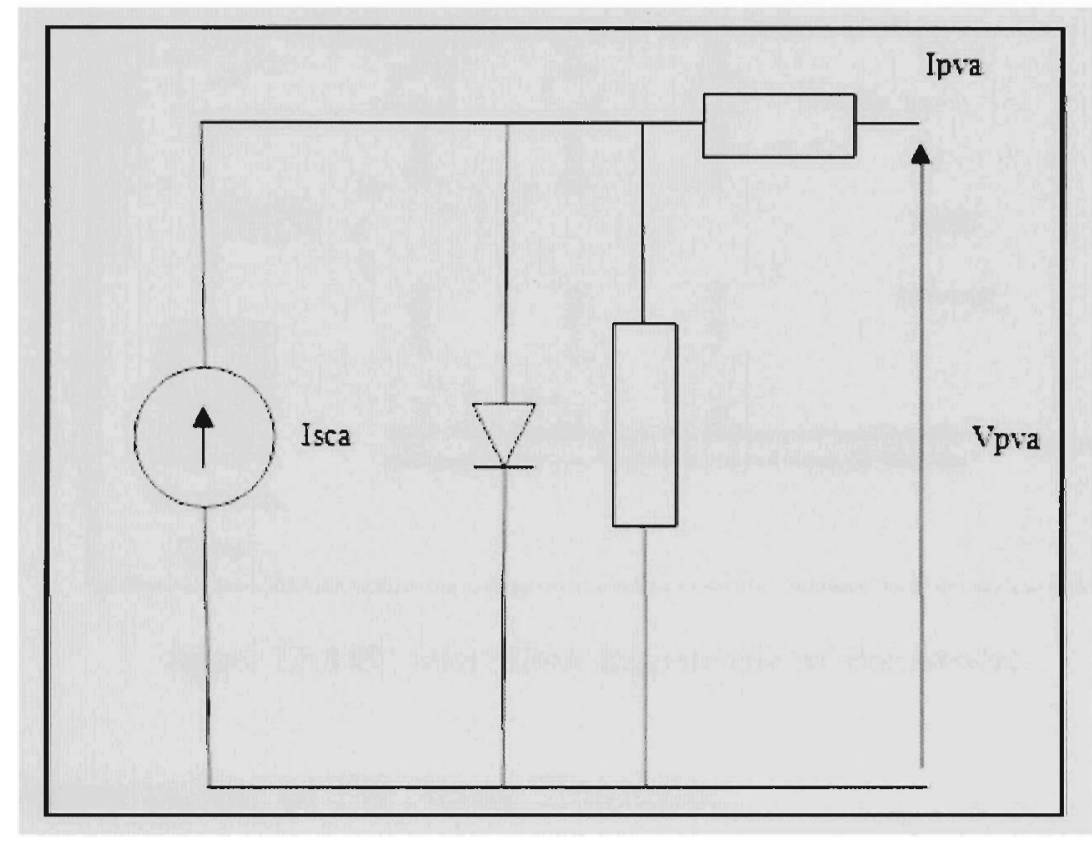

Figure 2.1-1 Circuit Diagram representing a PV Array equivalent circuit

Where, Ipva is DC current output from the array, Isca is the short circuit current at room temperature and Vpva is the DC output voltage of the array.

As the power from the PV array fluctuates with the change in intensity of light, these arrays are connected to power electronic interface devices. These devices implement the Maximum Power Point Tracking (MPPT) algorithm to extract maximum power from the array at the operating voltage and current. These arrays are generally not 
directly connected to the loads due to the unreliability of the power source, hence they are connected to storage systems and loads in parallel. Dump loads, which are basically heating loads are also connected in some case and are applied in case of saturation of storage system and unavailability of load. The figure below represents a PV array based system.

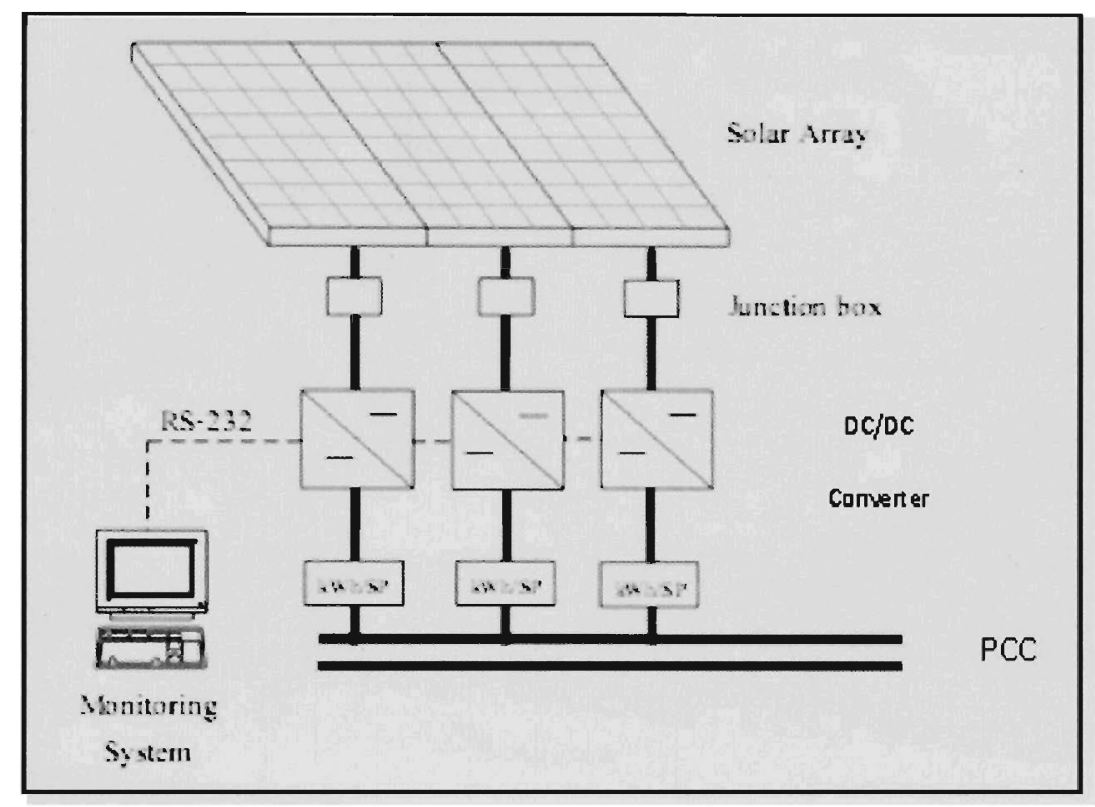

Figure 2.1-2 PV Array block diagram and interface model.

\subsubsection{Implementation of PV Array Emulator}

The basic operation characteristics of a PV array have been presented in the preceding section. As the response of the PV array depends on the solar energy, it was considered a better option to simulate a PV array system than designing an actual PV array. There are basically two ways in which the PV array can be implemented.

The PV array model can be implemented by utilizing a 42V DC permanent magnet motor Solver motor generator set of 250 Watts capacity. An experiment was conducted to verify the viability of this design. The DC motor generator set was connected to a Grid Tie 
Inverter and loaded. The results and the experimental setup are displayed in the figure's

\section{1 .3 and 2.1 .4 respectively.}

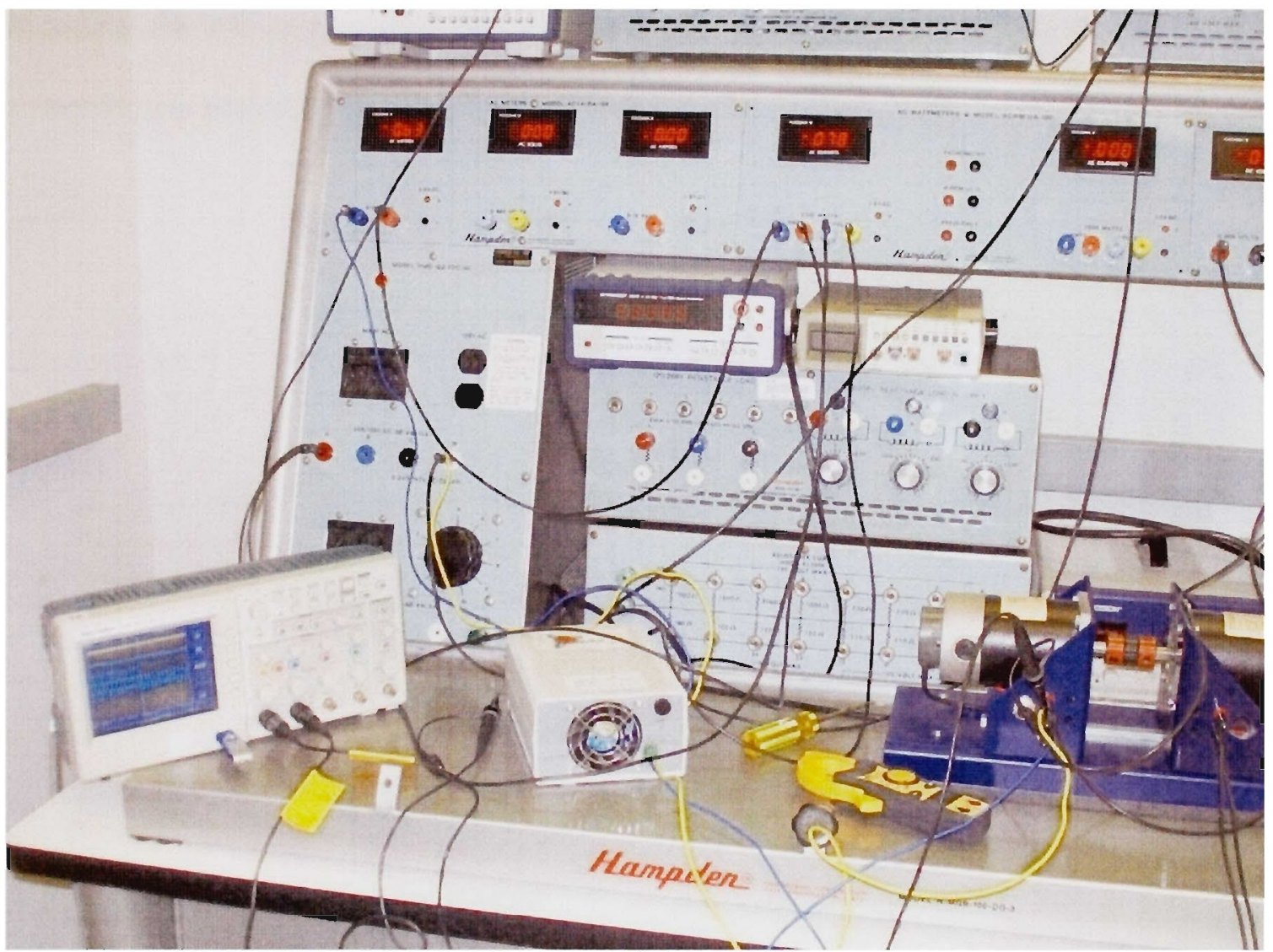

Figure 2.1-3 Experimental set up for PV array emulator using Motor Generator set

It has been observed that voltage and power output of the set can be adjusted by adjusting the DC motor speed, this variation causes a change in the DC generator output. The power electronic converter utilizes a MPPT algorithm to set the operating point based on the voltage. This system can also be extrapolated by using a Hampden motor generator set. The benefit in this case is that operation voltage is higher and access to field of both the machines is available which provides a separate control for voltage output and power output. A road block was encountered however as a grid tie inverter 
(GTI) was unavailable for $120 \mathrm{~V}$ DC input. This can be implemented in the future with a Semikron power electronic module based grid tie inverter.

Secondly the PV array model can also be implemented by using a programmable DC power supply providing a cost effective solution.

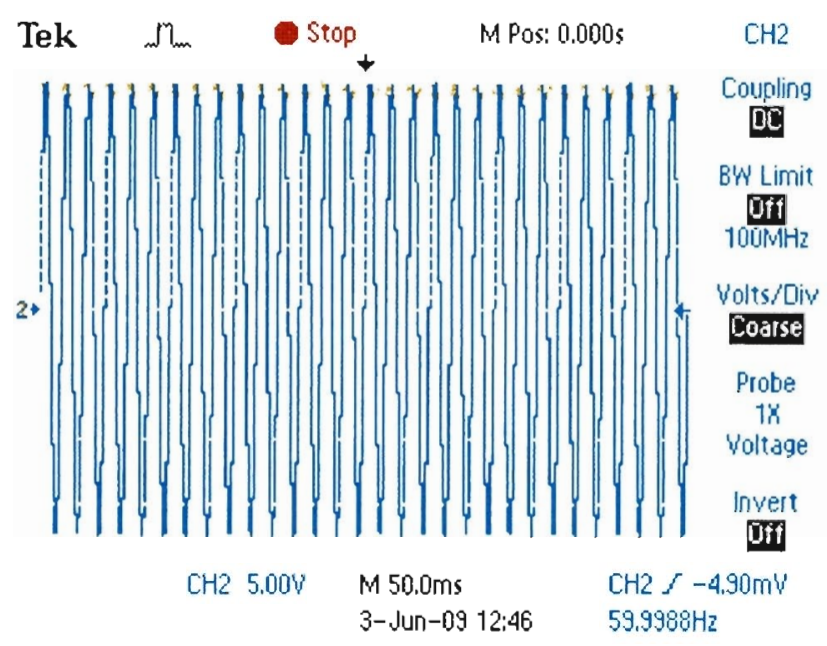

Figure 2.1-4 DC Voltage output of the DC generator and AC output of the Grid Tie Inverter

\subsection{Wind Energy Emulator}

\subsubsection{Model for Wind Energy Emulator}

Wind has been used as a source of energy for a long time now but it has been only in the last century that it has been used to generate electricity and only recently with the development in power electronics, mechanical and electric machines technologies, it has become economically feasible to use wind for electricity generation. Microgrid and Distributed generation concepts have led to a renewed interest in Wind energy from the utility companies. Although wind currently accounts for less than two percent of global electricity production, the installed wind capacity is growing rapidly. There are steady gains in Europe on deployment of wind energy based generators. Recently, a large 
market for wind energy has developed in Asia, particularly in India and China. This makes wind a suitable model for non-conventional electrical energy resource and makes it an important component to include as a part of the Power System Test Platform. Power in the wind is extracted by wind turbines, which generally vary between large turbines generating more than $1000 \mathrm{~kW}$ of power to microturbines generating up to $1 \mathrm{~kW}$ of power.

The Wind emulators are modeled by using a DC Motor to emulate the wind by variation in speed and torque, this motor is coupled to a generator, as a synchronous machine functions at constant frequency Induction machines are used as generators. The reactive power to the induction generator is provided by the capacitor bank or main grid. The block diagram in figure 2.2-1 represents a typical wind emulator system.

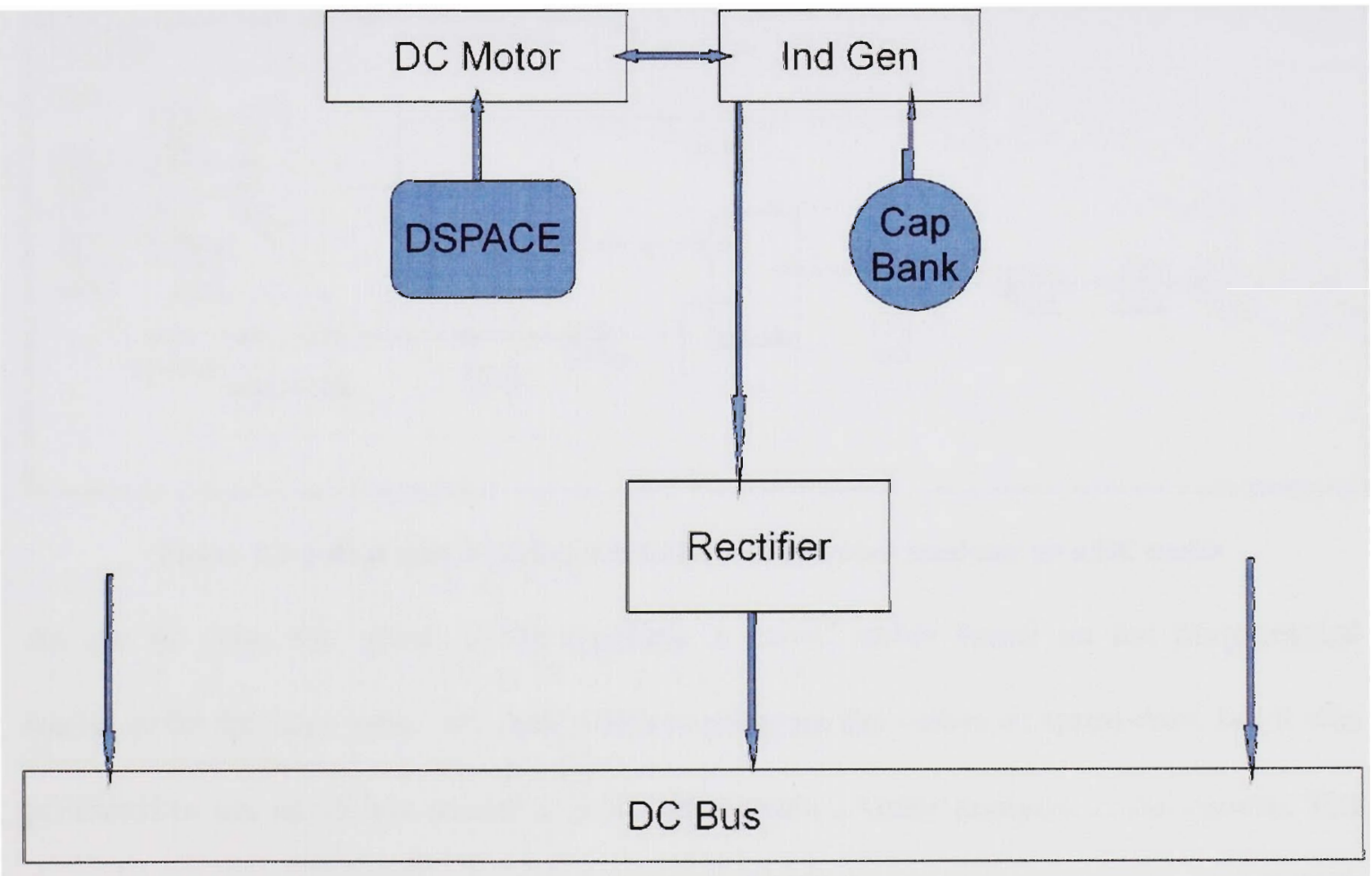

Figure 2.2-1 Wind Energy Emulator Block Diagram 


\subsubsection{Implementation of Wind Emulator on 120 Volts and 42 Volts DC Machine and Induction Generator set}

The Wind emulator is an important part of the non-conventional energy source utilized in the microgrid. There are three major parts of this emulator, namely, Wind Speed emulator, Wind generator and power electronic interface system. The modeling of the wind turbine system as a part of this thesis is based on [14-15] DC Motor and Induction Generator model, emulating wind variations and generator response.

The Wind Speed emulator has been implemented on a DC motor. A real time Simulink model has been designed in Matlab to implement the speed variation. The model is displayed in the figure $2.2-2$.

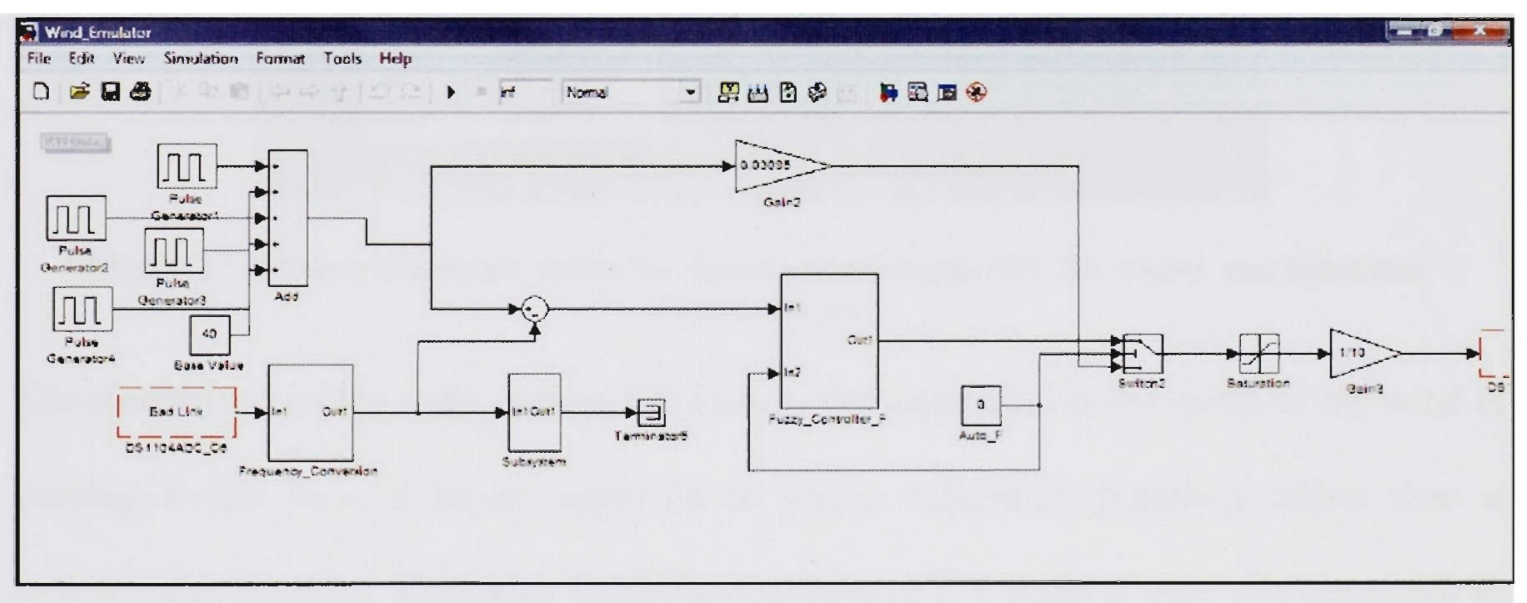

Figure 2.2-2 Real time Simulink model for wind turbine emulator on a DC motor

As can be seen, the speed of the machine is varied either based on the programmed model or by the user input. An option was to program the values of speed data, but it was preferred to use an ad hoc model as it would provide a better analysis of the system. The Simulink model logic is implemented by the dSPACE 1104 control board. A KBPWS drive was used for controlling the speed of the DC motor. This is a power electronic rectifier, which works on PWM (pulse width modulation) through a control voltage of 0- 
$5 \mathrm{~V}$ DC. This control voltage is responsible for DC output of the rectifier varying from 0$120 \mathrm{~V}$ DC. Figure 2.2-3 presents a picture of the speed control system of the DC motor.

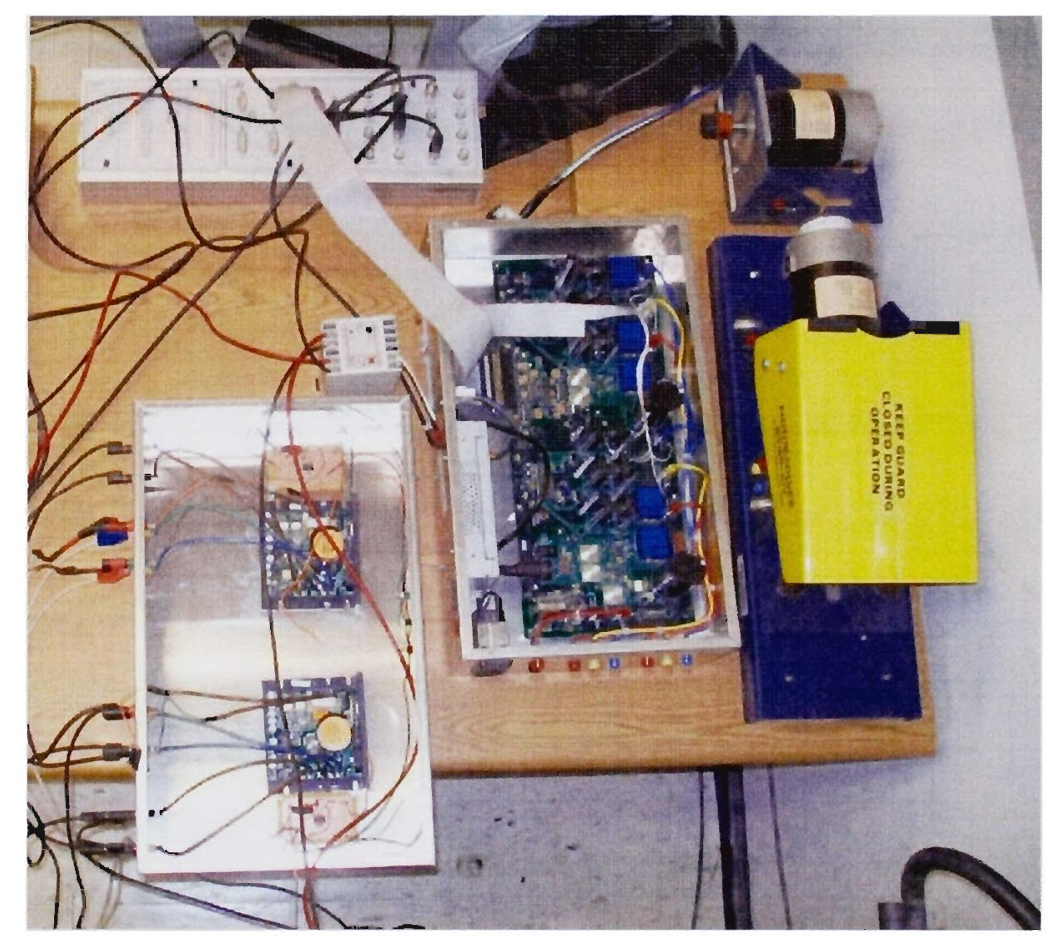

Figure 2.2-3 Power electronic drives for Speed variation and 42V DC Motor and Induction Generator set

The second part of the wind turbine emulator is the generator, as the speed of the wind is varying it has been a better approach to utilize induction generator rather than a synchronous machine. Hampden induction machine of 250 watts is used as a generator in this model. The induction machine does not have any explicit field. In its operation as a motor the induced eddy currents in the rotor generate a field for the induction machine. However for its operation as a generator it requires an additional field for voltage build up. There are two ways in which this can be provided. The machine can be started as a motor and then a prime mover can drive the induction machine to make it operate as a generator in this case the induction generator draws the reactive power from the main 
grid. The problem in this case is that machine will always rotate at a constant frequency and fall into the motoring mode once the speed of the prime mover falls below the speed pertaining to the operating frequency of the grid. The other option is to use capacitors to provide the reactive power to the field, as the capacitors store voltage they provide for eddy current generation in the rotor. It is a better way of providing reactive power as the machine can operate at any frequency without any trouble of entering the motoring mode. A capacitor based charging module was developed to provide the reactive power to the induction generator used for this experiment. The capacitor charging module and the picture of the generator is presented in the figure 2.2-4.

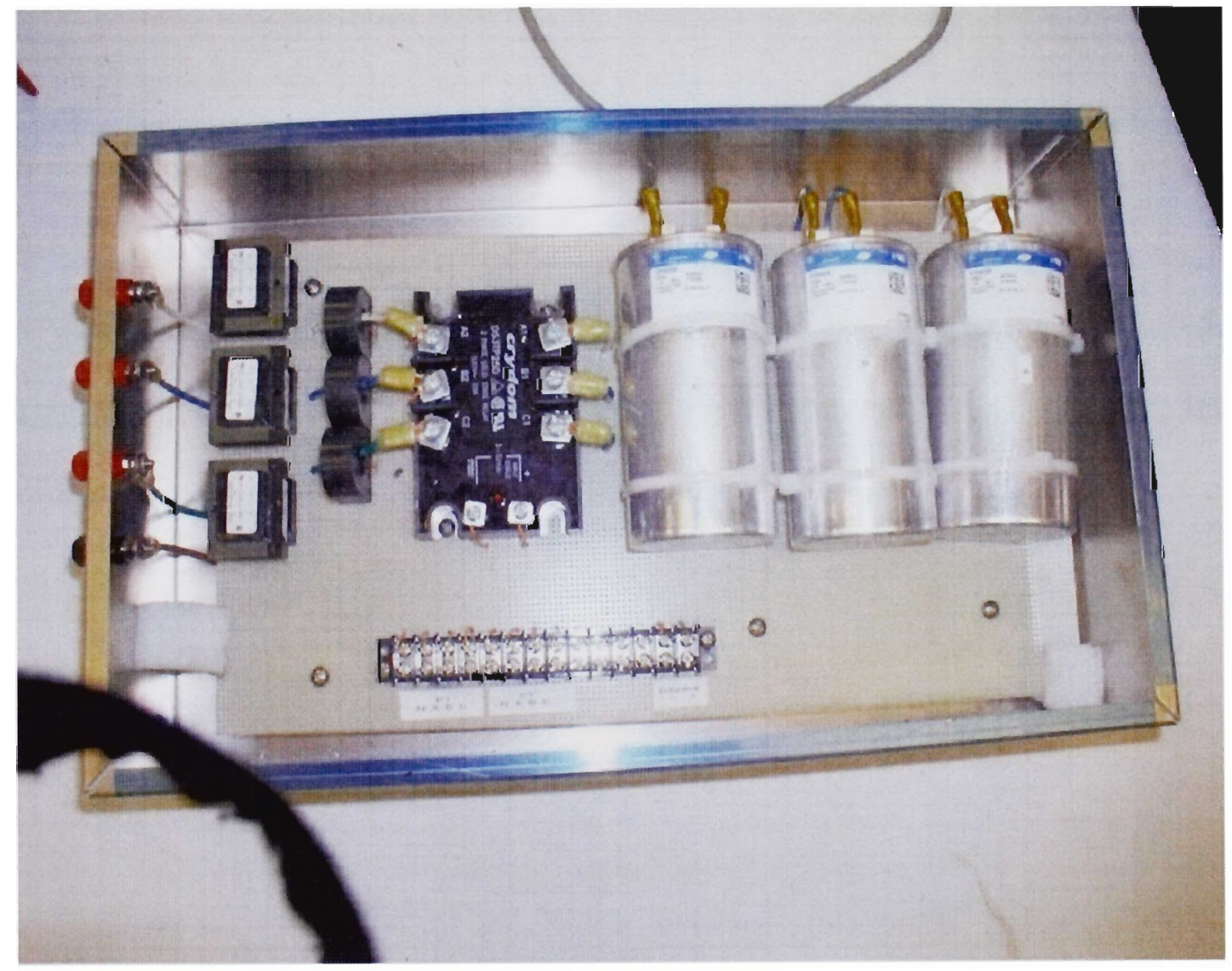

Figure 2.2-4 Capacitor based field excitation module for Induction generator 


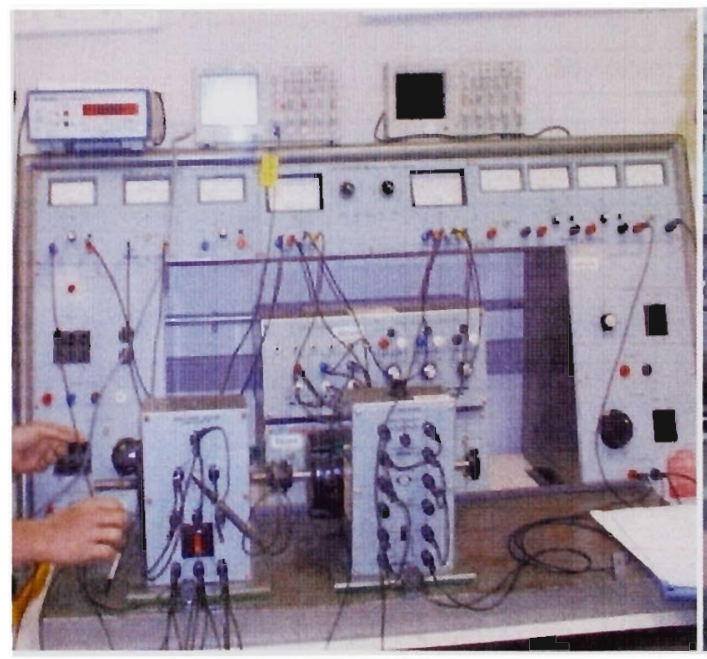

Figure 2.2-5 Hampden Induction Generator

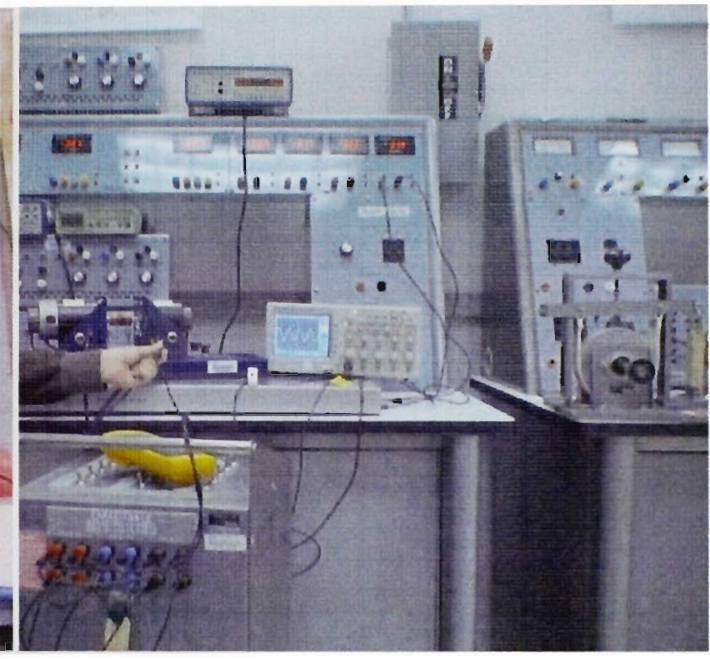

Figure 2.2-6 Motor solver Induction Generator

An experiment was conducted to verify the response of the induction generator and DC motor set to verify the output and characteristics. It was performed on $208 \mathrm{~V}$ and $42 \mathrm{~V}$ machines. The figures 2.2-5 and 2.2-6 display the experimental setup for both of these cases.

The results of the $42 \mathrm{~V}$ induction motor generator set are presented in the figures $2.2-7$, 2.2-8 and 2.2-9. The $208 \mathrm{~V}$ machine also provided similar results and they have been recorded on multi meters.

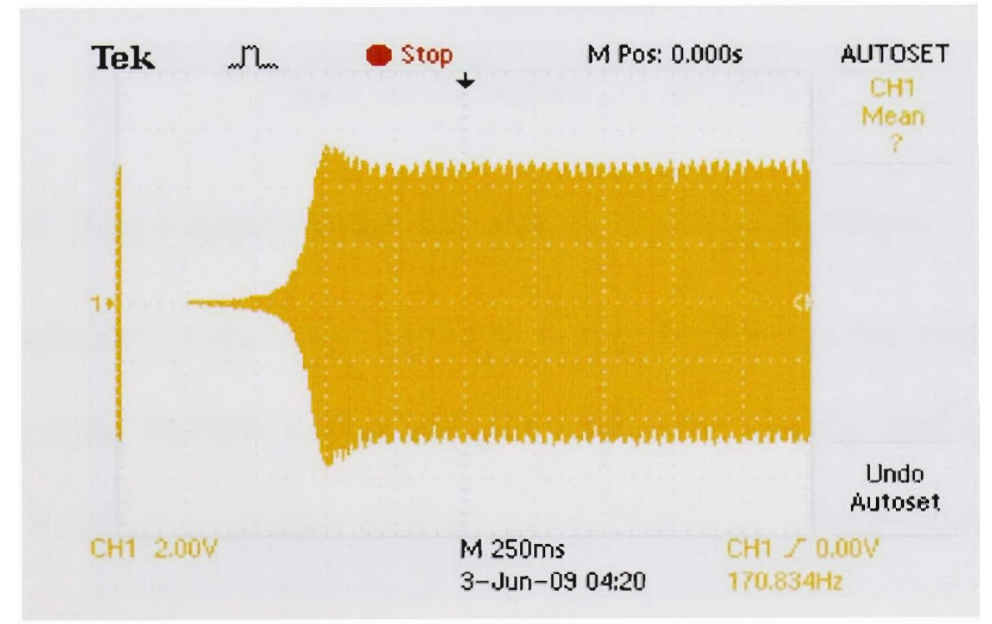

Figure 2.2-7 Start up characteristics of Induction Generator 


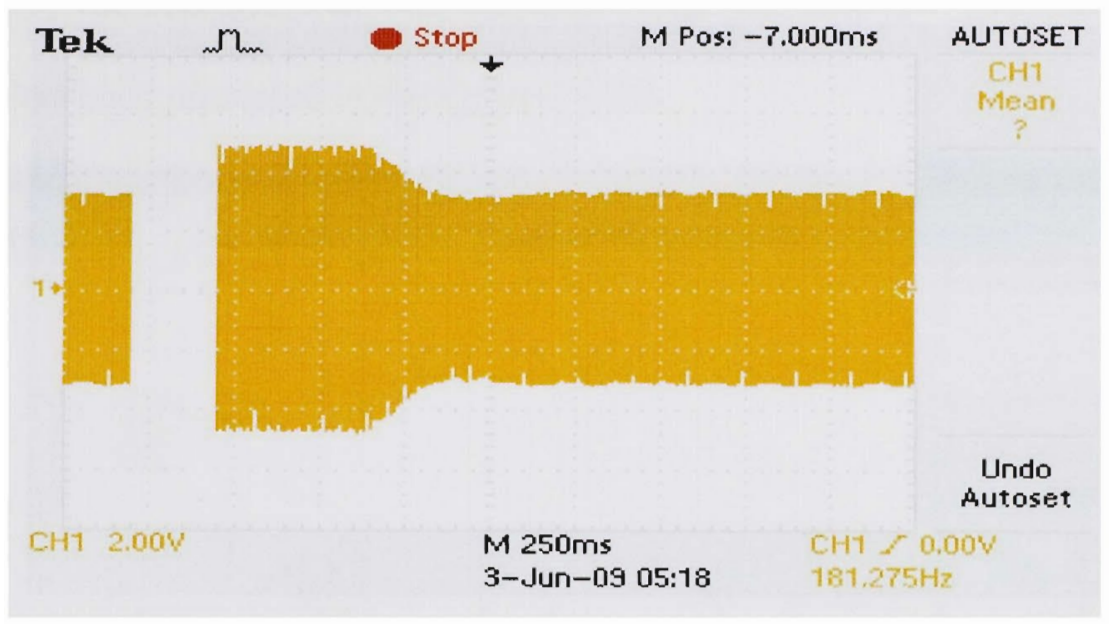

Figure 2.2-8 Loading event

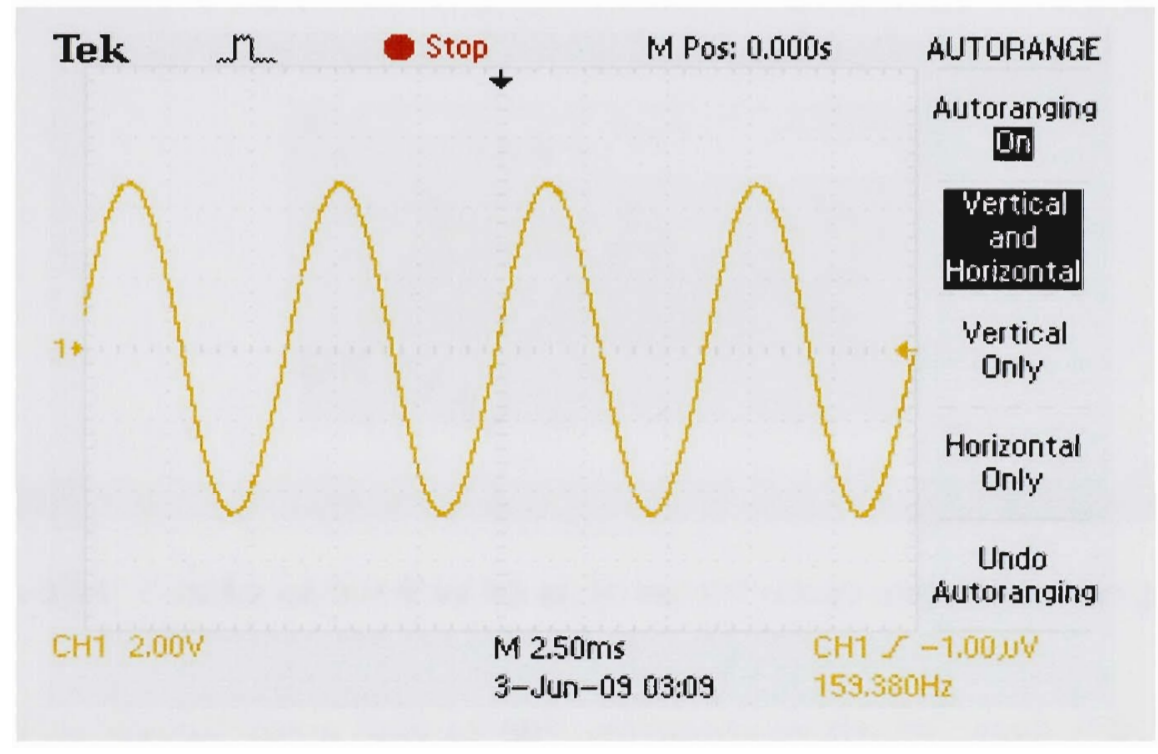

Figure 2.2-9 Frequency of operation

\subsubsection{AC-DC Buck Converter for Wind Energy System}

The final part in the wind emulator is the rectifier, as the output frequency and voltage of the wind turbine is fluctuating it cannot be directly tied to the grid. It was considered to develop a rectifier module using Semikron power diode based bridge. A 
simulation of the rectifier was first done to verify the concept. The Simulink model developed has been presented in the figure 2.2-10.

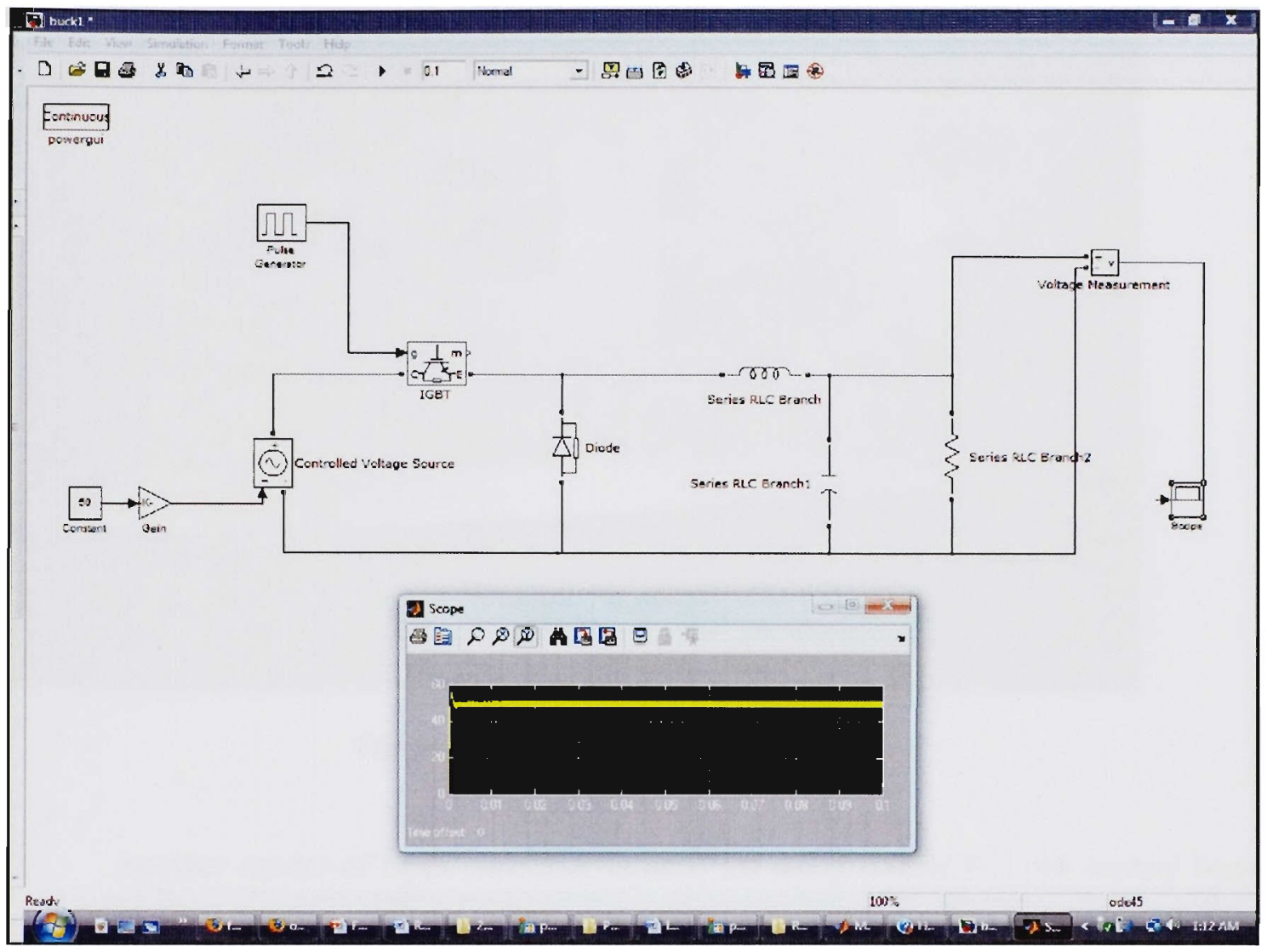

Figure 2.2-10 Rectifier module Simulink model and DC voltage output waveform graph

As the grid tie inverter has a limit of $60 \mathrm{~V}$ maximum on the DC input side, the firing angle for the buck converter was adjusted to provide a 50V DC output from the rectifier module. It can be observed from the graph in figure above that the output remains within the prescribed range.

Hardware module was later developed and an L-C filter was also used to reduce the ripple content in the DC output. The figure 2.2-11 presents the hardware rectifier module. 


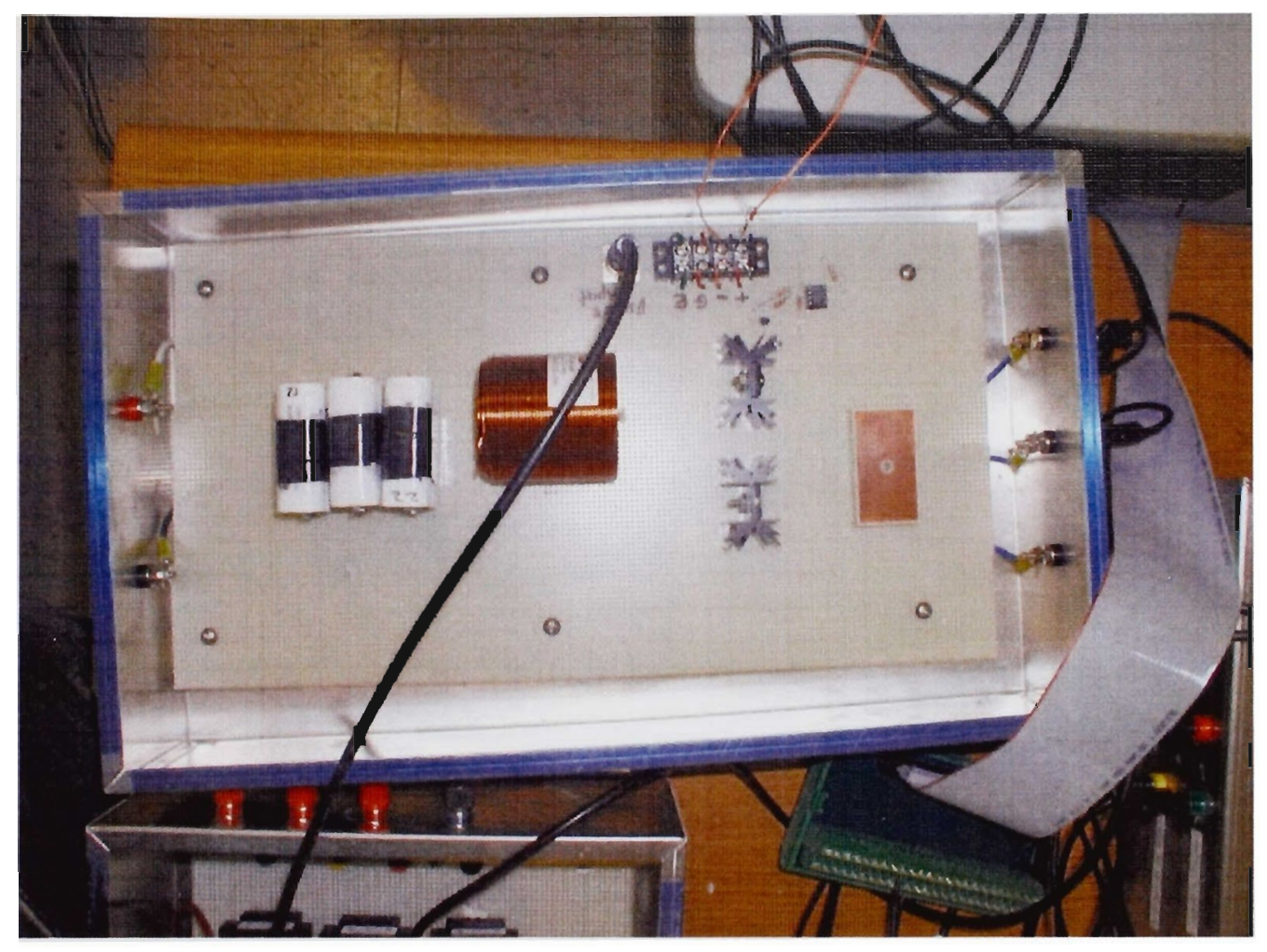

Figure 2.2-11 Rectifier module with L-C filter

Another model of buck converter is used for using dSPACE 1104 control board presented in figure 2.2-13. It provides faster control and reduces the spikes on the output DC Voltage. Semikron diode rectifier module is used with an IGBT and LC filter for controlling the DC Voltage. The Simulink block used for control is presented along with the hardware module in the figures 2.2-12 and 2.2-13. 


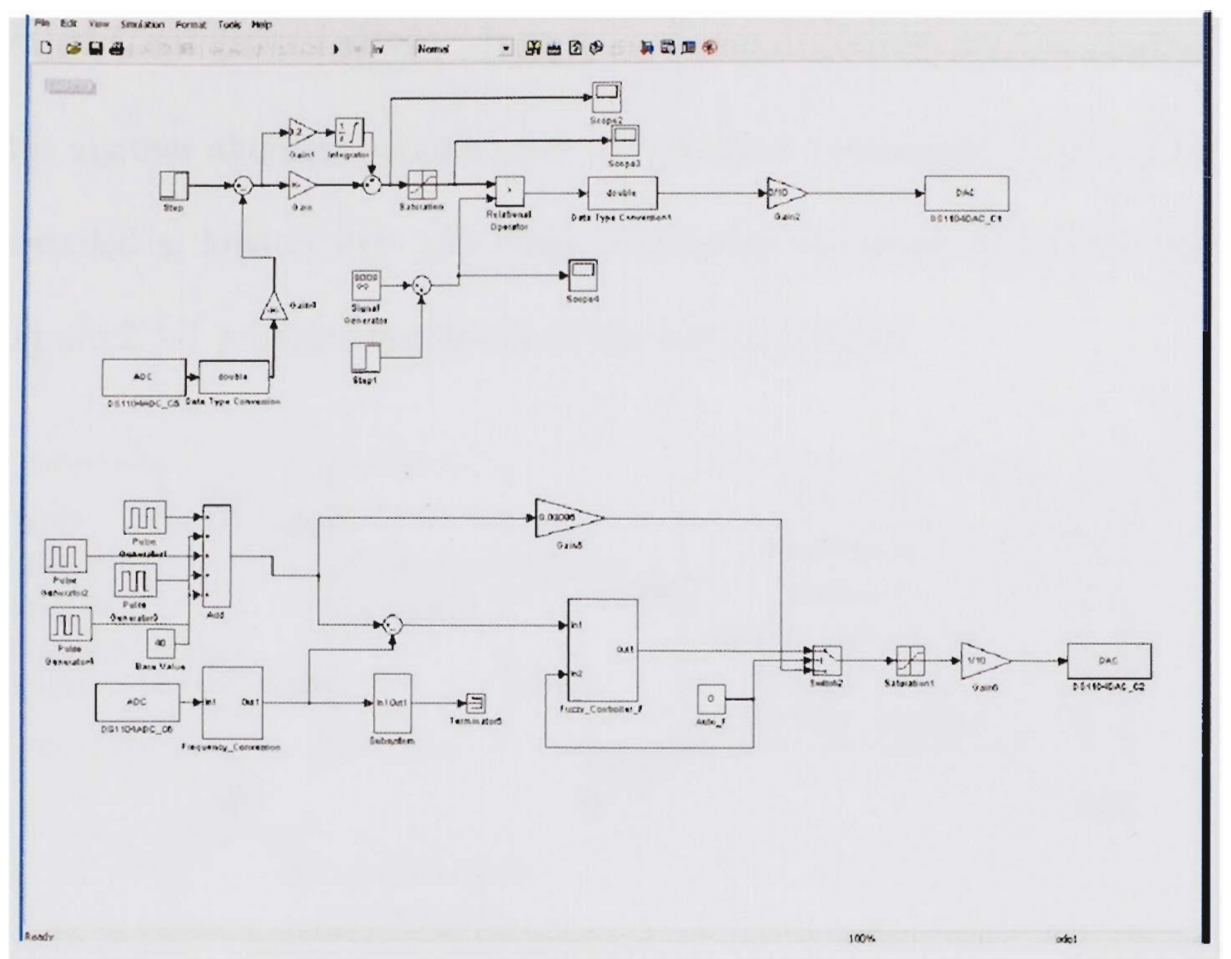

Figure 2.2-12 dSPACE/Simulink Buck Converter module for Wind Energy Generator

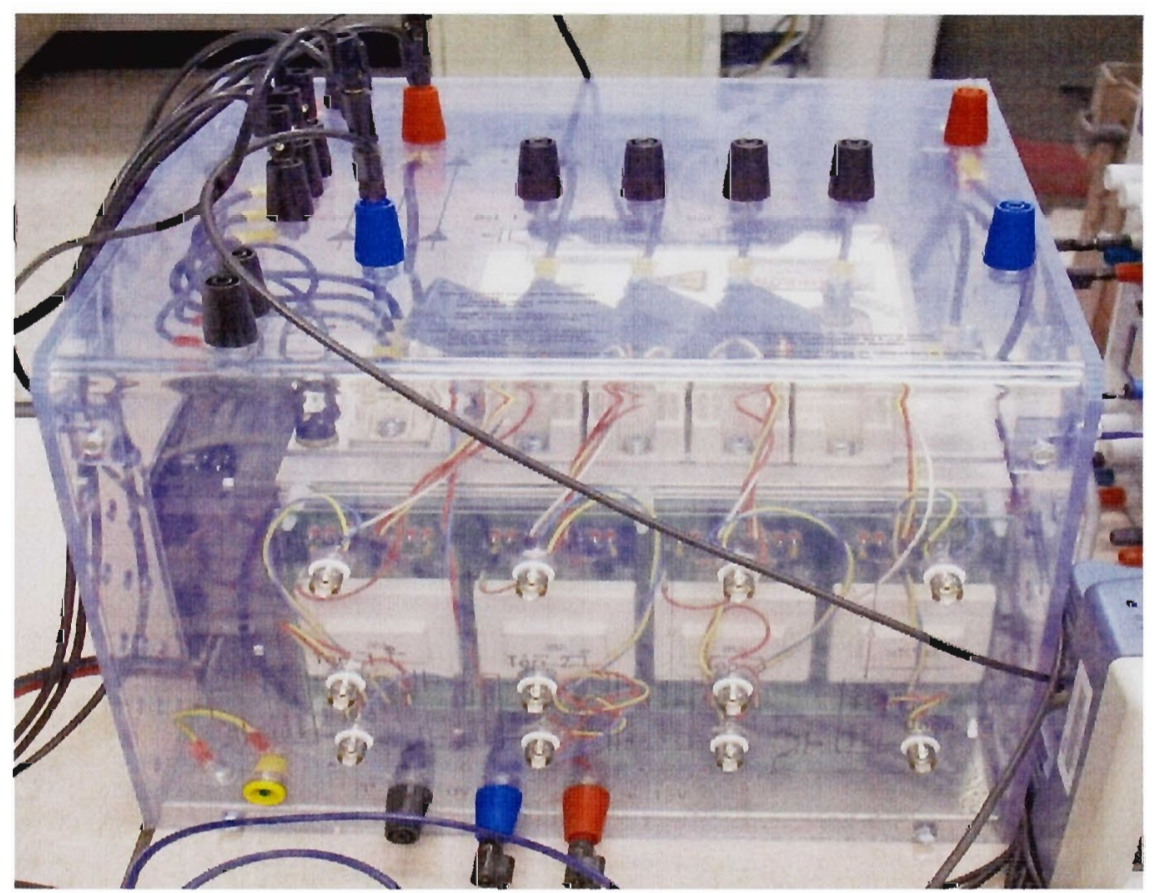

Figure 2.2-13 Semikron AC/DC Buck Converter for Wind Generator 


\subsection{Fuel Cell Emulator}

Fuel cell is another alternate resource and it's a critical component of the microgrid as it can be installed at load centers and hence increasing the reliability of the system. The block diagram 2.3-1 provides the details of the fuel cell model

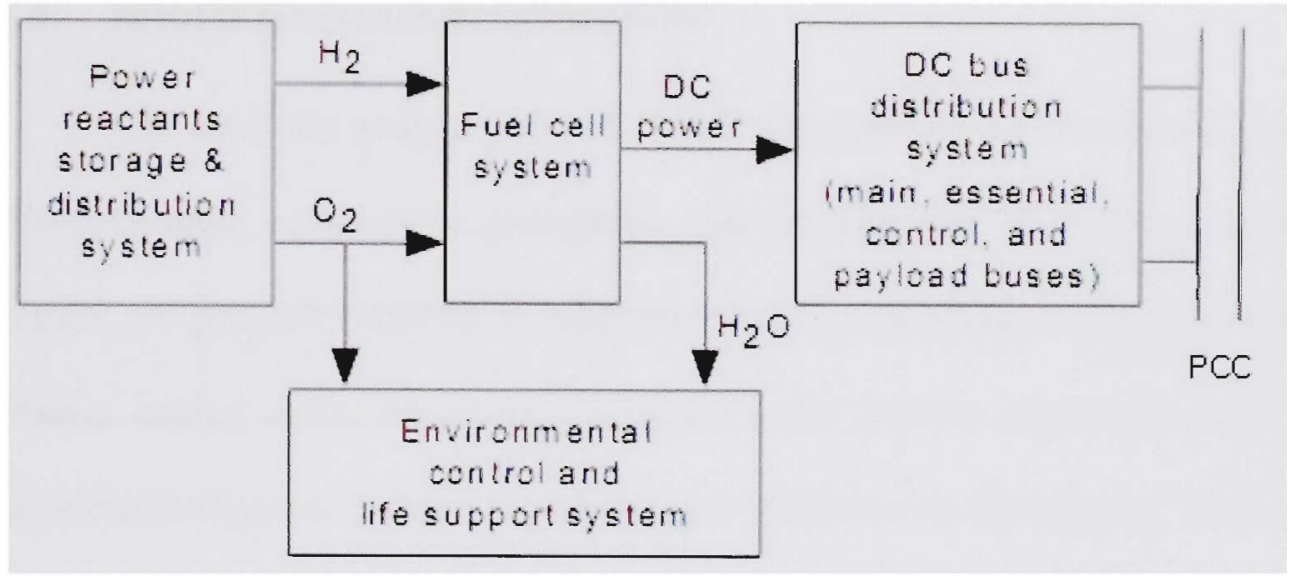

Figure 2.3-1 Fuel Cell Block Diagram

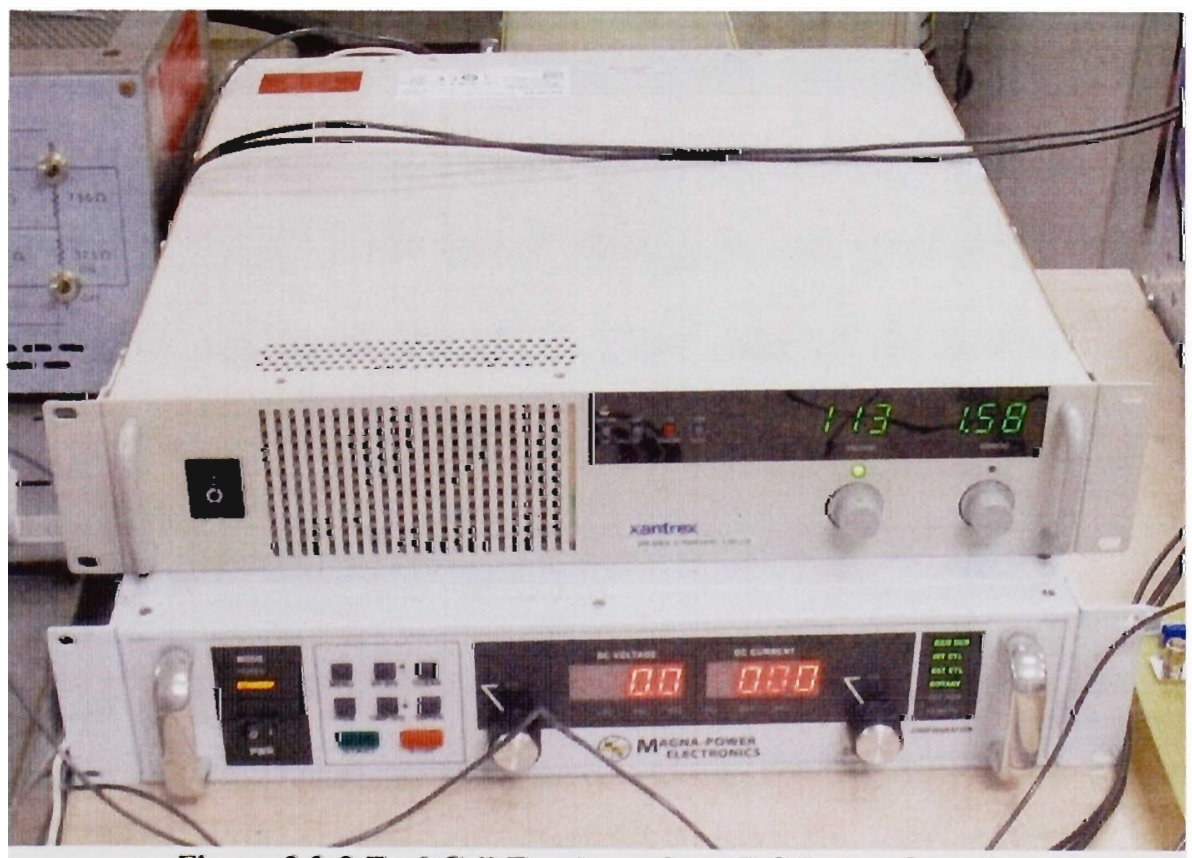

Figure 2.3-2 Fuel Cell Emulator from DC Power S upply 
Fuel cell emulator is implemented through a programmable DC power supply on the test bed. The voltage and current can be programmed in the power supply with pre determined variations recorded from an actual fuel cell. The figure 2.3-2 the DC power supply used for the fuel cell variation.

\subsection{Micro Generator Emulator}

The auxiliary sources utilized in the microgrid or for distributed generation are Micro turbines, combustion generators, gas turbines and oil based generators. These sources are generally applied to meet the dynamic variations in the load as they have a rotating energy unlike the power electronic based devices. Moreover these sources are dispatchable sources and hence also provide reliability to the system. These sources are also used for reference in case of an islanded operation. Generally either the largest generator is used for reference or a combination of large generators is used based on application criterion. Although these are technically conventional generators, the gas turbines and combustion generators can be run by bio gas and others such fuels instead of the fossil fuel. The benefit of utilizing oil based generators is for reliability concerns and quick response in case of an outage, as black start of the system relies on these generators.

Synchronous machine is coupled with a DC motor to emulate a Microgenerator set. The speed of the DC Machine acting as a prime mover and field of the synchronous machine are controlled using power electronic AC-DC drives at $20 \mathrm{kHz}$ switching frequency. Simulink model is developed to control the speed of the DC machine and field of the $\mathrm{AC}$ machine in turn providing frequency control and field voltage control on the 
AC side. A fuzzy logic module is used in the Simulink module to regulate the control voltage of the drives by verifying the feedback voltage with the reference value set by the user [16]. The reference value can be set in terms of operating voltage and frequency on the control desk layout. The Simulink model and dSPACE user interface are presented in figures 2.4-1 and 2.4-2 with the drive boards in figure 2.4-3.

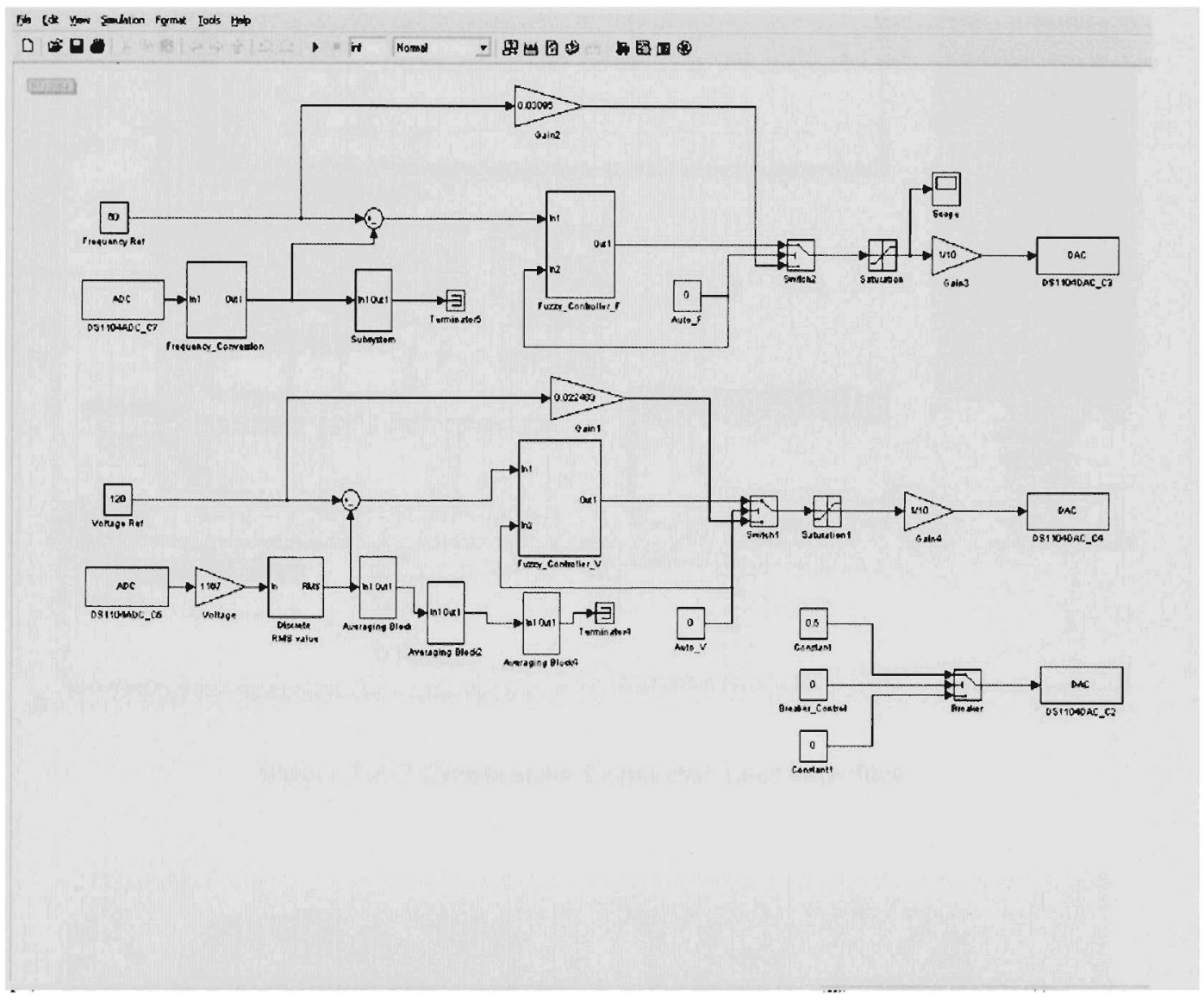

Figure 2.4-1 Simulink Model for Combustion Generator Emulator Control

A control desk layout is developed in dSPACE for monitoring the generator set and providing the reference values for voltage and frequency. The interface can log the voltage and frequency values and helps in catering to the dynamic load variations during islanded operation of the microgrid. 


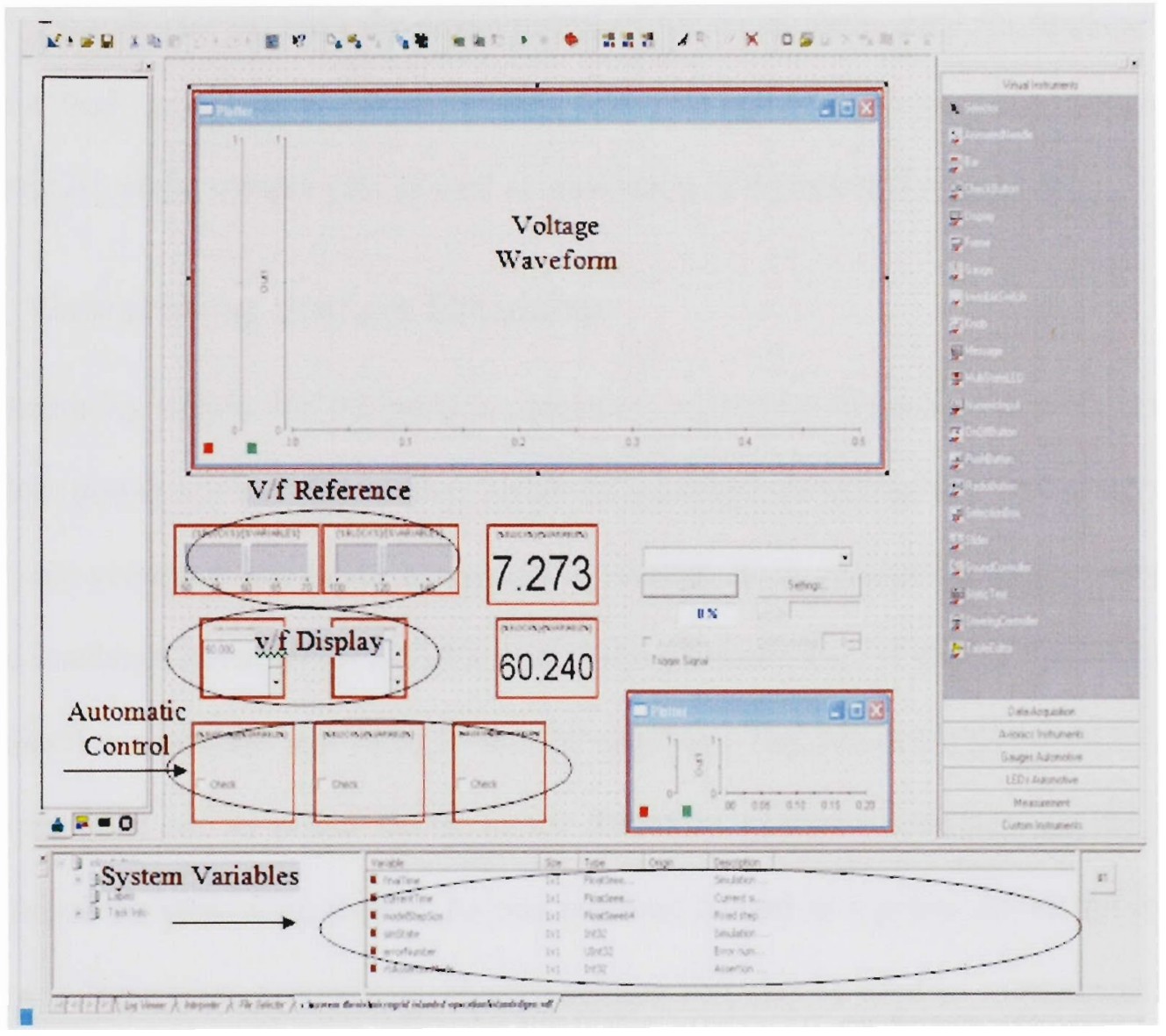

Figure 2.4-2 Combustion Generator User Interface

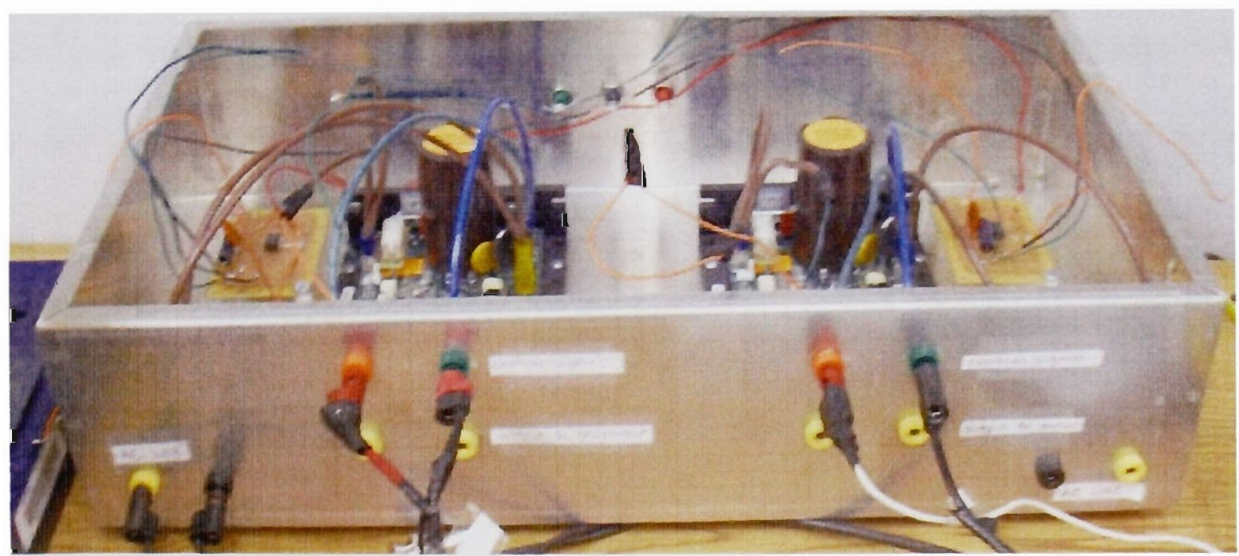

Figure 2.4-3 Power Electronic Drive Boards for DC Motor and Alternator field control 


\section{TEST BED DEVELOPMENT}

This chapter presents the development of the components used for implementing the test bed acting as a power system and microgrid simulator. The design and construction of the components as well as simulation of the test bed is provided.

\subsection{Generating Station Emulator}

The generating station was designed to emulate a real time conventional power plant in a real time power system. The power plants of a modern power system are designed as motor and generator sets called as generating station. Each generating station comprises of five machines mounted on top of a metal base platform. Out of these 5 machines four are Induction machines and there is one DC machine. The primary application of these machines is to act as prime mover to the Stamford generator mounted on the lower plat form of the generating station. As one machine is used as a prime mover during one instance of operation, secondarily, these machines can also be used as mechanical loads emulating pulsed or base loads on the power system. In total there are 5 generating stations, the maximum power output of the generator is determined based on the power rating of the prime mover under utilization. Three of the generating stations have been modeled to provide a maximum power of $5 \mathrm{~kW}$ or $7.5 \mathrm{HP}$ and the rest of the two providing a maximum output of $3 \mathrm{~kW}$. The minimum power operating range of these machines is $1 \mathrm{HP}$. The DC machine provides a maximum power of $2 \mathrm{HP}$ in all the generating stations. The figures 3.1-1 and 3.1-2 present the picture of the generating station. 


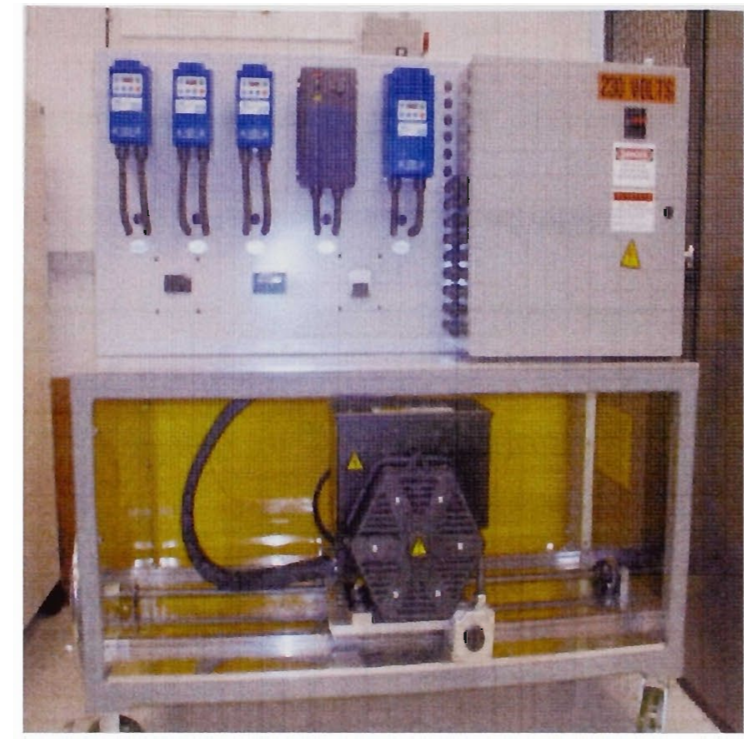

Figure 3.1-1 Generating station front panel

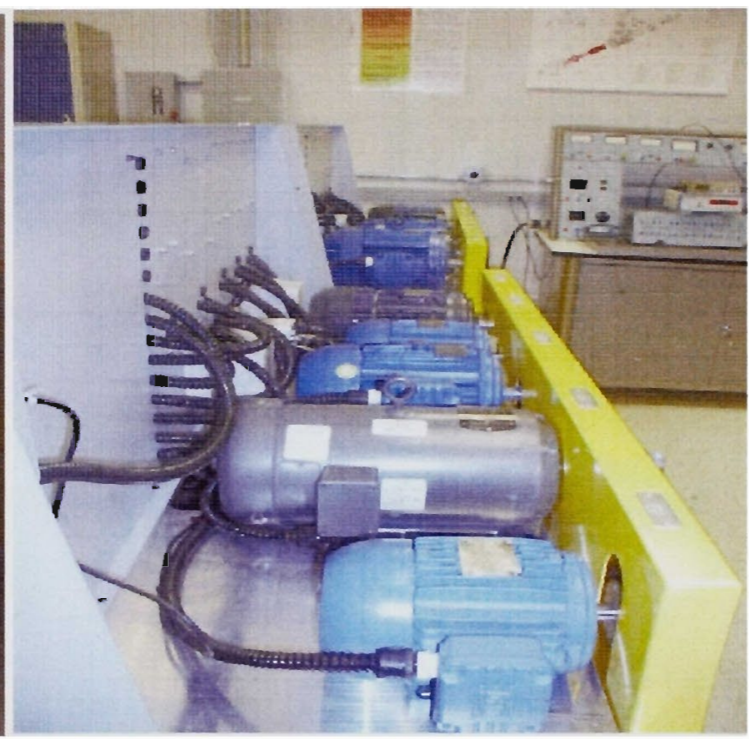

Figure 3.1-2 Generating station side view

An additional auxiliary generating station, Hampden machine desk is also present on the plat form. This station can either be utilized as a load or as a generator with a maximum power interaction capability of $3 \mathrm{~kW}$.

The machine used as a prime mover for the Alternator in the generating station is powered through the FIU power grid. This emulates the conventional energy input in a modern power plant being water, oil or coal. Machines on the upper platform of the generating stations not being utilized as prime mover can be powered from the Energy Systems Lab power grid, which is the power system test bed developed as a part of this thesis, utilizing the power of the generating station, making these machines function as loads.

The Alternator installed in the generating station is manufactured by Stamford and the capacity of the alternator is $10 \mathrm{~kW}$ and hence is upgradeable by increasing the prime mover capacity. However, it has different settings to be operated for different power 
ratings. As a part of this thesis the armature windings of the Alternator are connected as a wye for operation of $5 \mathrm{~kW}$ mode. It can be adjusted to operate in $10 \mathrm{~kW}$ mode by connecting the armature as a double wye. A self excited DC machine installed on the shaft of the Alternator provides the excitation to the generator. Hence the control of the excitation can be done by manipulating the operating characteristics of the DC machine. This control can be obtained through the AVR used on the generator and is discussed in the next chapter.

\subsection{Transmission and Distribution System}

The transmission and distribution system comprises of transmission line and bus bar models developed to emulate scaled down models. The system also employs synchronization module, transformer based lines and data acquisition system embedded into the modules for communication.

\subsubsection{Transmission Line Modules}

The transmission line model is a three phase transmission line simulator operating at 208 Volts. This line model has been built and based on length it simulates three kinds of lines short, medium and long. As the test bed is intended to emulate terrestrial and mobile power system as in ship board and airplanes, the lines have 5 connectors and option to connect neutral to solid ground or leave it floating.

The transmission line model consists of various modules that can be interconnected in different configurations depending on the length being modeled. The transmission line model is constructed using lumped parameters for resistance, inductance and capacitance $[13]$. 
The ABCD parameters for the transmission line model are represented below as a matrix.

$$
\begin{array}{cc}
V S \\
I S
\end{array}=\begin{array}{ccc}
(1+Y Z 1) & (Z 1+Z 2+Y Z 1 Z 2) \\
Y & (1+Y Z 2) & V r \\
\text { Ir }
\end{array}
$$

Where the parameters are as follows,

$$
\begin{gathered}
A=D=(1+Y Z 1) \text { in per unit } \\
B=(Z 1+Z 2+Y Z 1 Z 2) \text { in ohms } \\
C=Y
\end{gathered}
$$

Where $\mathrm{A}, \mathrm{B}, \mathrm{C}$ and $\mathrm{D}$ are the parameters that depend on the transmission-line constants $\mathrm{R}, \mathrm{L}, \mathrm{C}$ and $\mathrm{G} . \mathrm{ABCD}$ parameters can be used to describe the variation of the line voltage with the line loading. The basic line module uses $2.5 \mathrm{ohm}$ at $60 \mathrm{~Hz}$ air core inductors with shunt capacitors to emulate a transmission line. The inductor coils are connected in parallel in a short transmission line emulating 70 miles to reduce the resistance and line to ground capacitance in neglected in this model. In case of medium and long lines the capacitance and resistance increases. Figure 3.2.-3 represents a long line model and further details about the transmission lines are presented in the Appendix-B.

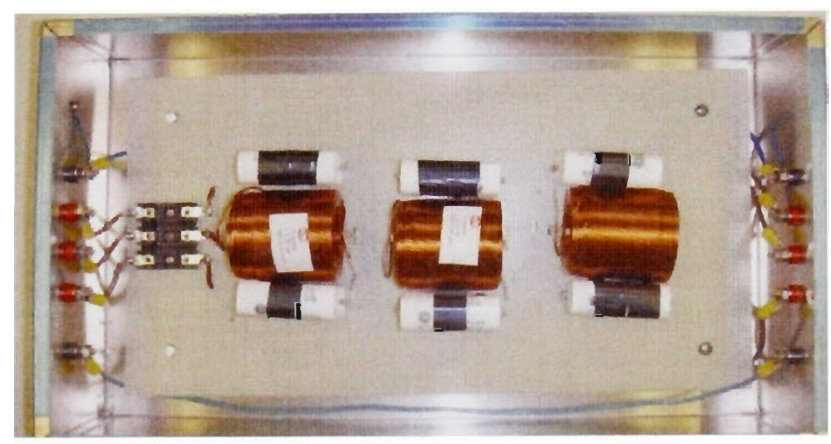

Figure 3.2-3 300 Mile Transmission Line Emulator 


\subsubsection{Transformer based High Voltage Transmission Line}

A transmission line is also developed to emulate a HV line model on the test bed. 6 $1 \mathrm{kVA}$ multi tap single phase transformers with voltage taps of 120,240 and 480 volts are used for this emulation. A simulation of the system is conducted to verify the high voltage line with transformers on the system prior to the implementation for $120 / 240$ Volt system and results are presented below. The figure 3.2-1 presents the Simulink model and figures 3.2-2 and 3.2-3 present the voltage waveforms at primary and secondary terminals of the transmission line with the physical implementation picture in figure $3.2-4$

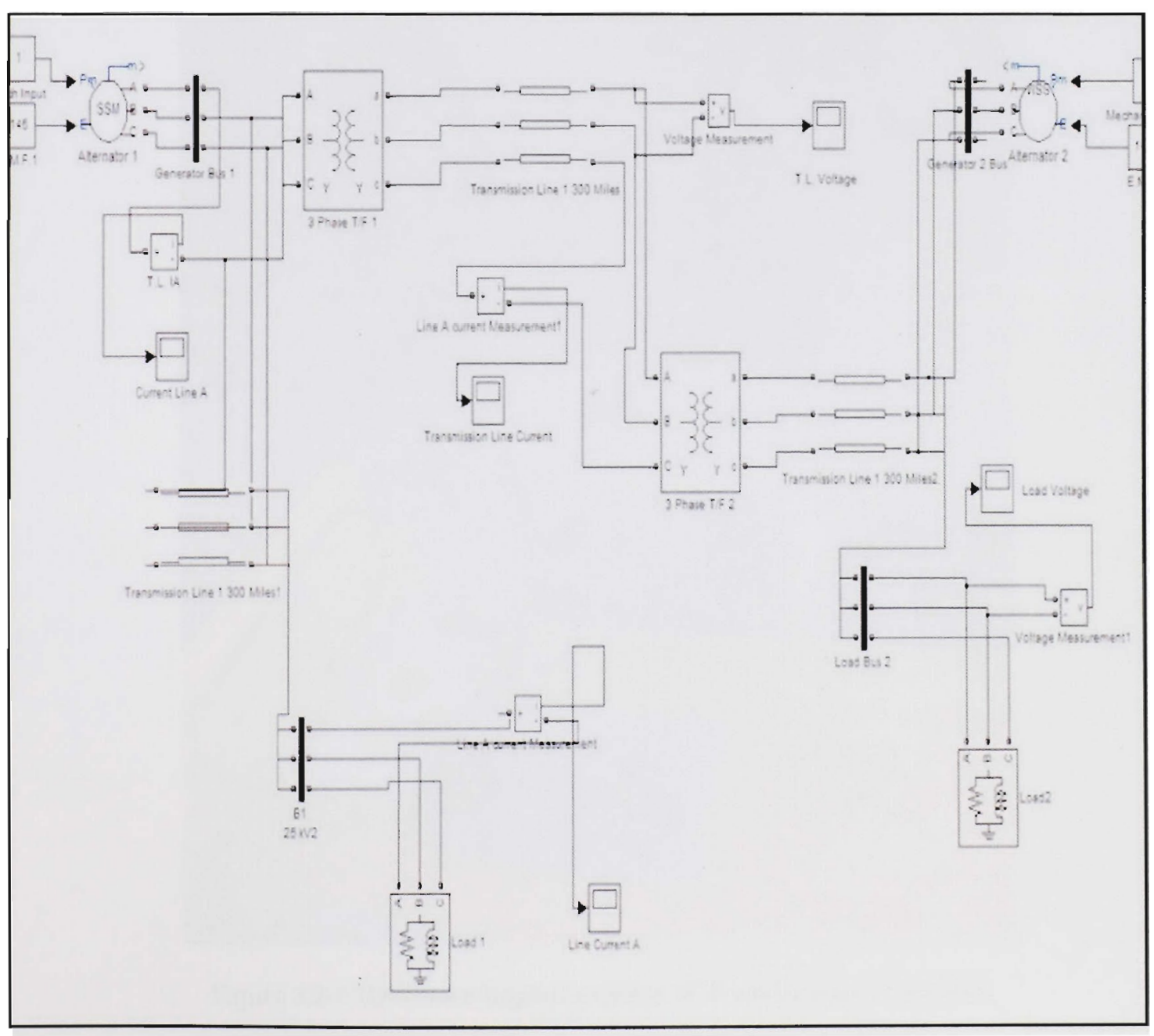


Figure 3.2-1 Simulink model of the partial power system test bed model with transformers based line
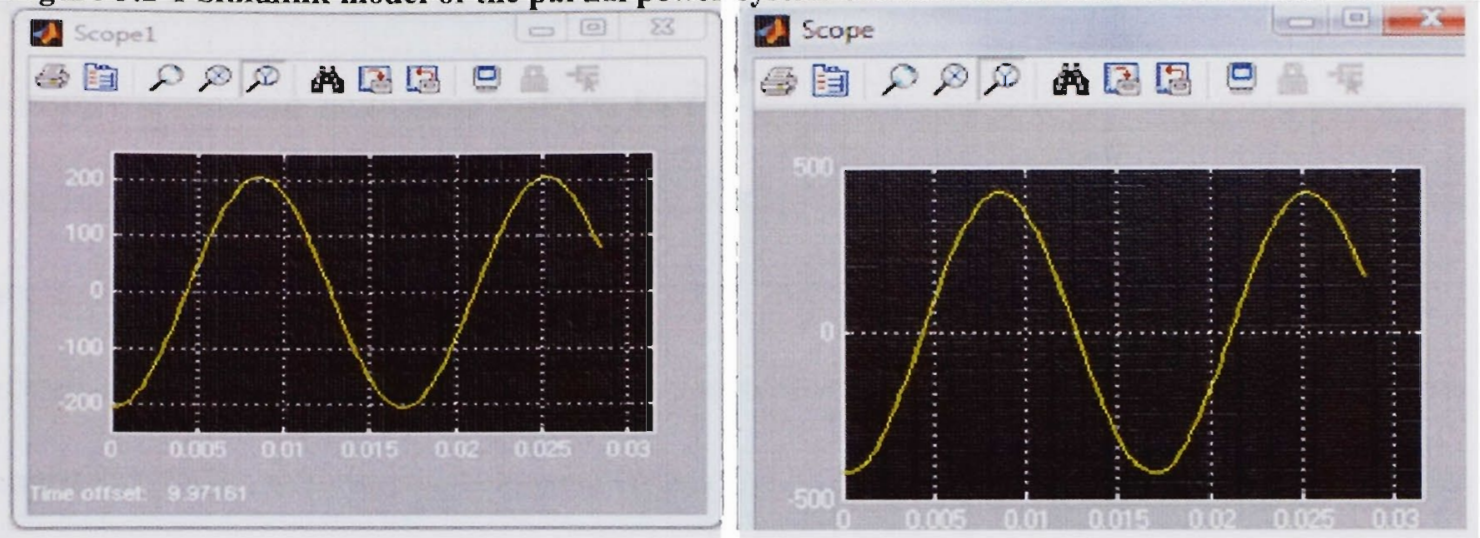

Figure 3.2-2 Primary voltage of the transformer Figure 3.2-3 Secondary voltage of Transformer T.L.

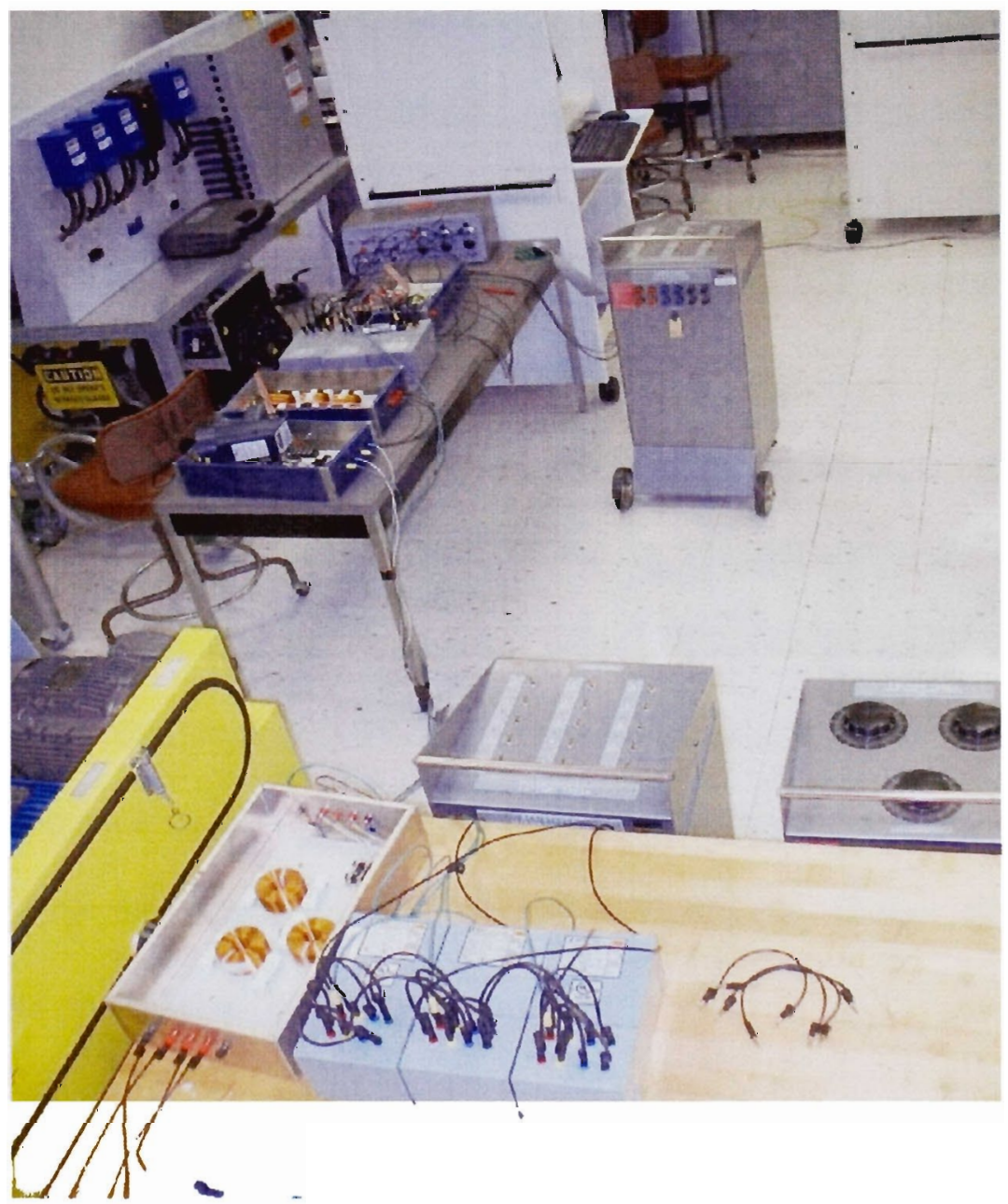

Figure 3.2-4 Hardware Implementation of Transformers based line 


\subsubsection{Generator and Load Buses with DAQ system}

Another important component of the hardware development is the bus bar model. The bus bar model consists of a module that contains switching devices (sensors) and data acquisition terminals. This module is then interfaced to a computer through a data acquisition card from either National Instruments or through dSPACE 1104 control board. The computer uses Labview and Control Desk software packages for data display, assessment, control and data logging acting as a Remote Terminal Unit (RTU).

The bus bar is designed to capture 3-phase current profile and 3-phase line to line voltage profile; this is done through the utilization of voltages and current transformers to step down the voltage to input levels for data acquisition system. The developed bus bar model can monitor the three phase and neutral currents (Ia, Ib, Ic and In) and three phase voltages $(\mathrm{Va}, \mathrm{Vb}$ and $\mathrm{Vc})$, enabling in measurement of frequency, power factor, real power, reactive power and phasor measurements.

Different models of the system are developed based on the input output ratio. 1:3, 3:1, $2: 2,3: 3$ and $1: 1$. Difference in models provides with an advantage of optimizing the bus base on the device of coupling. For example a 1:3 bus helps in analyzing the output of the generator with the measurement devices at output terminal and 3:1 model reduces the measurement components and is customized for measurement on the output side. Figure 3.2-4 shows different models of the bus bar modules and further details of its development are presented in the Appendix-B. 


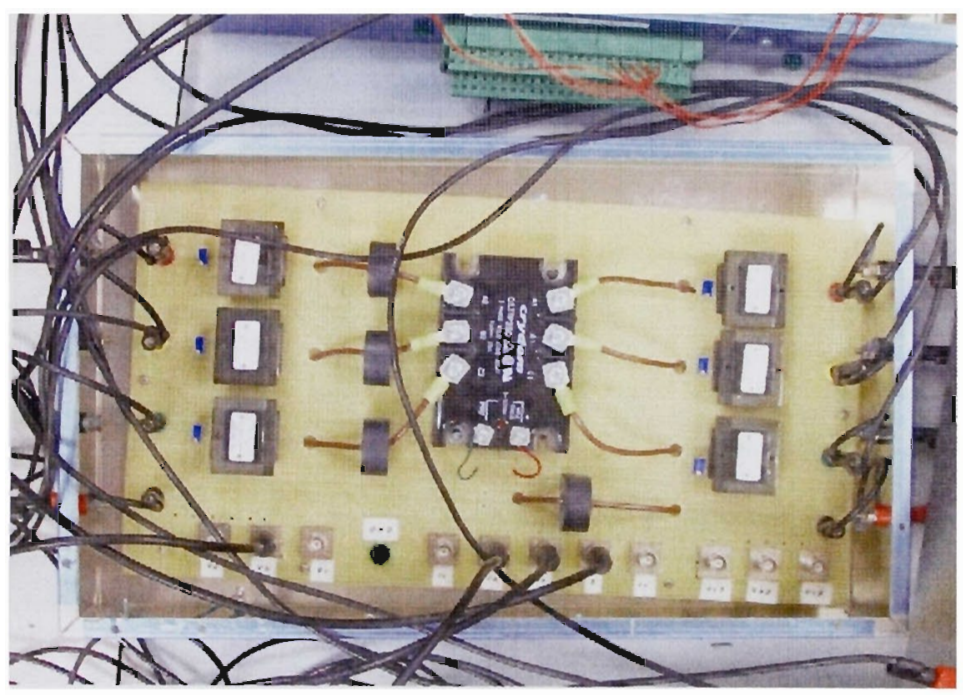

Figure 3.2-5 Load Bus Module with dSPACE DAQ

\subsubsection{Synchronization Module with DAQ system}

An important part of the test bed is the synchronizer module enabling in digital synchronization of different AC generation systems. The module consists of a switch with current and voltage transformers at both the terminals. The measurement outputs are wired to the Labview RTU for control from the Labview Software Package. It has a TURN ON/OFF response time of $1 / 2$ cycle and 100 pin patch output for connection to the Labview PCI slot on the computer. There are two models of the synchronizer module one model is designed to monitor the current and voltage waveforms in three phases at each terminal of the module. Another module is optimized to reduce the components and only uses two VT's on either terminals providing sufficient information for the synchronization experiment. The module is presented in the figure 3.2-5 and further details are presented in the Appendix-B. 


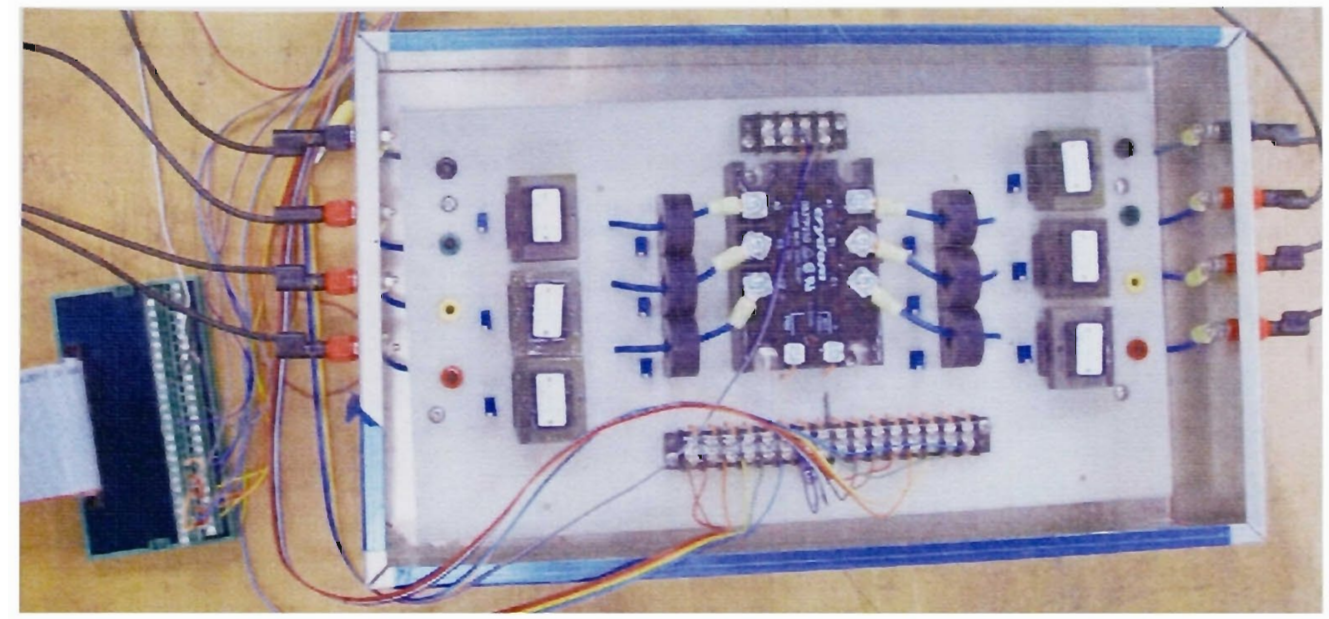

Figure 3.2-6 Synchronizer module with Labview RTL Connector

\subsection{Simulink Model of the Test Bed}

A Simulink model of the power system which was modeled and designed was created to rerify the modeling and stability of the power sy'stem for the application. The modeling values for the Simulink components were provided from the components modeled and designed in hardware such as transmission lines and loads. Figure 3,3-1 presents the Simulink model of the power system test bed.

The model utilized 5 generators and generator buses to represent the real time generating stations and two load buses were utilized to verify the load centers. The base loads modeled for the design of the hardware implementation of the power system test bed were neglected as they do not make any big difference. A subsy'stem was also modeled to emulate a microgrid load center with combustion generator and is displayed in the figure below. 


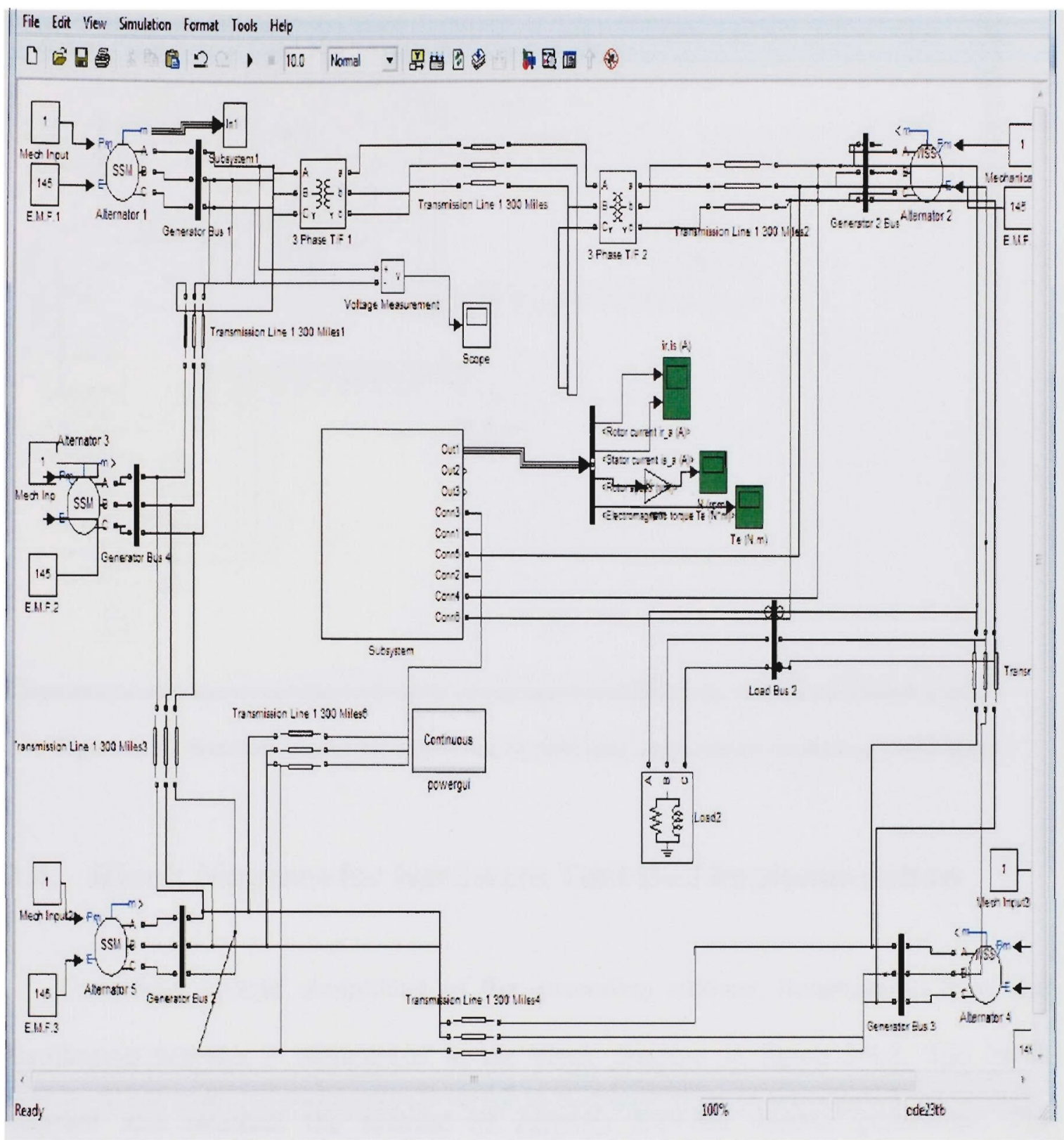

Figure 3.3-1 Simulink model of the power system test bed and microgrid platform 


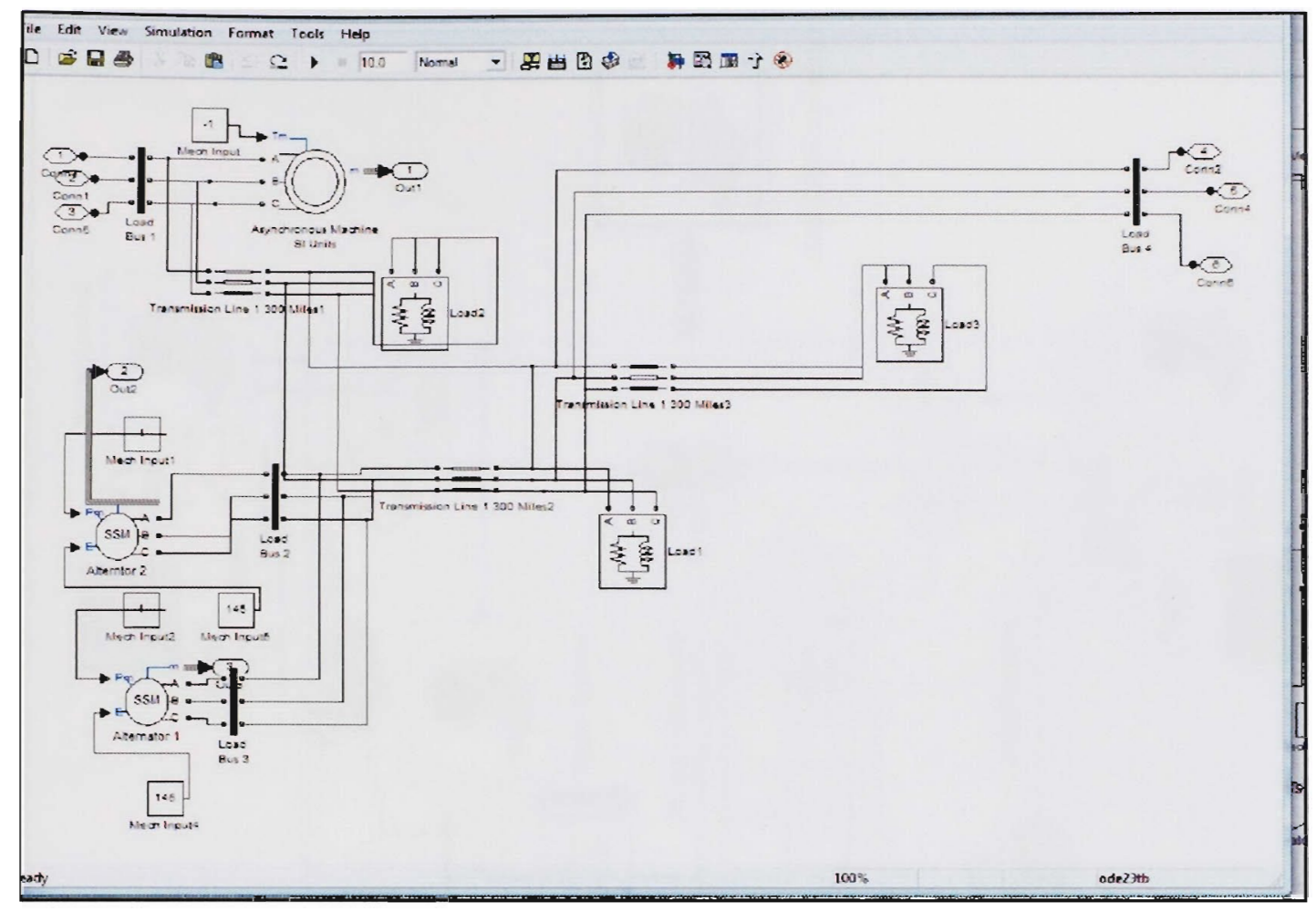

Figure 3.3-2 Simulink model for a load center with micro generation emulating a microgrid

\subsection{Block Diagram for Hardware Test Bed Implementation}

The power system comprising of the generating stations, transmission lines and distribution systems is represented in the block diagram in figure 3.4-1. The block diagram also presents the scheme of network between various generators. The distribution system connecting the loads and the generating stations also is displayed as well. The computers connected to the generating stations represent the SCADA and control systems, the communication network is represented by the dotted lines. The agent control specifies the agent platform installed to allow programming for agent based control system on the framework. 


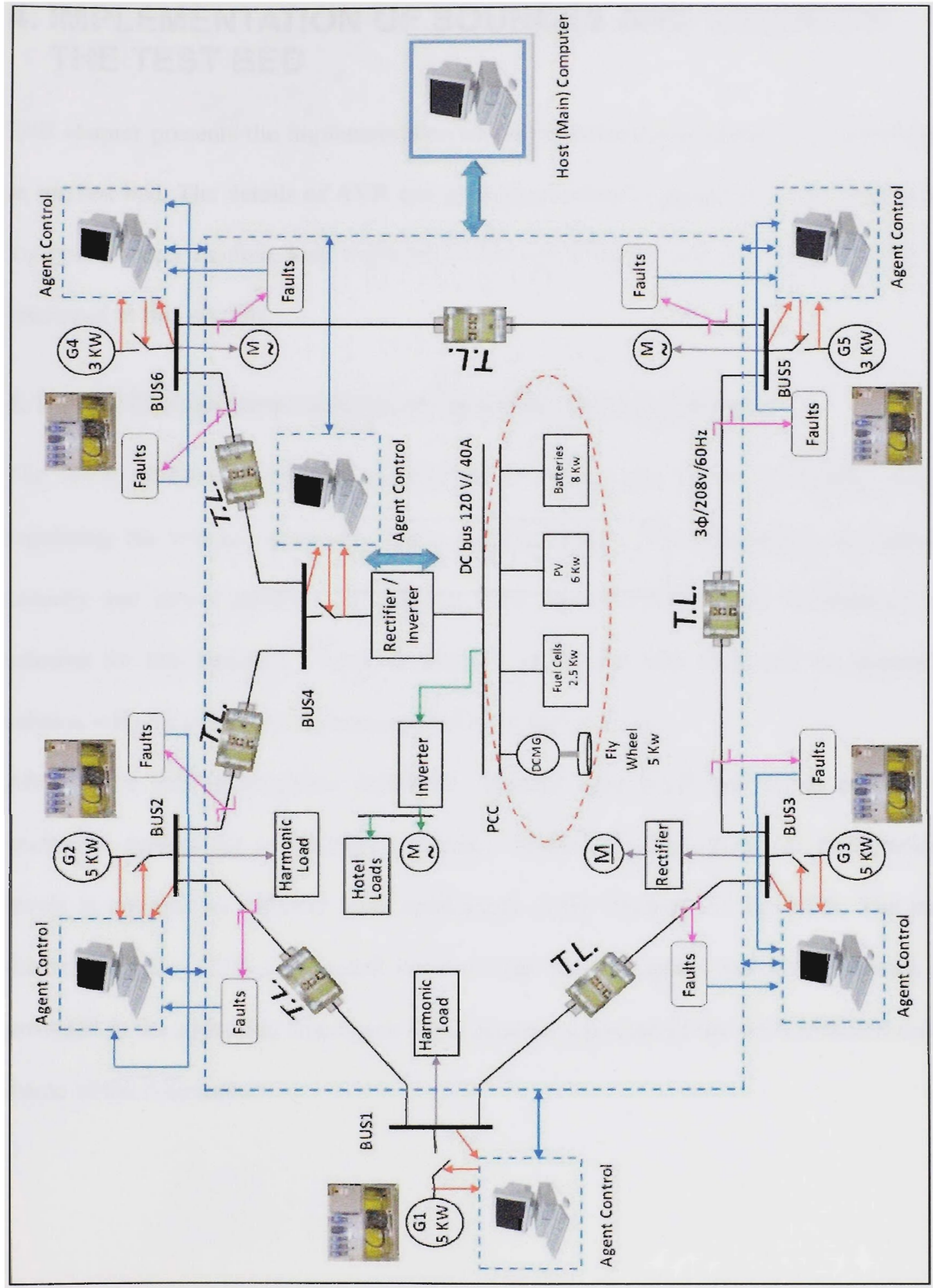

Figure 3.4-1 Block diagram representation of the power system test and microgrid platform 


\section{IMPLEMENTATION OF SOURCES AND LOADS ON THE TEST BED}

This chapter presents the implementation of the developed components to be configured as the test bed. The details of AVR and governor control in generation stations interface for synchronizer module, load implementation and different architectures of test bed are presented in this section.

\subsection{AVR Implementation on the Generating Station}

The AVR (Automatic Voltage Regulator) is a major part of the generating station, regulating the voltage within the limits and constraints. It is responsible for stability, security and power quality. AS 440 an AVR model manufactured by Siemens was selected for this function. A block diagram of the AS 440 AVR and its interaction scheme with the alternator is presented below in figure 4.1-1.

AS440 is a half wave phase controlled thyristor type AVR and forms part of the excitation system for a brushless generator. Positive Voltage build up from residual levels is ensured by efficient semi conductors in the circuitry of the AVR. The main functions of the AVR are divided based on the block diagram and further details are provided in the appendix. The figure 4.1-2 presents a picture of the AVR installed on the frame of the Alternator. 


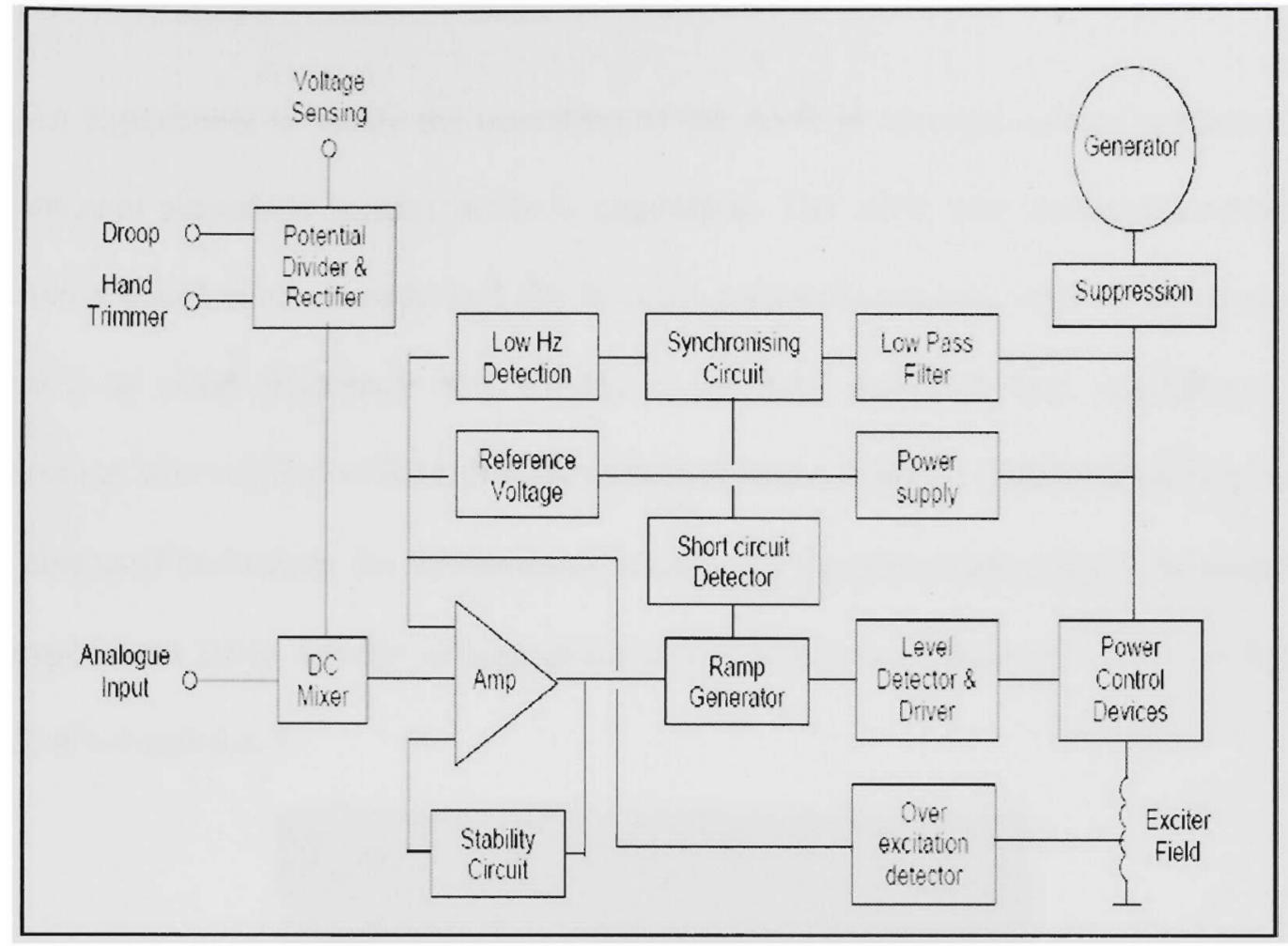

Figure 4.1-1 AVR block diagram displaying the alternator excitation field and the power electronic interface

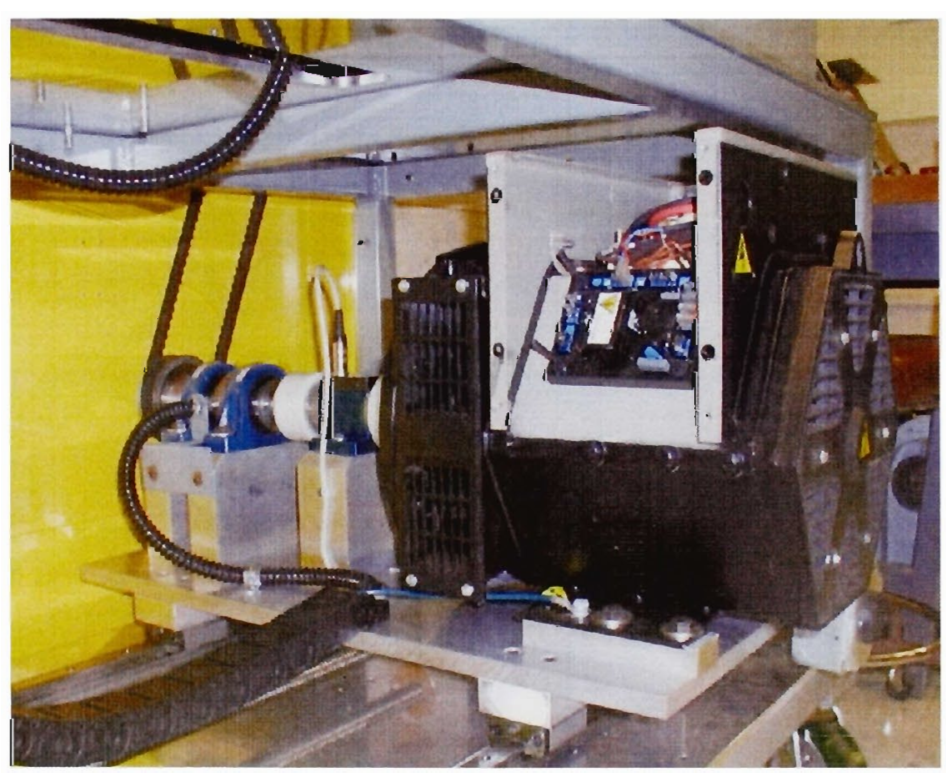

Figure 4.1-2 AVR installed on the front panel of the alternator 
An experiment to verify the operation of the AVR in external manual control mode and internal automatic control mode is conducted. The AVR was operated in both the external manual control mode and the internal automatic control mode. The Alternator operated at rated frequency and voltage at no load and full load conditions. The experiment was verified with both resistive and reactive loads. A transmission line model was also used to dampen the dynamics as in case of a modern power plant. The picture of the experiment setup and the voltage graph of the Alternator output are shown in figures 4.1-3, 4.1-4 and 4.1-5.

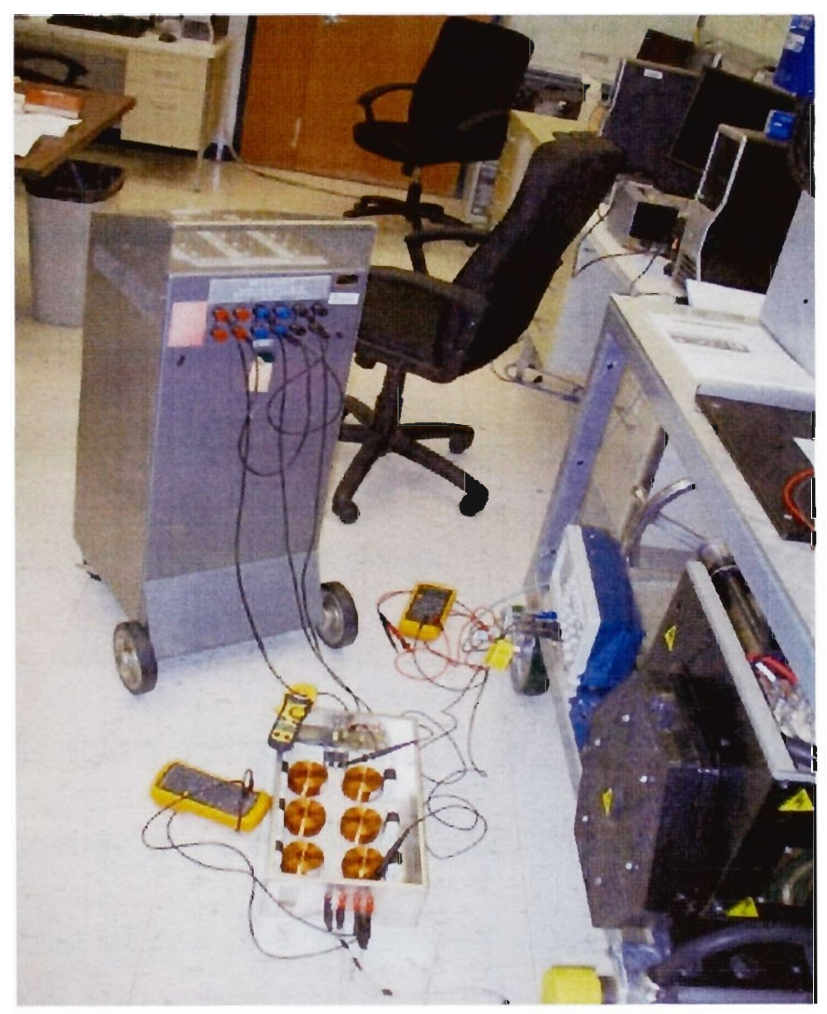

Figure 4.1-3 Experimental setup for AVR and frequency response verification of the alternator 


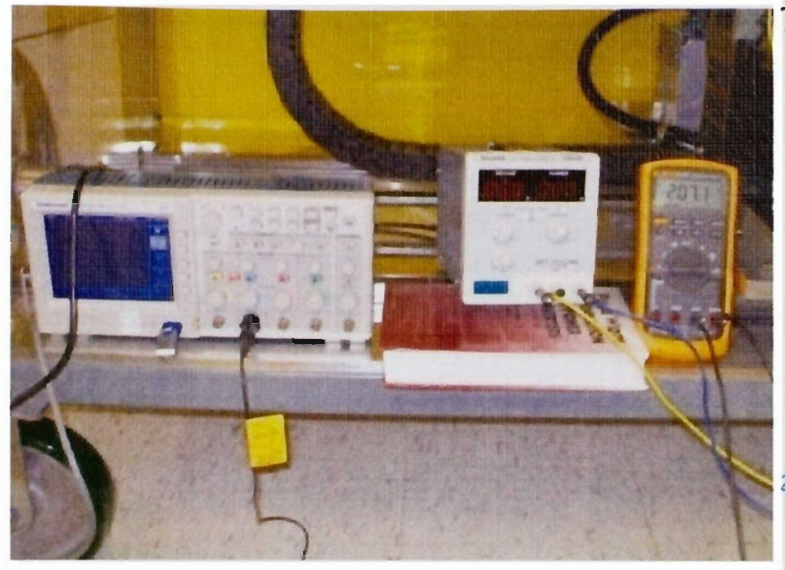

Figure 4.1-4 AVR voltage response

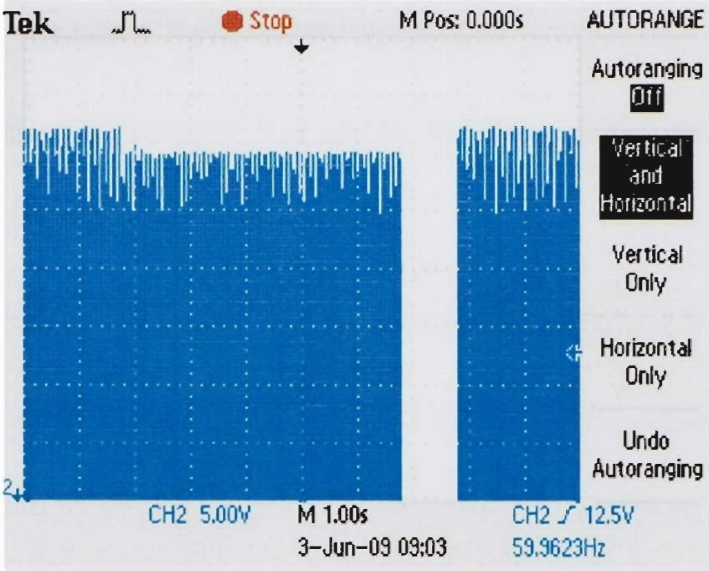

Figure 4.1-5 Alternator response on loading

It was observed that the voltage output of the generating station responded in a better manner with the operation of the AVR in internal automatic control mode than in the external manual control mode. The above figure displays the voltage of the generating station upon loading, the graph displays the damping of the spikes upon loading but it can also be observed that there is no reduction the operating voltage.

\subsection{Frequency Control from Lenze Drive}

The speed control of the prime mover to control the speed of the alternator, eventually controlling the electrical output frequency is called as governor control. It is a critical control for the stable operation of a power system as all the generating stations are synchronized to operate at a constant frequency. Heavy disturbances in frequency can make the system run out of synchronism, leading to tripping of relays and disconnection of generator from the rest of the grid and in worst case, extreme damage to the equipment. 
The prime movers on each of the generating stations have been equipped with Lenze SMV (Space Modulation Vector) open loop drives to control the speed to operation. This emulates the speed control of the governor in a modern power system.

The frequency reference point programmed in the drive will adjust the frequency of electrical power input to the Induction machine acting as prime mover and hence providing a set point to the output speed of the Induction Machine. This system replicates the Steam turbine and governor in a modern thermal power system. The frequency set point to the motor drive of the prime mover can be provided either manually or through a ModBus protocol as the drive is equipped with a communication device working on the ModBus protocol. The control of the frequency can be monitored from the electrical output by the data acquisition system. This control system will then poll a frequency reference set point on the ModBus network, as the ModBus network provides a unique identifier to each machine, the reference point of the instructed machine is changed by the provided value. These motor drives are electronically programmable and can also be programmed for braking and regenerative functions of the machine but this is beyond the scope of this thesis work. The graph in figure below presents the result of the experiment conducted to verify the frequency response of the machine. It can be observed that frequency remains constant at $60 \mathrm{~Hz}$ and also the voltage is not dropping by any considerable value. The experiment set used for verification for AVR response was used for this experiment. 


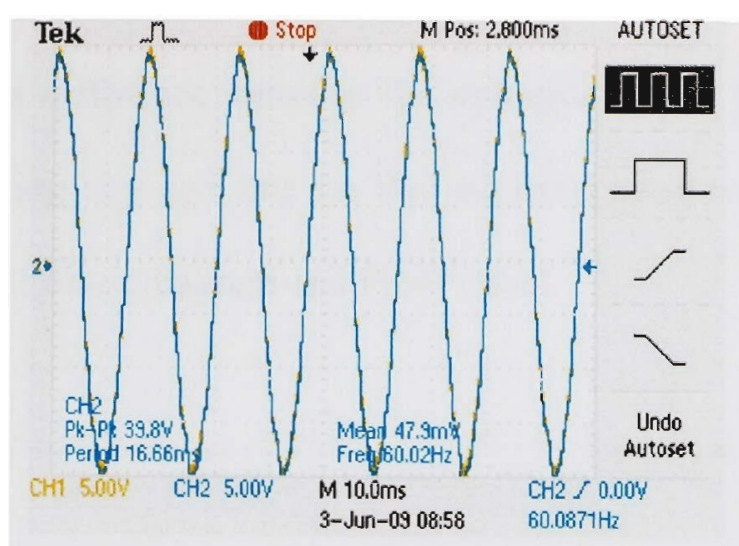

Figure 4.2-1 Frequency response of Alternator on Loading

\subsection{Digital Synchronizer Interface in Labview}

The power system will be implemented by synchronized operation of all the generating stations. A digital synchronizer module was developed based on Labview software and DAQ boards, It obtains the voltage and frequency values from the two generating stations and provides a digital plat form to synchronize the generators on the operating computer. Figure below presents the logic of the synchronizer module.

It can be observed from the block diagram in figure 4.3-1 that the DAQ board acquires the voltage from the generating stations on the left and right of the synchronizer modules. The Root Mean Square (RMS) value of the voltage magnitude and the frequency are then calculated by the logic implemented in the software. The software module acquires voltages of two lines from both sides of the synchronizer bus. The operator can synchronize the system by clicking the synchronize check button by verifying the voltage magnitude, frequency and phase angle. The phase sequence of the lines on both sides of the bus can be verified by checking if voltage of both the lines displayed in two different graphs peaks at the same time. If the lines are not in same phase sequence then there will 
always be a 30 degree difference between the voltages of the two lines, this can be adjusted by interchanging any one line on the incoming generator side. Figure below presents the Labview VI block diagram and Front Panel.

\section{Main Grid Foltage and Frequency}

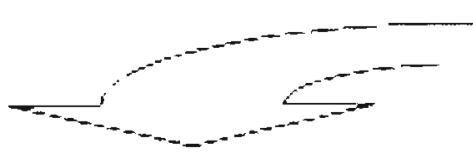

Labview DAQ NI 6025E/Labview Software verifies and monitors both sides of the Bus and implements the decision.
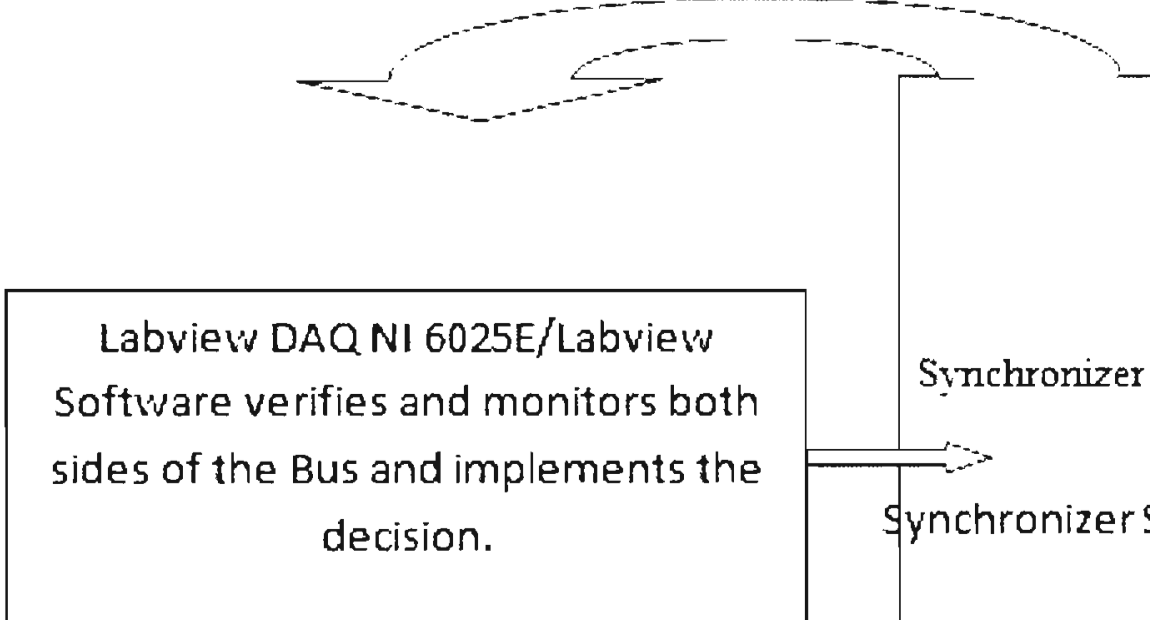

Synchronizer Bus

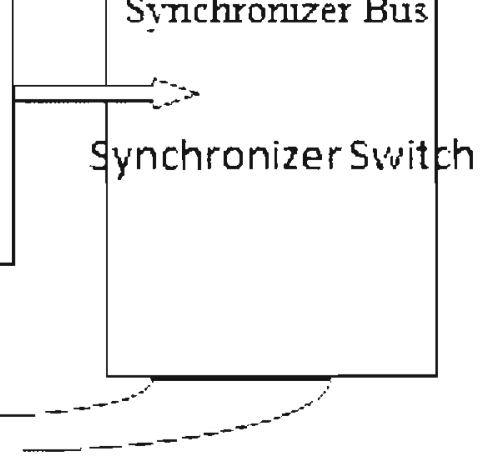

\section{Incoming Gen Yoltage and Frequency}

Figure 4.3-1 Synchronizing Module operation 


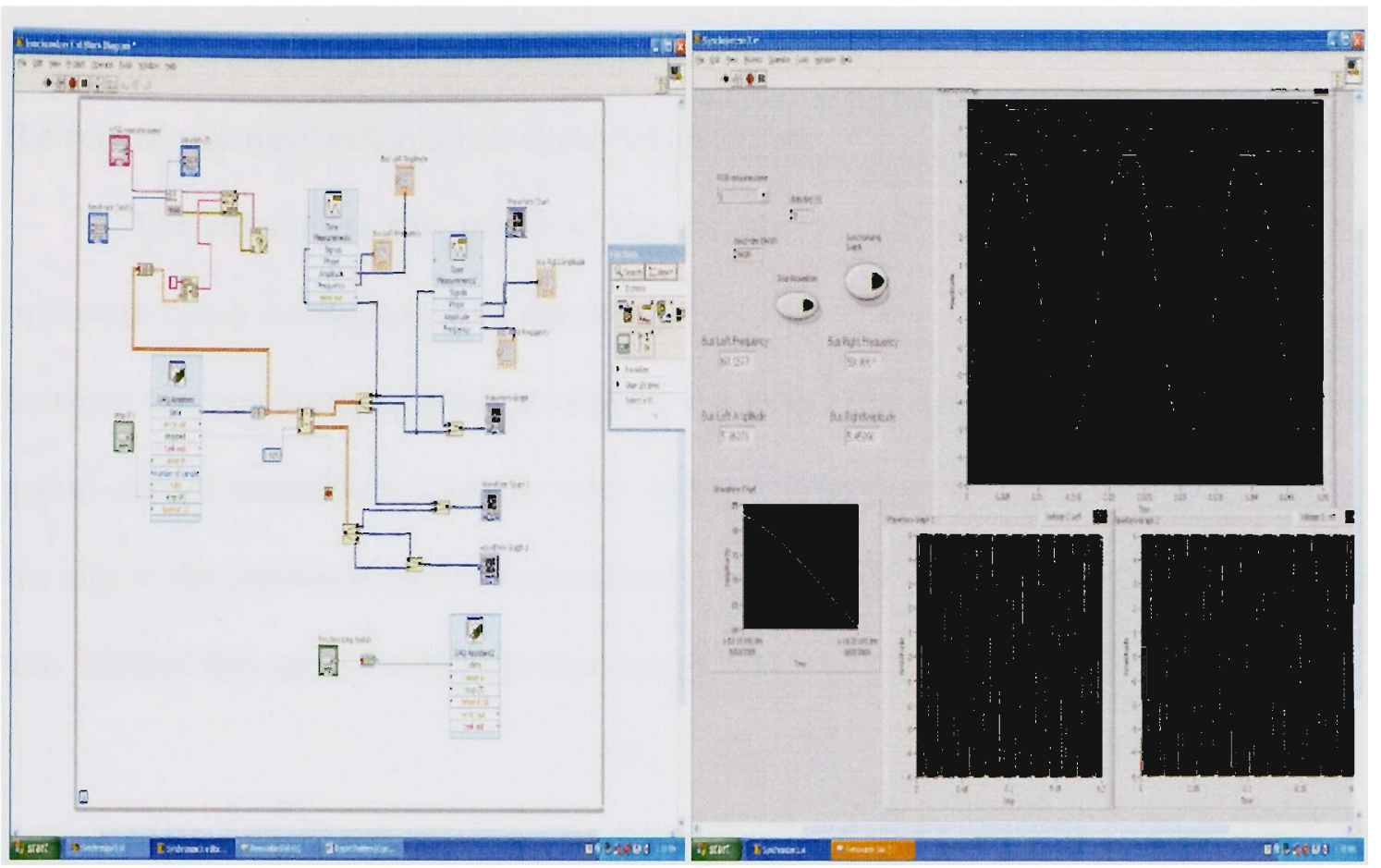

Figure 4.3-2 Synchronizing Module, Labview V.I. Block Diagram and Front Panel

A major hurdle in the synchronization of the Alternators was due to the Induction motor being utilized as a prime mover. Frequency of operation of a power system is $60 \mathrm{~Hz}$ in the United States and a variation on either side of the scale up to $0.02 \mathrm{~Hz}$ is allowed. In case of synchronization in the power system, problem was arisen due to the speed of two induction motors not being the same. To understand the problem, consider two generating stations about to be synchronized. Frequency of the first generator being $60.003 \mathrm{~Hz}$ and the second generator being $60.004 \mathrm{~Hz}$, although the frequency of operation is within the limits for power system stability, the machine drives detected an error. The motor drives are supposed to operate at the programmed frequency, but as the generating stations were synchronized the speed of operation was same for both the generating stations and the prime movers. Due to this the prime mover of the machine operating at $60.003 \mathrm{~Hz}$ started rotating at $60.004 \mathrm{~Hz}$, which is faster than the programmed frequency 
making the Induction machine work as a Induction generator. This resulted in tripping of the second machine within a few cycles of operation.

The solution to this problem was attained by manipulation of induction motor operation based on the operation characteristics. Slip of the induction motor is difference between the synchronous speed created by the magnetic field and the speed of the rotor and in case of mechanical load, the rotor starts receding and the slips starts increasing. As the slip of the Induction motor as presented in the equation below depends on the load, It was decided that upon loading the Generating

$$
s *(N s-1)=N r
$$

Station before synchronization, the machine frequency reference will be higher than the operating frequency to account for frequency fall due to load and the load is exactly equivalent to the base load on the power system.

\subsection{Load Connection and other System Integration}

There are two different types of loads modeled for implementation on the test bed as constant impedance and constant current models. The constant impedance model is implemented by using resistance and inductance boxes. The resistance-reactance load device has three variables, resistance, capacitance and inductance. The value of the resistance in controlled by toggle switches provided on the interface panel. The reactance load is controlled related knobs which serve to adjust power factor of the load for either lagging or leading power factor. Another model of constant impedance load is based on lighting load emulator controlled through the Labview DAQ device. It can be varied to 
draw up to $1 \mathrm{kVA}$ of power and can be programmed as cyclic variation through Labview RTU.

Constant current load model is implemented to induction motor drives connected to bus bars on the test bed. The bus bar can be controlled from dSPACE or Labview computer and the load on the motor is adjusted through a mechanical brake applied on the shaft of the motor.

\subsection{Architectures for Test Bed Configuration}

The test bed is implemented in a manner where it can be manipulated and changed to emulate various power system configurations. As the test bed is supposed to model terrestrial and mobile power system the components have been developed in a manner to provide a flexibility to vary the configuration of the test bed.

It can be connected in a serial bus, ring and other architectures for the required experiment. As the test bed is to be integrated with distributed resources and microgrids the test bed has a capability at various points to integrate with other systems. The different modes in which the test bed has been connected and operated are presented in the figures 4.5-1, 4.5-2, 4.5-3 and 4.5-4. The hardware implementation picture of the test bed is presented in figure 4.5-5. 


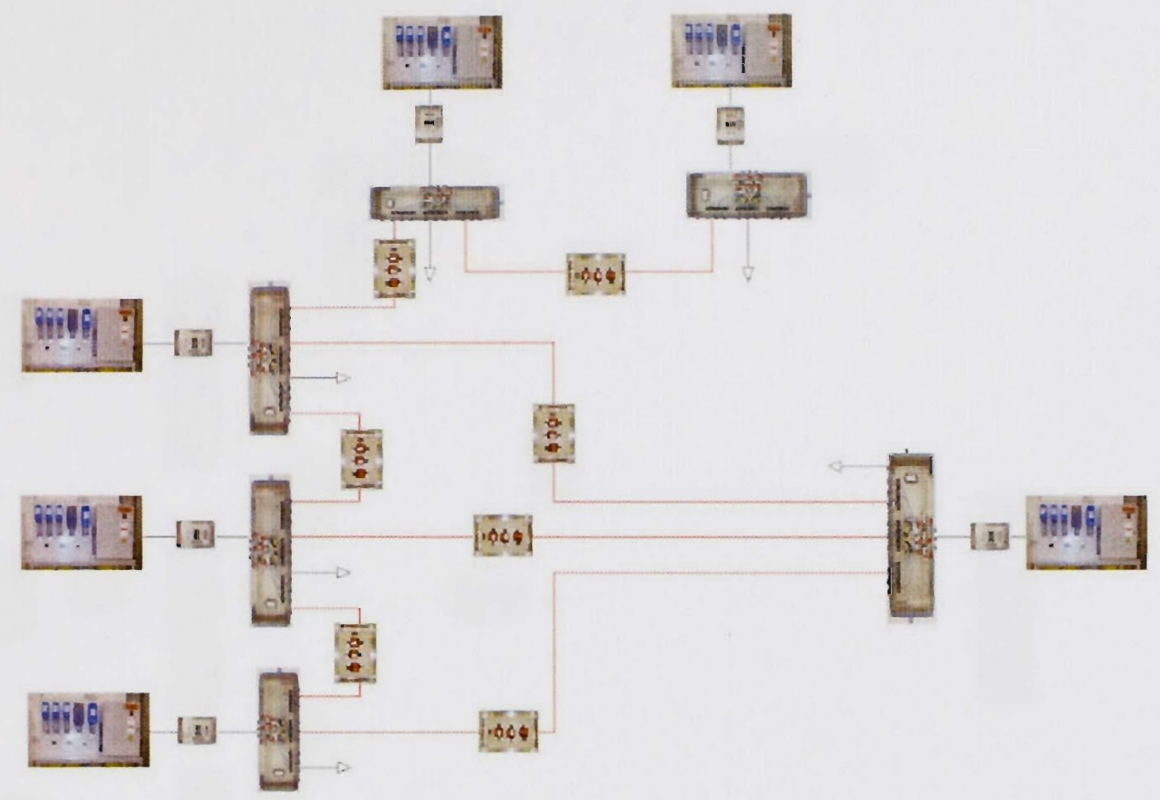

Figure 4.5-1Two Tier Zonal Architecture connection of the test bed

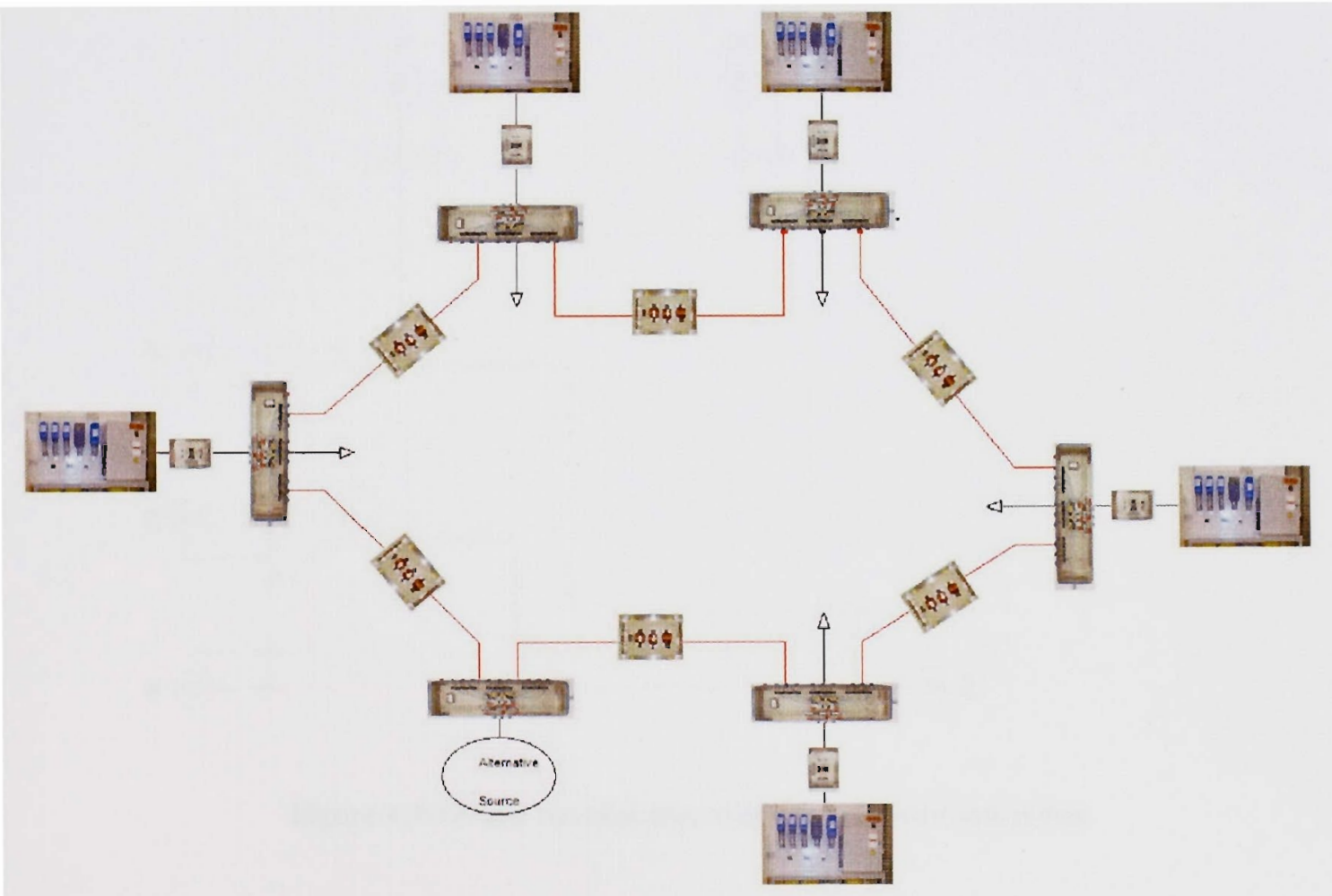

Figure 4.5-2 Ring Bus Architecture of the test bed 


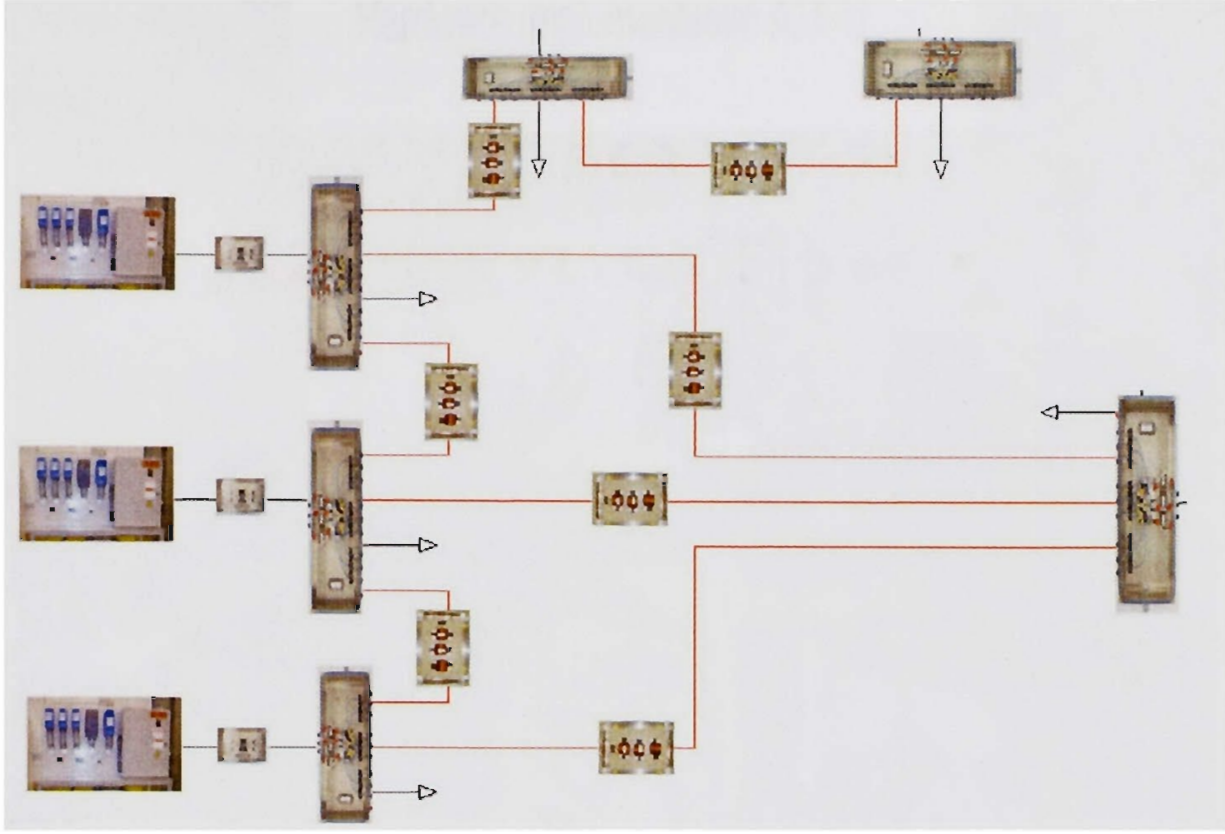

Figure 4.5-3 Serial Architecture with Three generator Test bed

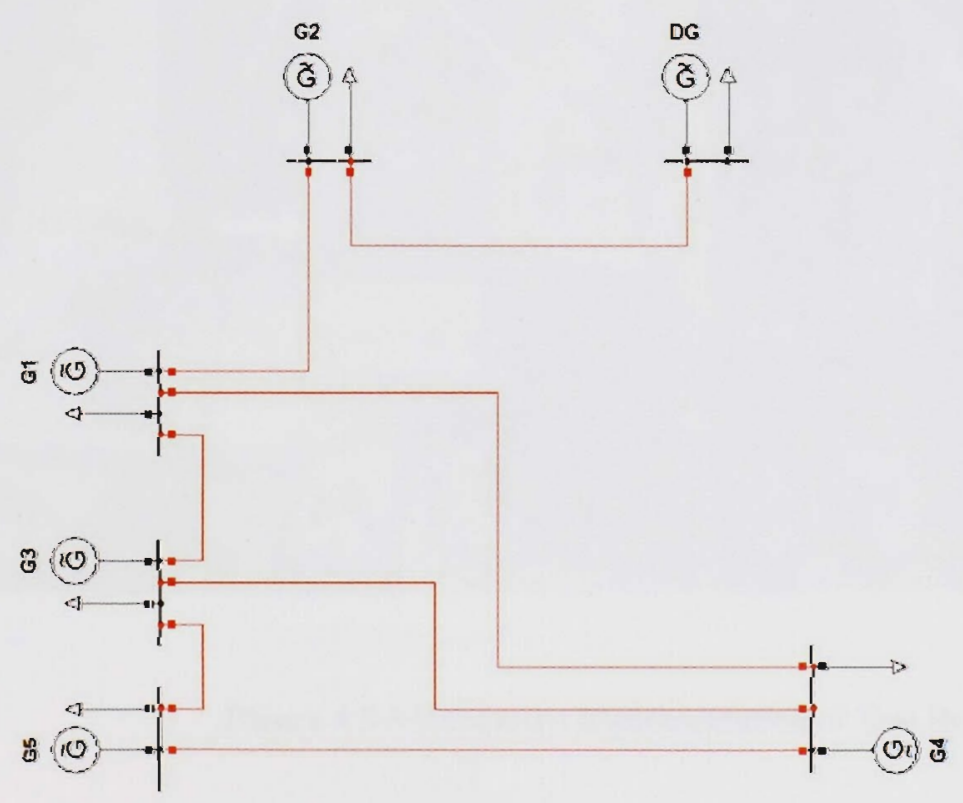

Figure 4.5-4Zonal Architecture with Distributed Generation 


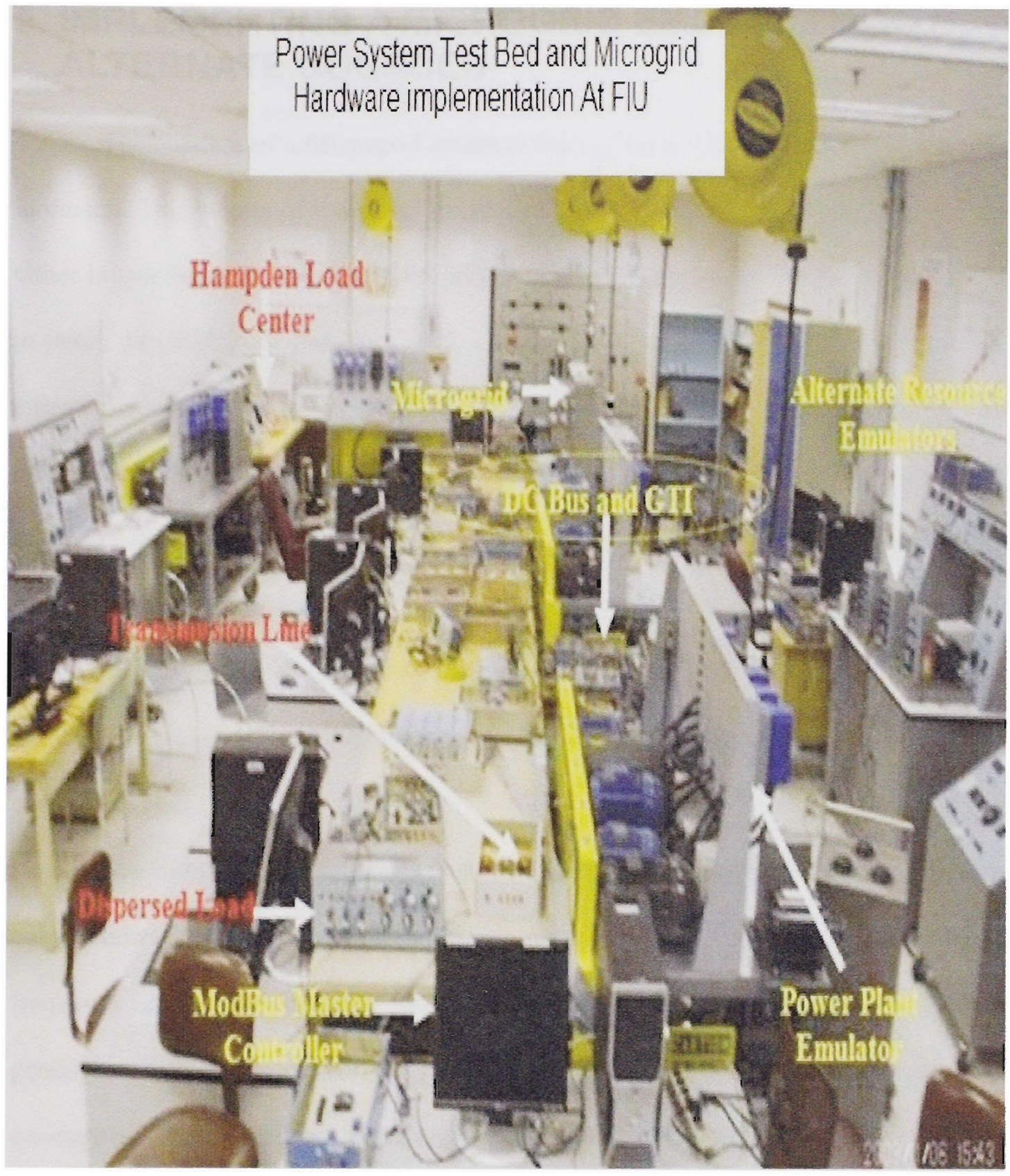

Figure 4.5-5 Hardware Implementation of Test Bed 


\section{IMPLEMENTING A MICROGRID EMULATOR WITH ALTERNATE SOURCES}

The concept of a Microgrid assumes that a cluster of loads and generators operate in unison. This cluster of loads and generators based on the state of operation can operate either in synchronism with the main grid or function alone as an individual power system in itself, isolated from the main grid. The idea behind this is to utilize the power and energy resources which are available within the load area and compensate the power drawn from the grid in case of synchronized operation. In addition to this during any faults on the main grid, the microgrid can disconnect itself from the main grid and provide an uninterruptible power supply to the internal loads increasing the reliability. As the local generation system forms a cluster with the loads, this generally can happen only at the load centers, geographically representing a town, village, county and in some cases even a city [17].

The sources are generally renewable but large microgrids can also employ combustion generators and other unconventional sources to support the voltage and frequency during the islanded mode of operation. The sources accounting for local generation in a microgrid are PV Array, fuel cells, wind Generators, small gas turbine generators, microturbines, batteries, ultra capacitors and fly-wheels. Combustion generators, microturbines and gas turbine generators are dispatchable sources among the above, whereas wind generators and PV arrays are intermittent, with the rest being storage resources [18-19]. 
There are many topologies in which the internal generators, loads and storage systems can be interconnected and controlled to form a microgrid. As this concept is relatively young new concepts might come into existence when this thesis is being written. We discuss some popular and effective topologies which are either deployed or have been considered technically superior.

One of the methods is to rectify the output of the non-conventional $\mathrm{AC}$ resources and connect all the generators to a common DC bus, which is later connected to a power electronic inverter which regulates the $\mathrm{AC}$ voltage and frequency. The output of the $\mathrm{AC}$ bus is then connected to the Load point and also to the main grid. This is called as the point of common coupling. The interconnection between the main grid and the microgrid at this point is based on a certain logic implemented either by the microcontroller of the power electronic inverter or an Agent. The storage system is connected at the DC bus, the connection is made in such a way that the storage system charges when the power is excessive or during the normal operation and discharges during the power shortage. This operation is again based on the logic implemented. In this topology the loads are divided into two types, critical and normal. In case of normal operation when the power is able to meet the demands both the loads are supplied power but in case of shortage in power availability critical loads are given priority and hence load shedding is initiated with normal loads. The loads are assumed to be controlled loads.

In another topology, if the power generated through the source provides a DC or varying output then it is rectified in the first stage, then these resources can be connected either to a same DC bus or different buses based on the voltage. This is later connected to an Inverter which regulates the $\mathrm{AC}$ output at a constant voltage and frequency of 
an Inverter which regulates the $\mathrm{AC}$ output at a constant voltage and frequency of operation, the $\mathrm{AC}$ power from the dispatchable resources is then synchronized at $\mathrm{AC}$ bus, which is connected to the load and main grid at the point of common coupling (PCC). There is also a small variation of this topology, in case of geographically large microgrids all the resources might not be available at same point, hence the resources are directly connected to the loads and the synchronization of all the resources might occur along the distribution system. This method does not consider a separate interconnection system for the resources as in the earlier topologies and is more practical.

This thesis aims to implement the concept of distributed generation not only on terrestrial systems but also on shipboard and other mobile power systems. In shipboard and other mobile power systems there are minor differences in consideration with grounding, load management and critical applications, we neglect these minor differences as this work aims at developing an approximate power system platform for all power systems research. The concept of Microgrid varies between terrestrial power systems and shipboard power systems. Mobile power systems do not have separate load centers which demand continuous power supply and have different interests than the main grid. In fact they have only one grid; however the loads are different with critical loads, non-critical loads, continuous loads and intermittent loads. The logic here is to supply power based on the circumstances, this is generally implemented by the control system. The aim of these power systems is to extract maximum power from the non-conventional sources and optimize the operation of conventional sources. At the same time there is no isolated mode of operation. The system should always supply power based on the priority of control. The AC operation of these power systems generally occurs at higher frequencies 
and higher voltages, present trend is also to use DC for loads. Hence, all these resources are connected in a ring network and the loads are connected through rectifiers. The nonconventional generation resources are connected through Inverters, the resources capable of utilization in these kinds of system include PV array, Fuel Cell and tidal energy. Combustion generators, oil and gas turbines are the conventional sources for these power systems.

\subsection{Microgrid Operation}

\subsubsection{Grid Connected Mode of Operation}

This is the synchronized operation of the Microgrid. In this mode of the microgrid, the controller at the point of common coupling or the inverter takes the reference for the Voltage and frequency of the microgrid from the main grid. In this operation the microgrid is unable to generate power required for the internal loads, hence it draws power from the main grid. Main grid is responsible for voltage and frequency support to the microgrid and also to supply active and reactive power as presented in figure 5.1-1.

In this mode of operation if the resources in the microgrid generate more power than the internal loads require. The main grid can draw power from the microgrid, according to the NERC regulations the main grid should possess a capability to draw the power and for the microgrid to maintain the power at certain standards. However, the main grid can select to purchase power from the microgrid or from another microgrid or generate its own power based on the economic market and main grid load conditions. In 
case of main grid rejecting the power from the microgrid, the excess power is diverted either to storage or to dump loads.

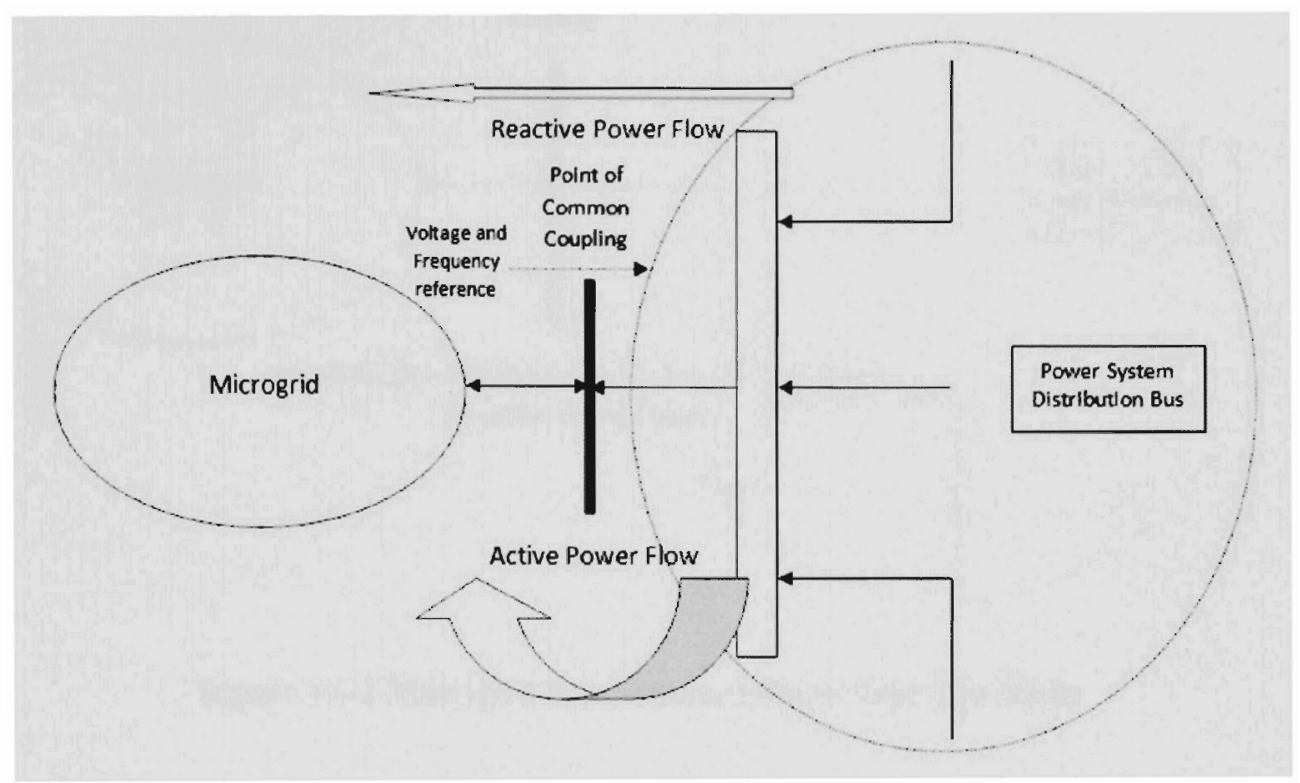

Figure 5.1-1 Microgrid Synchronized Power Consumption Mode

As this also is a synchronized mode of operation the main grid provides the reference for voltage and frequency. Although the microgrid is injecting the power into the main grid, the main grid is supposed to support the voltage, frequency and supply the reactive power as well to the microgrid, figure 5.1-2 displays the directions of active and reactive power flow. 


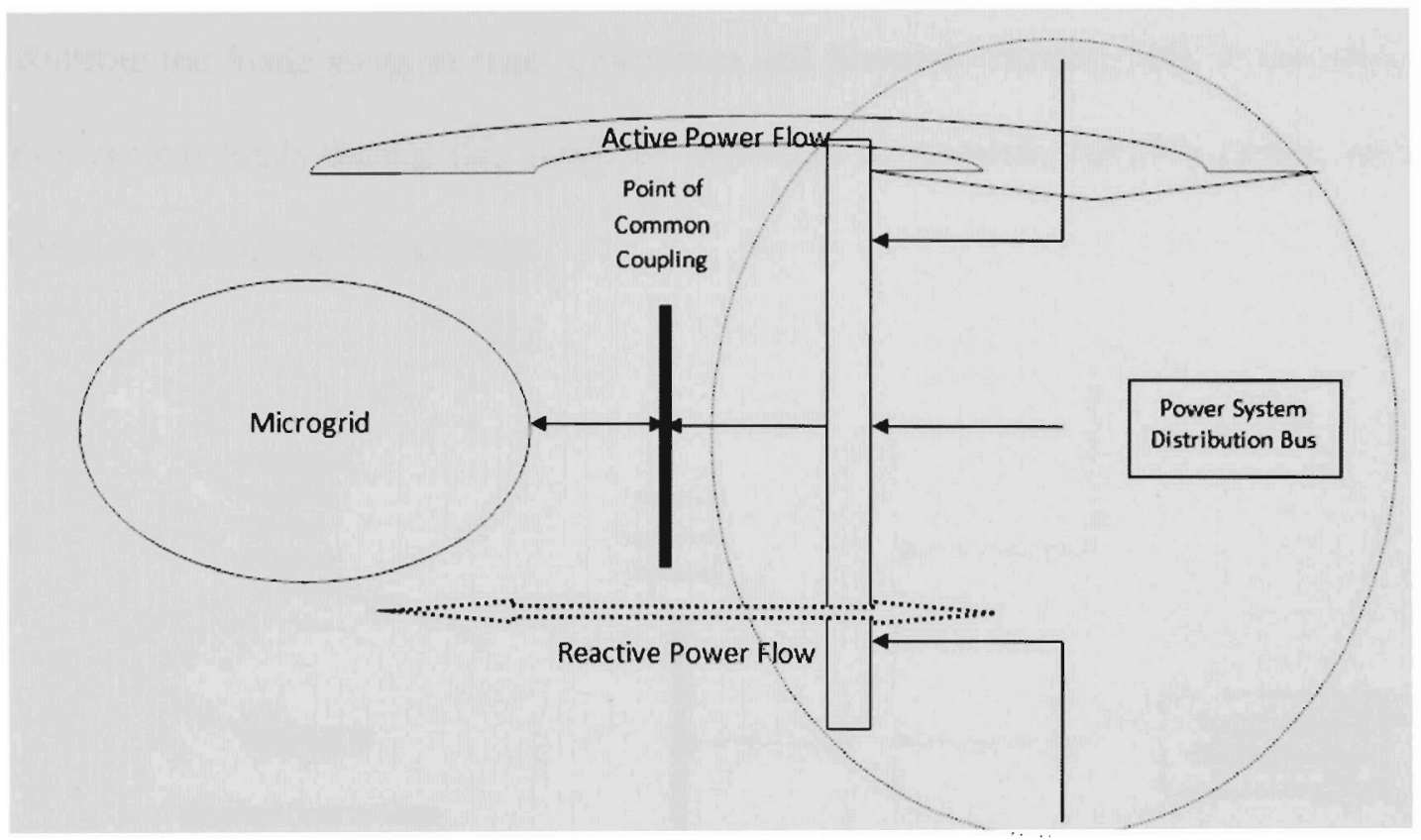

Figure 5.1-2 Microgrid Synchronized Power Injection Mode

\subsubsection{Islanded Operation}

In this mode of operation, the microgrid and the main grid disconnect. This might happen due to two reasons, low power quality or a fault within microgrid, secondly it can also happen due to low power quality, fault or a black out on the main grid. Generally in synchronized mode if the power quality of the microgrid is low the main grid will not accept power from the microgrid but will still maintain synchronization as the voltage and frequency are supported by main grid. However in case of a fault in the microgrid, the main grid will disconnect itself from the microgrid. In second case when the power quality of main grid is low or during a fault on the main grid, the microgrid isolates itself from the main grid. This is called as Islanded mode of operation. In this mode the controller takes the reference of frequency and voltage from the programmed constant values. The control system manages the loads by verifying the voltage and frequency. It 
controls the loads so as to meet generation and demand criterion [20]. It can also shed non-critical loads during this mode of operation to maintain reactive power, so as to maintain voltage and frequency.

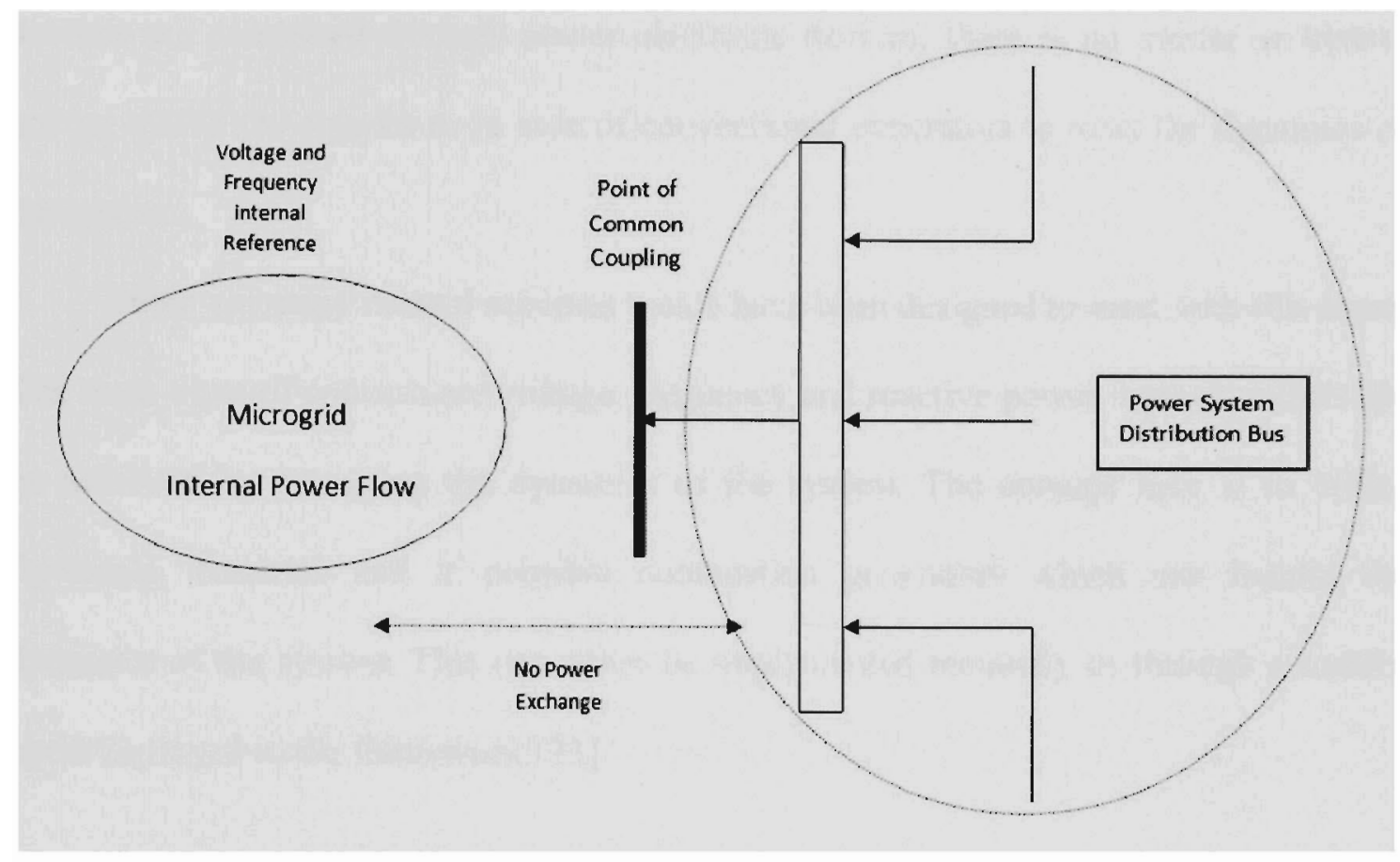

Figure 5.1-3 Microgrid Islanded Mode of Operation

\subsection{Black Start Issue}

The restoration of power after complete outage is called as Black Start. In a conventional power system restoration is a very complicated process, involving manually carried tasks according to predefined guidelines. They have to be completed in a fast way, in real time and under extremely stressed conditions. These complexities make the decision support tools to assist system operators extremely valuable.

However, this process is different in case of a microgrid as the independent variables affecting the system are lower in number compared to the conventional power system. Although this reduces the complication, the incompatibility of microsources to be 
directly connected to the loads creates a new problem. The characteristics of these sources are not viable for direct connection to the $\mathrm{LV}$ grid as the voltage and frequency fluctuates and also there is no support for the transient reactive power, as most of these sources are connected through power electronic devices, there is no inertia or kinetic energy within the sources as in case of conventional generators to meet the dynamics of the system.

There are many control schemes which have been designed to meet with this issue. The main areas of concern are voltage, frequency and reactive power support, which can be achieved by managing the dynamics of the system. The concept here is to utilize flywheels, batteries and if possible combustion generators which can handle the dynamics of the system. This can either be implemented manually or through a control agent deployed in the framework [21].

\subsection{Microgrid Model Implemented on the Test Bed}

Microgrid model on implemented on the test bed emulates PV array, wind energy generator, combustion generator and battery array for storage. The microgrid is modeled as a load center with independent generation through alternate resources [23]. The alternate resources are connected to DC bus level distribution system. DC voltage is maintained same at one bus but is controlled and variable at different levels on different buses for connecting different loads on the DC system. Figure 5.3-1 represents the implementation of the microgrid on the test bed as a block diagram and figure 5.3-2 presents the hardware implementation. 


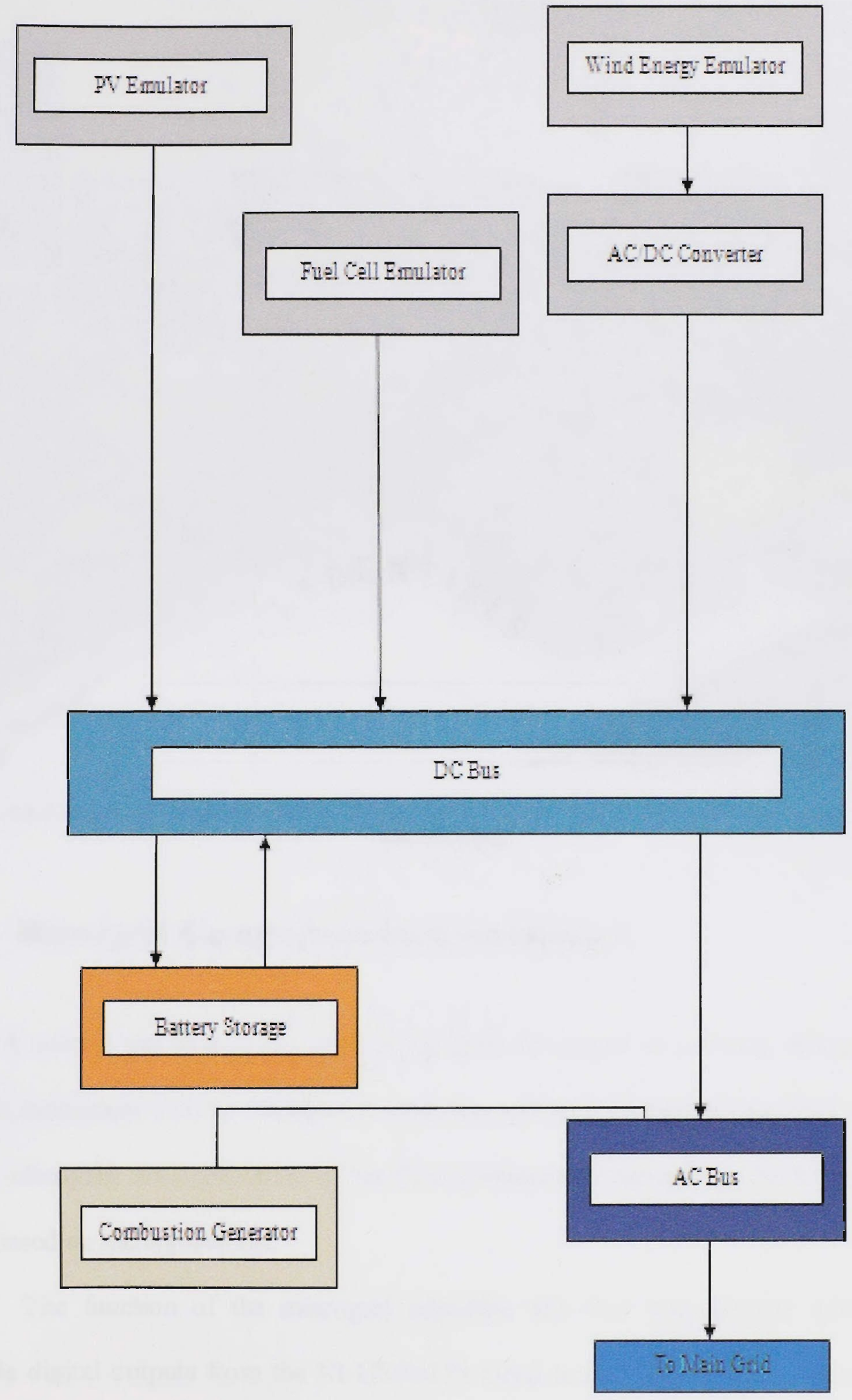

Figure 5.3-1 Block Diagram of the Microgrid Model Implemented on the Test Bed 


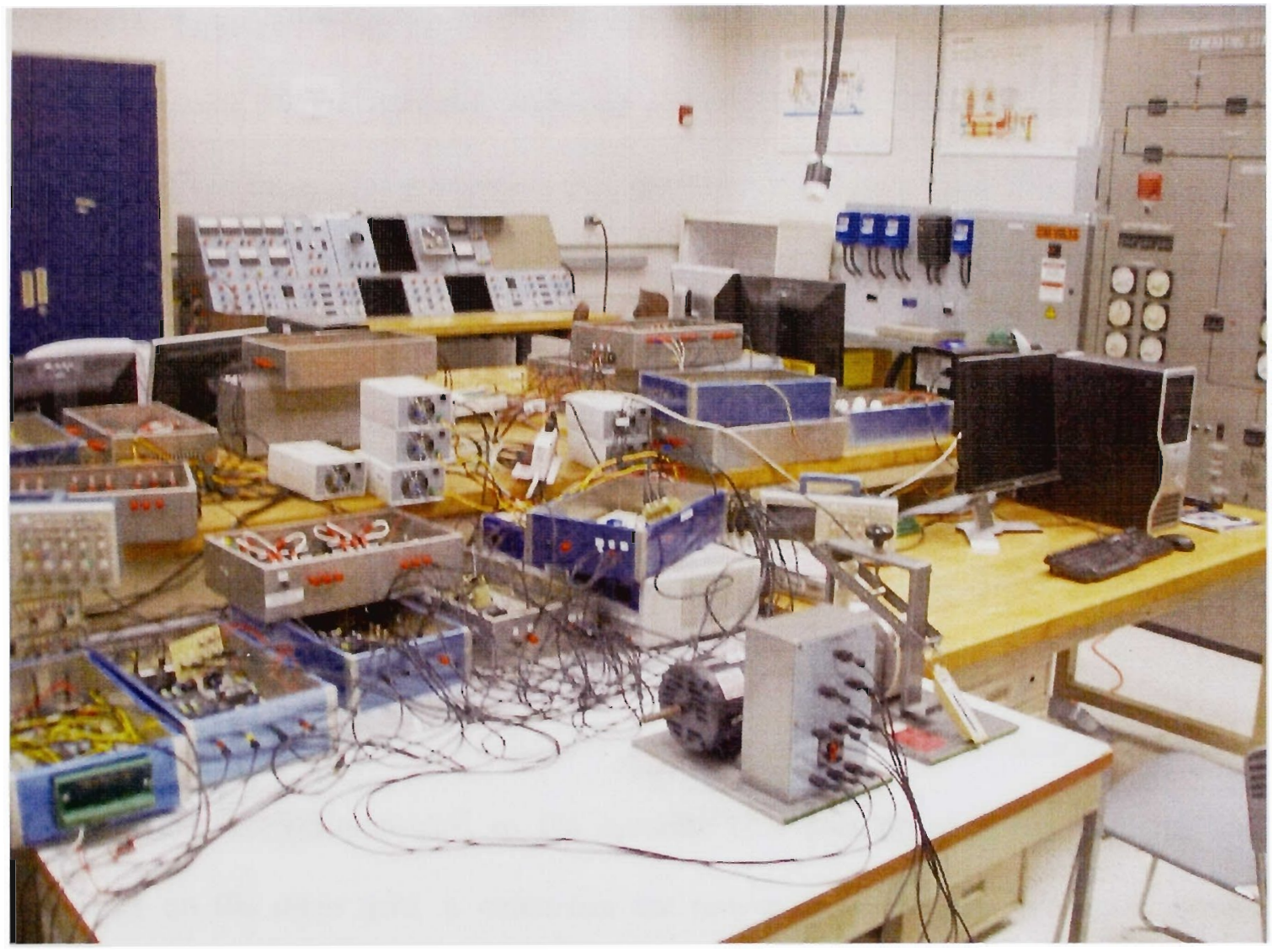

Figure 5.3-2 Hardware Implementation of Microgrid with DC Bus, Loads and Monitoring Station on the Test Bed

\subsection{Microgrid Control Interface in Labview}

A control and monitoring module has been developed in Labview. It's a Labview Virtual Instrument (VI) to manage the islanding and grid connected operation and loads in the microgrid. An explanation of the VI is presented by decimating the VI in different parts based on the application.

The function of the microgrid islanding and load management system is to provide digital outputs from the NI USB6259 DAQ board. The top two outputs on the front panel are programmed for the two digital output channels in the DAQ assistant 
toolbox in Labview. They have been connected in not gate logic, allowing the DAQ board to provide $5 \mathrm{~V}$ DC output to only one of the solid state relays of the synchronizer buses at a given time. This guarantees the operation of the microgrid either islanding or in grid connected mode at one time. The mode can be changed by the islanding/synchronize switch from the front panel of the VI. The block diagram presented in the figure below explains the operation graphically. The other channels which are connected to front panel displays are for the controllable loads in the microgrid. These loads can be controlled by providing the $5 \mathrm{~V}$ DC output from the DAQ board. The control and monitoring for these is provided in the front panel of the VI.

Second part of the VI is to acquire the data from the main grid and the microgrid, helping in the connection of microgrid to the system. This part acquires the voltages and frequency on the main grid, it calculates the power drawn by the microgrid, power injected by the non-conventional sources and total power interchange. This part is also responsible for monitoring the voltage and frequency of the microgrid during the islanded mode of operation. Figures 5.4-1 and 5.4-2 present the Block Panel of LavView VI of Microgrid Interface and user Front Panel interface is presented in 5.4-4 and 5.4-5. 


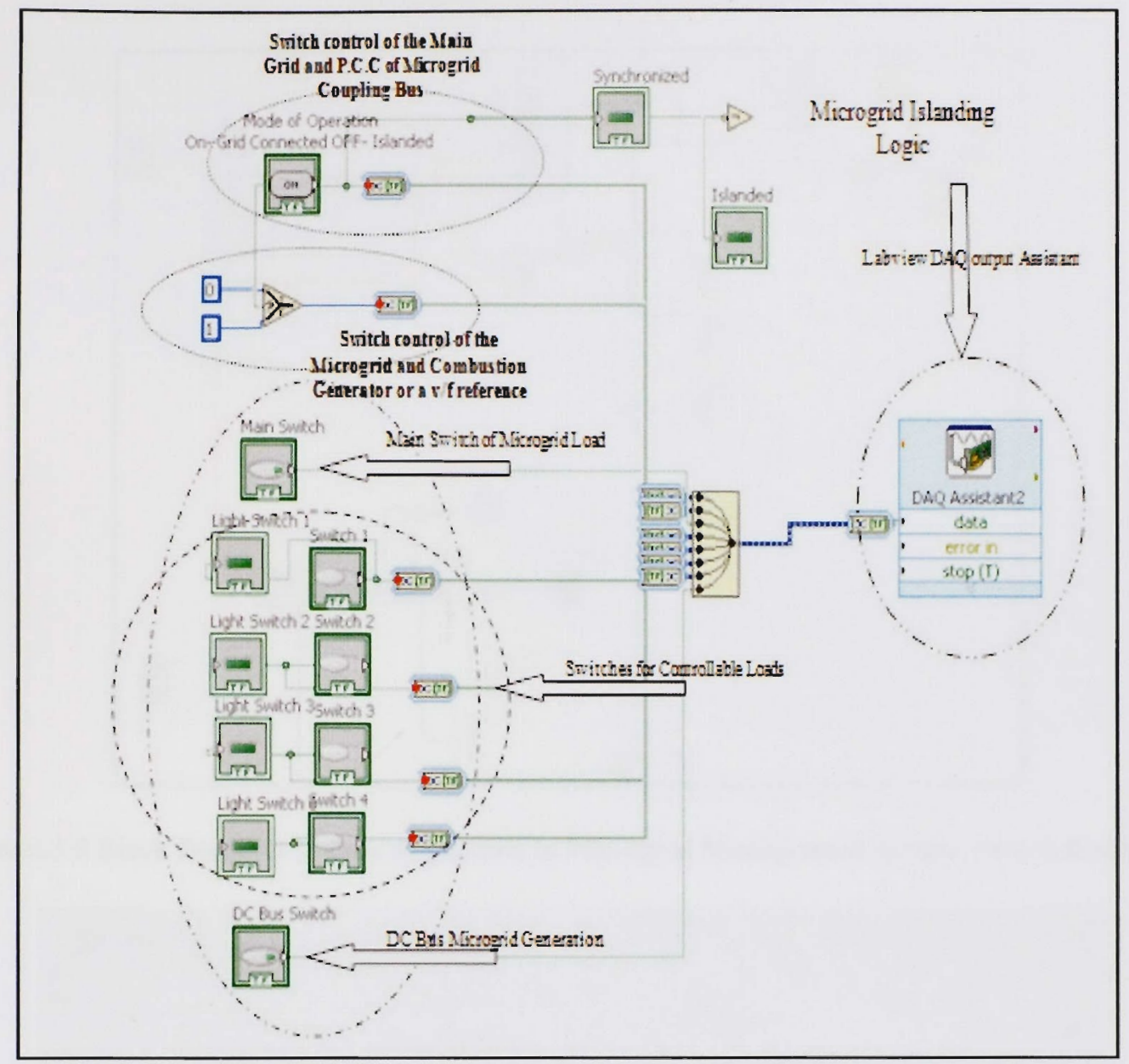

Figure 5.4-1 Labview VI program for Microgrid Control and Monitoring

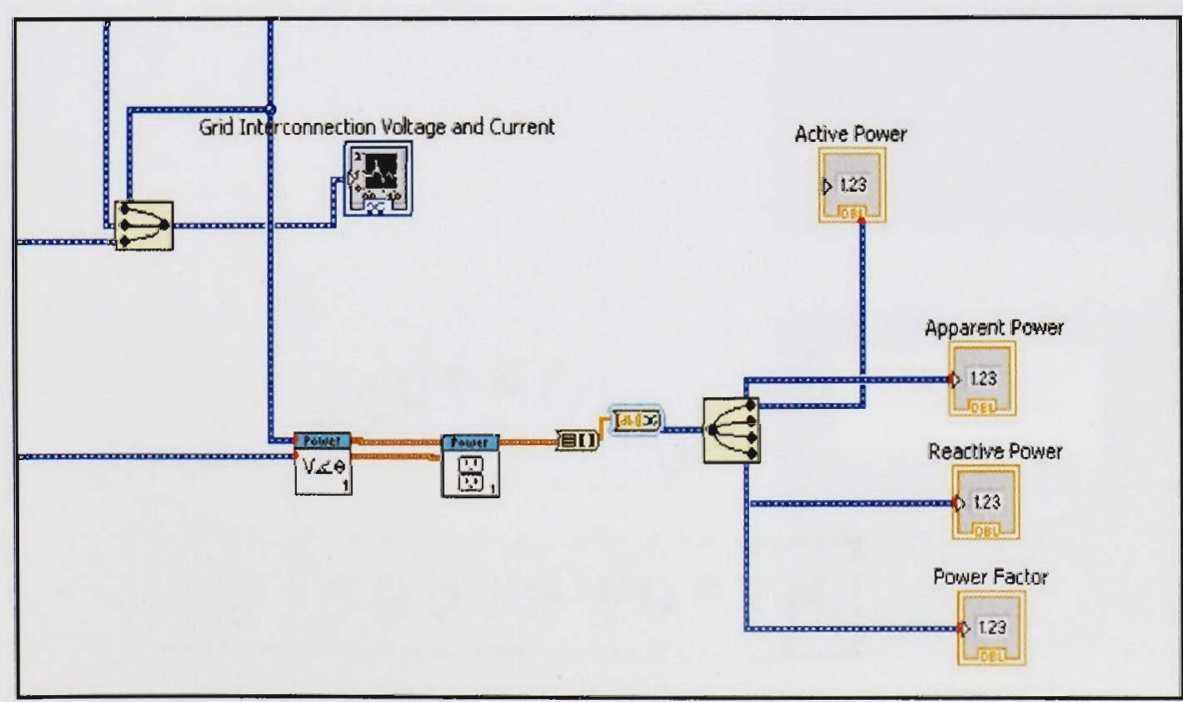

Figure 5.4-2 Block Diagram of Power calculation in Microgrid Management System VI in Labview 


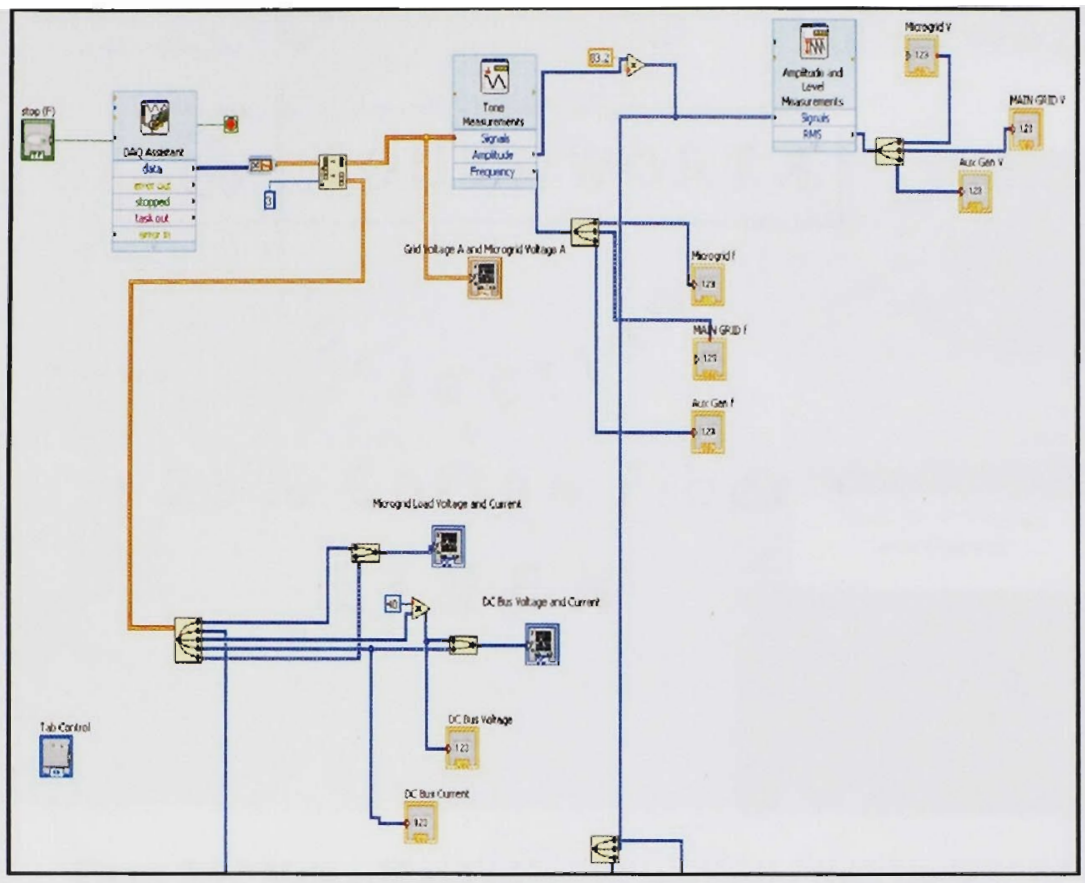

Figure 5.4-3 Block Diagram of data acquisition in Microgrid Management System VI in Labview

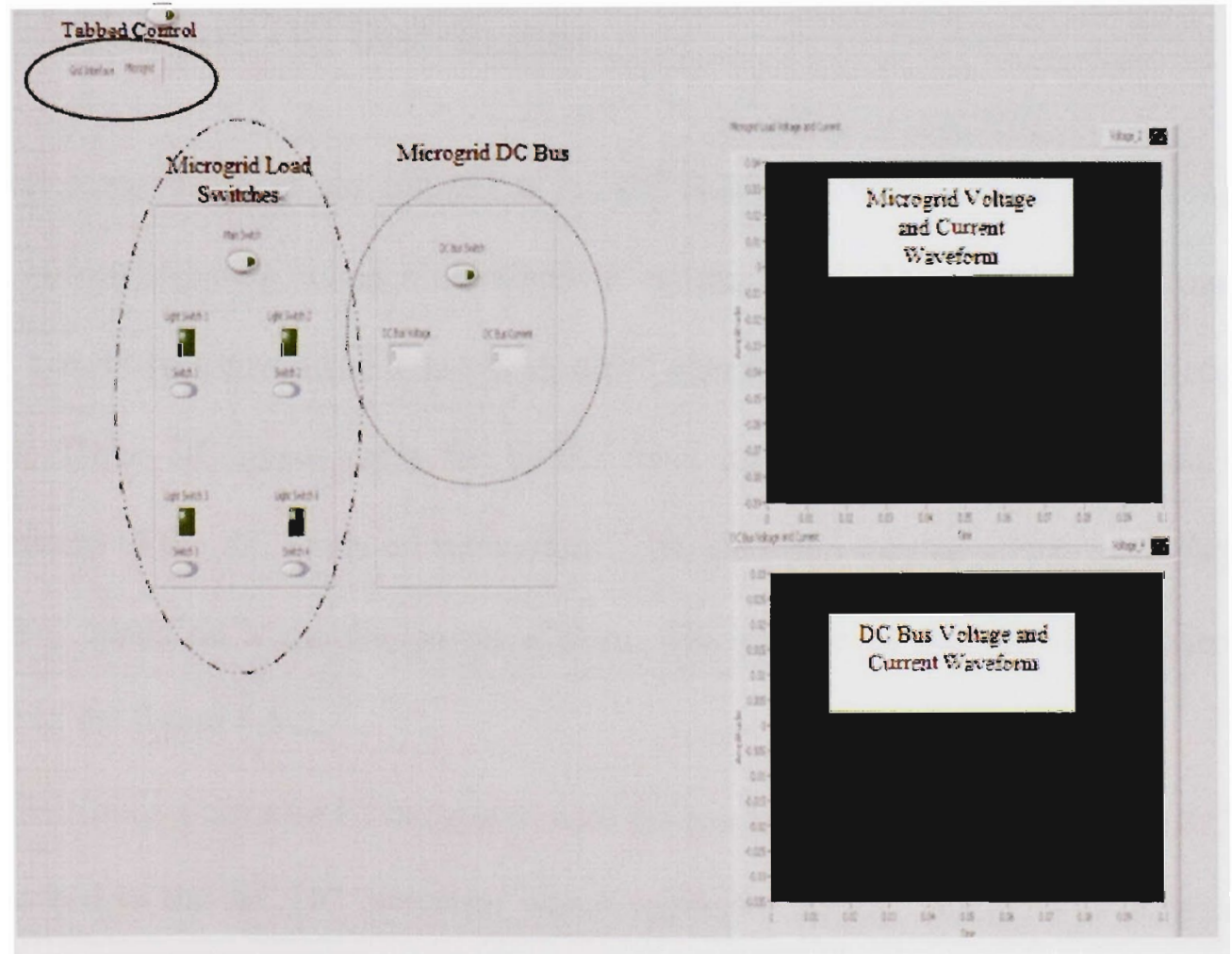

Figure 5.4-4 Front Panel of Microgrid Islanding and Load Management Control 


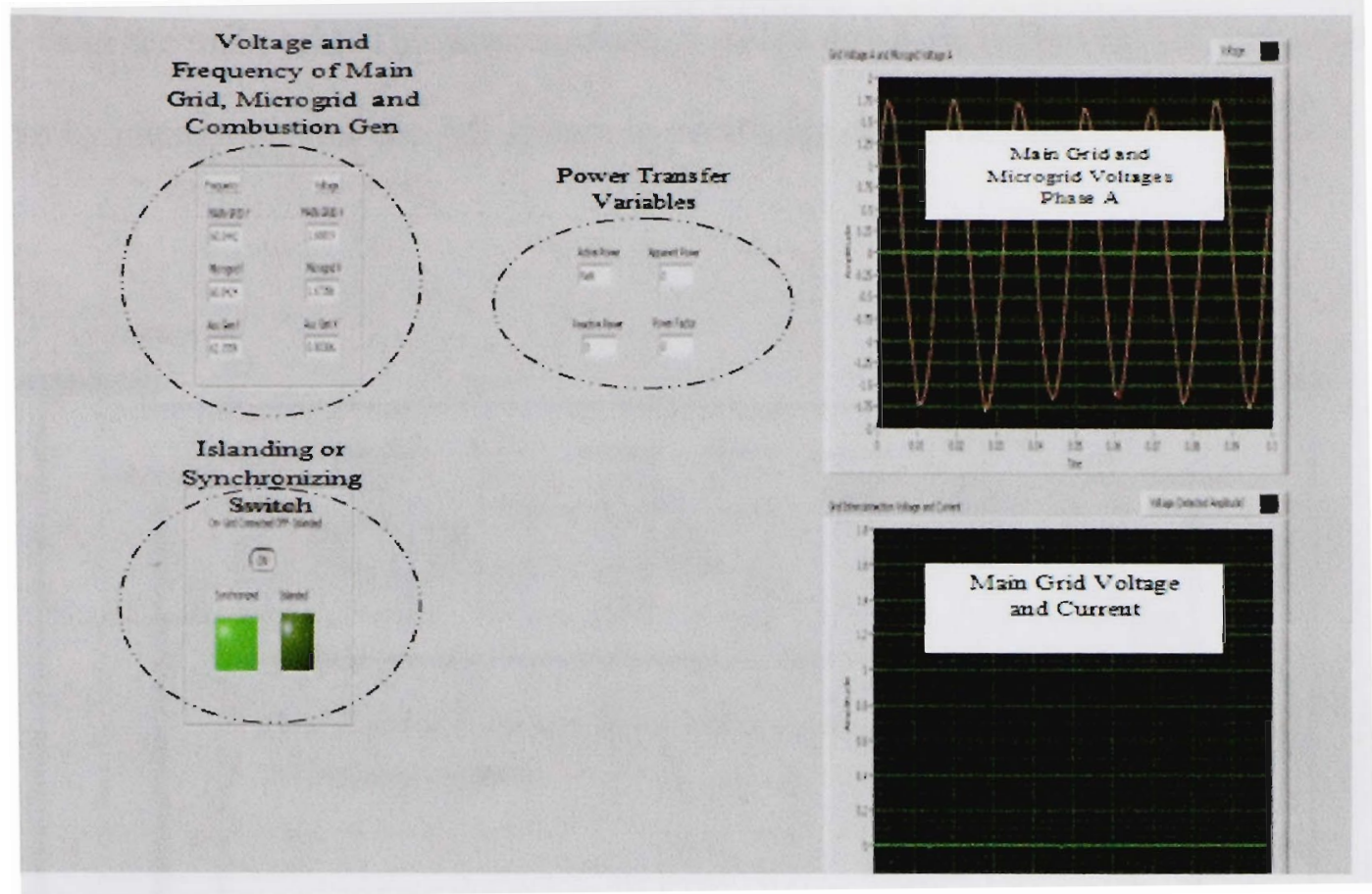

Figure 5.4-5 Main grid and microgrid monitoring front panel

\subsection{Bus and DC Distribution}

The alternate resources are coupled at the DC bus in the microgrid, as the microgrid contains various alternate resource emulators at various physical locations and also as the real time system will have distributed loads a DC distribution system is developed on the microgrid. Three DC buses carry the power from different alternate sources and are interconnected to the DC loads on the system. This also provides an added flexibility to emulate DC loads on a mobile power system. The picture of the DC distribution is presented in the figure 5.5-2.

The DC distribution can also be integrated with the test bed by interconnecting the P.C.C. at the test bed to the $\mathrm{AC} / \mathrm{DC}$ converter which regulates the $\mathrm{DC}$ voltage and the power 
input from the main grid. This also emulates a scaled down version of HVDC line on the system by interconnecting the AC system by rectifying on the DC side.

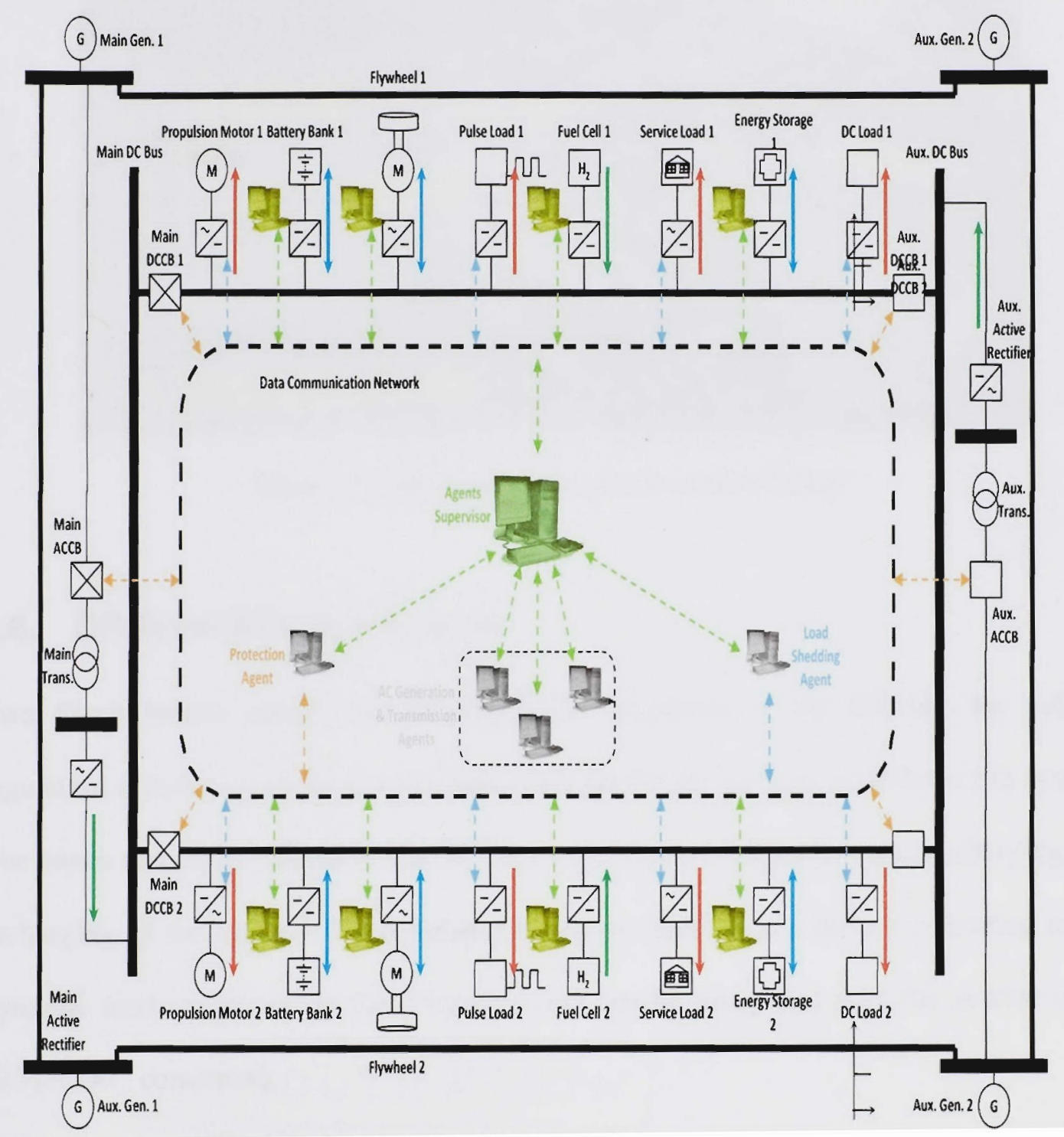

Figure 5.5-1 Block Diagram of DC Distribution system within the AC test bed 


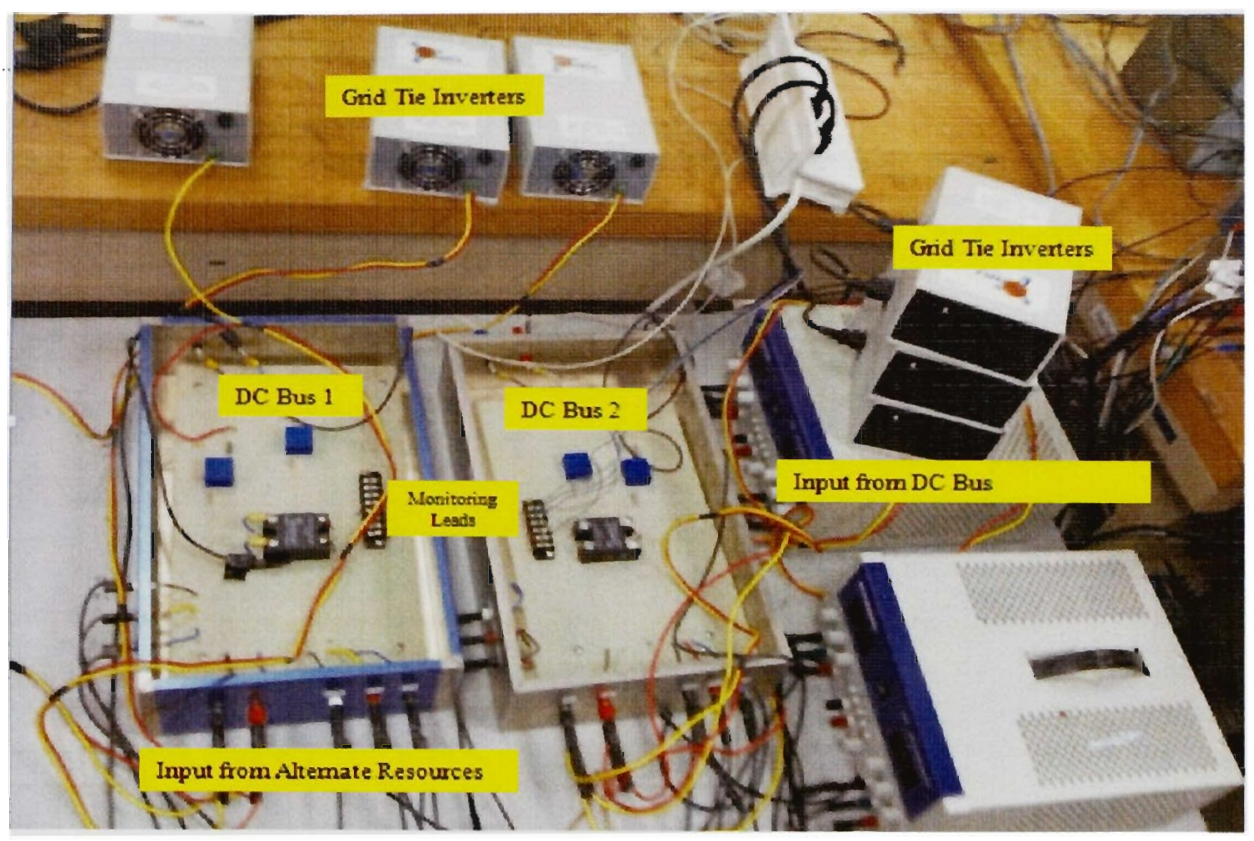

Figure 5.5-2 DC Distribution system on the Test Bed

\subsection{DC level Storage System}

Two 5kwh battery banks are develop to be connected to the DC bus for voltage regulation a during peak load and energy storage during no load periods on the system. The banks can be connected to the DC bus with trickle chargers for online charging and recharging of the system. A mechanical flywheel model is available for catering to the dynamic load variations on the microgrid and can be integrated with the system using $\mathrm{AC} / \mathrm{DC} / \mathrm{AC}$ converters. 


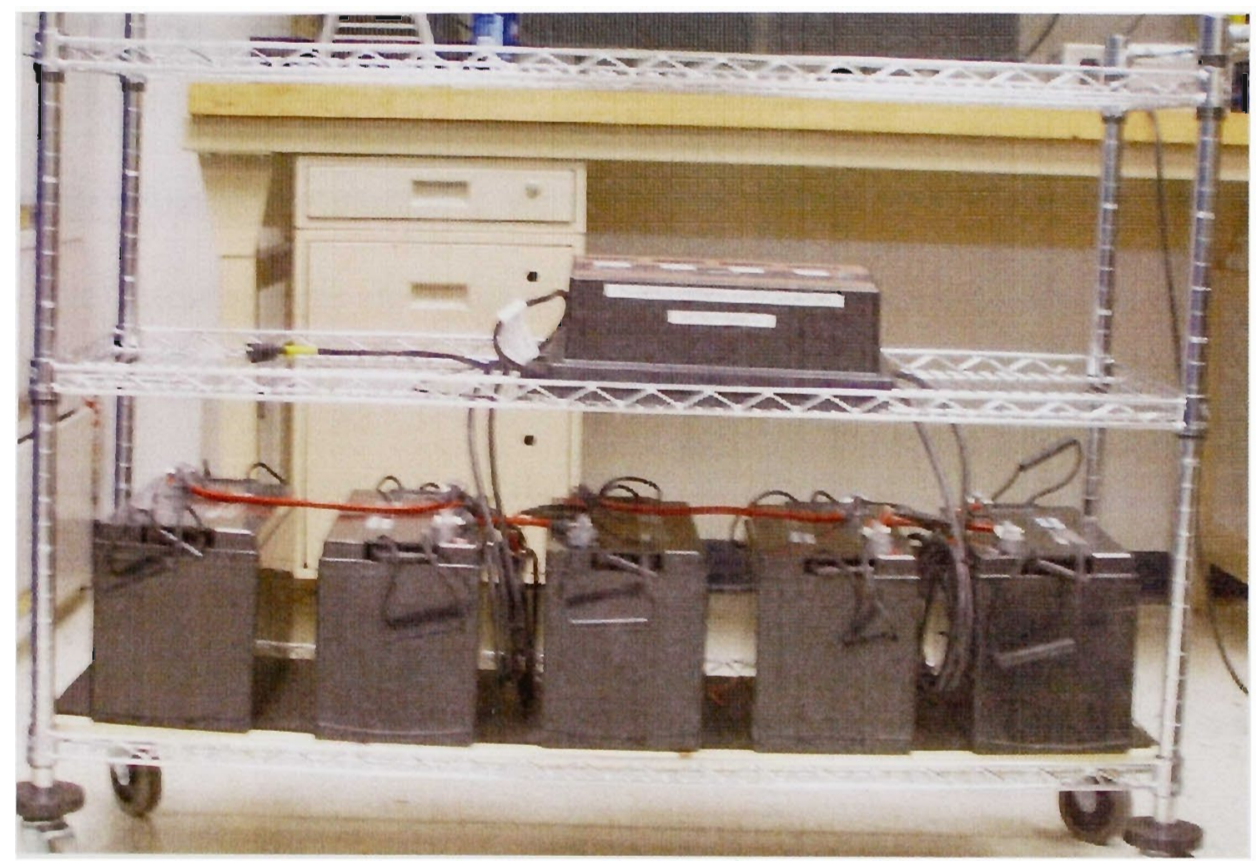

Figure 5.6-1 Battery Bank array

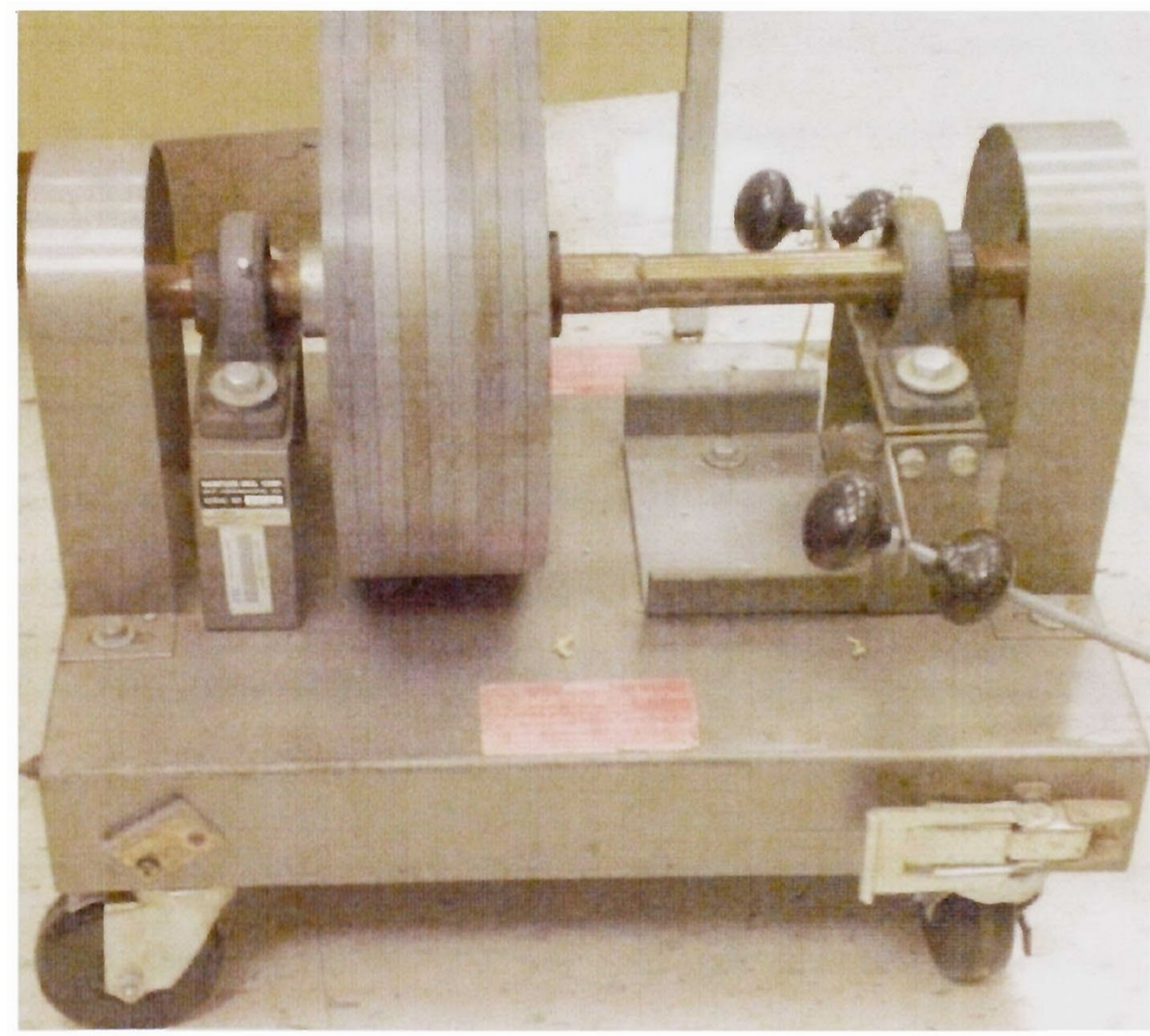

Figure 5.6-2 Flywheel Model 


\section{CONTROL AND COMMUNICATION INFRASTRUCTURE}

This chapter presents the modeling and design of control and communication layers. It presents the infrastructure designed for control framework and the devices. It also presents experiments done to implement communication framework. Lastly details on administration and management of the Multi Agent System framework installed and implemented as a part of power system test bed and microgrid platform.

\subsection{Control and Data Acquisition Devices used on the Test Bed}

The SCADA system and the control system for the power system platform are closely related in this work. Matlab and Simulink are used for developing the control logic presented in the earlier sections. Real time workshop in Simulink is used to developing the model and then integrating with dSPACE 1104 control board. Real time workshop has options of converting the Simulink model into $\mathrm{C}$ or $\mathrm{C}++$ code, this code is later burned into the Digital Signal Processor (DSP) in the control board. The dSPACE control board is managed by the dSPACE control desk software which is used to develop the GUI (Graphic User Interface). The dSPACE 1104 control board has 8 manual A/D (analog to digital) and D/A (digital to analog) channels, it also has a 16 channel PWM generator, RS485 and RS422 UART's (Universal Asynchronous Receiver and Transmitter).

Labview software and DAQ boards NI6025E, NI6071E and NIUSB6259M are used for control in the synchronizer module and microgrid management system. Labview software has two panels, the block diagram and the front panel. The block diagram acts 
as the code for the system and the front panel is similar to a GUI. The difference between dSPACE and Labview is response time, Labview is comparatively slower than dSPACE but as it is also cheaper, it is suitable for applications not requiring fast response, on the other hand dSPACE is expensive but it is a very good way for application to systems requiring faster response and higher accuracy.

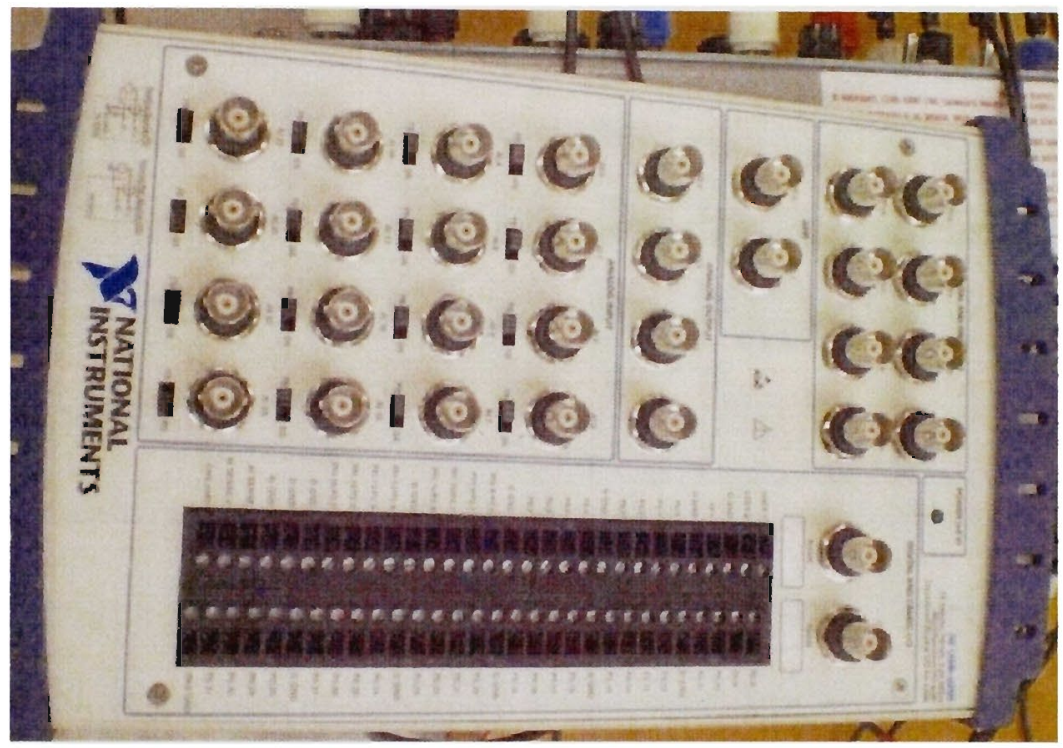

Figure 6.1-1 NI 6259M BNC Test Bed AC system Control Board

\subsection{Communication Components and Technologies Implemented on the Test Bed}

Communication is an important part of the test bed project. Proper control depends on reliable communication. Few experiments were conducted to verify the effectiveness of the communication layers which could be implemented for the present components of the power system. Xbee module employing the Zigbee IEEE 802.11 protocol was one among them. A virtual instrument was built in Labview to feed the data acquired from the generator bus and send it to the Xbee module, although the interface was functioning properly and the data was being sent from the Xbee sender module, the data was not 
received at the receiver module and was being lost. Zigbee protocol is for serial and unreliable communication and hence it was felt that it was not effective to utilize Xbee module for communication in control system. It can still be used for monitoring purposes as data loss will not account for any stability concerns in these kinds of applications.

The present architecture of the power system test bed employs Ethernet based communication, although it is wired communication a wireless router can be employed in case of wireless applications. This network works based on the Labview data server and web server. The web server is used for publishing the Virtual Instruments in Labview on the internet which can be later monitored or controlled from anywhere on the globe. The variables on the virtual instruments can also be shared using shared variables in Labview. The next important concern is to develop a network of the generating stations connecting the motor drives in a ModBus protocol based network. The software interface for this network has been developed in Labview, with virtual instruments created for sending signal and receiving signal from the drives.

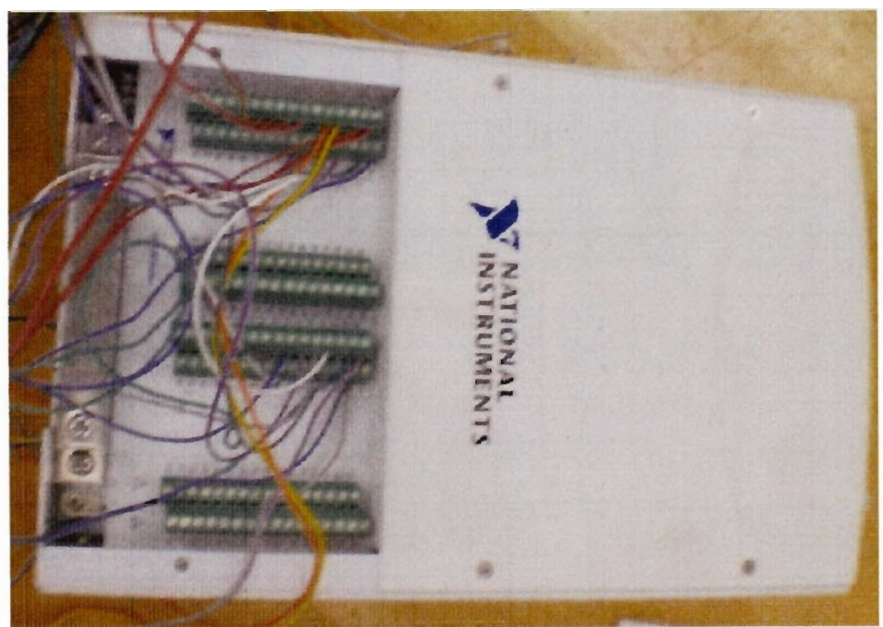

Figure 6.2-1 NI 6259USB DAQ system used for Microgrid Interface VI in chapter 5.3 


\subsection{Lenze Motor Drive Communication}

The electric drives used for controlling the alternators are equipped with ESVZAR0 RS232/485 based ModBus and LECOM communication module. The control of these drives will enable in remote monitoring and control of the frequency of the alternators. The hardware wiring followed for both the protocols is same and the drives have been hardwired according to the protocol based manner. The selection of the protocol is based on the TechLink or any other software interface. The 5 pole connector of the ESVZAR0 module is used with a 2 wire connection and RS 485 protocol. An RS 232 to RS 485 converter is used for wiring the drive to the computer. The input terminal of the drive is cross connected at transfer and receiver terminals with a $120 \mathrm{Ohm}$ resistor for reducing the noise. Twisted pair cable is used for connecting the drive and the RS 232-485 converter as it causes a very low noise. Figure 6.3-1 presents the terminal connection on RS 485 terminal and (It is to be noted that TX-transmistter on drive becomes RX-receiver on computer and vice versa) drive terminals.

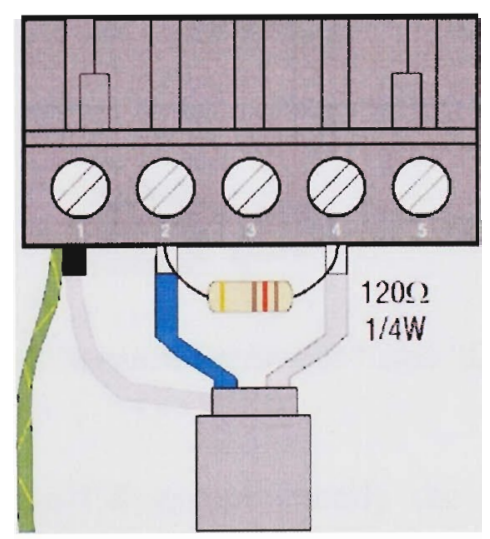

Figure 6.3-1 Drive Terminal Hardware Connection 
The drives of the network are connected in serial order and in a ring bus structure. The advantage of ring structure for communication with drives is increase of reliability with a closes loop. The commands for the drive are broadcasted on this network and the respective drive is identified through the unique network address according to the program developed. The protocols followed for sending and receiving data are based upon the protocol followed among ModBus and LECOM. A block diagram is presented which explain the network connection of the drives.

ModBus is a asynchronous serial protocol designed for industrial communication applications. The ModBus RTU architecture is based upon a PLC to device communication structure and, as such is Master-Slave in orientation. The SMV drive in this case acts as the slave in this network, responding to commands and requests from the master computer. Figure 6.3-2 presents the flow of commands.

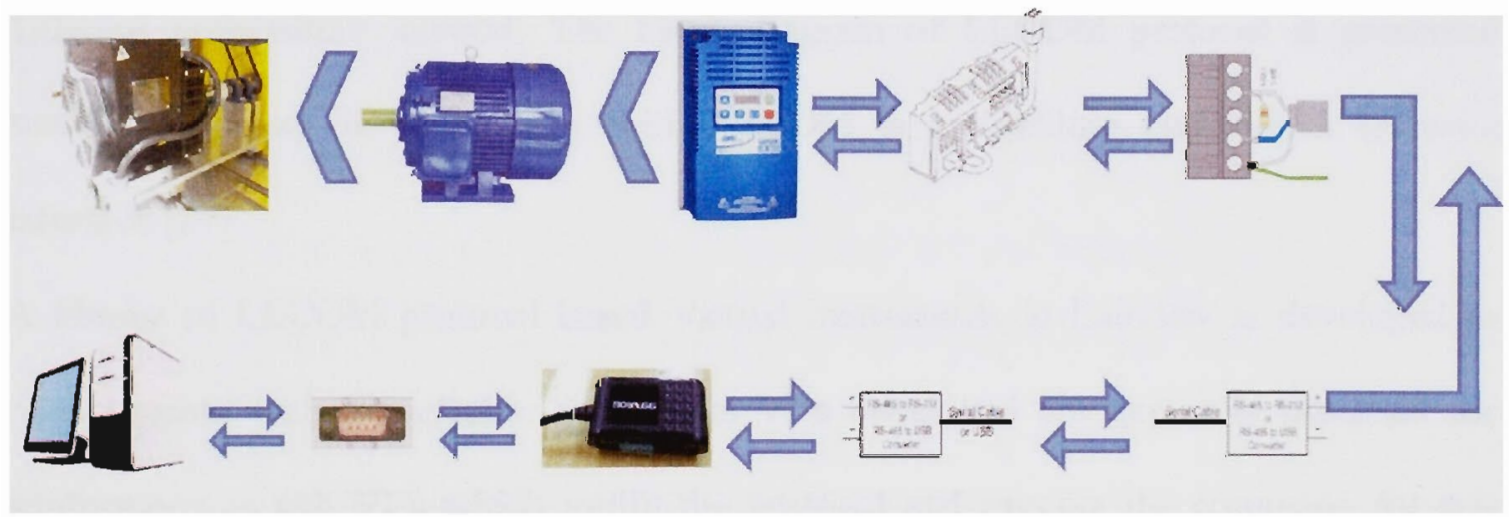

Figure 6.3-2 Network Control of Lenze Motor Drive

While the ModBus RTU protocol does not specify the physical layer, the ESVZAR0 module uses the RS 485 protocol.

Typical Commands which can be given between the master and slave are:

- Run command from Remote Computer on ModBus Network. 
- Frequency Reference

- Reporting of Fault of Drive to the Master

- Request for regeneration Voltage from the master

The drive has 20 parameters for communication for operations varying from network control, baud rate adjustment, network address setting etc. The manual for ESVZARO module provides further details [26]. The ModBus protocol is used through Techlink software package. The application allows programming the drive address and communicating for receiving and sending commands. However, a drawback with this application is that it there is no interface to send and receive commands through any of the control devices mentioned earlier and hence the following LECOM protocol is reviewed.

LECOM protocol is better than ModBus due to its speed, higher baud rate support and different architecture support. The block diagram of LECOM protocol is presented below. However, the complexity with LECOM is in building customized software interface [27].

A library of LECOM protocol based Virtual Instruments in Labview is developed to communicate with the electric drive. The VI's developed are generally designed for applications as sub VI's which verify the protocol and execute the command for any application. As the commands are generally to acquire data and send data, the VI's are branched as 'askVI' and 'sendVI'. The concept here is to create a separate interface library which can be used with any experiment created in Labview. 


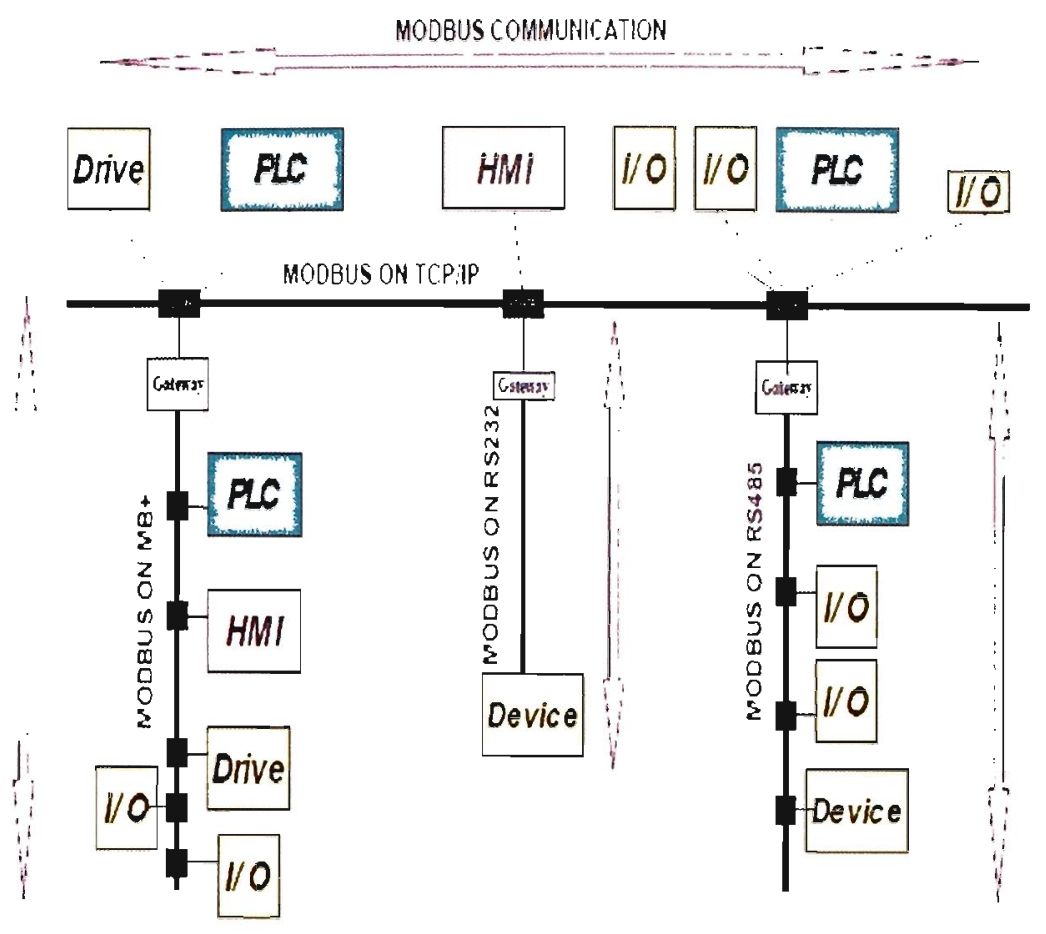

Figure 6.3-3 LECOM Protocol

The list of functions of VI's developed as a part of the library for implementing the LECOM protocol on the test bed is as follows:

- Acquire Speed, Frequency, Voltage and Current at the Drive.

- Perform Network Start, Stop, RUN and pause operations from the RTU.

- Provide Speed, Frequency, Voltage and Current Reference for the Drive.

- Acquire Active Power, Reactive Power and Regenerative Power at drive terminals.

- Reset Fault, Provide Set point for frequency, voltage and current tripping and override internal relaying.

- Implement PID control and PID set point. 


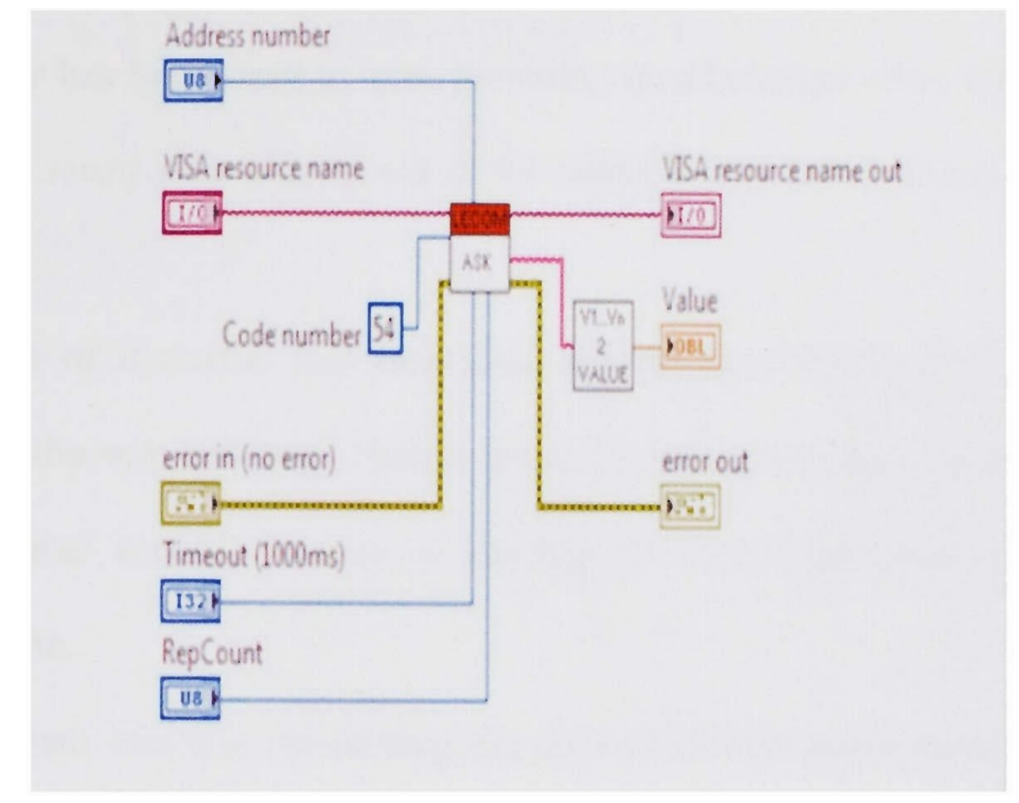

Figure 6.3-4 Block Diagram for Data Acquiring VI for LECOM protocol

\subsection{SCADA system in Labview}

The previous sections presented the communication layer from components to the control computer by using DAQ system and communication between the RTU and motor drive for generator control. Another critical layer of communication is between the various components on the test bed, inter-process and inter-component communication. Labview software package used for control and DAQ and mentioned in the previous sections provides platform called Shared Variable for sharing the information between two applications. It is clusters the variables to a same value on the network and the data transfer is facilitated using a TCP/IP network. A TCP/IP network is implemented on the test bed for this application. The values of the variables are buffered on the sending 
system. The time delay is affected by the DAQ board used on the transmitter side, the size of the variable buffer and the TCP/IP network in the background. To reduce the time delay the buffer has been sized to zero providing an advantage of no time delay due to buffer but very rarely data can be lost if the sending application rate is faster than the receiving rate.

There are a lot of dispersed and individual applications developed for control and monitoring on the test bed (e.g. Synchronizer modules, microgrid interface, alternate emulators). Shared variable is used to interlink all these applications for a common database platform.

A SCADA system interface monitoring the data of all the applications on the Master control computer is developed on the test bed. The interface acquires the data of various distributed applications on the test bed and can monitor and control the applications from remote system. This provides a great benefit of distributed control from a clustered common unit. The data can be stored in an excel sheet or a MySql database format for further evaluations and stability studies of the test bed. It also helps in emulating faults and contingencies on the system to evaluate response, by disconnecting transmission lines and buses, closing relays and removing generators from a remote location. The user interface panel of the SCADA system is presented in figure 6.4-1. 


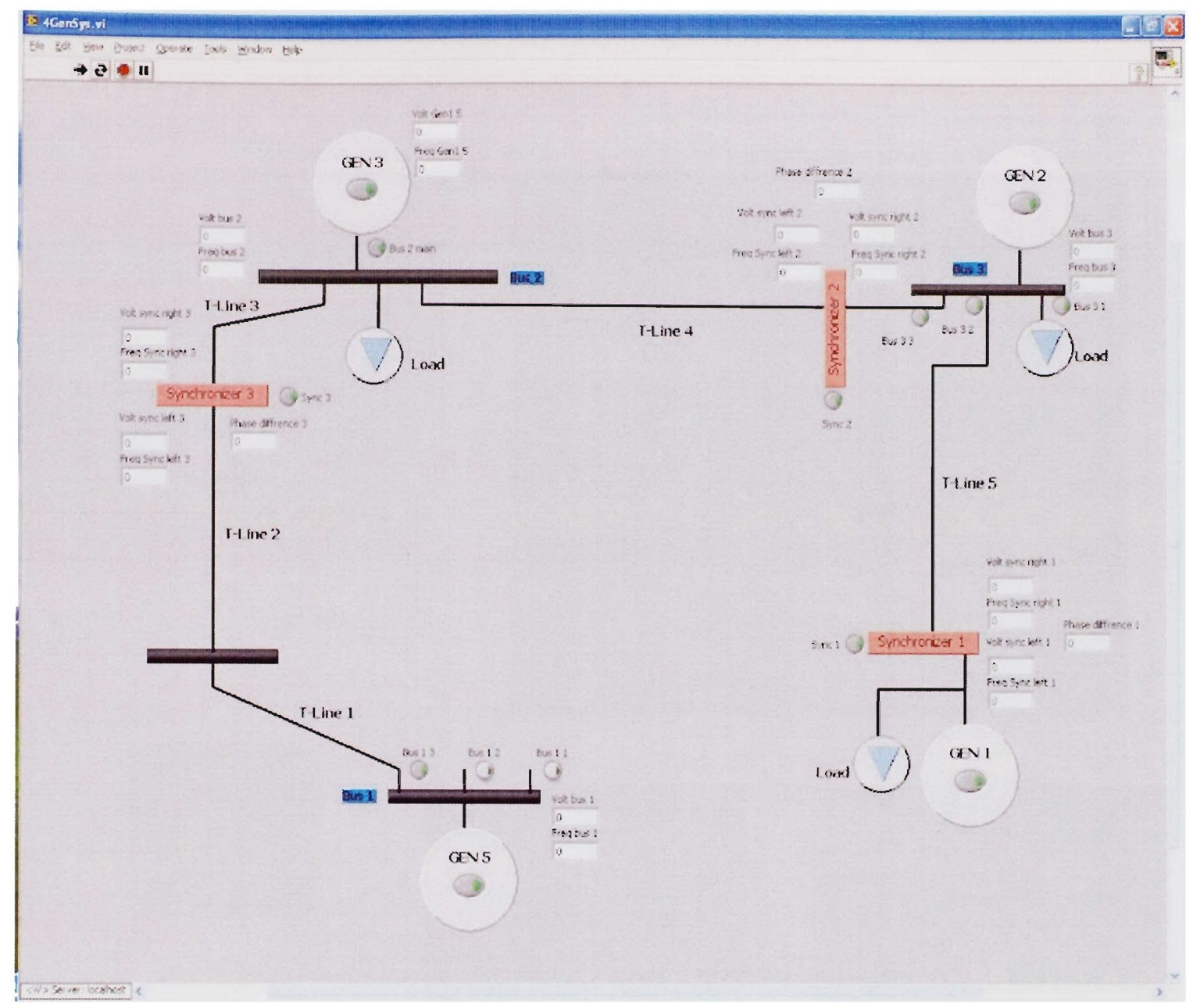

Figure 6.4-1 User Interface of SCADA system of the Test Bed

Labview package provides a data socket server and web server which can be configured for publishing data to the internet. Data Socket server is used for sending and receiving data packets on the internet using the TCP/IP platform. Figure 6.4-2 presents the LabView VI published as a web page, on the Internet Explorer web browser. 


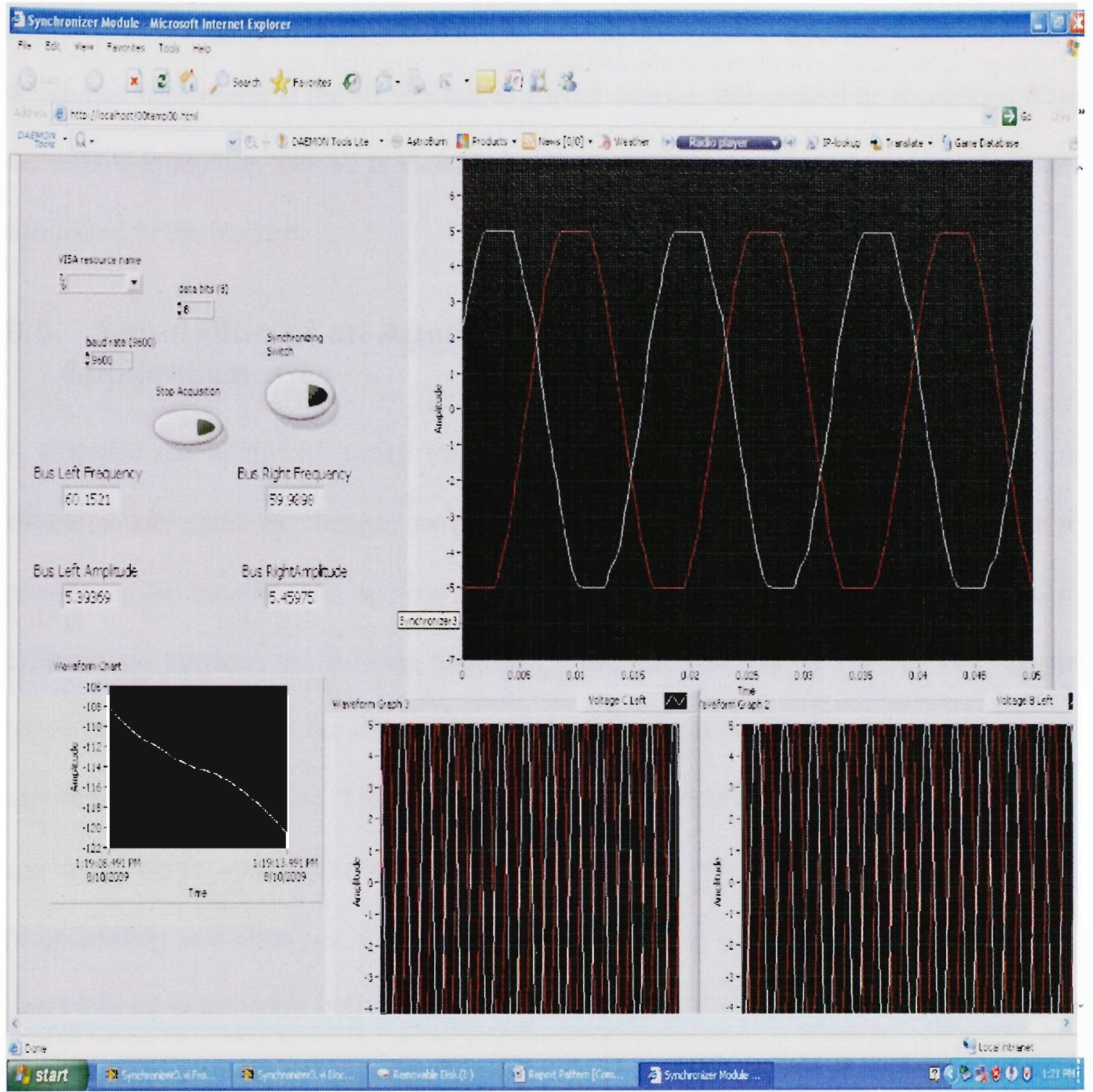

Figure 6.4-2 Synchronizer Module VI published on the Internet (Internet Explorer is used for viewing the Web Page VI).

The LabView web server can be configured and programmed to publish this data on the internet. This is a very critical application as it allows the consumers with alternate energy resources to monitor the energy data from remote computers and it also allows to independent power producers to control their energy input and output from the microgrid from remote computers on the globe. This feature of Labview is used for building two 
applications for monitoring and control. The programming for the web server on the VI developed determines if the VI is allowed partial control, full control or no control from the remote computer. The VI is viewed exactly as a web page from any internet browser connected to the internet.

\subsection{Simulation of an Agent Framework for Test Bed Application}

A software (or hardware) entity that is situated in some environment and is able to autonomously react to changes in that environment is defined as an agent. Prior to presenting the benefits and application areas of multi agent systems, it's important to differentiate between the existing technology and the multi agent system. The present control systems acquire the data and implement the logic which has been programmed according to the algorithm. The conceptual difference between the present control system and multi agent system is that control system takes just one decision based on the programming and does not take any independent decision. On the other hand a multi agent system is provided with details for actions but not on when to be taken. The multi agent system assesses the situation from the data acquired and based on the data it will take and independent decision. Although it has communication from the other agents in the system, it does not need to be aware of other zones in the system for which the particular agent is not responsible. The control here is decentralized as there is no master controller. It is equivalent to a human controlling and decision making process. The figure below explains the concept of application of multi agents systems in a power system. 


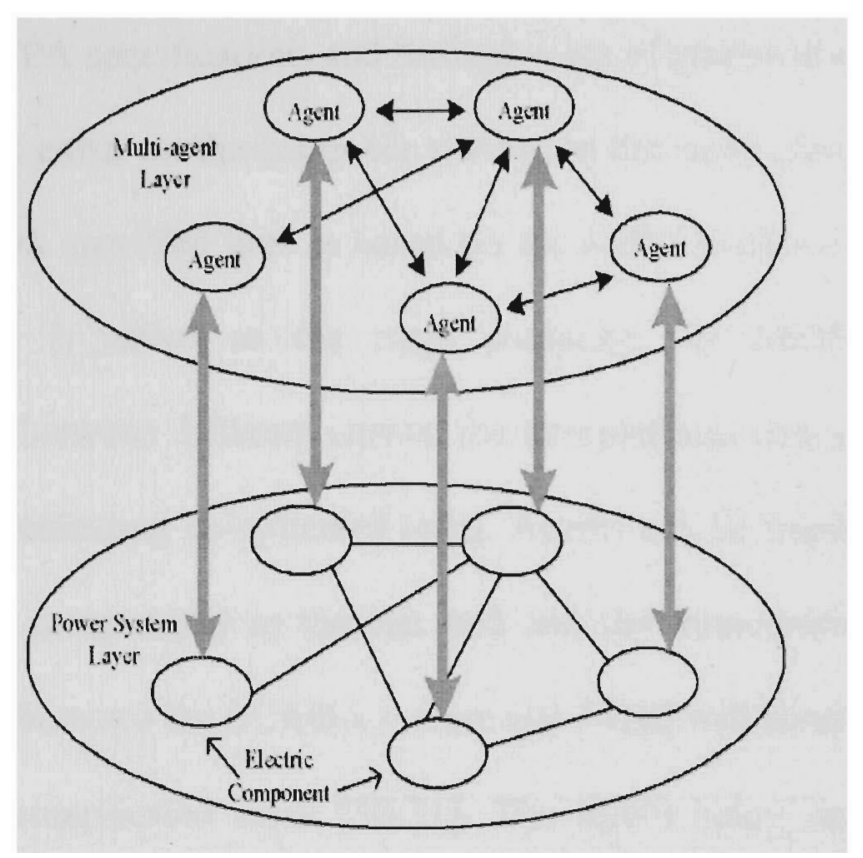

Figure 6.5-1 Multi Agent System application in power systems

There are a lot of benefits obtained by addressing the control based on multi agent System related to time of response, reduced response times and distributed control. The goal of this thesis was to develop a platform to implement Multi Agent System (M.A.S.) based control. Although there are many definitions of agent systems, some defining dSPACE and Simulink based systems also as agents. The idea of this thesis was to stick to the definition of the agent presented in the work of Stephen McArthur et al [28-29].

There are many platforms which can be used to implement agent systems like JADE (Java Agent Development Framework), etc. It was decided upon to use JADE as it conforms to FIPA (Foundation for Intelligent Physical Agents) specifications. A plug in called eJADE allows Eclipse IDE (Integrated Development Environment) to utilize all features of JADE in a GUI based environment.

JADE is a software framework fully implemented in Java language. It simplifies the implementation of multi-agent systems through a middle ware that 
complies with the FIPA specifications and through a set of graphical tools. JADE allows installation of agents either on the computer systems in the same network or on different network. The network specified here is based on the JADE platform; the system which hosts this platform is called as the main container. As JADE also allows for intercommunication between different agents, the interplatform communication between agents helps in implementing complicated tasks. Agents can be implemented at various levels and diffcrent components in the test bed and the intercommunication between agents and interface between the SCADA system and JADE will benefit in implementing control system for complicated areas [30-31]. The figure below represents the block diagram of agents and interconnection with power system components.

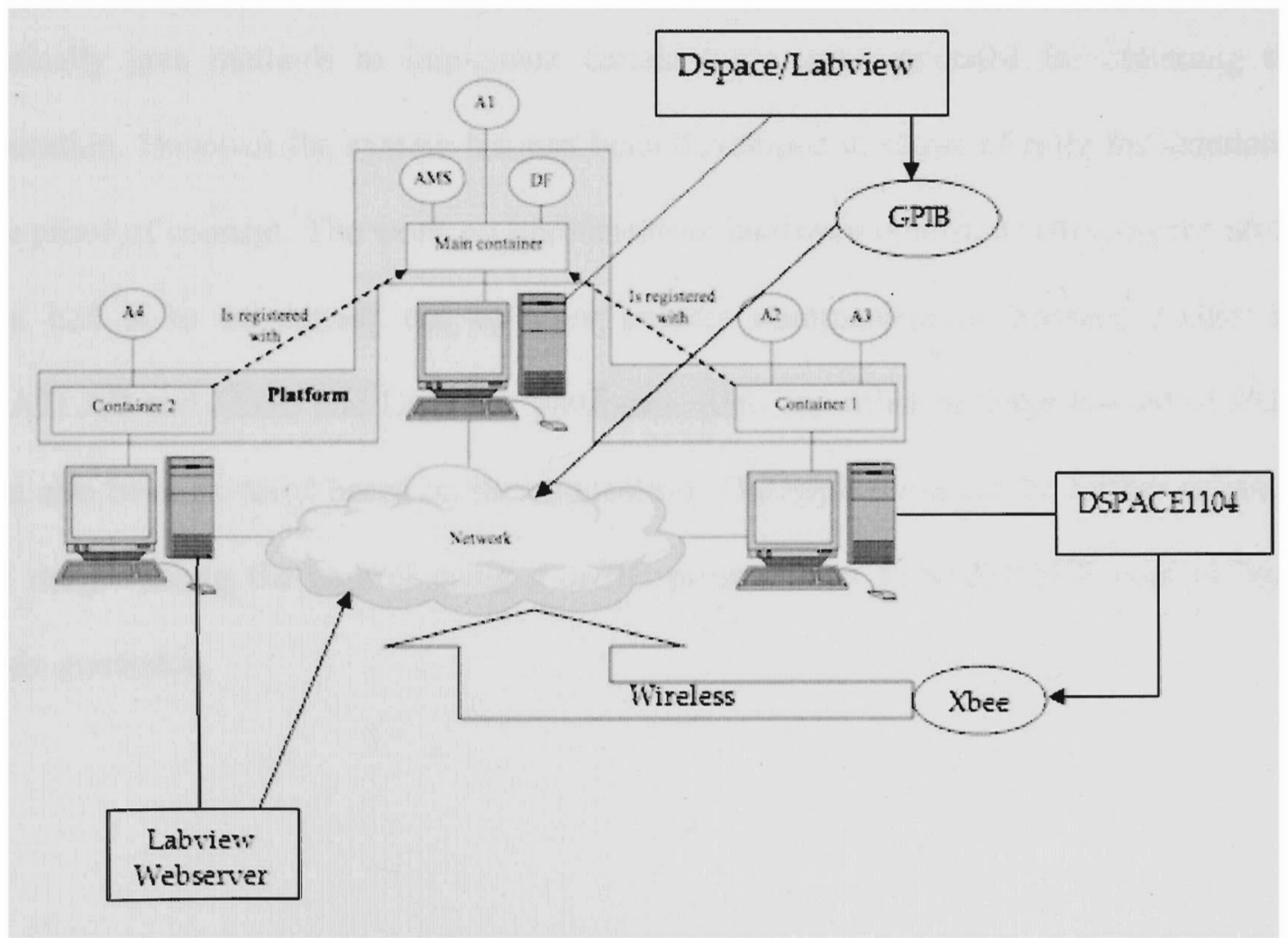

Figure 6.5-2 Multi Agent System application in power systems 


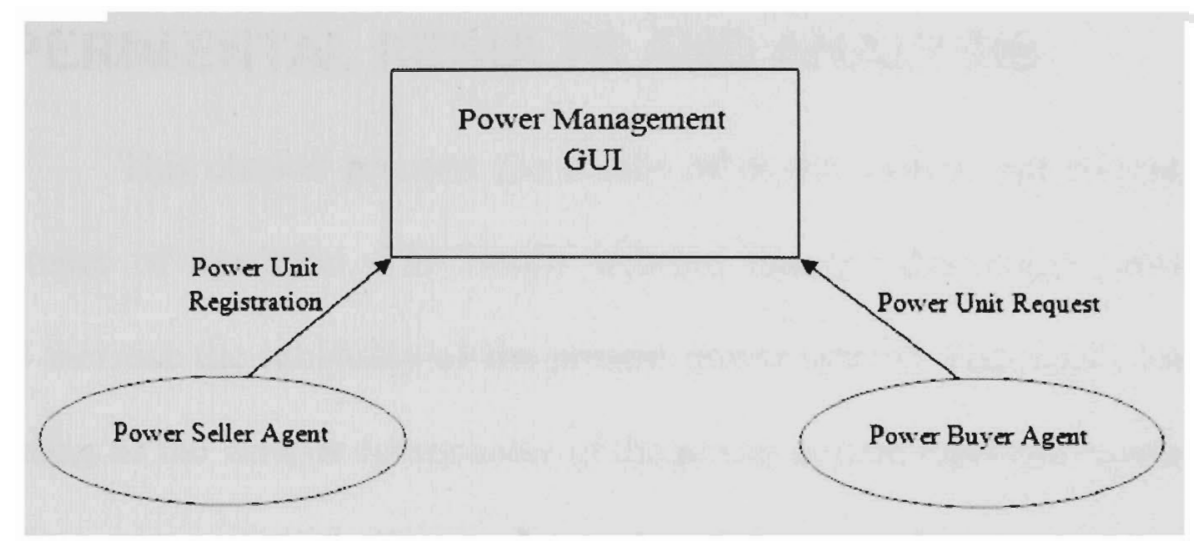

Figure 6.5-3 Energy Management System

An agent based energy management system was developed to present the multi agent system logic. A power buying agent, which is registered with the management system representing the main grid, power selling agent representing a microgrid and registered with the management system were developed. The behaviors in the JADE framework, basically java methods to implement certain logic were extended for obtaining this operation. However the system has just been developed in terms of code for simulating the proof of concept. The work on implementing hardware control of components on the test bed is to be carried out by inter process communication between JADE and MATLAB and JADE and LabView platforms. Also any other package instead of JADE can also be considered based on the application. The application can be further enhanced by programming the control using a dsPIC programmed from dSPACE control board code generator. 


\section{EXPERIMENTAL RESULTS AND ANALYSIS}

This chapter presents the results of power system and microgrid during various stages of operation. The results indicate that the distributed generation and microgrid increase the reliability of the present power system. The results also indicate that modeling of the various components of the power system represent similar behavior of a modern power system. Hence, the study of dynamics by integrating distributed resources and techniques will provide true values as in case of a modern power system.

The following experiments are case studies conducted on the test bed. These experiments were conducted in order to determine the stability of the power system developed as a part of this thesis, its response to various events. Experiments to verify the benefit of distributed generation and microgrid, and connection of microgrid to power system are also conducted.

\subsection{Experiment 1: Energy Sharing between Test Bed and}

\section{Microgrid}

\subsubsection{Experimental Setup and Results}

This experiment was conducted to study the energy sharing between the main grid and energy from the alternate sources. A partial setup of the test bed with resistive load was connected to the microgrid model through the grid tie inverters and a DC power supply was used for emulating alternate resources. The setup is presented in the figure below. 


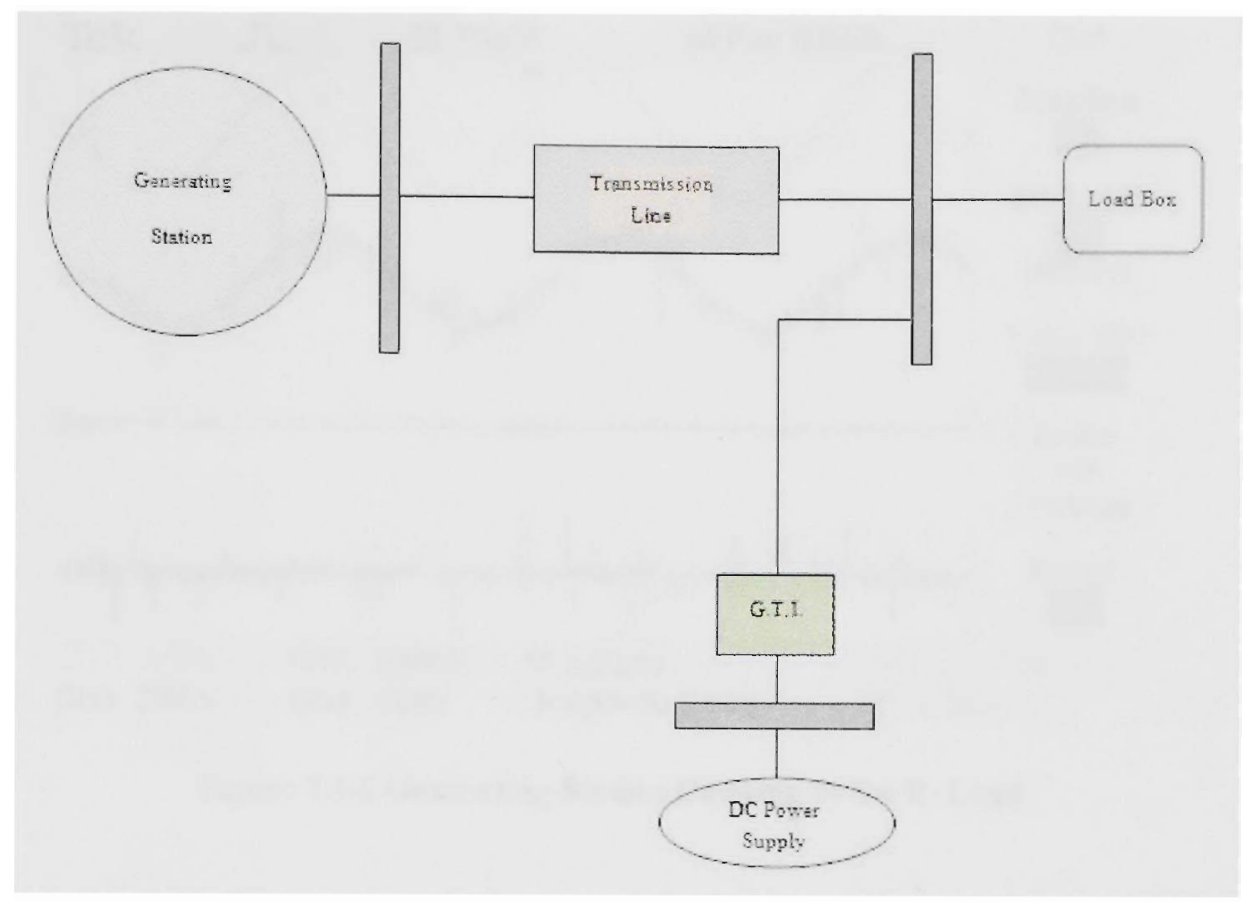

Figure 7.1-1 Power Sharing with Resistive Load

Initially only the Generating Station is catering to the load and GTI is disconnected from the system. The voltage and current waveforms of the system are presented in the figure below. The DC Bus voltage, current. Load bus voltage and current waveforms are presented in the following figures 7.1-2, 7.1.3 and 7.1-4. The waveforms present the instantaneous values obtained from the $\mathrm{CT}$ and PTs and the conversion ratios for these measurement devices can be obtained from the appendix $b$ from the bus bar section. Also the real time RMS values obtained are presented in the analysis section. 


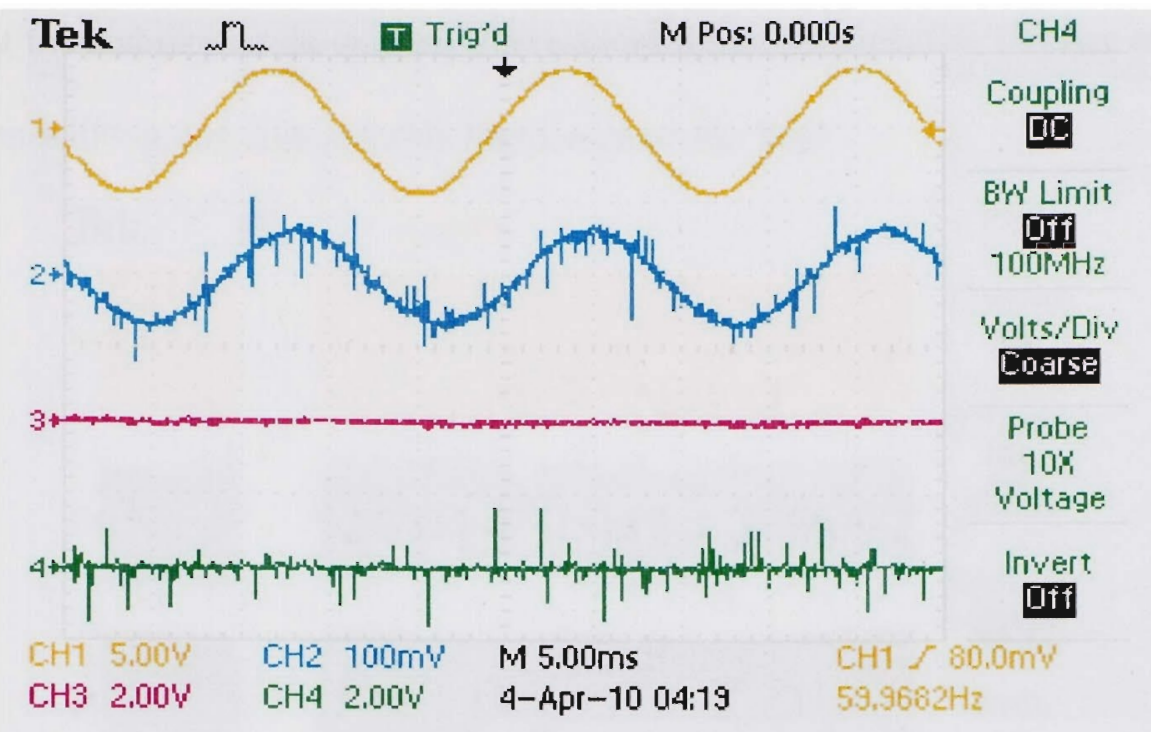

Figure 7.1-2 Generating Station Catering to the R-Load
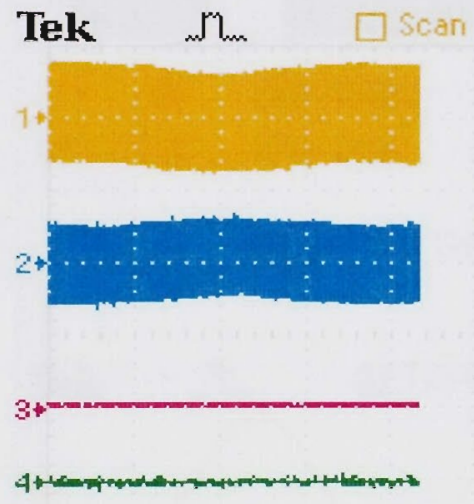
$\mathrm{CH} 72,00 \mathrm{~W}$
CH3 1.000

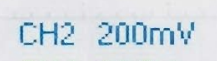
[CHA 10.0W
M 5.00s
4-мрг-10 04:39

$\mathrm{CH3}$

Coupling

BW Limit

DIff

$100 \mathrm{MHz}$

Volts/Div

Coarse

Probe

$1 \mathrm{x}$

Voltage

Invert

Dff

Figure 7.1-3 Steady State Response of Gen connected to R-Load

The GTI is then turned on with the load connected to the system. Two figures present the scenario is the system after connecting the GTI. Firstly the steady state waveform presents the frequency and the voltage waveforms over a period of time 
displaying the stability of the system. The second figure presents the Voltage and Current waveforms without any considerable harmonics on the load.

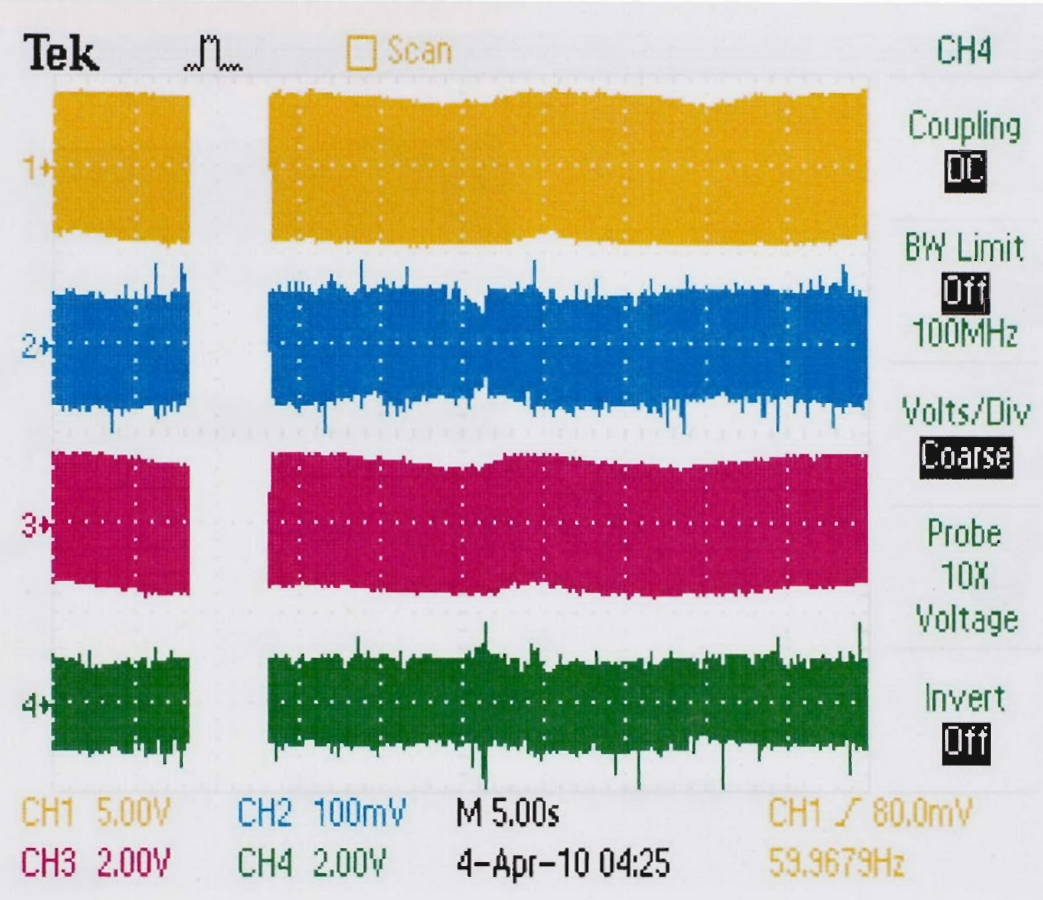

Figure 7.1-4 Gen and GTI sharing R-Load

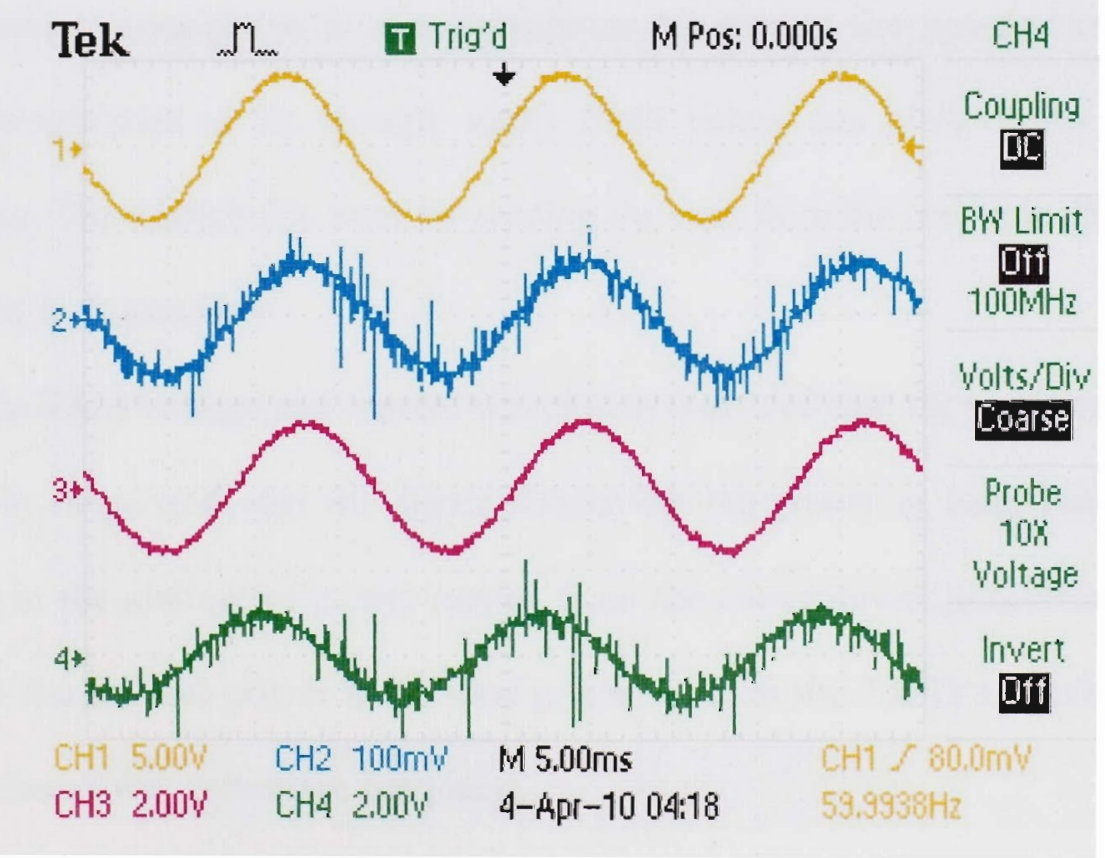

Figure 7.1-5 GTI and Gen Sharing R-Load 


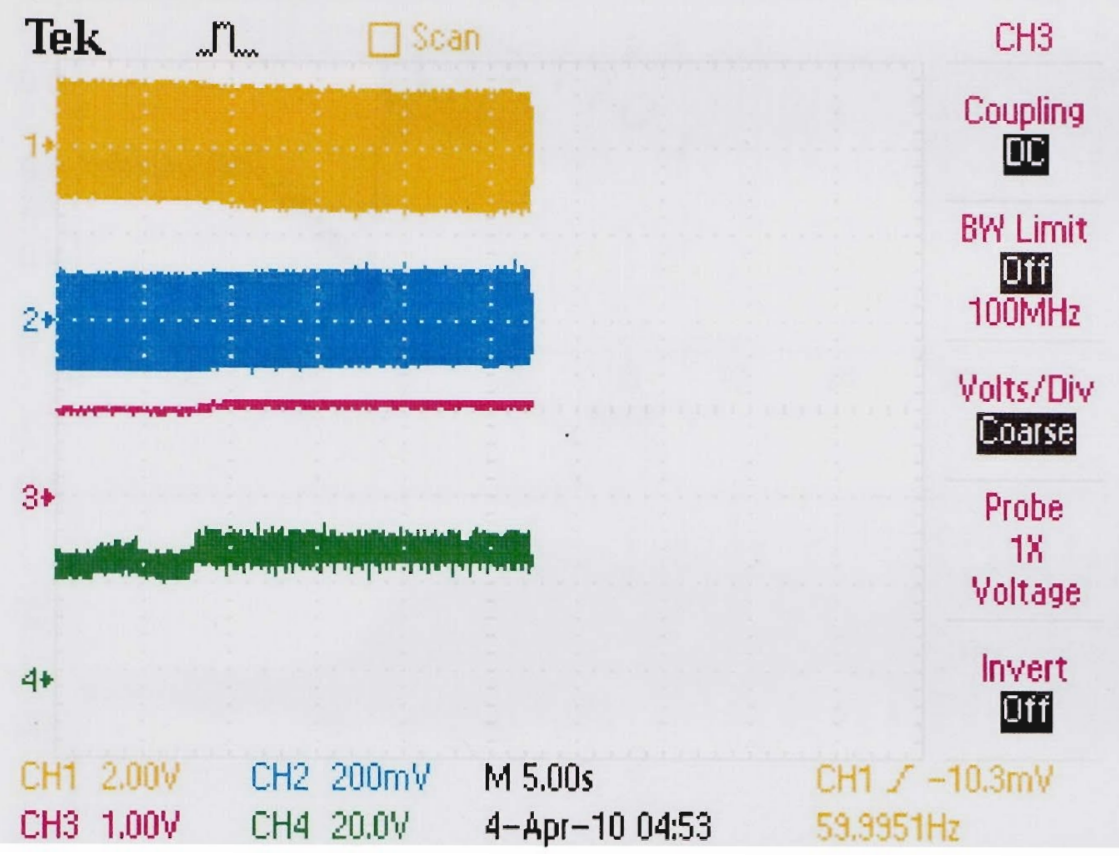

Figure 7.1-6 Load Bus and DC Bus

\subsubsection{Analysis}

The real time experimental data was also recorded to analyze the results in details and to present perspective of the experiment to display the power sharing between various components in the system, verify RMS values and observe any other special phenomena. The Mat lab file used for reading the data from the real time dSPACE values is provided in Appendix E.

The RMS voltage and current waveforms indicated that the variation is within the permissible range and also the figure displaying the power at each bus specifies the reduction in the amount of power tapped from the conventional generator. It should be noted that the DC bus power is the total power input to the 3 GTI's connected as a wye and the other power values are per phase. 

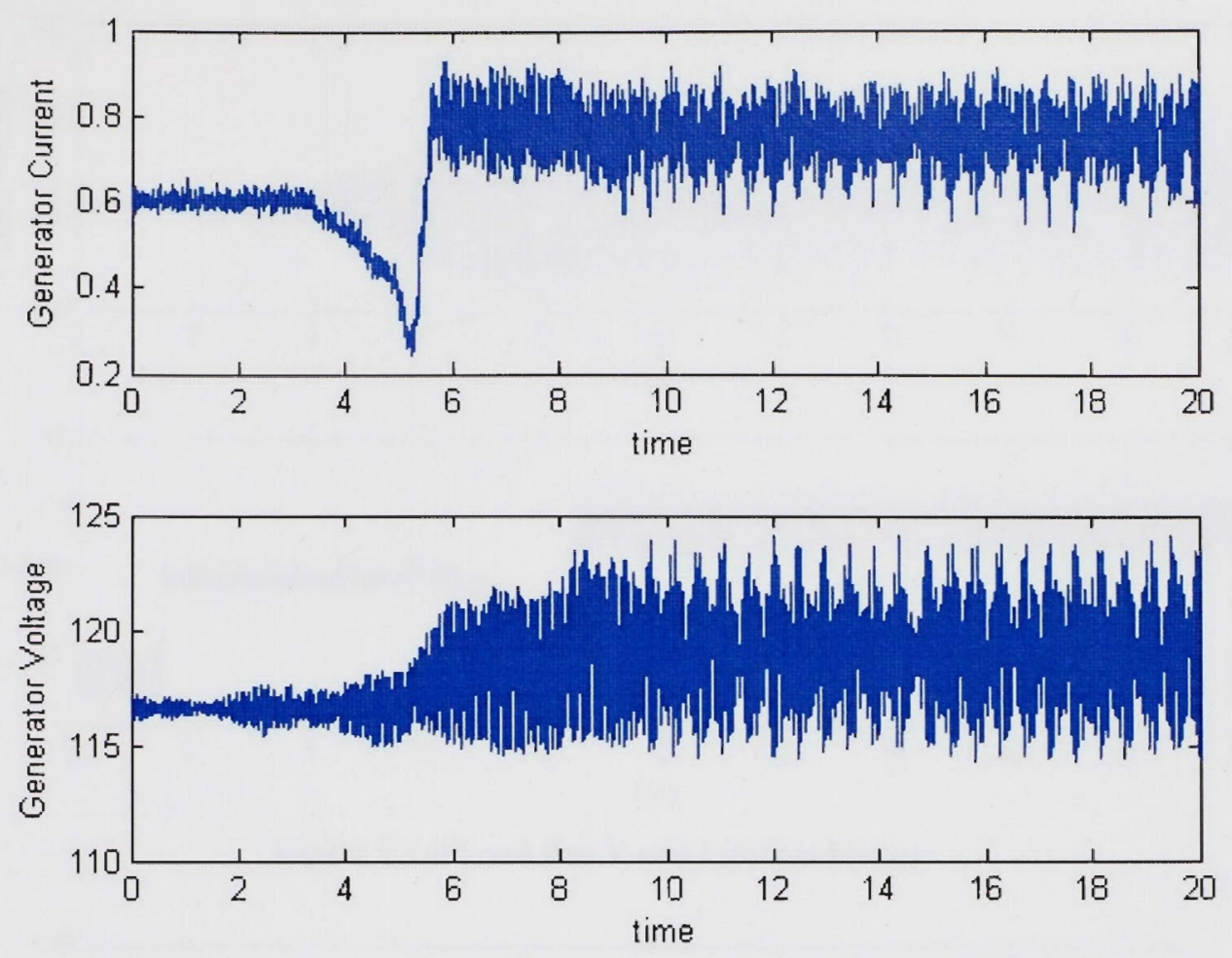

Figure 7.1-7 Gen V and I RMS (Single Phase)
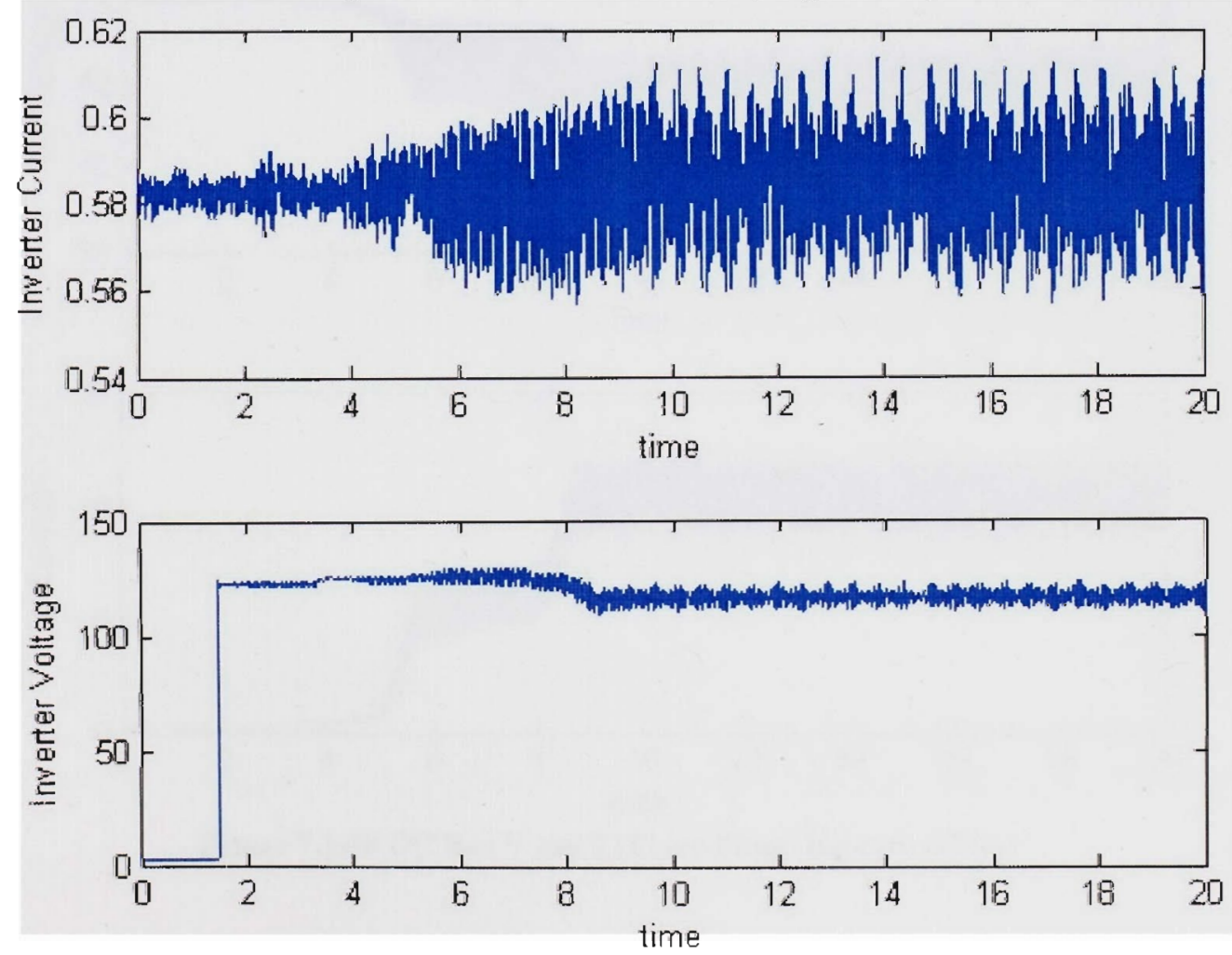

Figure 7.1-8 GTI V and I RMS (Single Phase) 

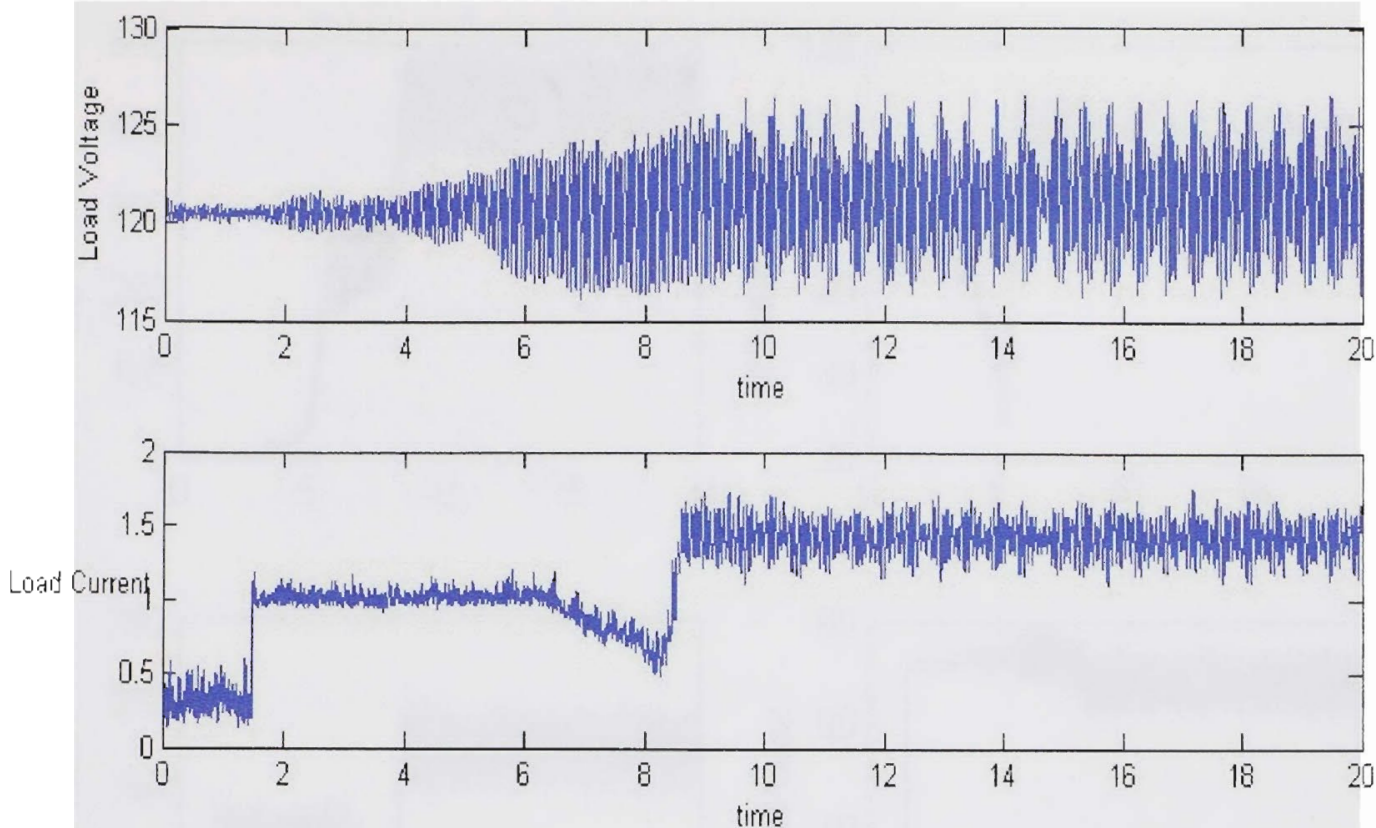

Figure 7.1-9 Load Bus V and I (Single Phase)
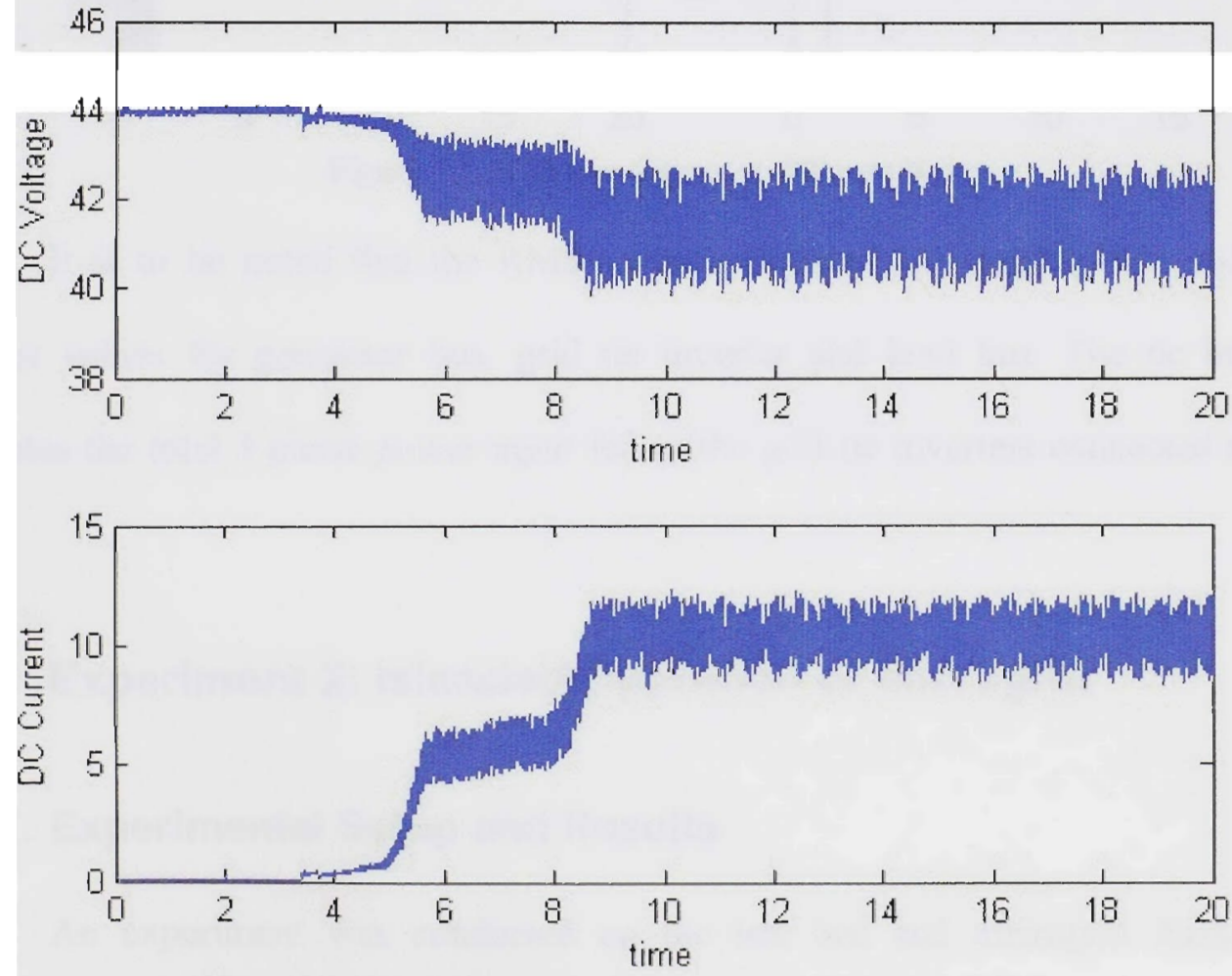

Figure 7.1-10 DC Bus V and I (Three Phase Input to GTI's) 

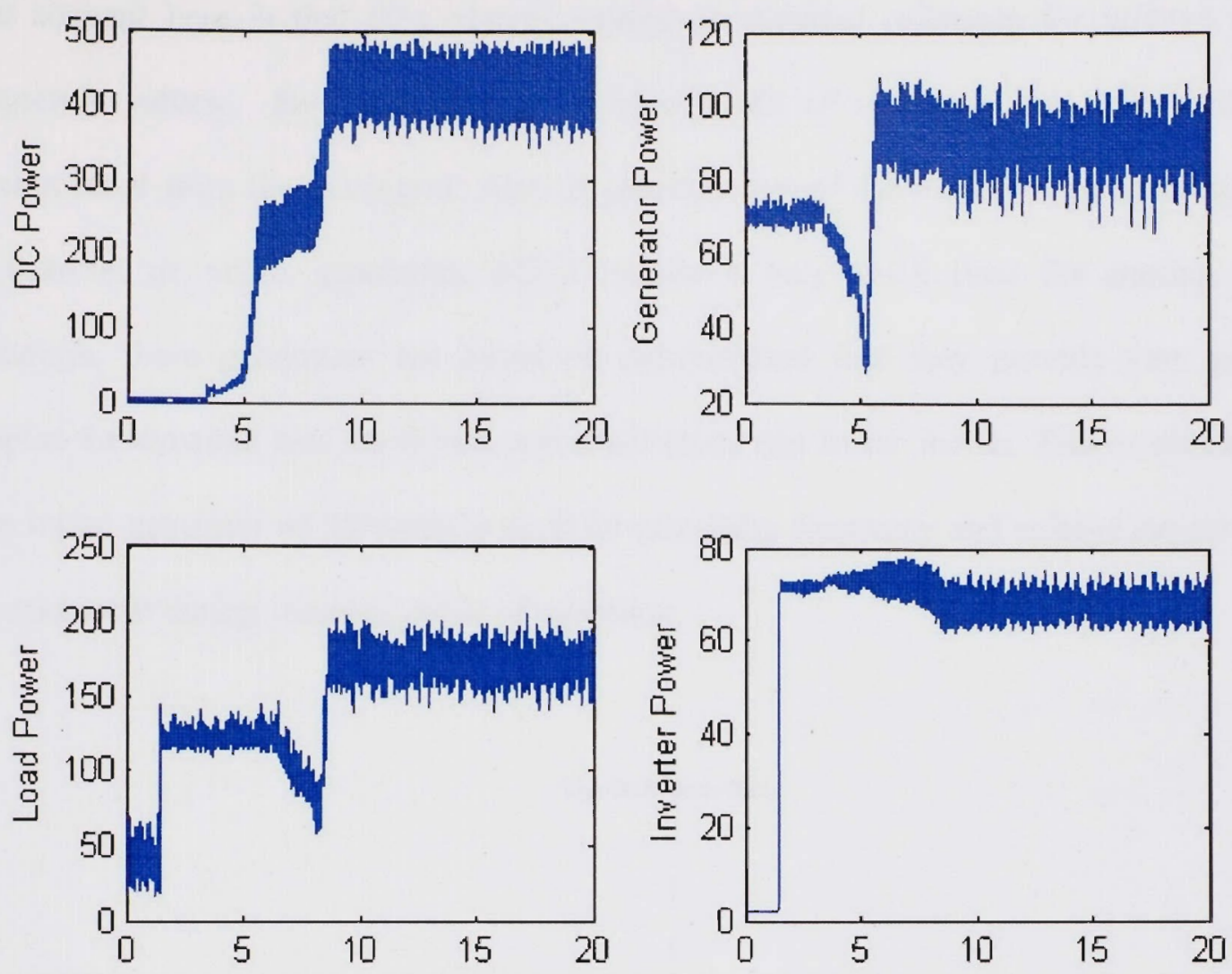

Figure 7.1-11 Power Values at different Buses

It is to be noted that the RMS values presented are single phase voltage and current values for generator bus, grid tie inverter and load bus. The dc bus power indicates the total 3-phase power input fed to the grid tie inverters connected as a wye.

\subsection{Experiment 2: Islanded Operation of Microgrid}

\subsubsection{Experimental Setup and Results}

An experiment was conducted on the test bed and microgrid framework to observe the capability of the microgrid in islanding mode. The present configuration of the microgrid deploys grid tie inverters which can work only in grid connected mode. 
The concept here is that they always require an external reference for voltage and frequency values. However, during islanded mode of operation the main grid is disconnected from the microgrid. Also in general most of the microgrid models utilize combustion or micro generators which require a very small time for starting up. Although, these generators are based on conventional fuel they provide very good support for dynamic load variations and small faults due to the inertia. This combustion cum micro generator of 250 watts is used for providing frequency and voltage support to the microgrid during islanded mode of operation.

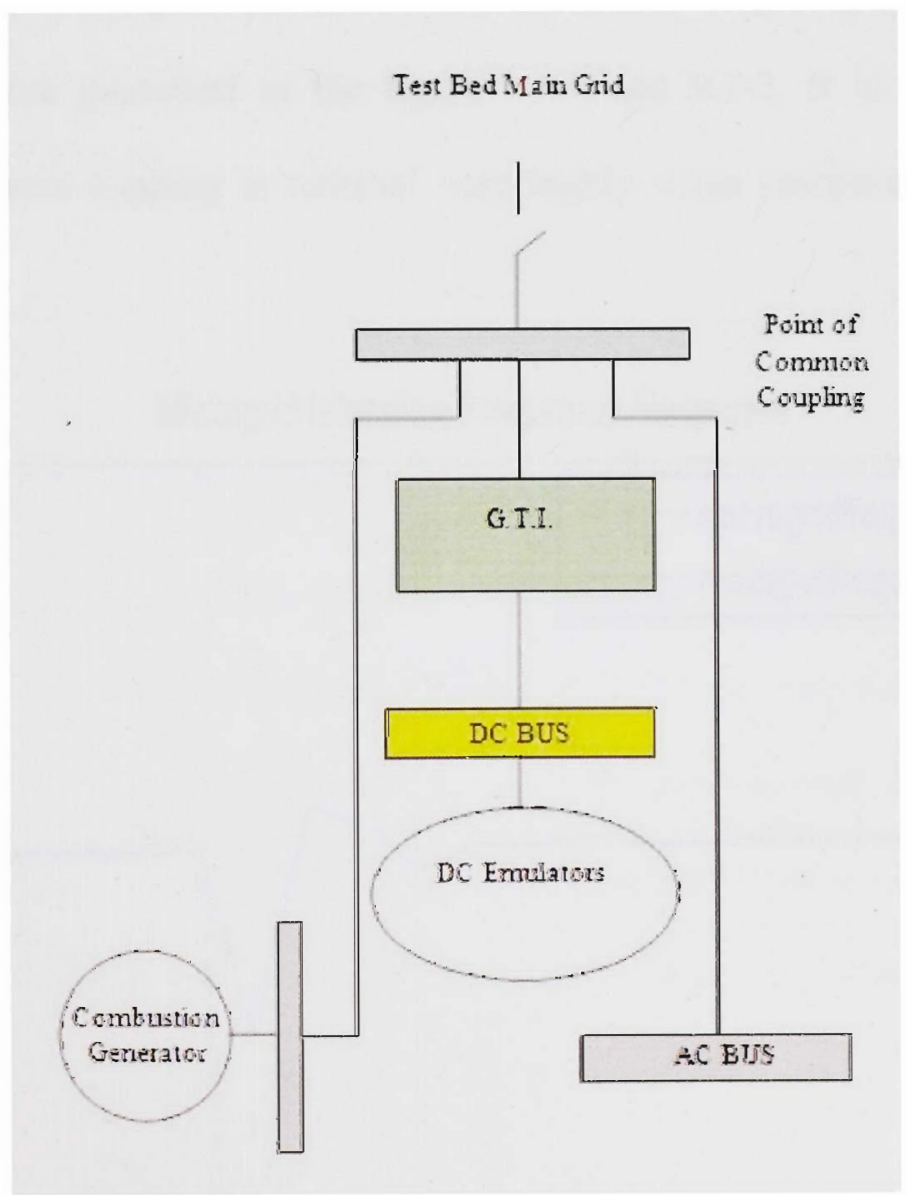

Figure 7.2-1 Islanded Operation Experiment 
As soon as the microgrid enters the islanded mode, the combustion generator turns on to provide voltage and frequency support to the microgrid. At the first instance the fuzzy logic controller is not turned on and the microgrid has the common problem of nuisance tripping. The problem of nuisance tripping is common a challenge for present scenario where the Grid Tie Inverter trips frequently during dynamic load variations due to changes in frequency and voltages references considering it to be a fault. It generally occurs in Grid Tie Inverters without internal reference capability.

During second instance of this experiment the fuzzy logic controller is turned on and is used for controlling the dynamic variations in the frequency and voltage values. The waveforms are presented in the figure 9.1-1 and 9.1-2. It is observed that the problem of nuisance tripping is reduced very highly when compared to the previous situation.

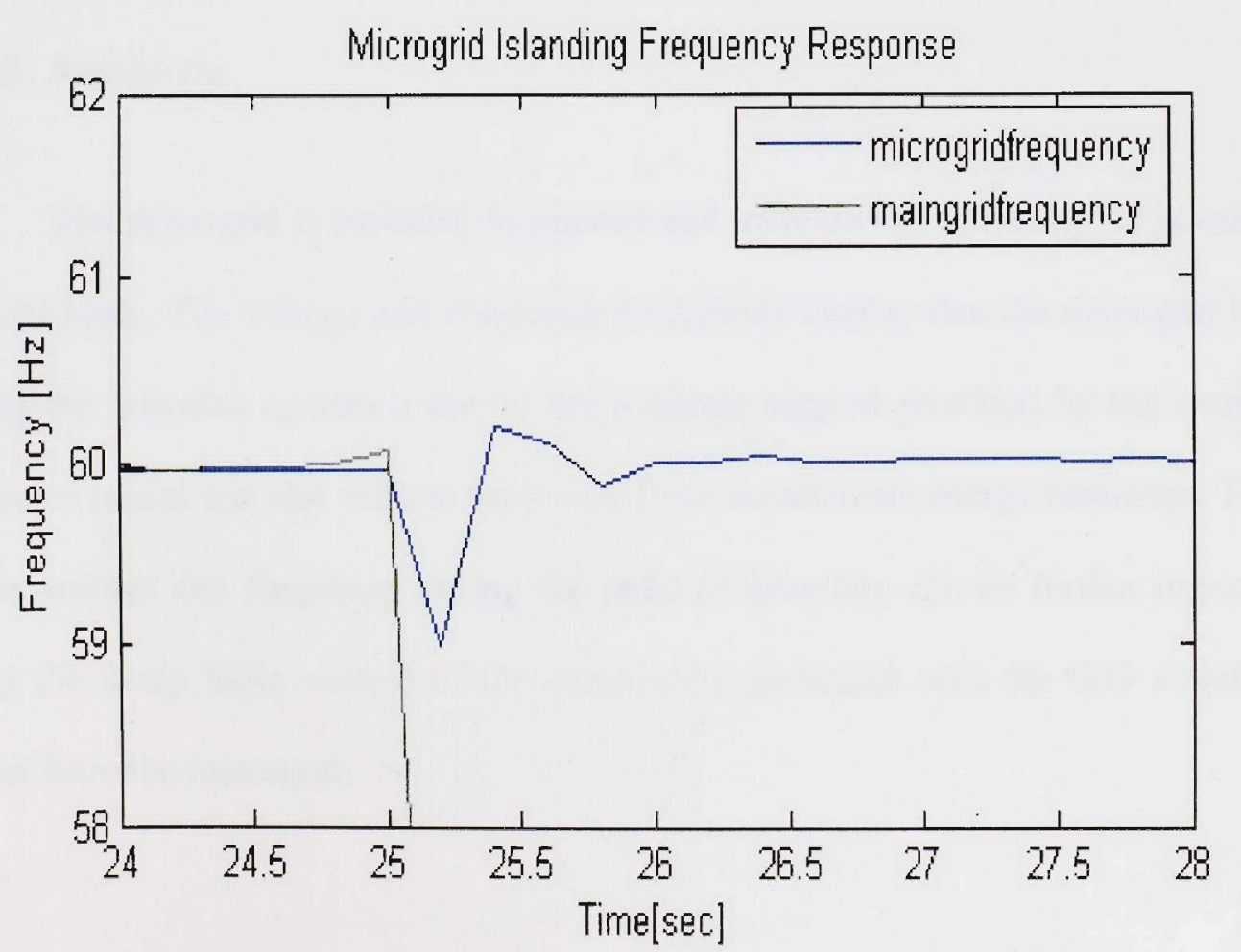


Figure 7.2-2 Frequency Response of Microgrid in Islanded mode

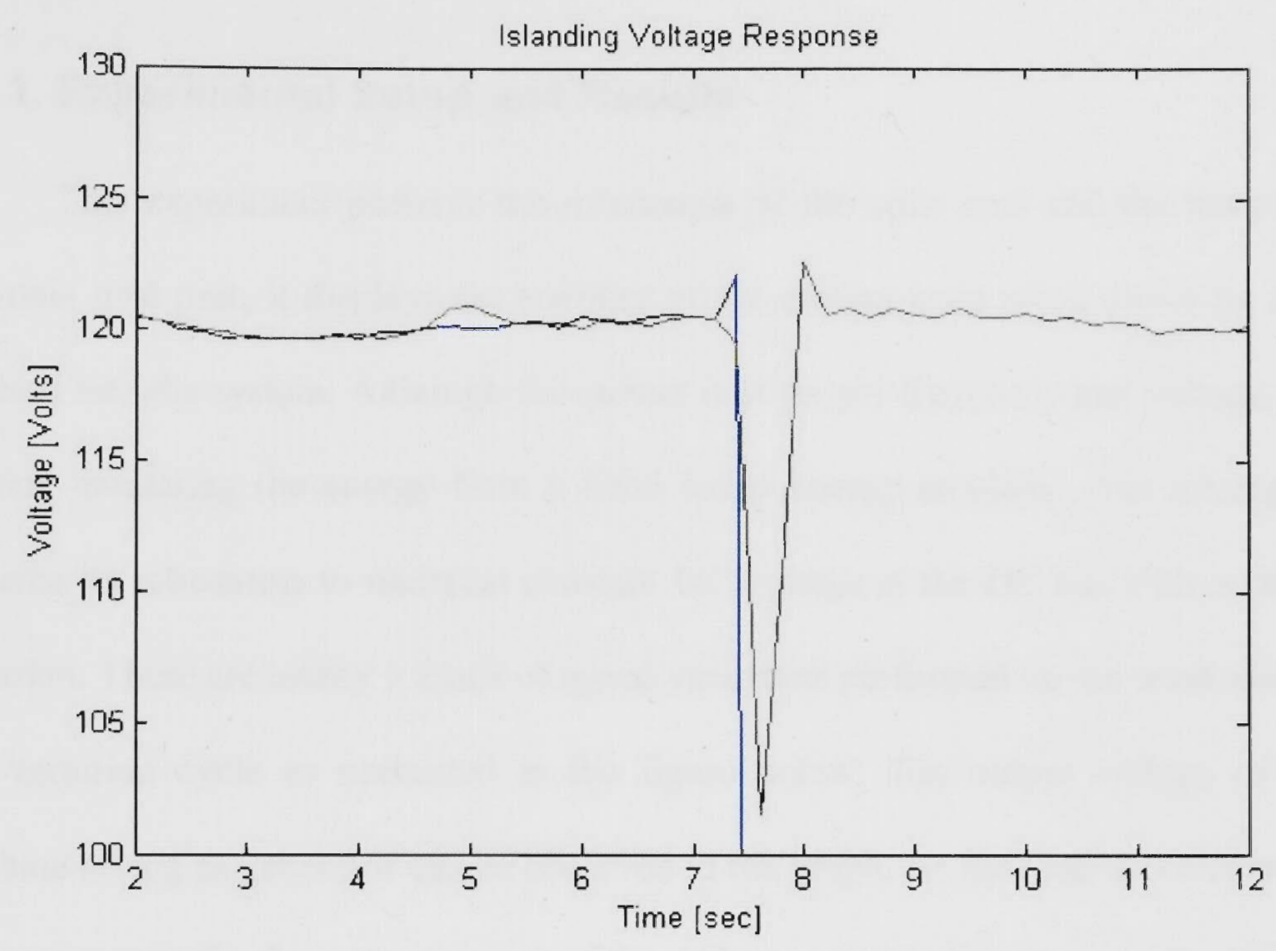

Figure 7.2-2 Voltage Response of Microgrid in Islanded mode

\subsubsection{Analysis}

The microgrid is modeled to operate and increase the reliability of power to the internal loads. The voltage and frequency waveforms display that the microgrid is stable during the islanded operation due to the dynamic support provided by the combustion generator model and also utilizes the power from the alternate energy resources. The drop in the voltage and frequency during the point of islanding can be further improved by using the fuzzy logic control of the combustion generator with the GTI which draws power from the microgrid. 


\subsection{Experiment 3: Wind Energy Integration to Test Bed}

\subsubsection{Experimental Setup and Results}

This experiment presents the robustness of the main grid and the microgrid, on the main grid part; it displays the stability of the system even when pulsating power is injected into the system. Although this power is at proper frequency and voltage, it keeps varying emulating the energy from a wind based energy emulator. The microgrid also presents its robustness to maintain constant DC voltage at the DC bus with a very small variation. There are totally 5 kinds of speed variations performed on the wind emulator in one variation cycle as presented in the figure below. The output voltage of the AC machine acting as generator can be observed in the graph. As the load is not connected to the system initially the current output of the AC generator is almost zero. The DC voltage output of the Buck converter is maintained at a constant Value.

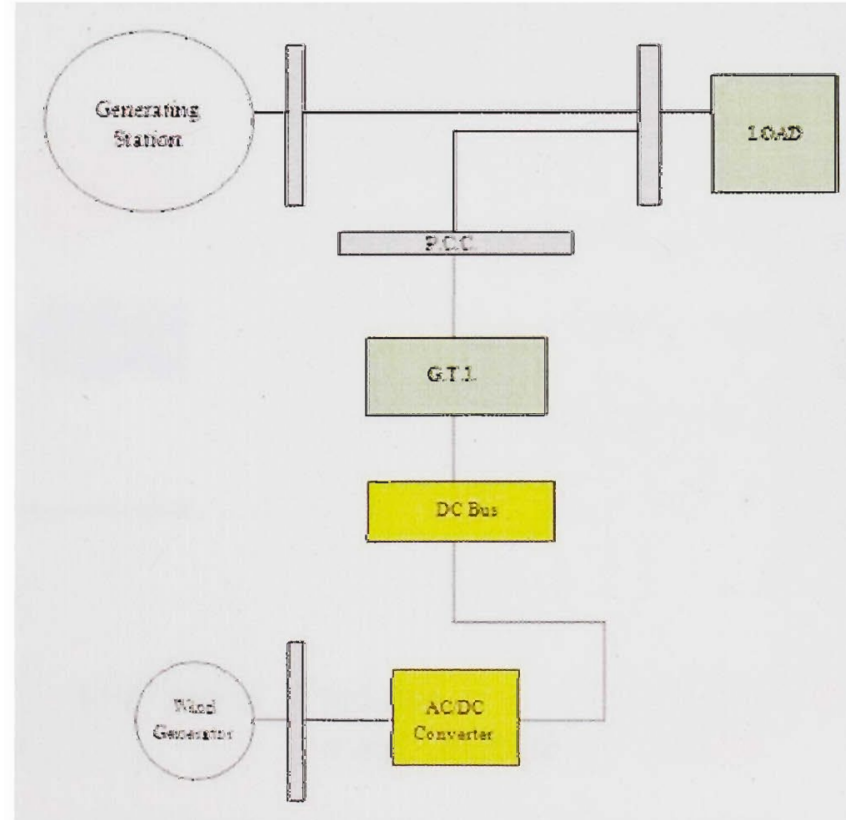

Figure 7.3-1 Setup for Wind Energy injection into test bed 
The variation of $\mathrm{AC}$ inpul to the $\mathrm{AC}-\mathrm{DC}$ buck converter, $\mathrm{DC}$ bus and Inverter input and output are displayed in the results in figures 7.3-2 and 7.3-3.

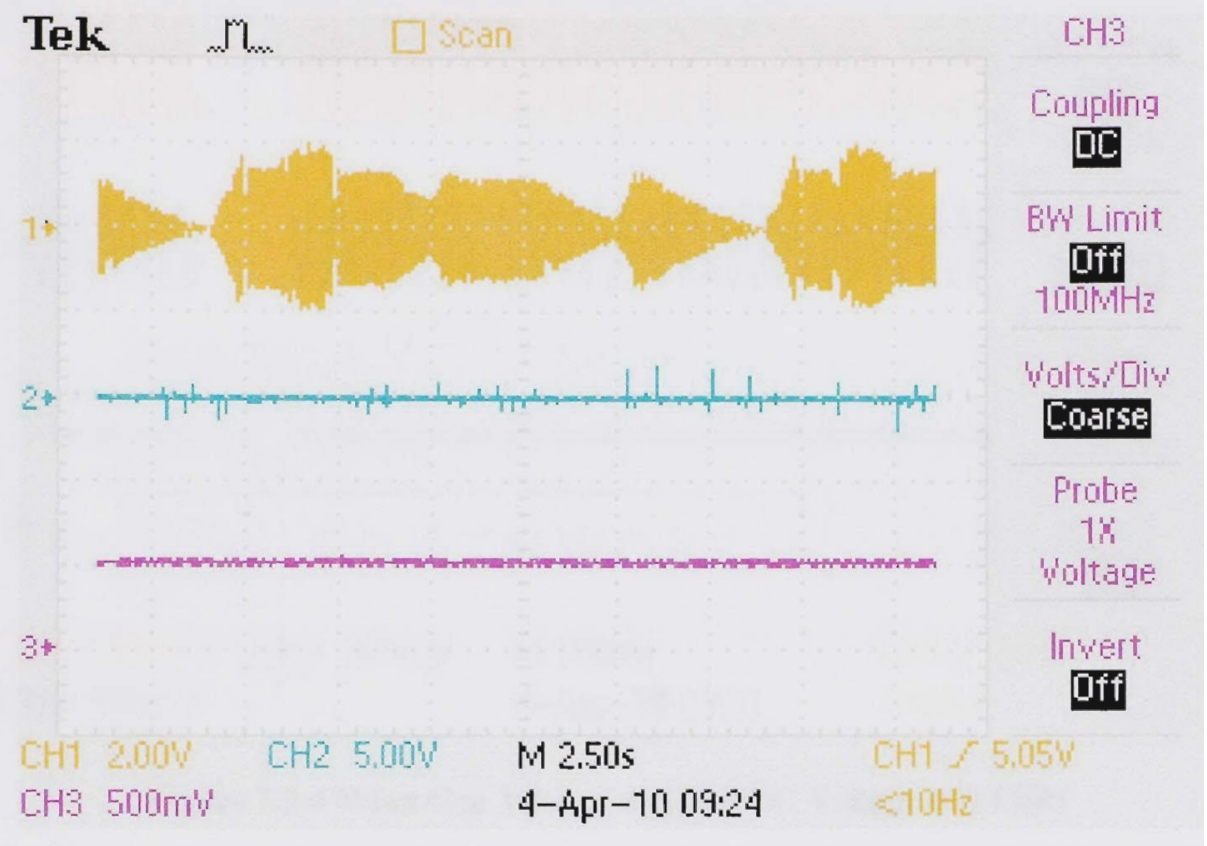

Figure 7.3-2 Wind Gen Voltage

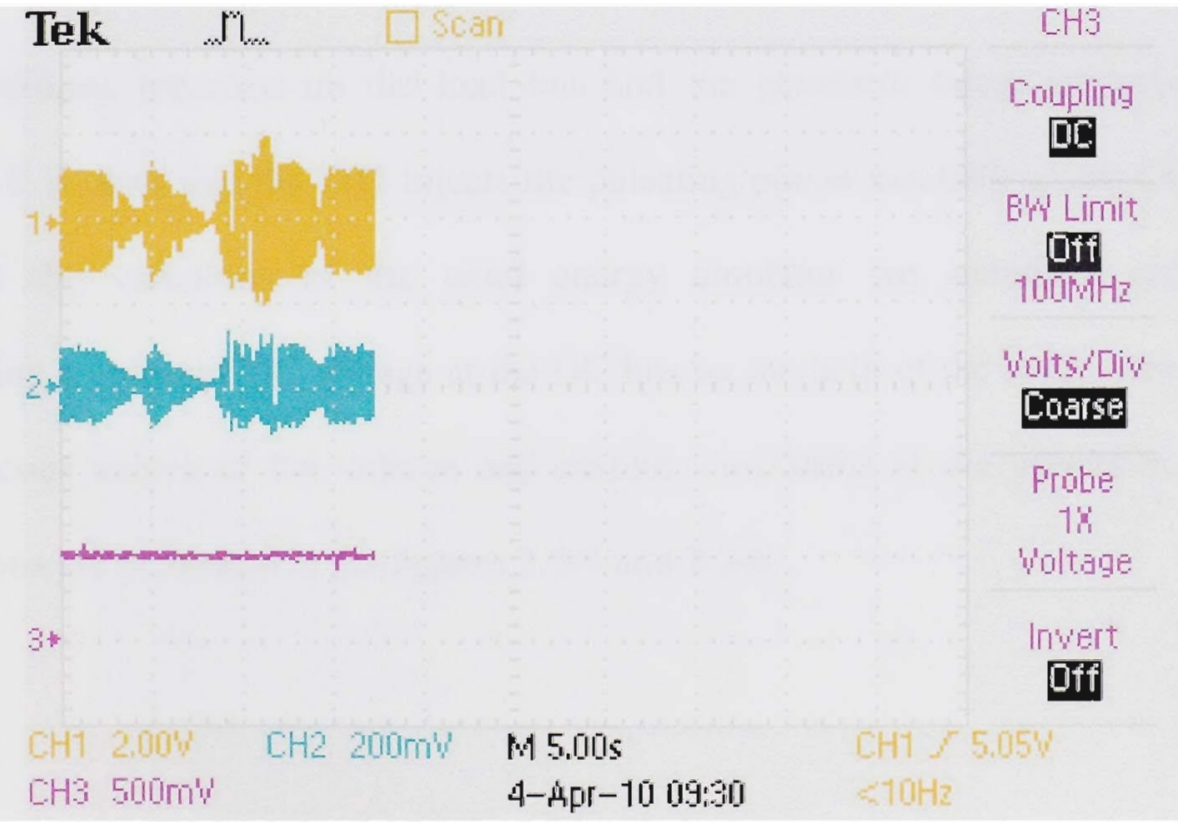

Figure 7.3-3 Wind Gen Voltage and Current at GTI Load 


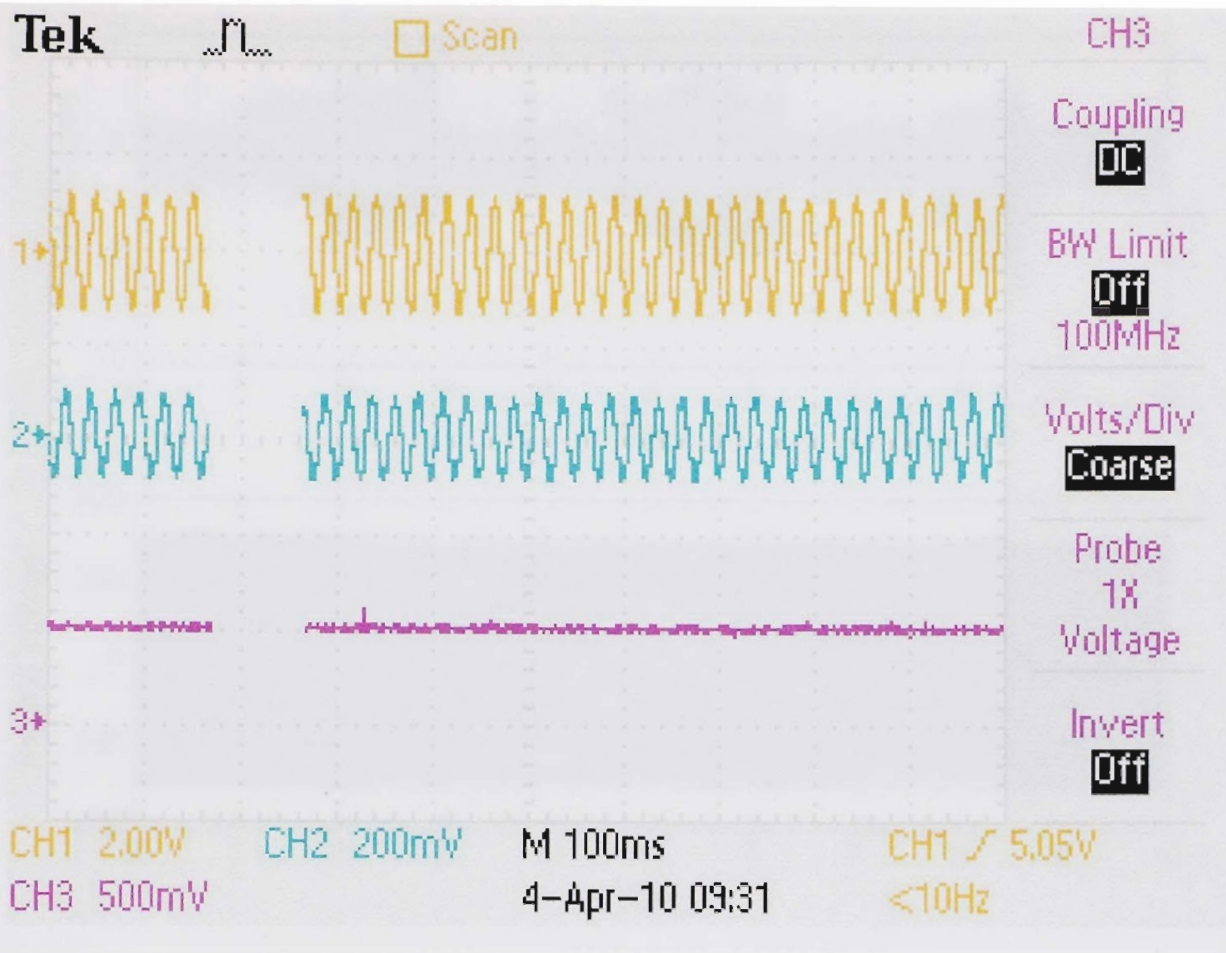

Figure 7.3-4 Wind Gen V/I and rectified DC Voltage (GTI I/P)

\subsubsection{Analysis of Results}

The waveforms recorded on the load bus and the generator buses are used for this analysis. It is clear that the GTI injects the pulsating power available at the DC bus and also that the variations of the wind energy emulator are stabilized properly by maintaining a constant DC voltage at the DC bus by the help of the buck converter. The instantaneous values of the voltage and current waveforms at the generator, load and inverter bus are presented in the figures 7.3-5 and 7.3-6. 

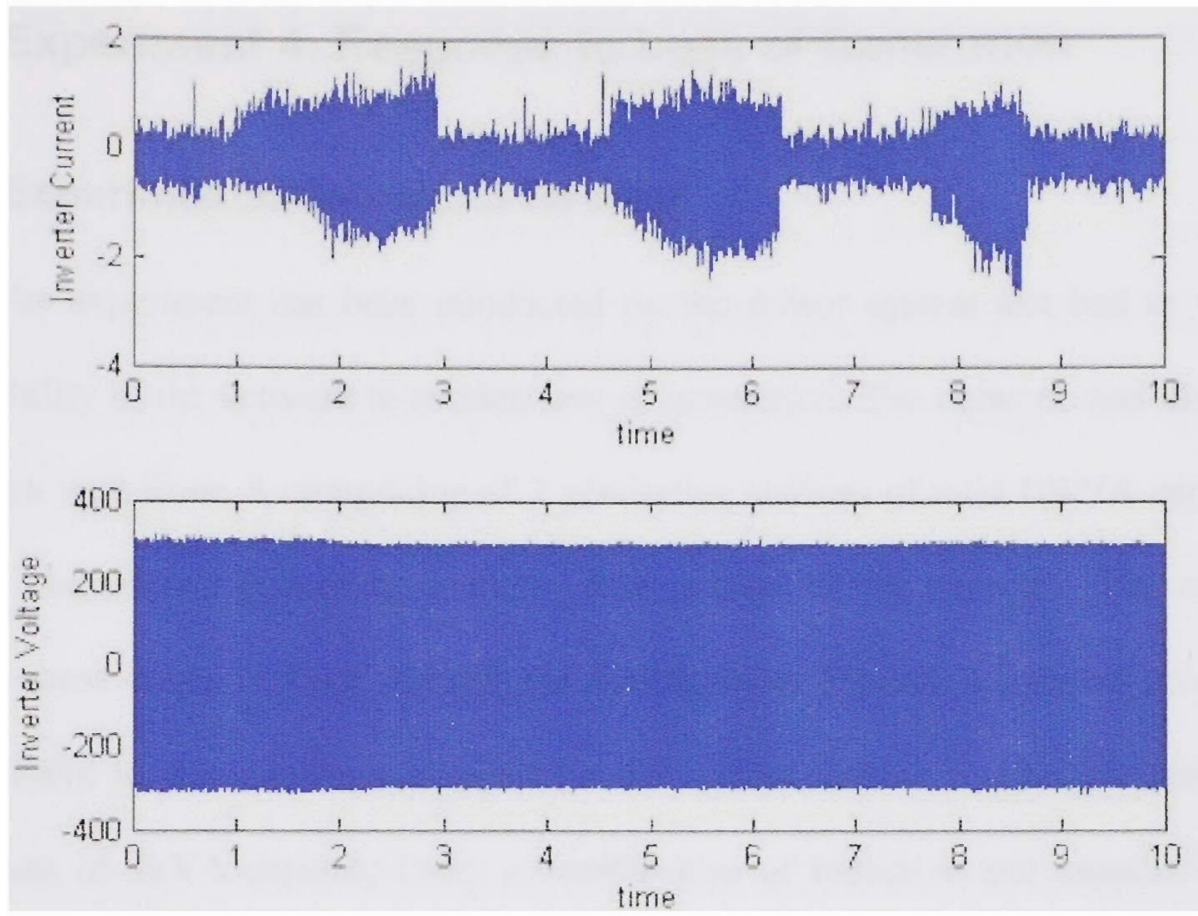

Figure 7.3-5 GTI V/I with Wind Input

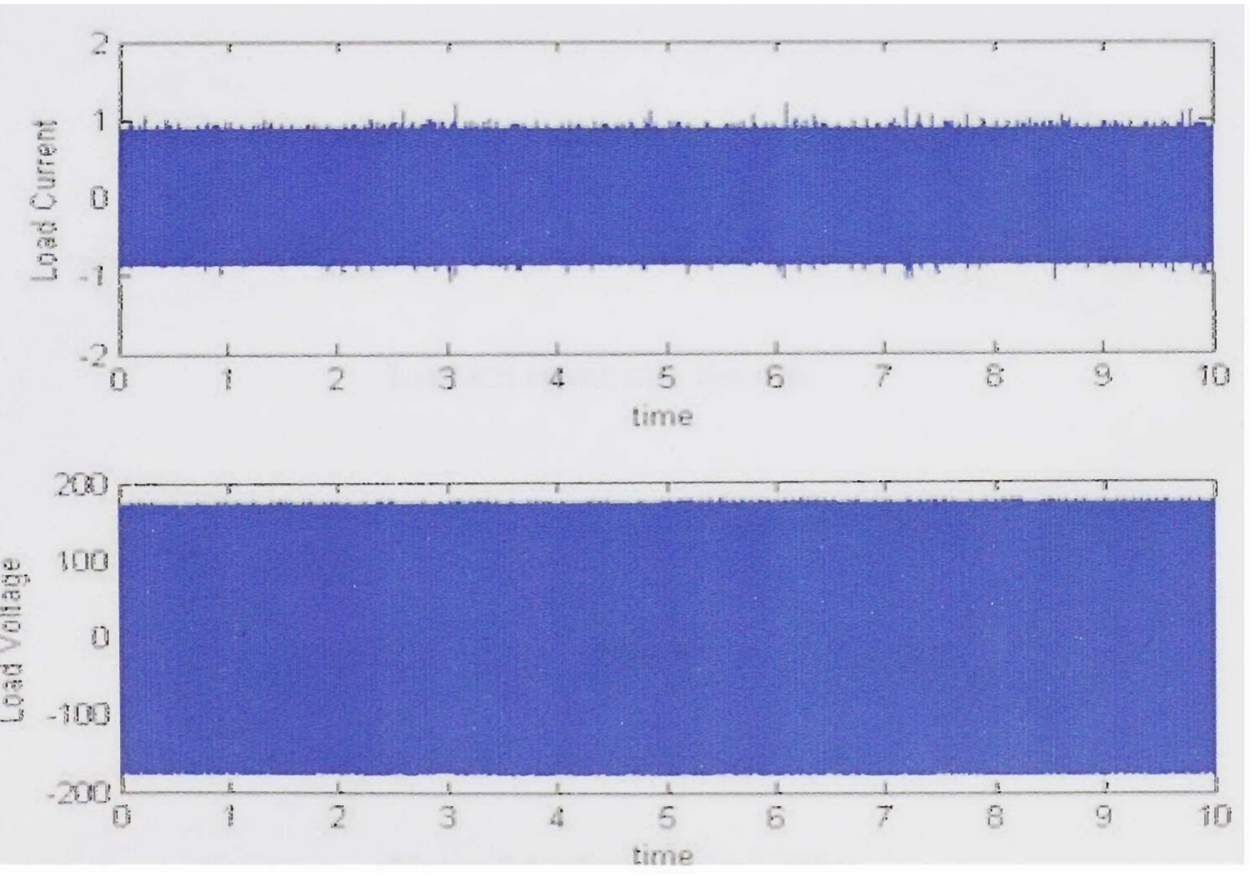

Figure 7.3-6 Load Bus Response on Wind Source Input 


\subsection{Experiment 4: Response to Loss of Generation}

\subsubsection{Experimental Setup and Results}

An experiment has been conducted on the power system test bed to verify the sustainability of the network to sudden loss of generation. The network was divided into two zones with Zone A comprising of 2 generating stations of total 10kVA capacity and Zone B comprising generating stations with a total 8kVA capacity. The connection between these zones is made through the Synchronizer Module 3 (labeled accordingly).

The different load emulators connected on the system include 5 inductive and resistive load boxes of $1 \mathrm{kVA}$ capacity each, a combination of inductive and capacitive load of 4.4kVA and three 250VA 3 Phase induction machines. The total load on the system is summed up to be around 10kVA.

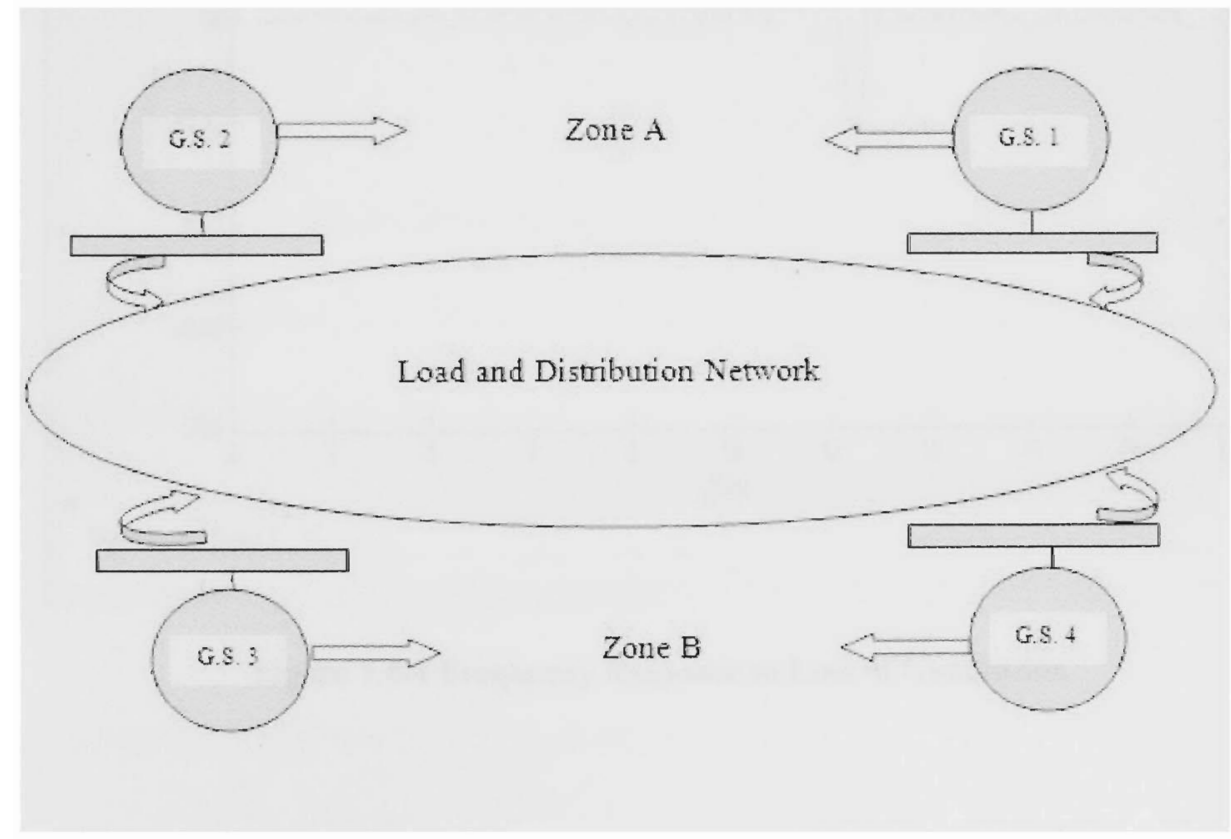

Figure 7.4-1 Loss of Generation

Zone 2 is disconnected by operating on the Labview GUI at computer system 3.

As zone 2 is disconnected a part of the load amounting to a total value of $2 \mathrm{kVA}$ is also 
Zone 2 is disconnected by operating on the Labview GUI at computer system 3. As zone 2 is disconnected a part of the load amounting to a total value of $2 \mathrm{kVA}$ is also disconnected from the system. A virtual instrument using labview is developed to record the voltage and frequency values to an excel sheet as a .lvm file. These values can be used for plotting waveforms to observe the response of the system. The frequency and voltage waveforms are plotted in figures 7.4-1 and 7.4-2.

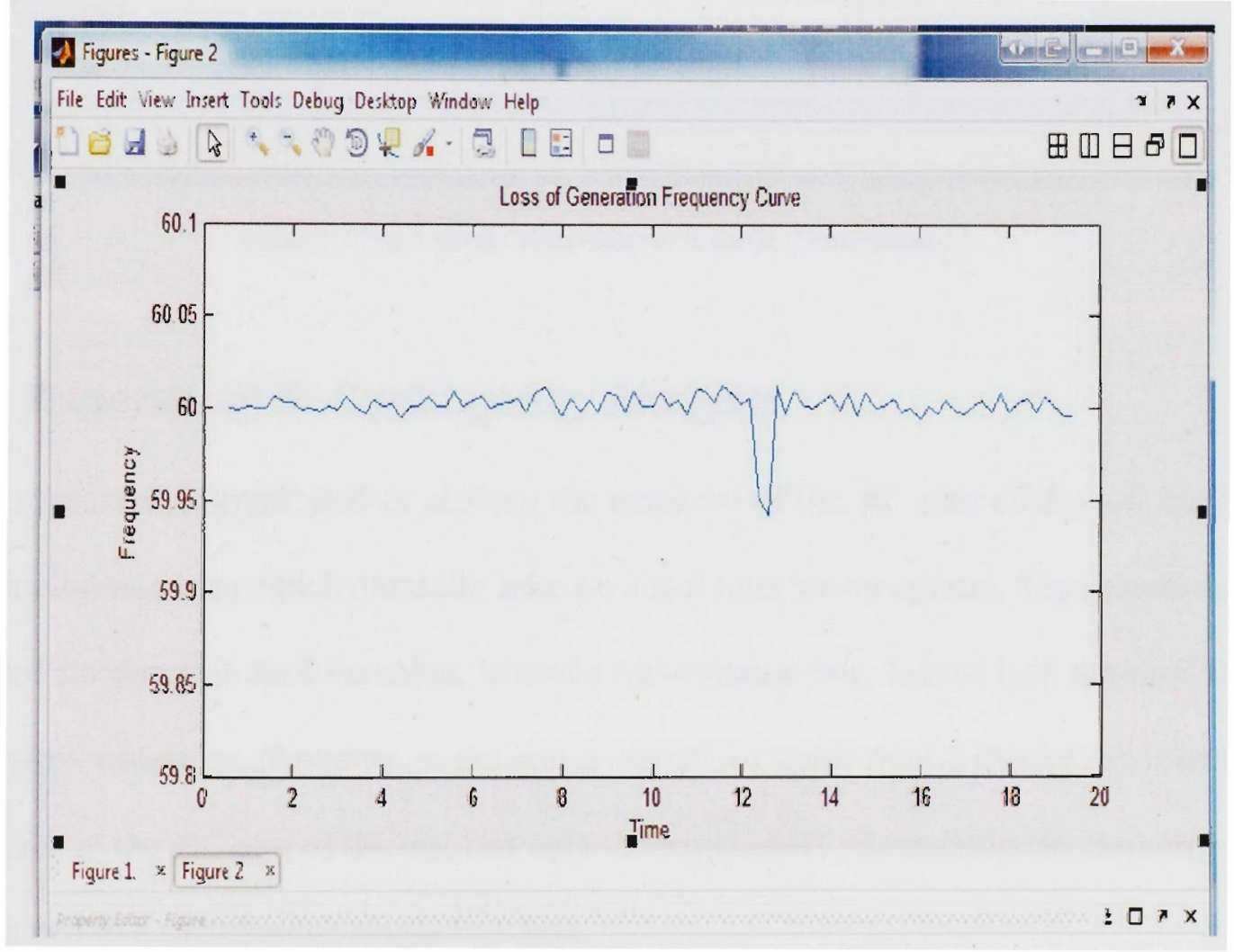

Figure 7.4-1 Frequency Response to Loss of Generation 


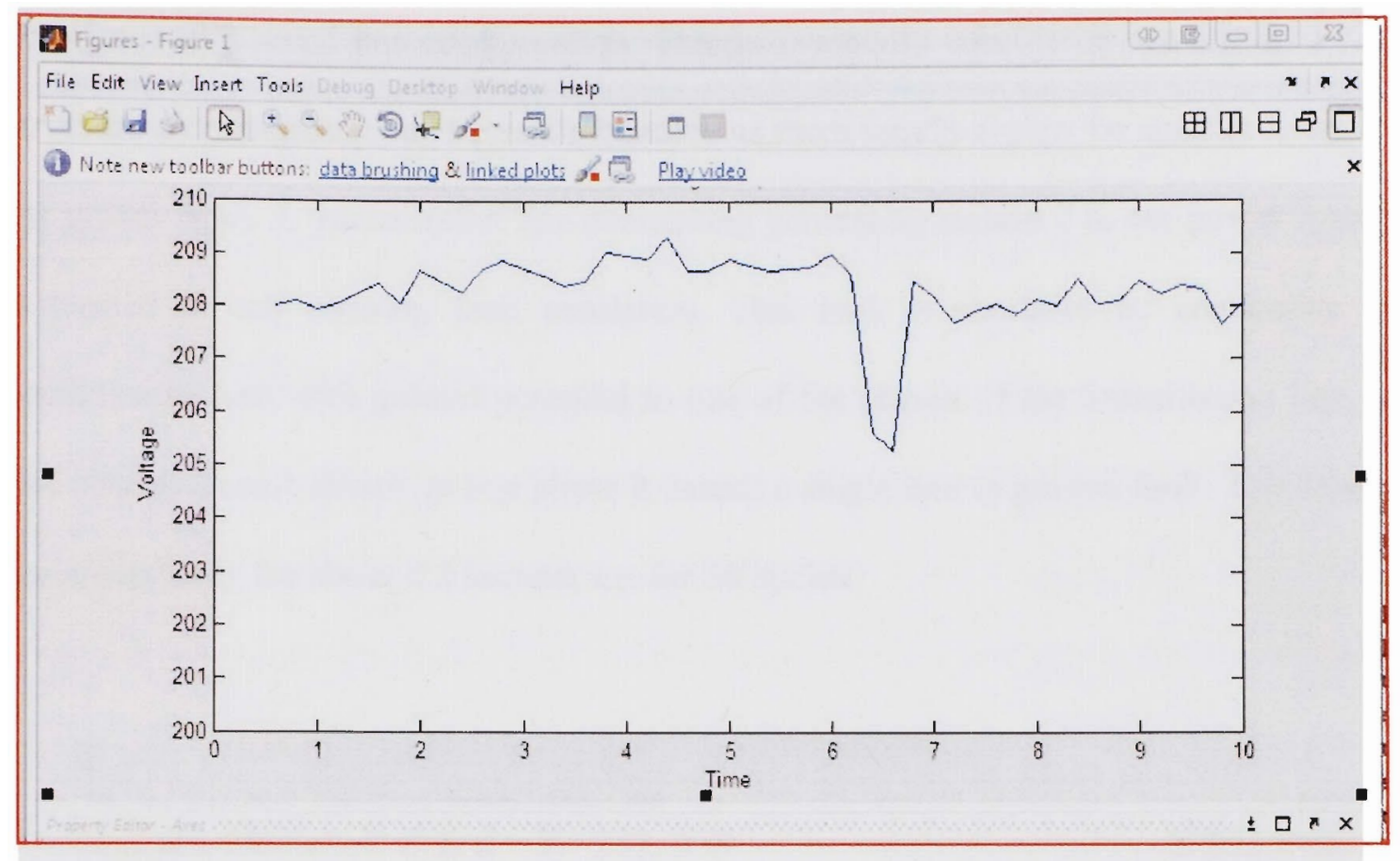

Figure 7.4-2 Voltage Response to Loss of Generation

\subsection{Experiment 5: Contingency Analysis}

This experiment is conducted to analyze the response of the AC part of the test bed to various contingencies which normally arise on a real time power system. The system was analyzed for dynamic load variation, loss of a transmission line, loss of load network and a few other variations. However, as the system remained stable during these experimental exposures to the $\mathrm{AC}$ part of the test bed only the experiment of verifying the response of the test bed to a self clearing single line fault.

\subsubsection{Experimental Setup and Results}

The power system network is also analyzed for a contingency response to determine its effectiveness. For the first contingency, the power system test bed is 
connected in a serial bus configuration. This is especially considered because in a ring structure a contingency can be easily handled as there would always be another route for the power flow. A transmission line connecting generating station 2 to the power system is treated to self clearing fault emulation. This fault is emulated by connecting an oscilloscope lead with ground potential to one of the phases of the transmission line, as this creates a short circuit on one phase it causes a single line to ground fault. This lead is connected only for about 0.5 second, i.e. for 30 cycles.

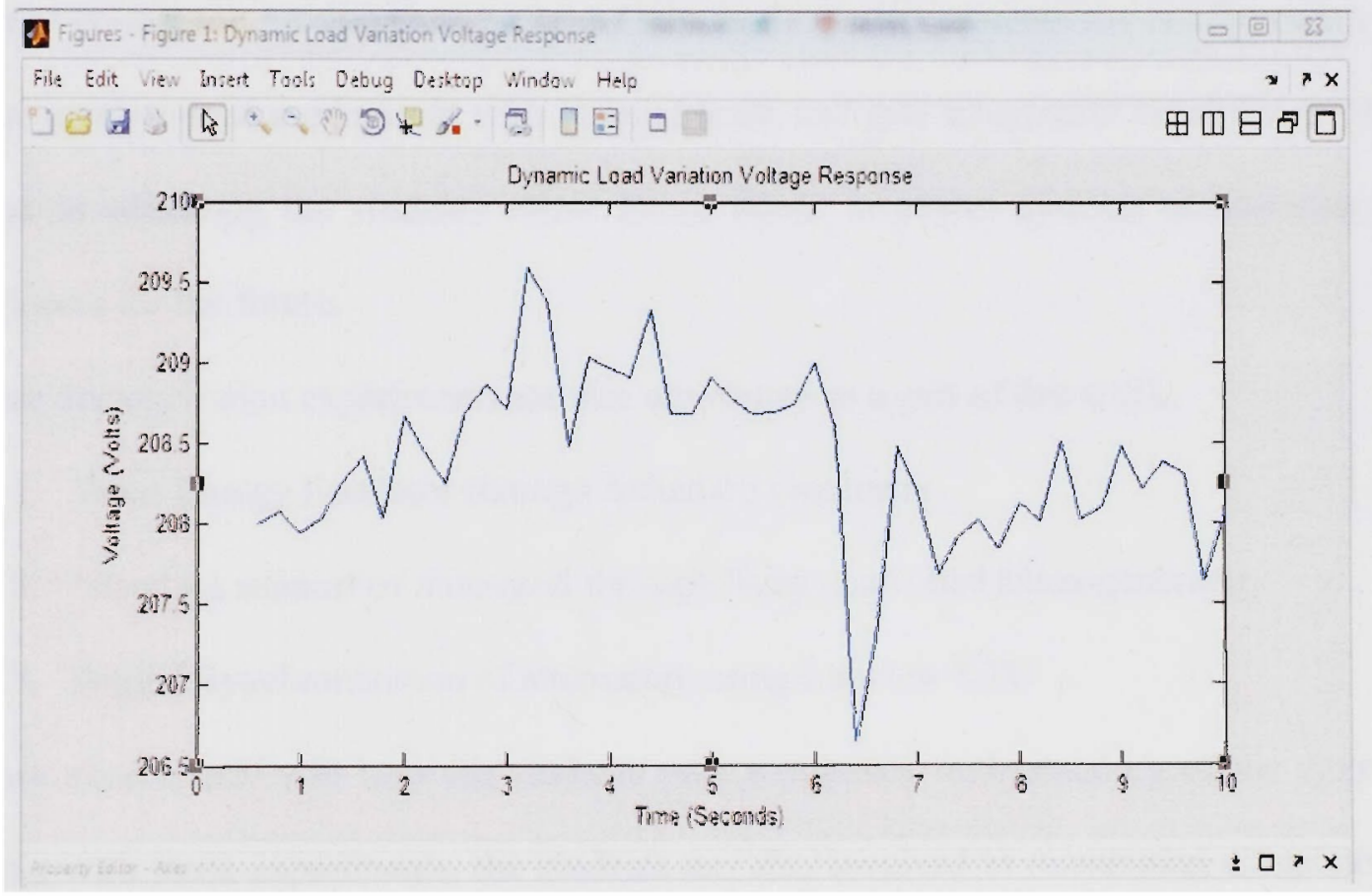

Figure 7.5-1 Frequency Response for self clearing fault 


\section{PRACTICAL USE AND SCALING ISSUES}

This chapter presents the practical use of the test bed by examining and presenting research and educational applications. It also presents the scaling issues of this system.

\subsubsection{Educational Impact}

The test bed is developed for demonstrating the power system operation and control techniques to the under graduate and graduate students. It acts as an effective tool for demonstrating the sustainable energy resources operation through the various alternate energy emulators developed as a part of this work. It also presents the configuration of these emulators as a microgrid with internal loads and grid integration capabilities. This helps in educating the students about future issues in power systems and to educate engineers for the future.

Three demonstration experiments are also developed as a part of this work.

1. Wind Energy Emulator through Induction Generator.

2. Islanding support of microgrid through fuzzy controlled Microgenerator

3. Digital Synchronization of alternators using Labview RTU

These experiments will help the students gain a practical understanding of the system Along with these experiments, the students are also involved in developing component: for the test bed providing adequate knowledge to gain hands on experience. A few mor demonstration experiments can be added to increase the test bed perform as a educationa laboratory for power systems. The details of experiments are provided in the appendix. 


\subsubsection{Scaling and System Description}

The power system test bed contains different equipments and components such as generating stations with electric drives on the prime mover control, protective devices (relays, fuses, switch breakers etc). The test bed was originally modeled and scale up to IEEE 15 bus system and is a three phase system operating at $25 \mathrm{kVA}$ generation capacity from conventional sources at two low voltage level of $120 \mathrm{~V}$ phase and high voltage level of $240 \mathrm{~V}$ phase at $60 \mathrm{~Hz} \mathrm{AC}$ frequency. The generation and storage capacity from the alternate energy resources is $10 \mathrm{kVA}$ and hence the present operating capacity of the system is $35 \mathrm{kVA}$. The DC bus operates at two voltage levels $42 \mathrm{~V}$ and $100 \mathrm{~V}$ for interconnection with appropriate loads.

The sensor and monitoring system is scaled down by using the secondary side impedance of the VT's and CT's on the AC system and by the transducer ratio on the DC zone in the microgrid. This allows rapid scaling of the system component such as transmission lines and buses. The scale here is referred to the secondary side of the data acquisition devices. 


\section{DISCUSSION OF OVERALL RESEARCH RESULTS}

The power system and distributed generation test bed is a combination of several subsystems, the emulation of this test bed cannot be represented without acknowledging the alternate resource emulators and implementation of various parts of the conventional power system on the test bed. In this thesis analysis of various subsystems in performed.

Alternate resources are implemented by analyzing the techniques to represent an actual system by simulating the characteristics of a real time model and developing a emulator representing the real time characteristics. The responses of real time alternate emulators and modeling of hardware emulators to represent the real time characteristics is presented in chapter 2 . Two wind energy emulators representing high voltage and low voltage farms are presented in chapter 2 along with a fuzzy logic block developed for frequency and voltage regulation in hardware in the loop combustion generator designed for islanding support in a microgrid.

Generating station emulators representing power plants in an AC system are presented for their response similar to an actual system. Transmission line models and bus bar models for transmission and distribution system are presented with a DAQ system on the test bed in chapter 3. Chapter 3 also presents the block diagram designed to represent the test bed and the simulation of the test bed in MATLAB Simulink for verifying the response of interconnection of the AC system.

The developed components for the AC system are interconnected to implement the conventional AC system of the test bed. AVR response and governor control through the drives of the induction machine acting as a prime mover are presented. Chapter 4 also 
presents the synchronization module and other applications built in LabView for control and monitoring of the AC system.

The interconnection of the alternate source emulators presented in chapter 2 is presented to emulate a microgrid model with internal loads and generation in chapter 5 . Virtual Instrument is developed in LabView for monitoring and control of the microgrid enabling in data logging for energy exchange between the AC system and the microgrid. A module is also developed to force microgrid into islanding mode for experimental analysis. DC bus on the microgrid to distribute the power from alternate emulators and connect the internal DC loads is also presented in chapter 5.

Communication layer for control and data logging issues for stability studies are developed in chapter 6. VI's for implementing the communication using ModBus protocol for motor drives used as prime movers for generating stations are developed. Communication system using Shared Variable in LabView for inter-process and interplatform communication on the test bed is also developed and the SCADA system is presented emulating a central monitoring station. The VI's are also published as web page on the internet using the LabView web server for remote access and control especially designed for home application of microgrid.

Experimental analysis of the system is conducted by verifying the response of the system during the energy sharing between alternate resources and the $\mathrm{AC}$ power system. System is also evaluated for stability during injection of wind energy based pulsating power input to the AC system. The load and the generator voltage and current are monitored for this experiment. Another experiment is conducted to analyze and study the response on voltage and frequency on the load bus of an internal load in a microgrid 
during the state of islanding; this experiment also presents the advantages of the fast starting combustion generator developed in chapter 2. Two experiments are conducted to demonstrate the operation of the AC system as a real time power system with evaluation to loss of generation in a zone and also for a contingency.

The power system test bed is emulating an AC power system integrated with microgrid at different points in the system due to the integration of these subsystems. It acts as a platform to continue research and also for educational demonstration of future power systems with distributed generation and control using hardware in the loop based components.

Experiments are also devised in chapter 8, for improving the present education system in power system by accommodating the alternate energy resources and microgrid concepts. The basic power system operation is also explained in a better manner practically through the various applications developed for monitoring and control. This helps in creating better education facilities for future engineers with adequate information about the issues pertaining to the present and future power systems. 


\section{CONCLUSION}

In this work the state of the art for power system and microgrid integration is advanced by developing a test bed and emulating a microgrid with sustainable energy resource emulators.

Distributed generation resource emulators and their operation as a microgrid in synchronization with emulated power system test bed have been successfully demonstrated. The development process started with a thorough literature review and it was determined that the present research work stresses on microgrid component modeling but does not verify the issues related to integration by physical implementation.

The distributed generation resources developed as a part of this thesis successfully emulates characteristics of the real time systems. Synchronization of the microgrid with the power system was demonstrated successfully. The present work demonstrates a significant ability to verify the effects on the microgrid and power system. These alternate sources can be deployed to increase the power capability of the present system and increase its dependability and also improve the stability.

The experimental result on microgrid islanding presents that the techniques developed can be deployed for the future power systems with non conventional resources in load centers.

Experiments are then developed on the test bed for demonstration and educational purposes and the impact of the test bed for educational improvement is also presented. The overall research results of the work are also discussed. 


\section{FUTURE WORK}

The microgrid developed as a part of this work on the test bed represents all the concepts of alternate resources; however, the microgrid deploys grid tie inverters which can only inject power into the test bed as the grid tie inverters can work only on power injection model. Semikron module can be used to develop GTI which can draw power from the test bed for loads within the microgrid during shortage of power from the main grid.

DC bus deployed on the microgrid is used for connecting to regulated sources and distribution and it can be greatly improved by developing a DC distribution system which connects all the sources and loads on the DC side in the microgrid. The DC load centers can be improved further by connecting DC/AC converters for DC distribution system. This system can also help in integration of hybrid electric vehicles into the grid following a certain program for power sharing and power density management. Dynamic behavior of the microgrid can be improved further by using flywheel models and the motors on the generating stations in the test bed can be utilized for this purpose.

The load models developed for the test bed are manually variable for load changes. This can be further improved by emulating constant current, impedance and load models using inductance coils and by emulating various machine behaviors using the phase variable model.

In this work the SCADA system is developed to monitor the parameters of the syster at all the buses and transmission lines. This can be further developed as a PMU by logging the data in the real time and using it for online evaluation of the system and tc use the simulation data during any failures in the communication system. 


\section{REFERENCES}

[1] Chris Marnay, IEEE Member and Giri Venkatraman, IEEE Member "Microgrids in the Evolving Electricity Generation and Delivery Infrastructure" 2006.

[2] Huang Jiayi, Jiang Chuanwen, Xu Rong "A review on distributed energy resources and Microgrid", 14 May 2007 Renewable and Sustainable energy reviews Elsevier.

[3] F.D.Kanellos, A.I. Tsouchinkas, N.D.Hatziargyriou, Senior Member, IEEE "MicroGrid Simulation during Grid-Connected and Islanded Modes of Operation

[4] Robert H. Lasseter, Fellow, IEEE, Professor Emeritus, University of WisconsinMadison, "Microgrids and Distributed Generation".

[5] Paul Hines, Karthikeyan Balasubramaniam and Eduardo Cotilla Sanchez "Cascading failures in power grids" IEEE Potentials September/October 2009 Vol.28, No.5.

[6] B.Fardanesh "Future Trends in Power Systems control", IEEE Computer Applications in Power, 2002.

[7] Nikos Hatziargyriou and Goran Strbac "Microgrids-A possible future energy configuration", IEA Seminar on distributed generation: key issues, challenges, roles", Paris, March 2004.

[8] Robert H. Lasseter and Paolo Piagi "Microgrid - A conceptual Solution".

[9] O.A.Mohammed, N.Cristaldo and N.Abed "Secure Power Grid Infrastructure Simulation", Power Engineering Society, Tampa, June 2007.

[10] Nelson Cristaldo "Secure Power Grid Infrastructure and Test Bed Creation", Thesis, Masters in Electrical Engineering, F.I.U, August 2007.

[11] Thomas Ackermann, Goran Anderson and Lennart Soder "Distributed Generation: A definition", Electric Power Systems Research, Elsevier, Vol.57, Issue 3, $20^{\text {th }}$ April 2001.

[12] W. El-khattam and M.M.A. Salama "Distributed generation technologies, definition and benefits", Elsevier, $7^{\text {th }}$ May 2004.

[13] N. Hatziargyriou, H. Asano, R. Iravani, Reza, Marnay and Chris "Microgrids: An overview of ongoing research, development and demonstration projects", IEEE Power and Energy Society, Vol.5, July-August 2007.

[14] Chris Marnay, F. Javier Rubio and Afzal S. Siddiqui "Shape of the Microgrid", IEEE Winter Meeting Columbus, OH, 31 ${ }^{\text {ST }}$ January 2001. 
[15] Shashi B. Patra, Joydeep Mitra and Satish J. Ranade, Klipsch School of Electrical and Computer Engineering, New Mexico State University.

[16] Giuliana Marshall "Modelling of a Micro-grid System", Thesis for Bachelors in Electrical Engineering at The University of Newcastle, Australia, October, 2004.

[17] J.A. Pecas Lopes, C.L. Moreira, A.G. Madureira, F.O. Resende, X. Wu, N. Jayawarna, Y. Zhang, N. Jenkins, F. Kanellos and N. Hatziargyriou "Control Strategies for Microgrids Emergency Operation”, IEEE Xplore.

[18] J.A.Pecas Lopes, C.L. Moreira and F.O. Resende "Microgrids Black Start and Islanded Operation", $15^{\text {th }}$ PSCC, Liege, 22-26 August 2005.

[19] kojovic and R. Willogby "integration of distributed generation in a typical USA distribution system", 2001.

[20] N.R. Friedman "Distributed Energy Resources Interconnection Systems: Technology Review and Research needs." National Renewable Energy Laboratory, report SR-560-32459, September 2002.

[21] Khalid A. Nigim and Wei-Jen Lee "Micro Grid Integration Opportunities and Challenges", IEEE Xplore.

[22] A.D. Hawkes and M.A.Leach "Modeling high Level system design and unit commitment for a microgrid", Applied energy science digest Volume 86, JulyAugust 2008.

[23] O. A. Mohmmed, M. A. Nayeem and A. K. Kaviani "A Laboratory based Microgrid and Distributed Generation Infrastructure for studying Connectivity Issues to Operational Power Systems", IEEE Power Engineering Society General Meeting, July 2010.

[24] M.Chinchilla, S. Arnaltes and J.L. Rodriguez-Amenedo "Laboratory set-up for Wind Turbine Emulation", IEEE International Conference on Industrial Technology, 2004.

[25] Dale S. L. Dolan and P.W. Lehn "Real-Time Wind Turbine Emulator Suitable for Power Quality and Dynamic Control Studies", IPST, Canada, June 19-23, 2005.

[26] O.A.Mohammed, Luis Henao and A.A.Khan "Fuzzy Logic based Automatic Voltage Regulator System", IECON, Orlando, November 2008.

[27] AC Tech product library "ESVZARO ModBus Communications reference Manual". 
[28] AC Tech product library "ESVZARO ModBus Communications reference Manual".

[29] Stephen D.J.McArthur, Euan M. Davidson, Victoria M. Catterson, Aris L. Dimeas, Nikos D. Hatziargyriou, Fredinanda Ponci and Toshihisa Funabashi"Multi-Agent Systems for Power Engineering Applications - Part I : Concepts, Approaches, and Technical Challenges", IEEE Transactions on Power Systems, November 2007.

[30] Stephen D.J.McArthur, Euan M. Davidson, Victoria M. Catterson, Aris L. Dimeas, Nikos D. Hatziargyriou, Fredinanda Ponci and Toshihisa Funabashi"Multi-Agent Systems for Power Engineering Applications - Part II: Technologies, Standards, and Tools for Building Multi-Agent Systems", IEEE Transactions on Power Systems, November 2007.

[31] Fabio Bellifemine, Giovanni Caire, Tiziana Trucco, Giovanni Rimassa and Roland Mungenast "JADE Administrators Guide", Update 18-June-2007.

[32] Fabio Bellifemine, Giovanni Caire, Tiziana Trucco, Giovanni Rimassa and Roland Mungenast "JADE Programmers Guide", Update 18-June-2007. 


\section{APPENDIX}

\section{Appendix A - AS 440 AVR Implemented on Gen Station}

AS 440 AVR adaptable with Stamford generators, has been utilized for voltage regulation in the work. The Generator used is of brushless type, with the excitation available through a DC machine mounted on the shaft of the alternator. DC generator mounted is a self excited machine and residual voltage is used for voltage build up initially in the DC generator and then the field of the alternator. Figure 10.1 displays a picture of the AVR.

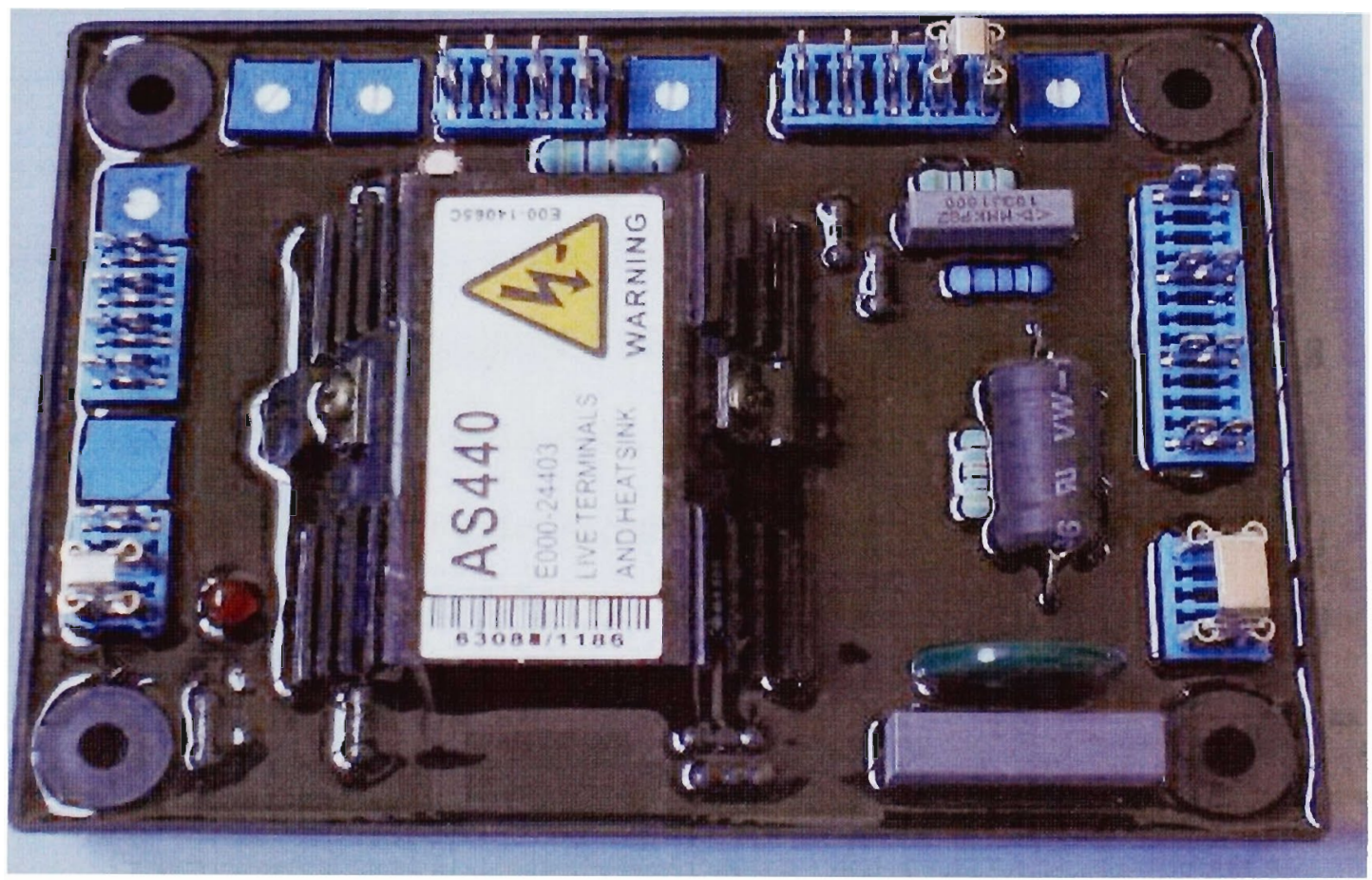

Figure A-1 AS 440 AVR

The AVR is linked with the main stator windings and the exciter field windings to provide closed loop control of the output voltage with load regulation of $+1-1.0 \%$. The 
AVR controls the power fed to the exciter field of the DC generator and hence the excitation field of the alternator eventually. It is also equipped with a frequency measuring circuit to monitor the generator output and provide under speed protection of the excitation system, by reducing the output voltage proportionally with speed below a pre-settable threshold. A manual adjustment is also present for setting of the under frequency roll of point, (UFRO). This also has a capability of 50 or $60 \mathrm{~Hz}$ operation selection.

A quadrature droop C.T. which provides a power factor dependent signal for the AVR is installed for parallel operation of generators. The DROOP adjustment is normally preset to give $5 \%$ voltage droop at full load zero power factor. An analog input (A1 A2) is provided to connect to a Power Factor Controller or other devices. It is designed to accept DC signals up to $+/-5$ Volts. The DC Signal applied to this input adds to the AVR sensing circuit. Al is connected to the AVR 0 volts. Positive on A2 increases excitation and negative on A2 decreases excitation. The trim control present on the AVR allows the user to adjust the sensitivity of the input, with trim fully anti-clockwise the externally applied signal has no effect. Figure 10.2 provides a brief summary of the actions which can be performed on the AVR. The AVR AS 440 manual can be referred to for more details. 


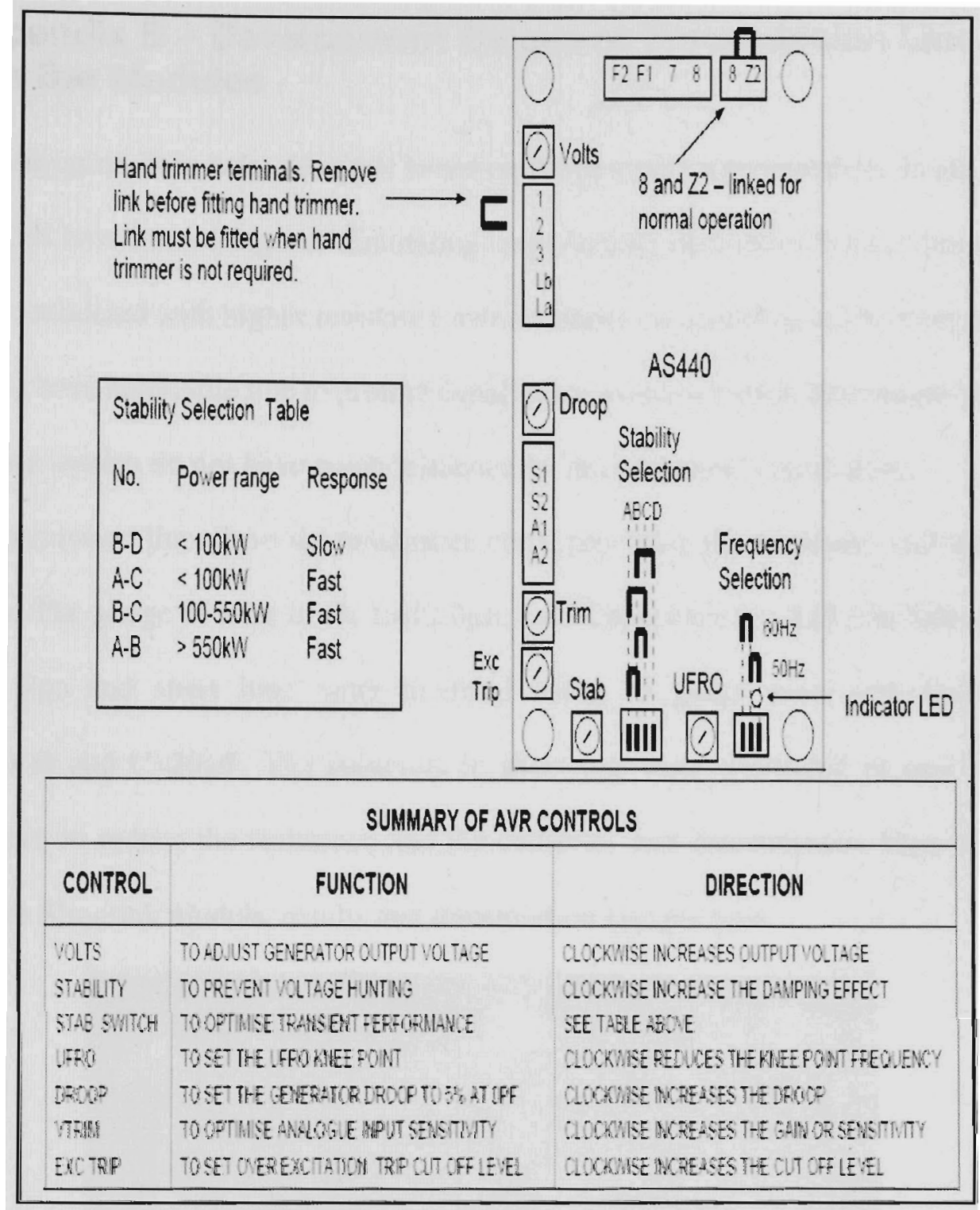

Figure A-2 Block Diagram of AVR 


\section{Appendix B - Development Details of Transmission Line and Bus Bar Modules}

The Transmission lines were designed based on the pi model representation. In all, three base models have been designed. Emulating long, medium and short distance lines, long lines were modeled with higher resistance and reactance compared to medium lines. The short lines have negligible line to ground capacitance as transmission lines employed by distribution system do not have much influence by line to ground capacitance.

Long transmission lines have six inductance coils, providing the resistance and inductive reactance. The gauge of wire is $18, \mathrm{~L}=12.0 \mu \mathrm{h}, \mathrm{L}-\mathrm{G}$ Capacitance $=2.2 \mu \mathrm{F}$ and $\mathrm{Rdc}=2.23 \Omega$. Medium line and short lines were modeled using 15 gauge wire with $\mathrm{L}=2.70 \mu \mathrm{H}$, $\mathrm{Rdc}=0.59 \Omega$ and $\mathrm{C}=20 \mu \mathrm{F}$. The inductors in short line were connected in parallel with pairs of two to reduce the resistance and the capacitor was disconnected. Figures below present the Simulink models, results and transmission line pictures.

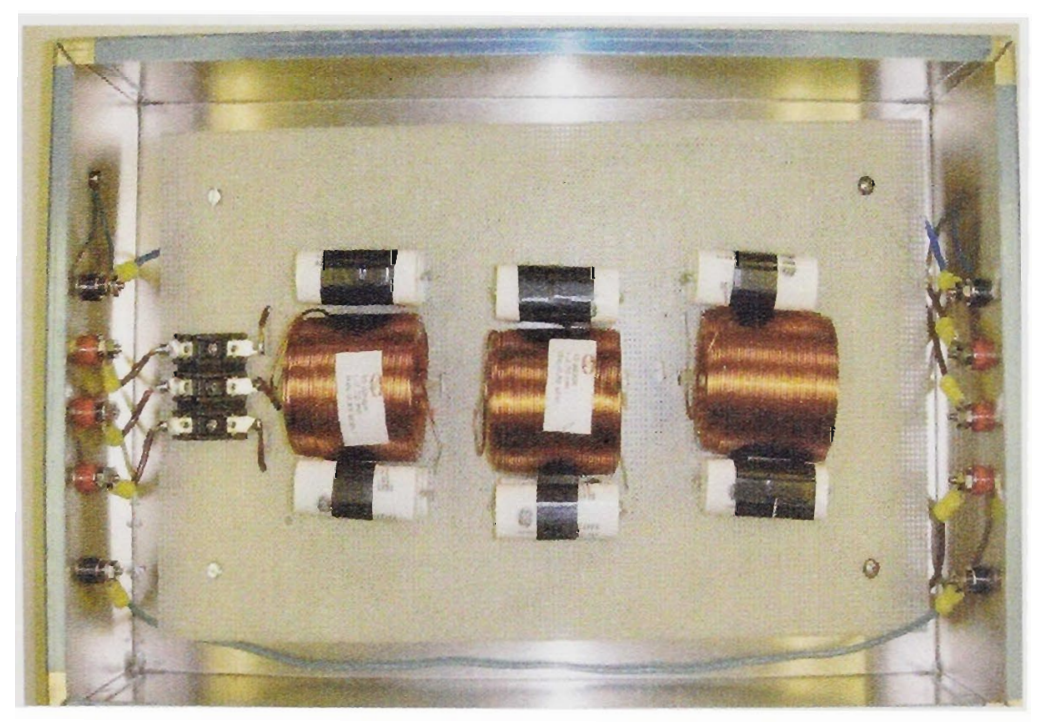

Figure B-1 Transmission Line Madel 


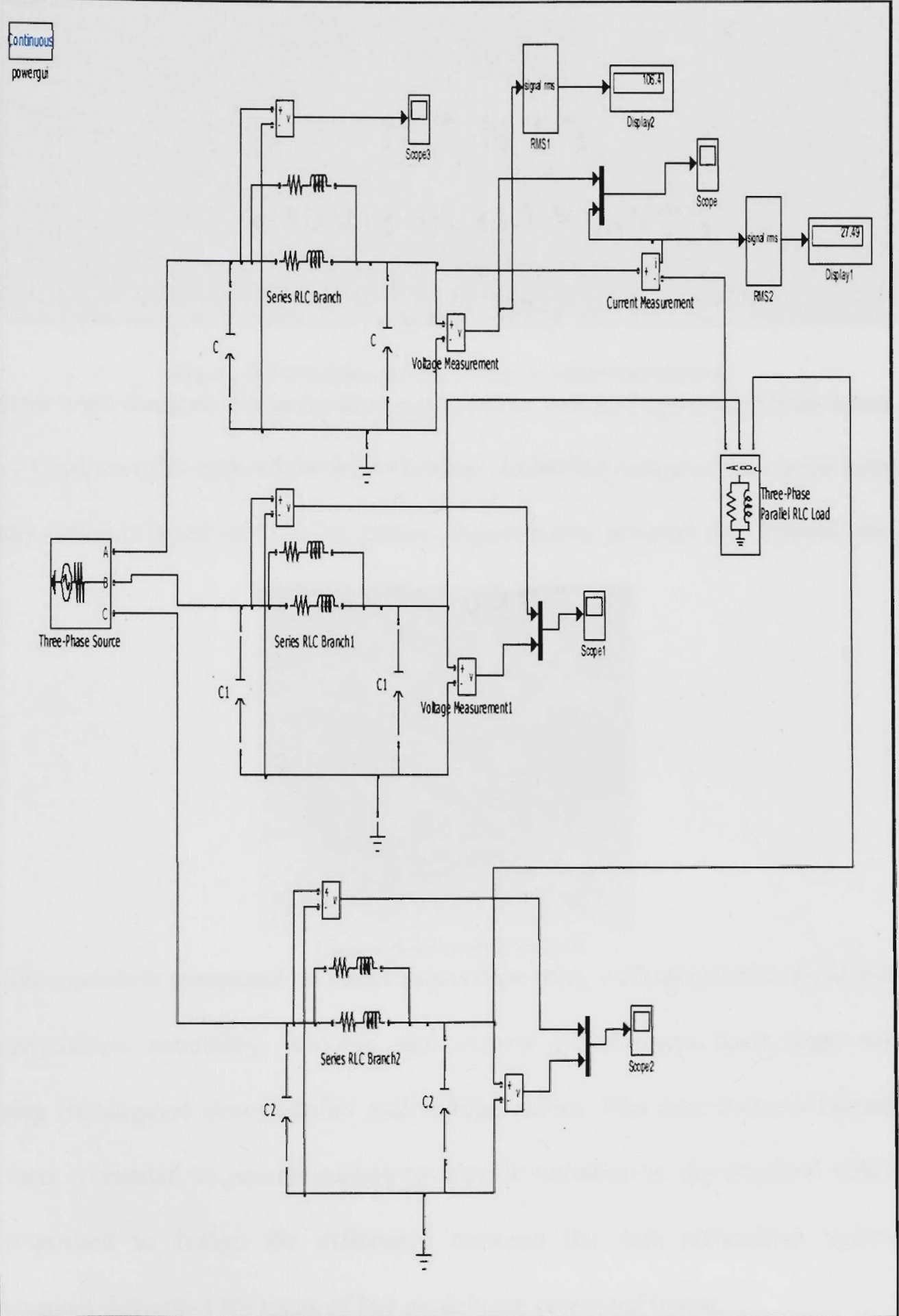

Figure B-2 Simulink model of Transmission Line 


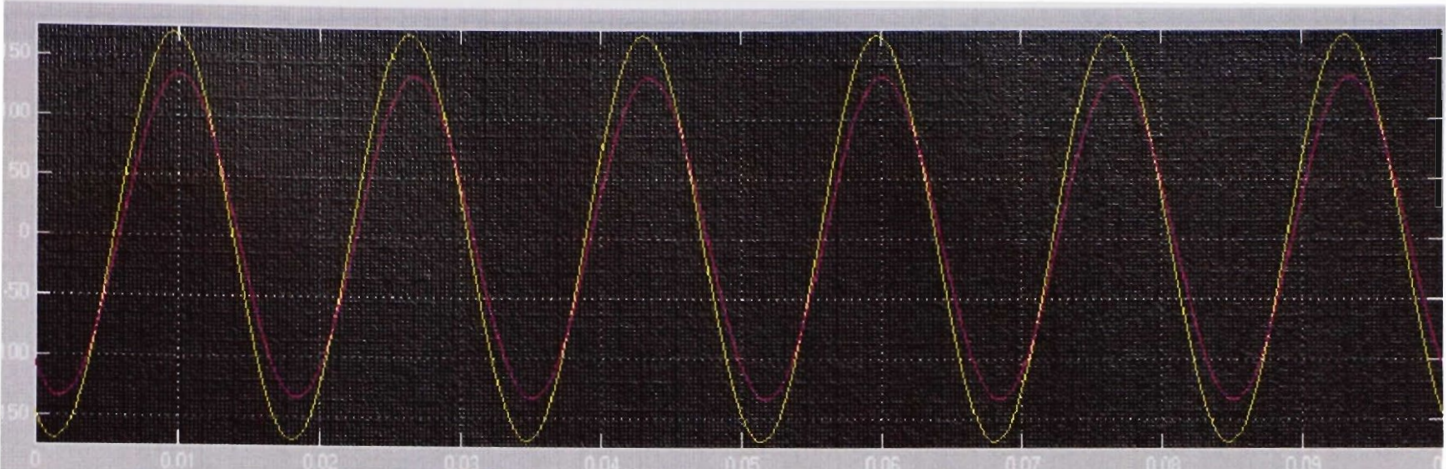

Figure B-3 Sending and Receiving terminal waveforms

The buses were designed for generator, synchronizer and load systems. All the buses

employ Crydom solid state relays for switching, simulating action of the circuit breaker, the relay action is based on SCADA system. Figure below presents the Crydom relay.

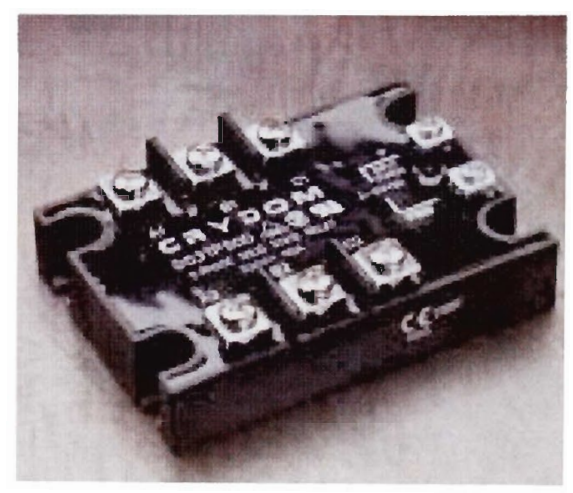

Figure B-4 Crydom Switch

Each bus module is connected on either sides of the relay with electrical connections witt data acquisition capability. Voltage and current transformers have been used fo: acquiring the stepped down current and voltage values. The measurement transformer: were later connected to potentiometers to provide variation in the acquired value. Thi: was important to bridge the difference between the data acquisition system an measurement devices. Two types of bus models are presented below. 


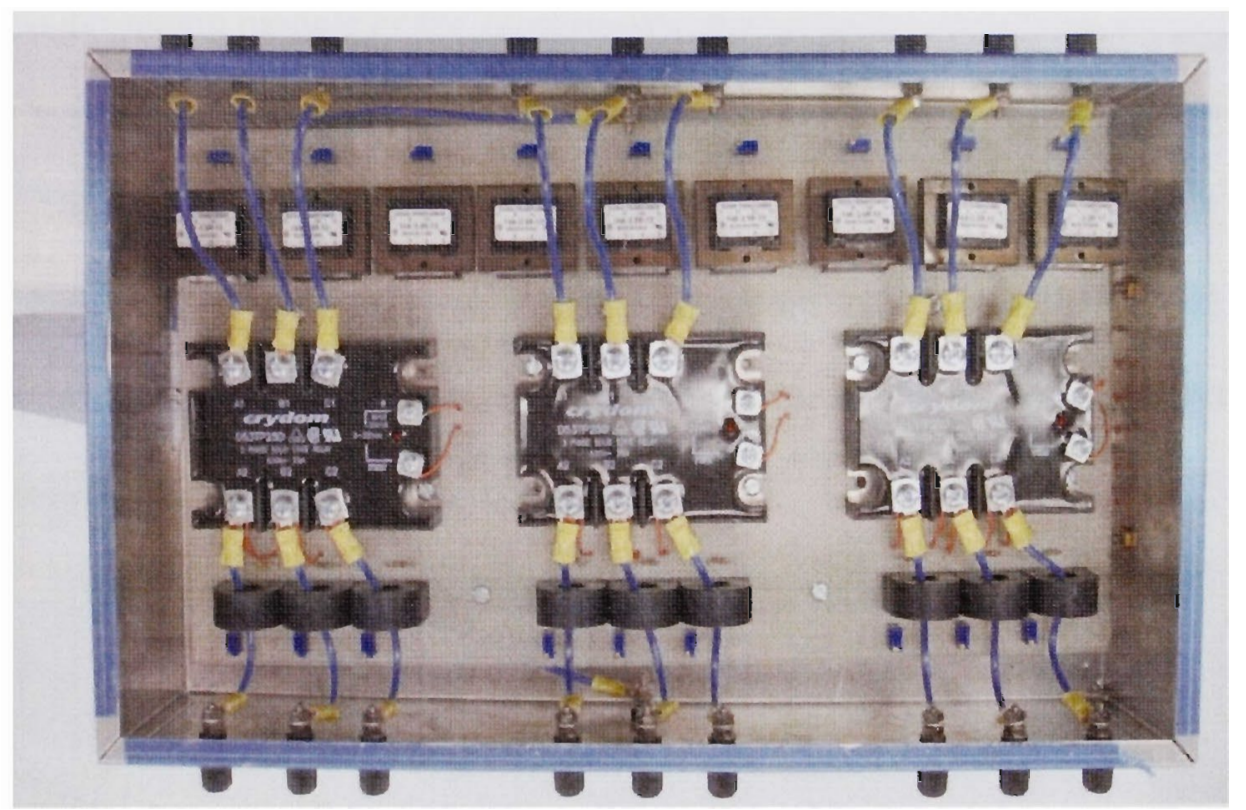

Figure B-5 Bus Model 3:3

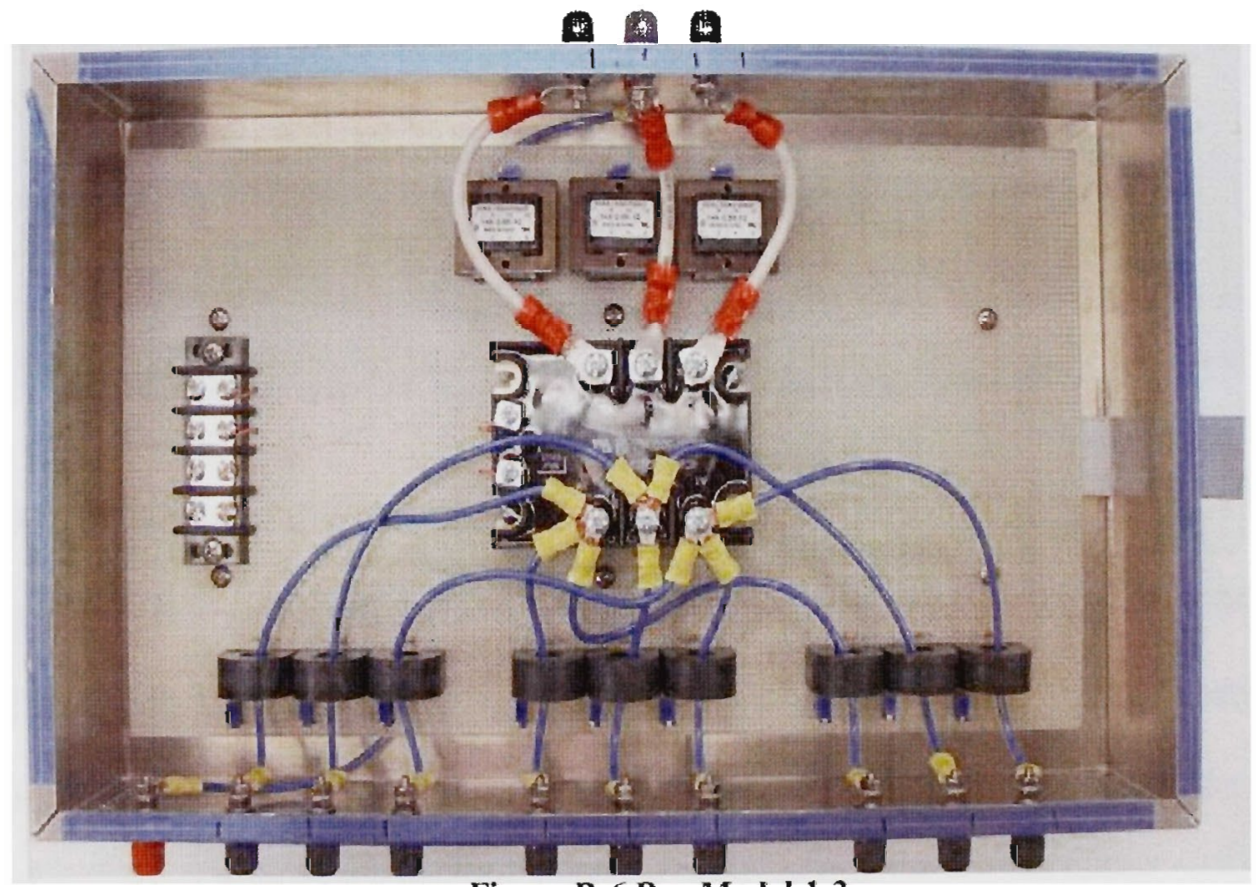

Figure B-6 Bus Model 1:3 
The synchronizing module or the synchronizer bus is another important component of the power system test bed. The custom design of this hardware is critical for the operation of the synchronizer module based on labview. The module has data acquisition capability on both sides of the relay to provide data to the software. The simulation and picture of the module are presented below. The figures also display the waveforms on the primary and the secondary of the measurement transformers.

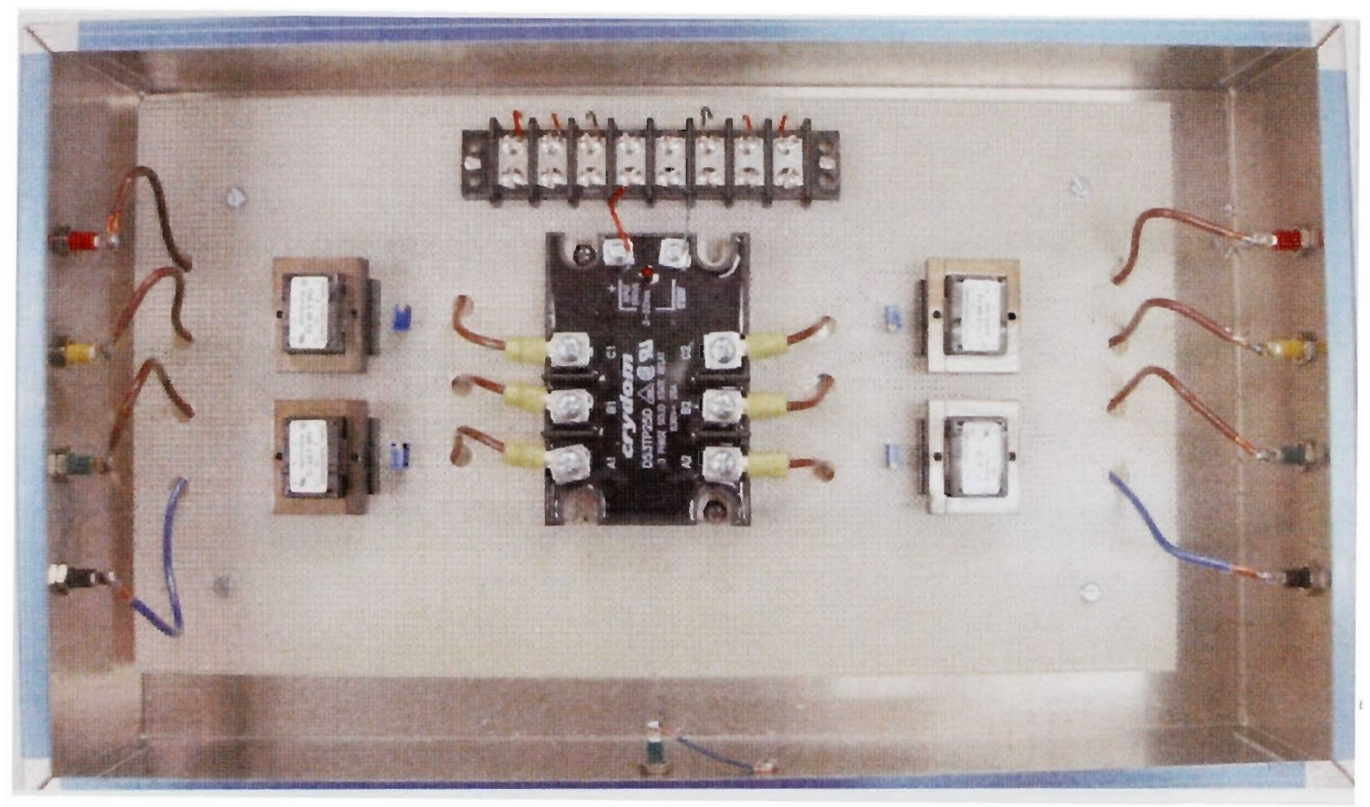

Figure B-7 Synchronizer Module

The data acquisition system comprises of Labview and dSPACE hardware modules. NI 6025E, NI 6071E and USB 6051M are the DAQ boards used for labview, among these $6071 \mathrm{E}$ has been used for critical applications on the power system for its faster response and reliability. $\mathrm{NI} 6071 \mathrm{E}$ is a100 pin control board. Its pin output is presented in the figure later. 


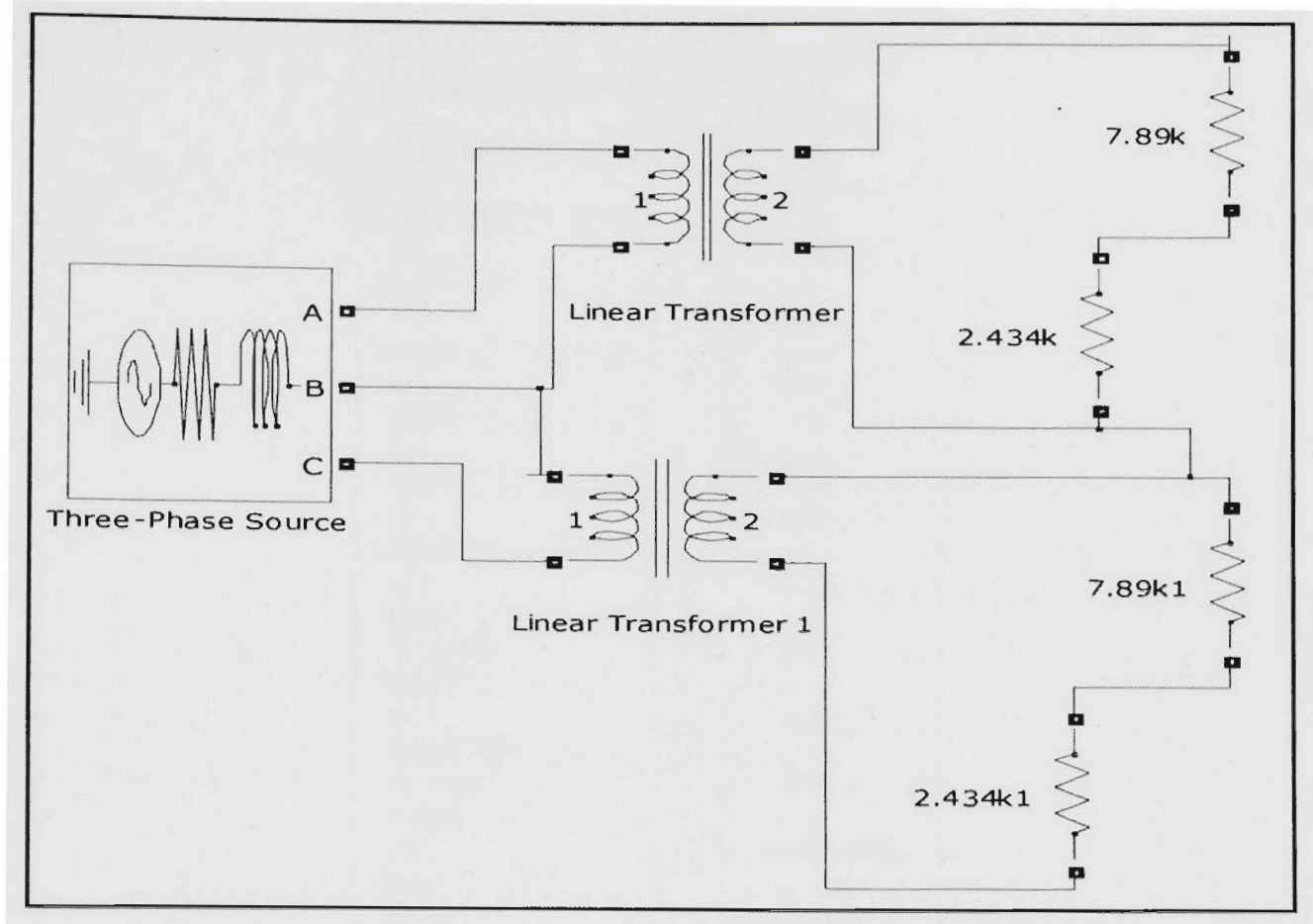

Figure B-8 Synchronizer module simulation

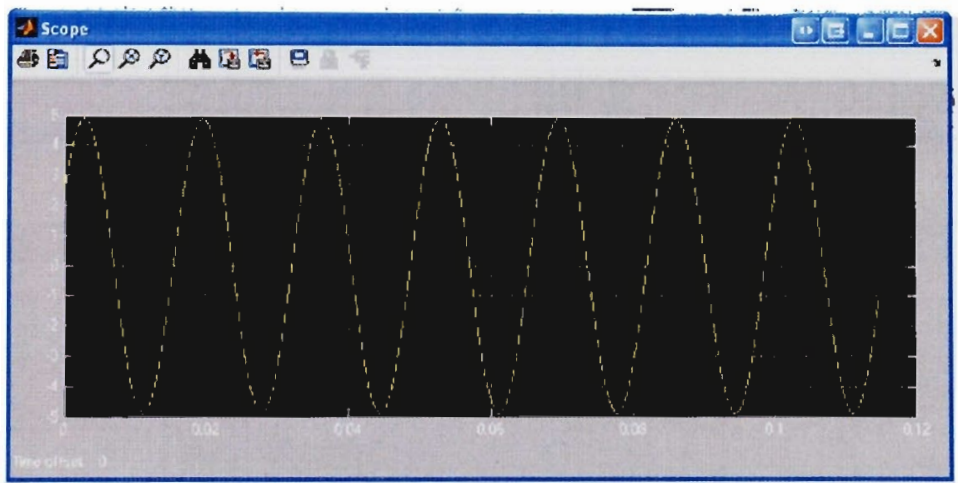

Figure B-9 Primary Voltage at Potential Transformer

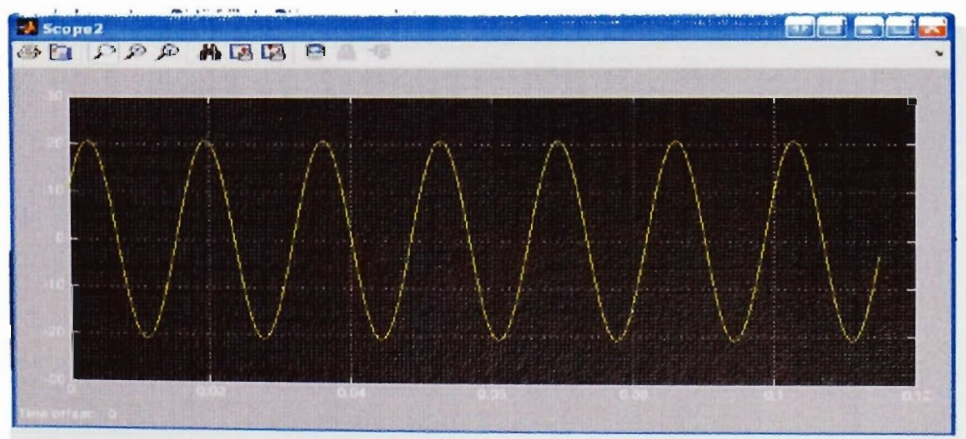

Figure B-10 Secondary Voltage at Potential Transformer 


\begin{tabular}{|c|c|c|c|}
\hline & & & \\
\hline AICND & ' & 51 & Al $1 \mathrm{~B}$ \\
\hline AI GND & $?$ & 32 & Al 244 \\
\hline AlO & 3 & 53 & A 17 \\
\hline$A \mid B$ & 4 & 54 & A' \\
\hline A 11 & 5 & 55 & Al 18 \\
\hline Al: & 6 & 5 & Al 96 \\
\hline Al: & $?$ & $5 \%$ & Al 19 \\
\hline Al 10 & 8 & 58 & Al $P Y$ \\
\hline Al 3 & 9 & 50 & AI 20 \\
\hline Al 11 & 10 & 60 & A) 28 \\
\hline Ald & 11 & 61 & Al 21 \\
\hline Al 12 & 12 & 62 & Al \\
\hline AI: & 13 & 63 & Al 22 \\
\hline Al 13 & 14 & 6.1 & A| 30 \\
\hline AIE & 15 & 65 & Al 23 \\
\hline Al 14 & 16 & 66 & Al 31 \\
\hline AI 7 & 17 & 67 & Al 32 \\
\hline Al 15 & 18 & 68 & 41,40 \\
\hline AI SLNSE & 19 & 80 & Al 33 \\
\hline AOO & 20 & 70 & $A \mid \$ 1$ \\
\hline AO 1 & 21 & 71 & At 34 \\
\hline AO EXT REF & 22 & 72 & A) 42 \\
\hline $\mathrm{AOGND}$ & 23 & 73 & Al 35 \\
\hline D GND & 24 & 74 & 41.83 \\
\hline Foo & 25 & 75 & AI SENSE 2 \\
\hline PO.4 & 26 & 70 & Al GND \\
\hline Po 1 & 27 & 77 & $A 136$ \\
\hline pos & 28 & 78 & S1 44 \\
\hline $\mathrm{PO} 2$ & 29 & 79 & Al 37 \\
\hline Po.6 & 30 & 90 & A1 4.5 \\
\hline$\rho_{0} 3$ & $3:$ & 81 & A. 38 \\
\hline PO? & 32 & 82 & $A \mid 46$ \\
\hline D GND & 23 & 83 & A1 39 \\
\hline $.5 V$ & 34 & 84 & AI \\
\hline .58 & 35 & 85 & Al 48 \\
\hline AIHOLD CORP & 35 & 86 & $\$ 150$ \\
\hline EXT STRICRE & 37 & (8) & Al 49 \\
\hline PFI OAA START TRQ & 321 & Hes & $A \mid S Y$ \\
\hline FFI I AD REF TAIA & 39 & 89 & 4150 \\
\hline FFI DAA CONV CIK & 40 & 50 & 4158 \\
\hline PFI SiOTA I SRe: & 41 & (11) & Al 51 \\
\hline PFI ACTP I GATE & 42 & A? & a) 5 \\
\hline CIA 1 OUT & 43 & H.S & A152 \\
\hline PFI S:AO SAMP GLK & $\$ 4$ & 44 & $A 1+0$ \\
\hline FFI GLO START TMIS & 45 & 95 & Al 53 \\
\hline PFI TIAI SAMP CLK & 45 & 96 & $A 161$ \\
\hline PFI BICTROSIRC & 47 & 97 & $.15 \times$ \\
\hline FFI 9,CTRO CATE & 48 & 98 & A) 62 \\
\hline CTR D OUT & 49 & 99 & Al 55 \\
\hline FRECOUT & 50 & 100 & Altis \\
\hline
\end{tabular}

Figure B-11 N16071E Pin Out 


\section{Appendix C - Education Demonstration Experiments}

\section{Synchronization of Alternators in Power Systems}

PURPOSE: To study the characteristics of Synchronization of Alternators to the Power System by a Digital Synchronizer DISCUSSION:

The concept of synchronizing to AC Machines is to ensure that they are operating in parallel and in tandem. In case of DC Machines it is easier to ensure it as the only parameter is voltage. In case of AC system the voltage variation is based on frequency, phase sequence and phase variation. This can be done manually by verifying the voltages in all three phases, verifying the frequency and phase sequence. Then the machines can be synchronized when the voltage difference between them is lowest, by using the dark light method. However, this concept can be explained in better manner by using the digital synchronization system used presently.

APPARATUS:

1. Labview Synchronizer VI

2. Hardware Synchronizer Module designed at Energy Systems Laboratory

3. Voltmeters for verification

\section{PROCEDURE:}

1. The labview synchronizer module provided at Energy Systems Laboratory should be connected to two AC Machines on either sides.

2. The Synchronizer Module is equipped with data acquisition blocks to provide information to the software blocks in labview.

3. The Digital Synchronizer VI should be activated on the computer.

4. The machines should be turned on both sides of the hardware module.

5. The waveforms on both sides of the module will be displayed as machine 1 and machine 2 on the VI.

6. The VI also displays frequency and voltage on both sides, it can be verified manually by using voltmeters.

7. Machines should be checked for identical voltage and frequency values.

8. At the point when both the waveforms overlap, the difference between the voltages is zero and the "SYNCHRONIZE" switch on the VI should be clicked tc synchronize the machines.

\section{QUESTIONS:}

1. What is the reason that you observe the waveforms to be moving on the plotter you see on Digital Synchronizer VI?

2. What will happen when you synchronize when the parameters are not identical?

3. What is Hunting? 

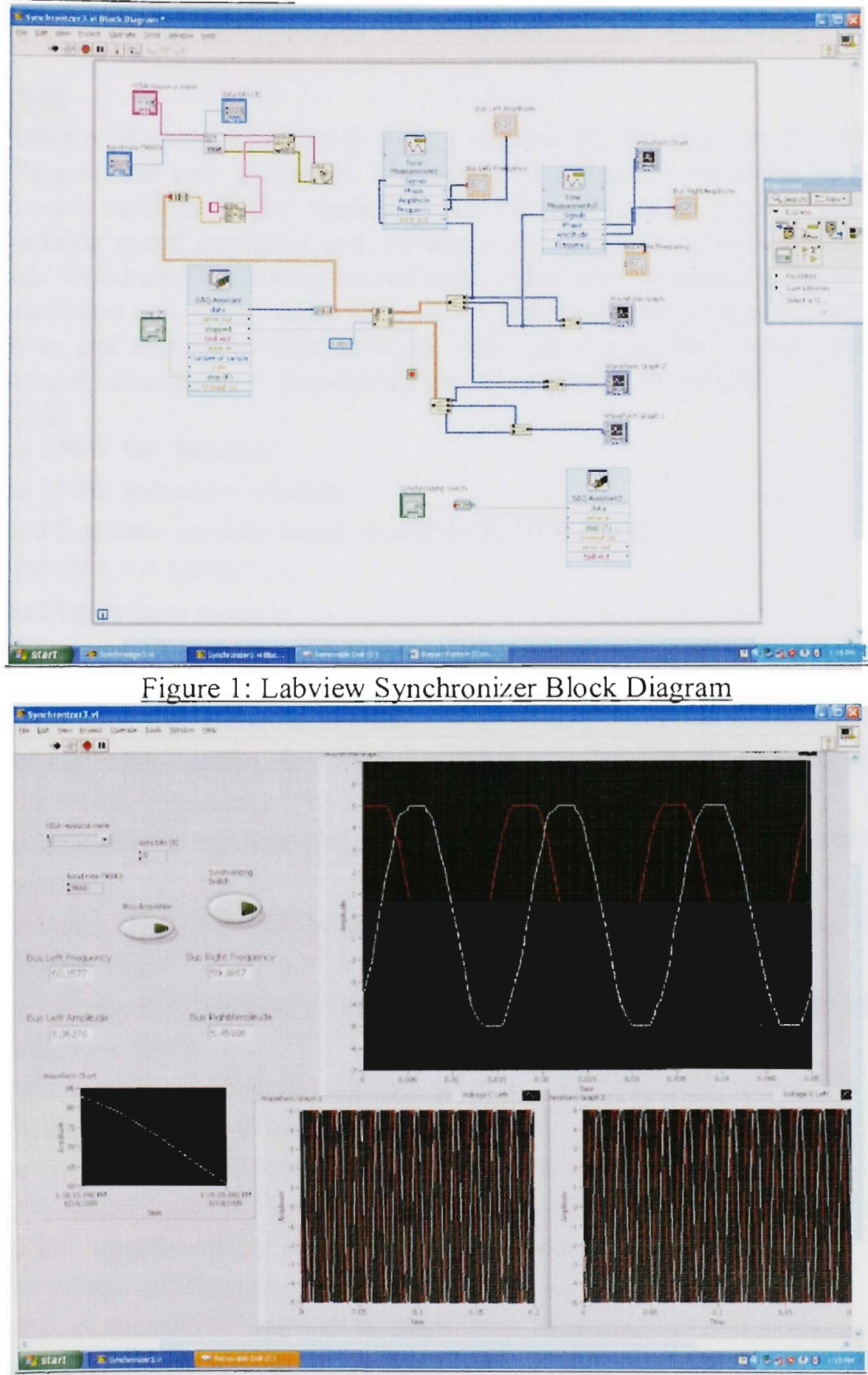

Figure 2: Labview Synchronizer GUI 


\section{Operation of Induction Machine based Wind Generator}

PURPOSE: To study the characteristics of Induction Generator based Wind Turbine Generator DISCUSSION:

The utilization of non-conventional energy sources for electrical energy has thrown many challenges and new prospects. Utilization of Induction machines as generators coupled to wind turbines is also applied. Induction machines are used as generators as they are variable speed machines and are also very robust. Synchronous machines are also used and they have their own pros and cons. Induction machines do not have explicit field for excitation and hence when operated as generators they require reactive power for starting up and also for voltage build up. They can acquire this reactive power either through charged capacitance or through the grid if connected to the grid.

APPARATUS:

4. One $250 \mathrm{~W}$ DC Machine

5. One $250 \mathrm{~W}$ Induction Machine

6. Wind Emulator module based on dSPACE-1104 board

7. dSPACE 1104 MODULE

8. One Capacitance module

\section{PROCEDURE:}

9. The DC Machine is used as a prime mover to emulate wind turbine.

10. The real time model for wind emulator can be run for providing random variations in the speed of the DC Machine.

11. The capacitance module should be charged before connecting to the induction generator.

12. The value of voltage and the starting waveform should be recorded as soon as the generator starts.

13. The voltage and frequency value of the induction machine should be recorded a1 steady state mode.

14. Apply a load of 150 watts on the generator in steps and record the values 0 voltage, current and frequency.

15. The waveforms should also be recorded for the above steps.

16. In the second part the generator should be connected to the grid before starting and the capacitance box should be disconnected.

17. The voltage and frequency values should be recorded at start up.

18. The load should be applied in steps to a maximum of $150 \mathrm{watts}$ and voltage current and frequency should be recorded.

\section{QUESTIONS:}

4. What is the reason that induction machine needs capacitance to start up, can $i$ work with inductance? 
5. What is the difference between frequency and voltage on loading when capacitance box is connected?

6. What is the reason for frequency variation and what is the reason for voltage variations?

7. Why is there a difference between start up voltage when capacitance is connected and when grid is connected?
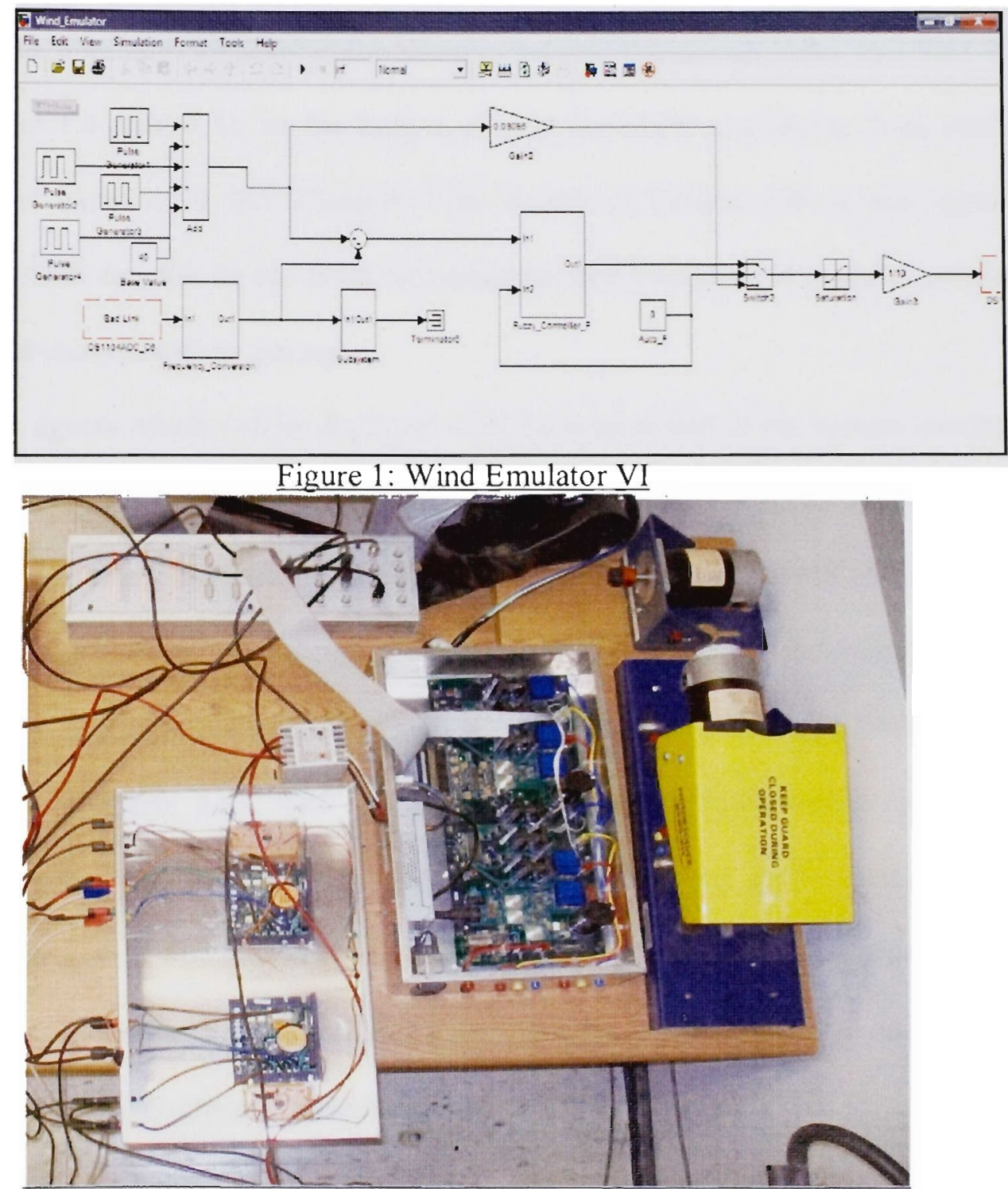

Figure 2: Power Electronic Module Setup 


\section{Appendix C - Simulation Code of an Agent System in Eclipse using JADE for implementation on Test Bed}

JADE is Java Agent Development Framework, it is based on the Java language and JADE provides the API (Application Programming Interface) for developing agent based systems. The minimum software requirement to execute the system is the Java Run Time Environment 1.4 (JRE 1.4). As the Eclipse plug in for JADE eJADE has been used for development based on a GUI (Graphic User Interface), Eclipse IDE is also required. Although JADE can still be run from the command line interface but it makes programs difficult and complicated to manage.

The default agents which will be deployed with the deployment of the system are (Agent Management System) AMS and (Directory Facilitator) DF. The AMS is an important component and every agent on the platform registers itself with the AMS and is provided a unique Agent Identifier for management purposes. The directory facilitator is another service agent, basically every agents which intends to communicate with other agents on the platform, registers itself with the directory facilitator. The directory facilitator is similar to yellow pages service providing agent identifiers and agent location to other agents on the system which wish to communicate. JADE Administrators guide and JADE programmers guide can be referred to for more details. The code for the simple energy management agent program is presented below.

\section{Buyer Agent}

import jade.domain.FIPAException;

import jade.domain.FIPAAgentManagement.DFAgentDescription; import jade.domain.FIPAAgentManagement.ServiceDescription;

public class PowerBuyerAgent extends Agent \{ 
$/ /$ The name of the microgrid

private String targetBookTitle;

// The list of known seller agents

private AID[] sellerAgents;

// Put agent initializations here

protected void setup() \{

$/ /$ Printout a welcome message

ready.");

System.out.println("Hallo! Buyer-agent "+getAID().getName()+" is

// Get the title of the book to buy as a start-up argument

Object [] args = getArguments();

if (args != null \&\& args. length $>0)\{$

targetMicrogridTitle $=($ String $)$ args $[0] ;$

System.out.println("Target Microgrid is "+targetMicrogridTitle);

// Add a TickerBehaviour that schedules a request to seller agents addBehaviour(new TickerBehaviour(this, 60000) \{

protected void onTick() \{

System.out.println("Trying to buy

"+targetMicrogridTitle);

// Update the list of seller agents

DFAgentDescription template $=$ new DFAgentDescription();

ServiceDescription sd = new ServiceDescription();

sd.set Type("Power-selling");

template.addServices(sd);

try \{

DFAgentDescription[] result = DFService. $\operatorname{search}($ myAgent, template $)$;

System.out.println("Found the following seller agents:");

sellerAgents = new AID $[$ result. length];

for (int $\mathrm{i}=0 ; \mathrm{i}<$ result. length; $++\mathrm{i}$ ) \{

sellerAgents[i] $=\operatorname{result}[i] \cdot \operatorname{get} \operatorname{Name}()$;

System.out.println(sellerAgents[i].getName());

\}

\}

catch (FIPAException fe) \{

fe.printStackTrace();

\}

$/ /$ Perform the request

myAgent.addBehaviour(new RequestPerformer());

\});

\} 


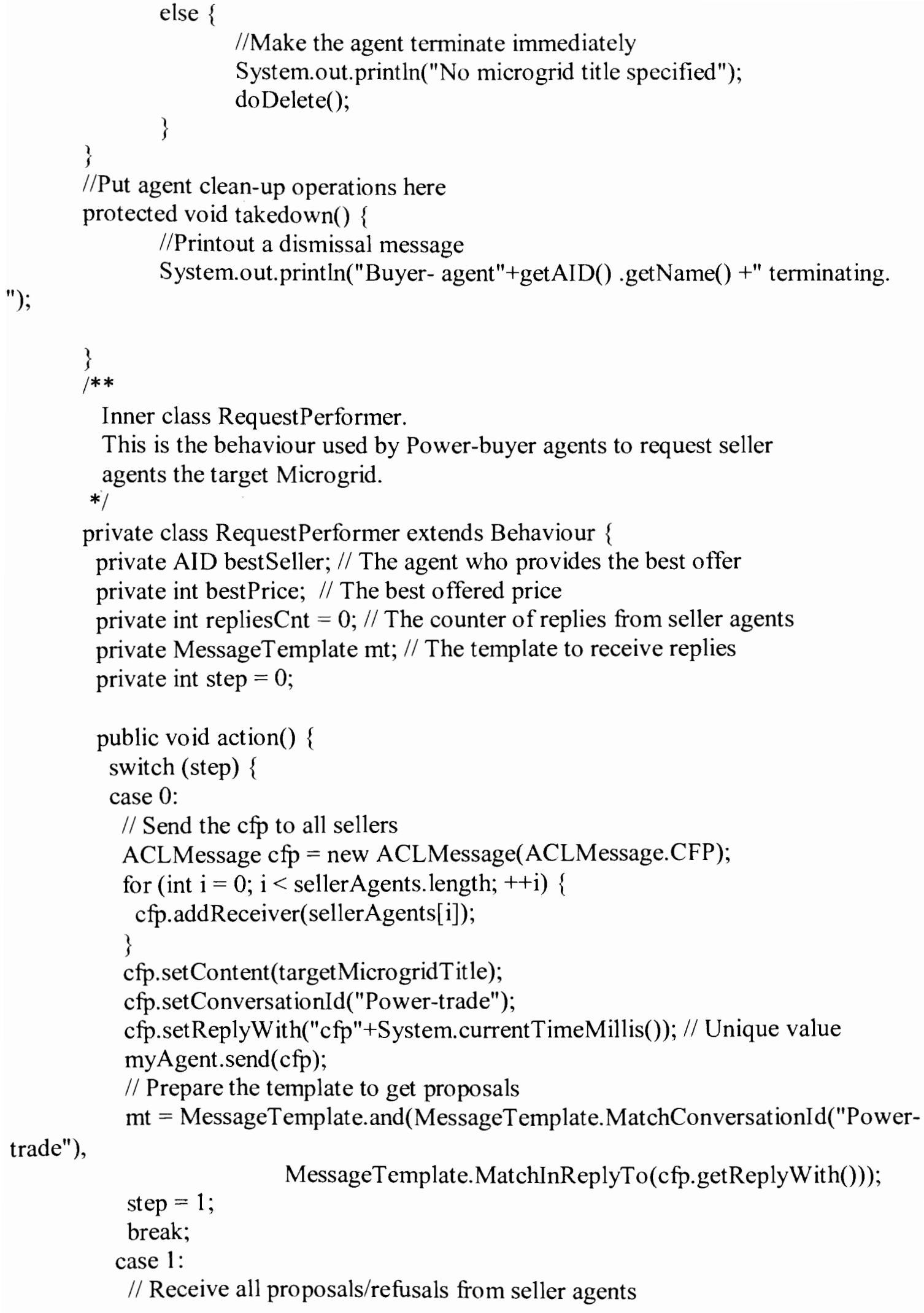

// Receive all proposals/refusals from seller agents 
ACLMessage reply $=$ myAgent $\cdot$ receive $(m t) ;$

if (reply != null) \{

// Reply received

if (reply.getPerformative ()$==$ ACLMessage.PROPOSE)

// This is an offer

int price $=$ Integer . parseInt $($ reply.getContent ()$)$;

if $($ bestSeller $==$ null $\|$ price $<$ bestPrice) \{

// This is the best offer at present

bestPrice $=$ price;

bestSeller $=$ reply.getSender();

\}

repliesCnt++;

if (repliesCnt $>=$ sellerAgents. length) \{

$/ /$ We received all replies

step $=2$;

\}

\}

else \{

block();

\}

break;

case 2:

// Send the purchase order to the seller that provided the best offer

ACLMessage order $=$ new

ACLMessage(ACLMessage.ACCEPT_PROPOSAL);

order.addReceiver(bestSeller);

order.setContent(targetBookTitle);

order.setConversationId("Power-trade");

order.setReplyWith("order"+System.currentTimeMillis());

myAgent.send(order);

// Prepare the template to get the purchase order reply

$\mathrm{mt}=$ MessageTemplate. and(MessageTemplate. MatchConversationId("Power-

trade"),

step $=3$;

MessageTemplate.MatchInReplyTo(order.getReplyWith()));

break;

case 3:

// Receive the purchase order reply

reply = myAgent .receive $(\mathrm{mt})$;

if (reply != null) \{

// Purchase order reply received

if $($ reply.getPerformative ()$==$ ACLMessage.INFORM)

// Purchase successful. We can terminate 
System.out.print $\ln (\operatorname{targetMicrogrid}$ Title+" successfully purchased from agent "+reply.getSender().getName());

System.out.println("Price $=$ "+bestPrice);

myAgent.doDelete();

\}

else \{

System.out.println("Attempt failed: requested Microgrid is unwilling."); \}

step $=4$

\}

else \{

block ();

\}

break;

\}

\}

public boolean done() \{

if $($ step $==2 \& \&$ bestSeller $==$ null)

System.out.println("Attempt failed: "+targetMicrogridTitle+" not

available for sale");

i

return $(($ step $==2 \& \&$ bestSeller $==$ null $) \|$ step $==4)$;

\}

\}

\} // End of inner class RequestPerformer

Seller Agent

import jade.core.Agent;

import jade.core.behaviours. ${ }^{*}$;

import jade.lang.acl. ACLMessage;

import jade.lang.acl.MessageTemplate;

import jade.domain.DFService;

import jade.domain.FIPAException;

import jade.domain. FIPAAgentManagement.DFAgentDescription;

import jade.domain.FIPAAgentManagement.ServiceDescription;

import java.util.*;

/** Inner class offerRequestsServer.

* This is the behaviour used by Power-Seller agents to serve incoming requests for offer

* from buyer agents. 
* If the requested Power is in the local catalogue the seller agent replies

* with a PROPOSE message specifying the price. otherwise a REFUSE message is sent back.

*

*@author M.A.Nayeem

*

*/

public class PowerSellerAgent extends Agent \{

// The catalog of Power for sale (maps the title of a Power to its price)

private Hashtable catalogue;

// The GUI by means of which the user can add Power in the Catalogue private PowerSellerGui myGui;

// Put Agent initializations here protected void setup() \{

// Create the catalogue

catalogue $=$ new Hashtable () ;

// Create and show the GUI

myGui = new PowerSellerGui(this);

myGui.show();

// Register the Power-selling service in the yellow pages

DFAgentDescription dfd = new DFAgentDescription();

dfd.setName(getAID());

ServiceDescription sd = new ServiceDescription();

sd.setType("Power-Trading");

sd.setName("JADE-Power-trading");

dfd.addServices(sd);

try \{

DFService.register(this, $\mathrm{dfd})$;

\}

catch (FIPAException fe) \{

fe.printStackTrace(); \}

// Add the behaviour serving queries from buyer agents addBehaviour(new offerRequestsServer());

// Add the behaviour serving purchase orders from buyer agents addBehaviour(new PurchaseOrdersServer());

\}

// Put agent clean-up operations here 


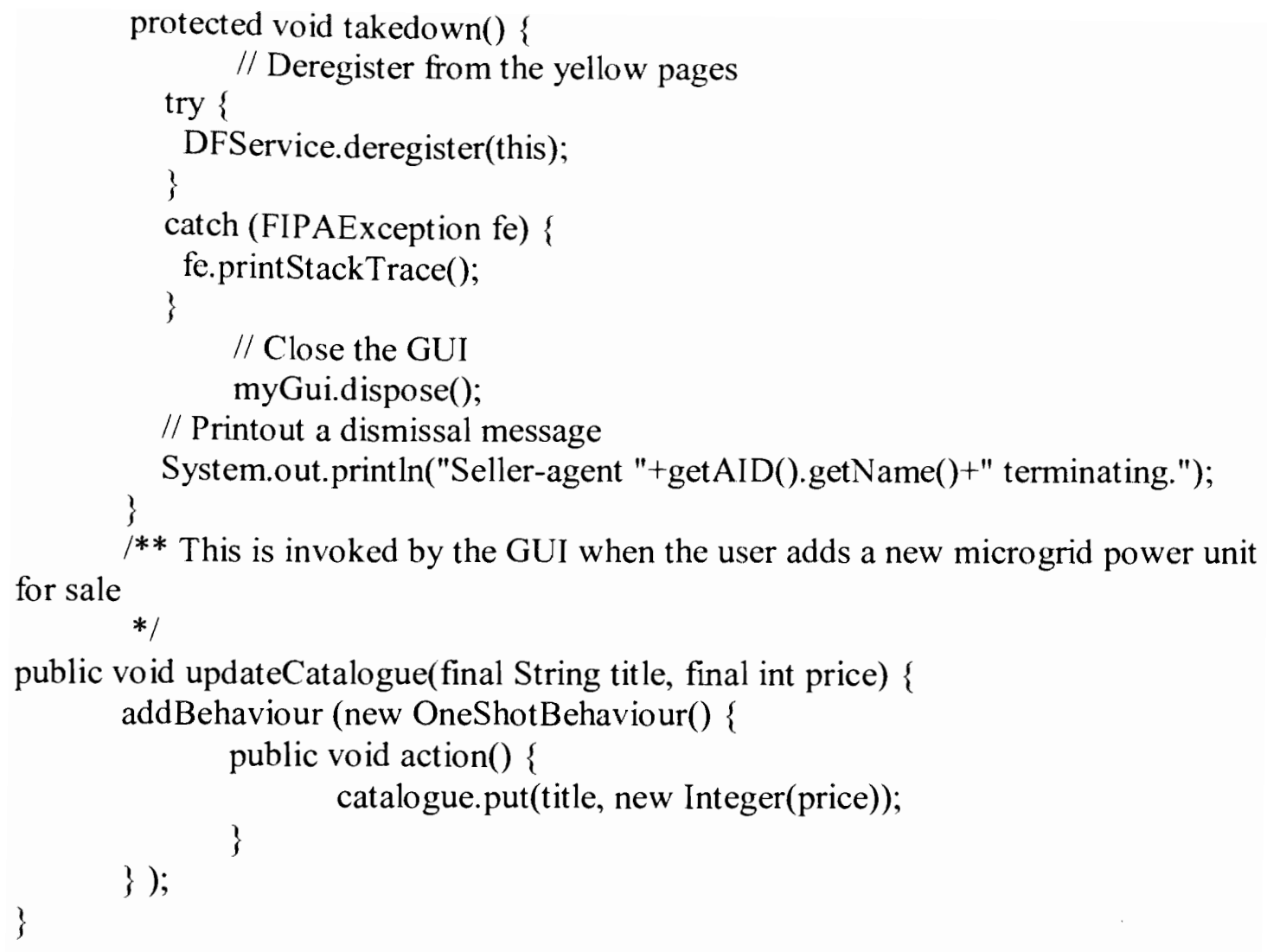

private class offerRequestsServer extends CyclicBehaviour \{ public void action () \{

ACLMessage msg = myAgent.receive ();

if (msg != null) \{

// Message received. Process it

String title $=$ msg.getContent () ;

ACLMessage reply $=$ msg.createReply () ;

Integer price $=($ Integer $)$ catalogue.get(title $)$;

if (price !=null)

the price

// The requested microgrid is ready for trade. Reply with reply.setPerformative(ACLMessage.PROPOSE); reply.setContent(String.valueOf(price.intValue()));

; else \{

// The requested microgrid not ready for trade. reply.setPerformative(ACLMessage.REFUSE); reply.setContent("not-available"); 


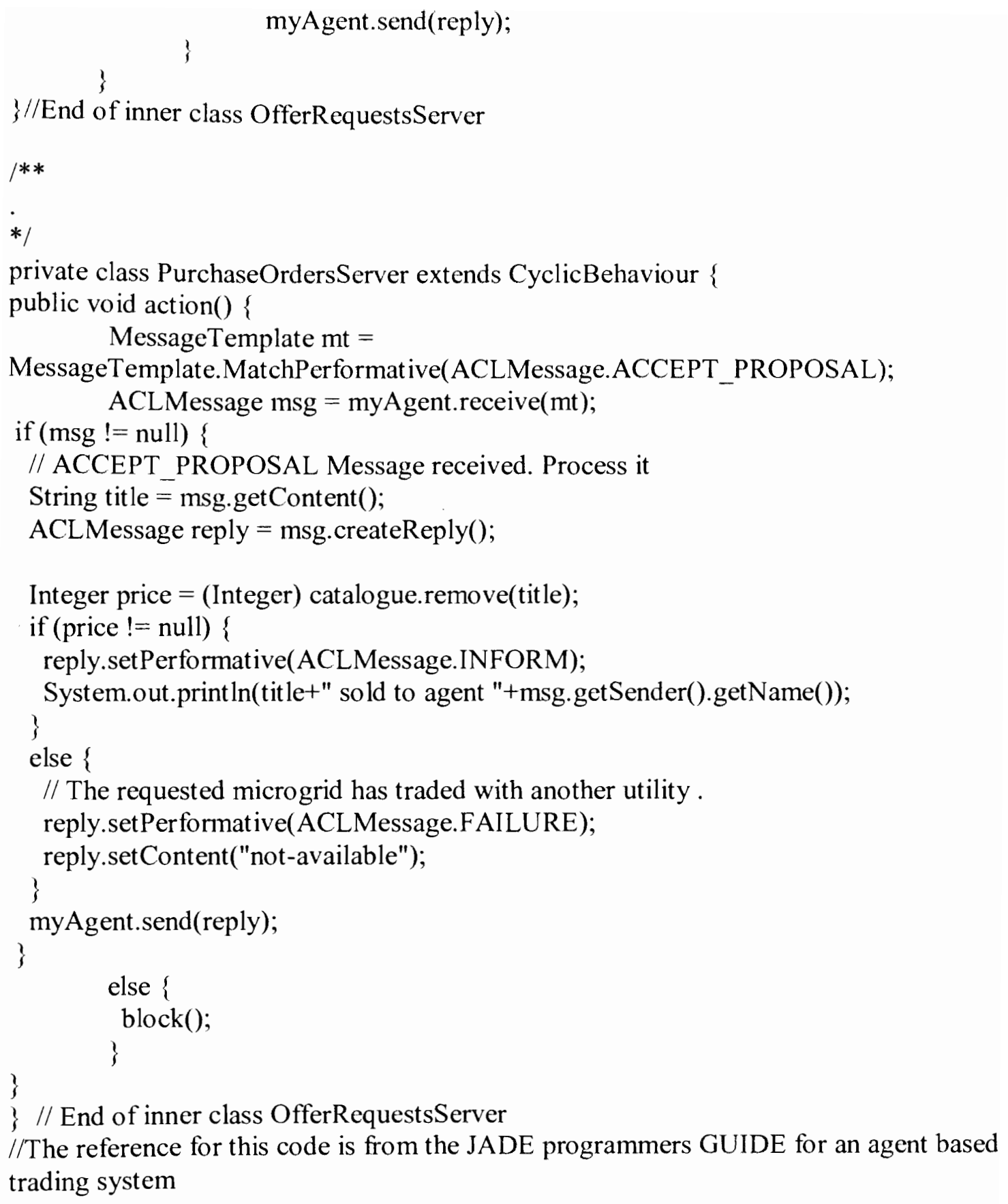




\section{Appendix D - User Manual for Test Bed Operation}

The sequences of steps to be followed to start the power system test bed and operate it are presented as follows. Firstly the power system is to be started, it requires starting the generating stations, the prime movers required for the experiment can be selected and mechanically coupled to the alternator using belt, from different machines mounted on the rack. The block diagram representing the power system skeleton is presented in figure 10.3 .

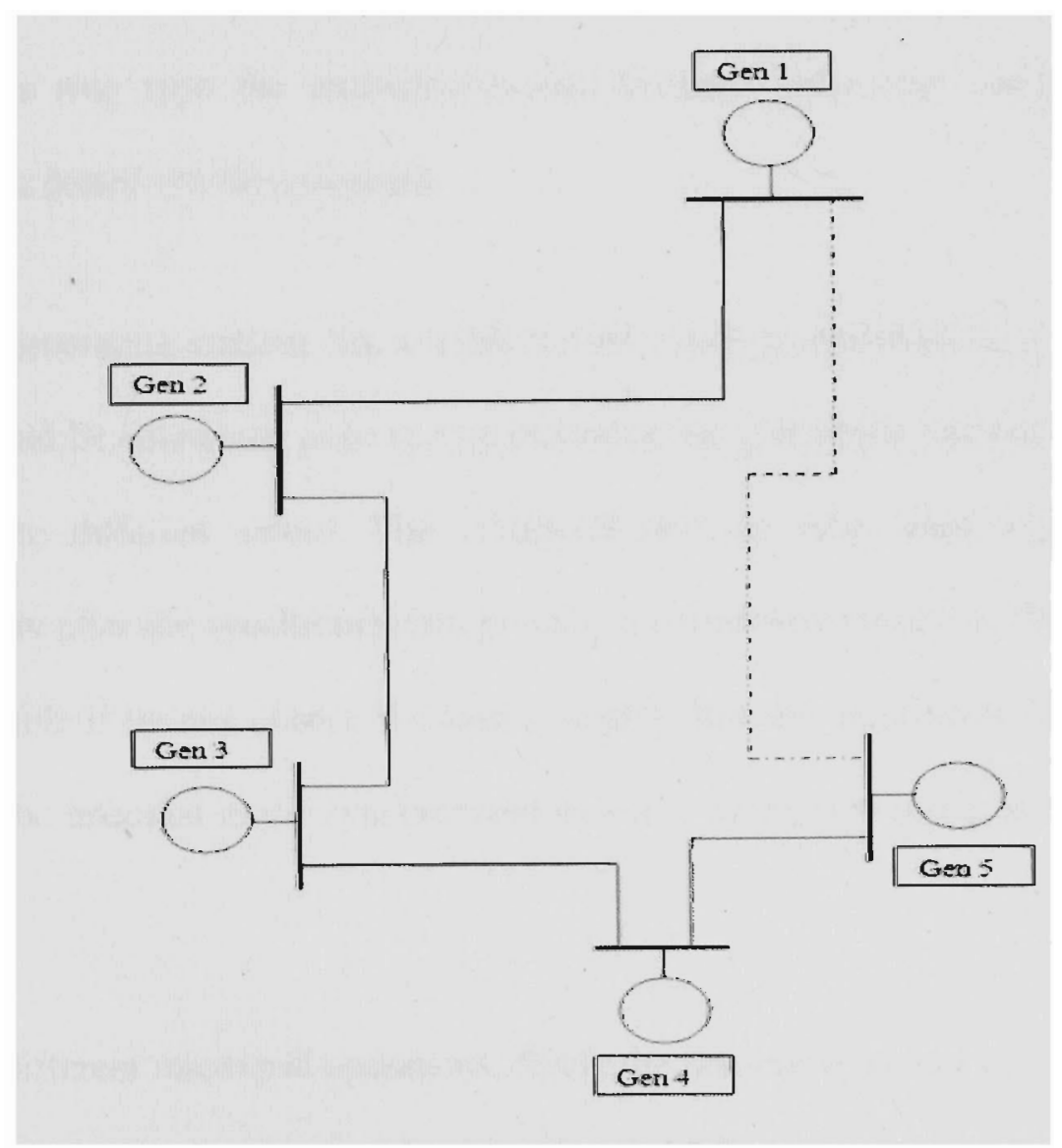

Figure D-1 Generating Stations Connection Skeleton 
The continuous lines connecting the generating stations in the block diagram are equipped with synchronizing modules and they are to be synchronized initially. The generating stations can be synchronized in any order as long as one machine is synchronized at a given time instance. The order which was followed during the experimental work followed a sequence of synchronizing generating station number 5 and 4 initially. Then generating station 3 was synchronized with the previous set. Another set with generating stations 1 and 2 was synchronized separately and then these two sets were synchronized to form a complete power system. To convert this power system into a ring type the transmission line between generating stations 5 and 1 displayed by a dotted can be connected.

Each of the generating stations has a internal load which is emulated by a 3 phase load box, this should be connected prior to synchronizing the generating stations and can also be adjusted to different values. The microgrid and the other load areas are to be connected only after the synchronization process is completed, otherwise the system will become unstable if by any chance the load is higher than the generation. The microgrid initially will be operated in the synchronized power consumption mode, acting as a load center.

To perform different microgrid operations, firstly the non-conventional sources should be operated. PV array can be turned on by operating the DC power supply. The wind turbine emulator is started by executing the real time simulink model for wind speed emulator, then the rectifier module is activated from Labview. The combustion generator or the 
stand alone back up inverter should be turned on to provide the voltage and frequency reference to the microgrid for islanded operation. The microgrid management system VI needs to be executed for the Labview environment and DAQ devices to function according to the design. eJADE environment in Eclipse IDE on the computer systems needs to be activated and deployed in order to create and test any multi agents or to program any agent system.

After performing the above mentioned states, the test bed starts functioning completely, any experiments can be performed on the test bed as long as they do not disturb the stability of the system. 


\section{Appendix E-m-File used for evaluating the dSPACE logged data from Experiments}

$t=x . X$. Data; \% time for each experiment $=20 \mathrm{~s}$

$I d c=x . Y(1,1) \cdot$ Data;

$\mathrm{Vdc}=\mathrm{x} . \mathrm{Y}(1,2)$. Data;

$\mathrm{IL}=\mathrm{x} . \mathrm{Y}(1,3)$. Data;

$\operatorname{Ig}=x . Y(1,4) \cdot$ Data;

Iinv $=x . Y(1,5) \cdot$ Data;

$\mathrm{IL}=\mathrm{x} . \mathrm{Y}(1,6)$. Data;

$\mathrm{Vg}=\mathrm{x} . \mathrm{Y}(1,7)$. Data;

Vinv $=x . Y(1,8) \cdot$ Data;

$\% \%$ Graphs

$\%$

figure(1)

subplot $(2,1,1)$

plot $(\mathrm{t}, \mathrm{Ig})$

xlabel('time')

ylabel('Generator Current')

subplot $(2,1,2)$

plot $(\mathrm{t}, \mathrm{Vg})$

xlabel('time')

ylabel('Generator Voltage')

$\%$

figure(2)

subplot $(2,1,2)$

plot(t, VL)

xlabel('time')

ylabel('Load Voltage')

$\operatorname{subplot}(2,1,1)$

$\operatorname{plot}(\mathrm{t}, \mathrm{IL})$

xlabel('time')

ylabel('Load Current')

$\%$

figure(3)

$\operatorname{subplot}(2,1,2)$

plot (t, Vinv)

xlabel('time')

ylabel('Inverter Voltage') 


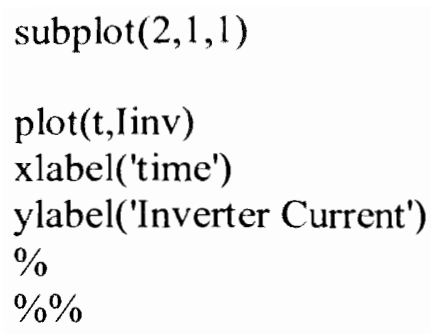

